\title{
Enhanced nonlinear optical responses of materials: Composite effects
}

\author{
J.P. Huang ${ }^{\mathrm{a}, *}$, K.W. Yu ${ }^{\mathrm{b}}$ \\ ${ }^{a}$ Surface Physics Laboratory (National key laboratory) and Department of Physics, Fudan University, Shanghai 200433, China \\ ${ }^{\mathrm{b}}$ Department of Physics and Institute of Theoretical Physics, The Chinese University of Hong Kong, Shatin, New Territories, Hong Kong
}

Accepted 19 May 2006

Available online 7 July 2006

editor: A.A. Maradudin

\begin{abstract}
We review recent theoretical progress in understanding physical processes of composite effects on enhanced third-order nonlinear optical responses of various kinds of the recently-proposed nonlinear optical materials, namely, colloidal nanocrystals with inhomogeneous metallodielectric particles or a graded-index host, metallic films with inhomogeneous microstructures adjusted by ion doping or temperature gradient, composites with compositional gradation or graded particles, and magneto-controlled ferrofluidbased nonlinear optical materials.
\end{abstract}

(C) 2006 Published by Elsevier B.V.

PACS: 42.70.-a; 77.84.Lf; 42.65.An; 42.65.-k

Keywords: Enhanced nonlinear optical responses; Composite effects; Nonlinear optical materials; Graded composite materials

\section{Contents}

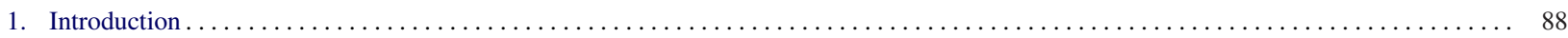

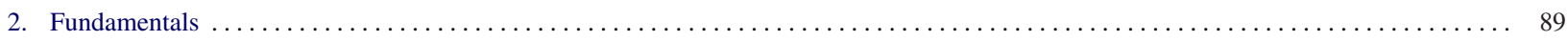

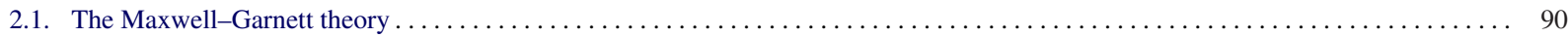

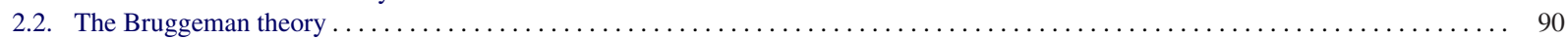

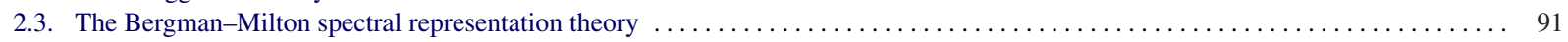

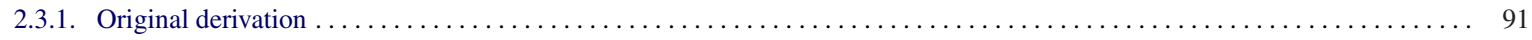

2.3.2. An easy-to-understand illustration of the Bergman-Milton spectral representation $\ldots \ldots \ldots \ldots \ldots \ldots$

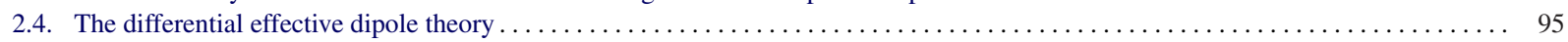

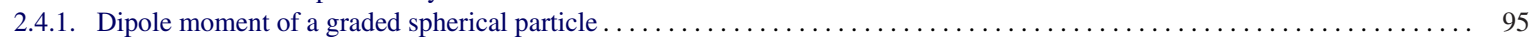

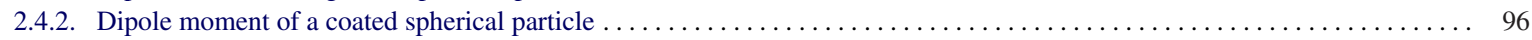

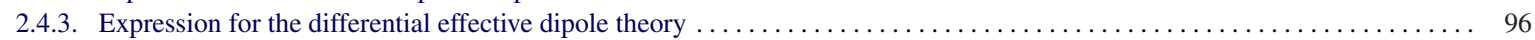

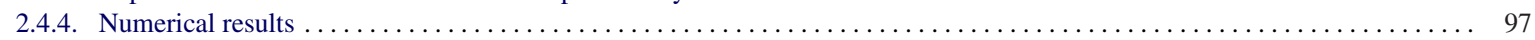

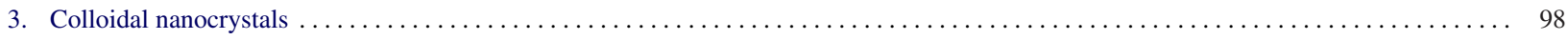

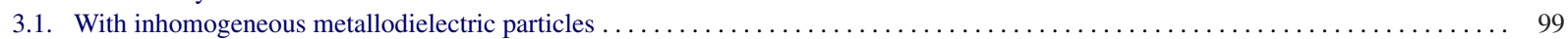

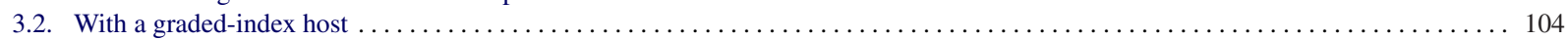

\footnotetext{
* Corresponding author. Tel.: +8621 55664928; fax: +8621 65104949.

E-mail address: jphuang@fudan.edu.cn (J.P. Huang).
} 


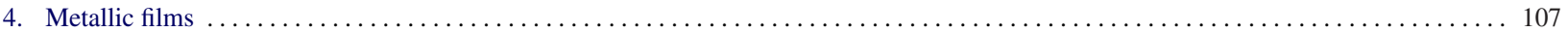

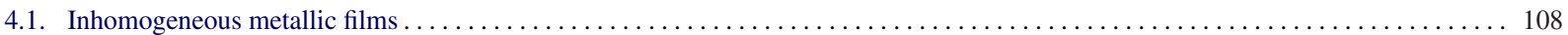

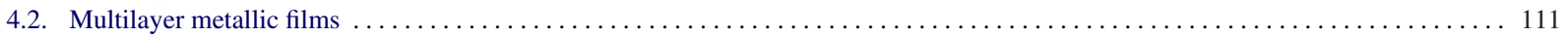

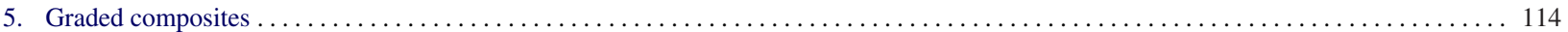

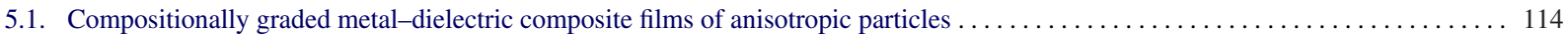

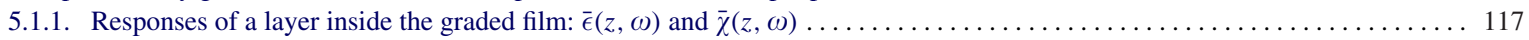

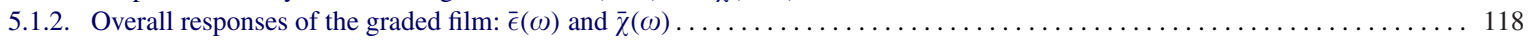

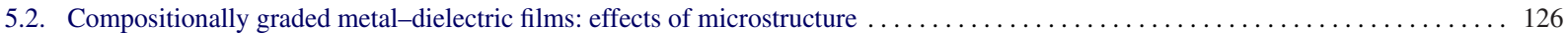

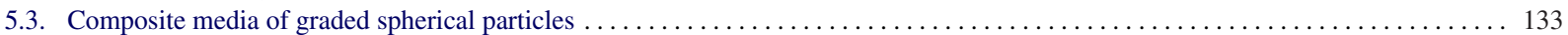

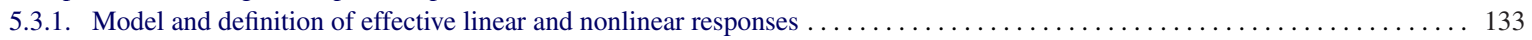

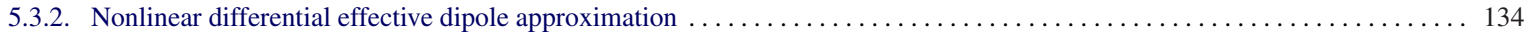

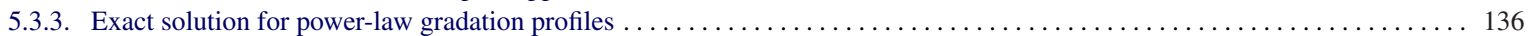

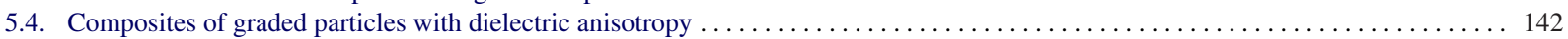

5.4.1. Model and definition of effective linear dielectric constant and third-order nonlinear susceptibility $\ldots \ldots \ldots \ldots \ldots \ldots \ldots$

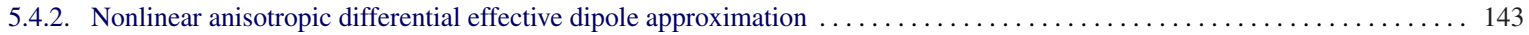

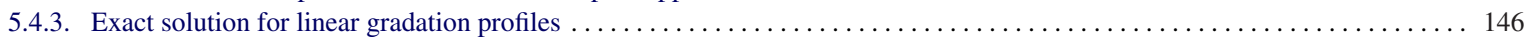

5.5. Spectral representation for understanding the effective dielectric constant of graded and multilayer composites $\ldots \ldots \ldots \ldots \ldots \ldots$

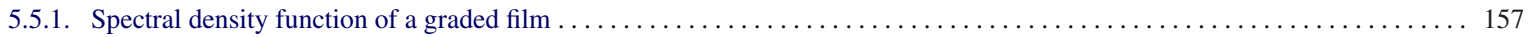

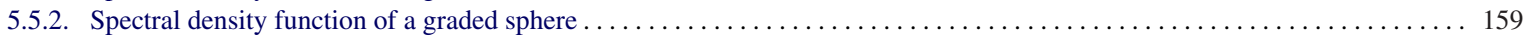

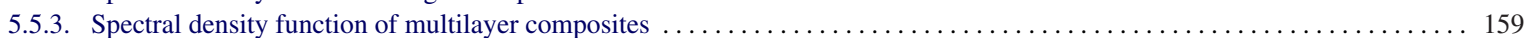

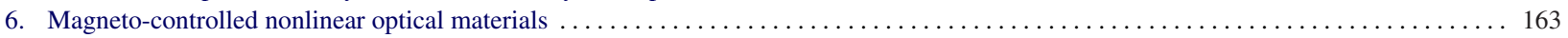

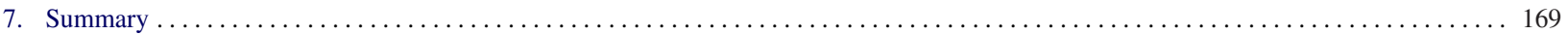

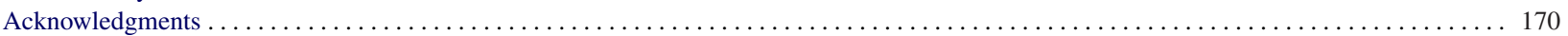

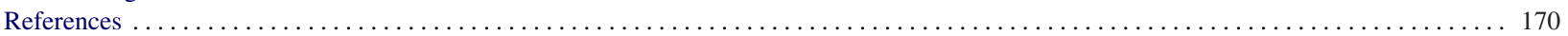

\section{Introduction}

The field of nonlinear optics traces its beginning to 1961, when a ruby laser was first used to generate the secondharmonic radiation inside a crystal [1]. Since then, nonlinear optics have become the basis of all the fledgling photonics technologies, where light works, or even replaces, electrons in applications traditionally carried out by microelectronics. The interaction between lights (electromagnetic fields) and matters is described by the Maxwell equations. The realization of all-optical switching, modulating and computing devices is an important goal in modern optical technology. The suitability of a material for these device applications requires a large magnitude of third-order nonlinear optical susceptibilities. In general, many applications of nonlinear optics that have been demonstrated under controlled laboratory conditions could become practical for technological uses if such materials were available. Thus, finding nonlinear optical materials with large nonlinear optical susceptibilities is up to now a challenge [2-10]. Many authors (e.g., see Refs. $[3,4,6,7,9,11]$ ) have devoted themselves to obtaining a large nonlinearity enhancement or optimal figure of merit (FOM) of bulk composites by taking into account various elements, such as the surface-plasmon resonance in metal-dielectric composites, structural information, etc. In the present review, the FOM denotes the ratio of a thirdorder nonlinear susceptibility to an optical absorption [12]. If there were a larger third-order nonlinear susceptibility and/or a smaller linear optical absorption under certain conditions, the corresponding FOM should be relatively larger, thus being attractive.

The most common way to achieve materials with a large nonlinear susceptibility is to use composite materials in which the constituent components possess large intrinsic nonlinear responses. Owing to composite effects, composite materials can have larger nonlinear susceptibilities at zero and finite frequencies than those of ordinary bulk materials or constituent materials from which the composite is constructed, which are thus called enhanced nonlinear optical responses. The formation of composite materials thus constitutes a means for engineering new materials with desired nonlinear optical properties [13]. In fact, the composite effects arise from strong enhancement or fluctuations of the local fields. One [14] studied nonlinear optical properties of fractal aggregates and showed that the aggregation of initially isolated particles into fractal clusters results in a huge enhancement of the nonlinear response within the spectral range of collective dipolar resonances like surface plasmon resonances. Basically, the response of a nonlinear composite can be tuned by controlling the volume fraction and morphology of constitutes. The latter can be purposefully adjusted by using external electric/magnetic field, or by adding dielectric gradation profile to the system of interest. In other 
words, depending on different microstructure, it is expected to achieve various kinds of composite effects on enhanced nonlinear optical responses.

When particles have a size that is much less than the wavelength of an incident light, the quasi-static approximation can be used to describe the response of an individual particle. (It should be remarked that all the approximations discussed in the article are valid in the quasi-static limit.) Typically, the particle size ranges from tens to hundreds of nanometers. The particles are embedded in a host material and can be aggregated into chains or clusters, under different conditions. It is important to keep in mind that, because of the symmetry requirement, all even order susceptibilities vanish when the material is centrosymmetric. In other words, odd order optical susceptibilities are possible in all systems. Noble metal (typically gold, silver and copper) is often chosen as an ingredient due to their extremely large and fast nonlinear optical responses. Many different microstructures have been exploited in an attempt to access the intrinsic optical nonlinearity of metals, for example, the random metallodielectric composites [15-17], fractal films [16,17], and alternative bilayers [4,5,18], etc. They basically rely on the enhanced local fields in space or on the effectively lengthened scale of the interactions between the matter and the incident light field. There is also a great demand for particular optical materials in devices applications, which would benefit from additional tunability of the optical properties. For example, recently we studied graded composites, which provided an extra degree of freedom for controlling the nonlinear optical properties of these materials by choosing appropriate gradation profiles [19-23]. In fact, there exist in Nature abundant graded materials, such as biological cells [24] and liquid crystal droplets [25]. Furthermore, many artificially-graded-index optical metamaterials and elements have been fabricated nowadays [26].

Composite effects are always expected to open a fascinating field of new phenomena in nonlinear optics, and the effects on the optical nonlinearity enhancement are different for various microstructures. This review presents our recent research aimed at understanding of the physical processes that determine the enhanced nonlinear optical properties of graded composite materials. This is an original, comprehensive, and first-handed review of the state-of-the-art development of the field of enhanced nonlinear optical responses in the materials. Regarding the previous development in the field, please refer to the excellent reviews by Shalaev and his coauthors [16,17], in which graded composites were not touched.

We shall review theories and models that have recently been devised to study the composite effect on enhanced nonlinear optical responses of various kinds of the recently-proposed nonlinear optical materials, namely, colloidal nanocrystals with inhomogeneous metallodielectric particles or a graded-index host (Section 3), metallic films with inhomogeneous microstructures adjusted by ion doping or temperature gradient (Section 4), composites with compositional gradation or graded particles (Section 5), and magneto-controlled ferrofluid-based nonlinear optical materials (Section 6). In addition, Section 2 also presents some fundamental theories that are to be used in the other sections. This review ends with a summary in Section 7.

\section{Fundamentals}

Composites often contain a macroscopic scale of inhomogeneity. In such a material, there are small, yet much larger than atomic, regions where macroscopic homogeneity prevails and where the foregoing macroscopic parameters suffices to characterize the physics, but different regions may have quite different values for those parameters. If we are interested in the physical properties at scales that are much larger than those regions and at which the material appears to be homogeneous, then the macroscopic behavior can again be characterized by bulk effective values, e.g. effective dielectric constant $\epsilon_{e}$. In what follows, we shall review three typical theories for calculating $\epsilon_{e}$, namely, the Maxwell-Garnett theory (Section 2.1), the Bruggeman theory (Section 2.2) as well as the Bergman-Milton spectral representation theory (Section 2.3).

Graded materials, whose material properties can vary continuously in space, are abundant in nature. With the advent of fabrication techniques, however, these materials may also be produced in laboratory to tailor their properties for specific need. Moreover, composites of graded inclusions can be more useful and interesting than those of homogeneous inclusions. However, the established theory for homogeneous inclusions cannot be applied directly. It is thus necessary to develop a new theory to study the effective properties of graded composite materials under externally applied fields. For this purpose, we shall develop an effective dipole theory (Section 2.4). 


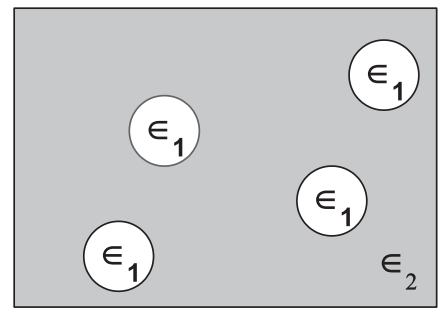

Fig. 1. Schematic graph showing an asymmetrical microstructure for the Maxwell-Garnett theory in which component 1 with dielectric constant $\epsilon_{1}$ is embedded in component 2 with $\epsilon_{2}$.

\subsection{The Maxwell-Garnett theory}

The Maxwell-Garnett theory [27,28] (or Maxwell-Garnett approximation) is also known as the Clausius-Mossotti theory. Regarding how to derive the equation for the Maxwell-Garnett theory, there are several approaches. Here we would like to start from the view of effective local electric field.

Let us discuss a two-component composite where many particles of the dielectric constant $\epsilon_{1}$ and the volume fraction $p$ are randomly embedded in a host medium of $\epsilon_{2}$, in the presence of an external electric field $E_{0}$ along the $z$-axis. Then, we denote the local electric field inside the particle by $E_{1}$, and that inside the host medium by $E_{2}$. Solving a fundamental equation in electrodynamics yields

$$
E_{1}=\frac{3 \epsilon_{2}}{\epsilon_{1}+2 \epsilon_{2}} E_{2}
$$

It is known that the average electric field $\langle E\rangle\left(\equiv p\left\langle E_{1}\right\rangle+(1-p)\left\langle E_{2}\right\rangle\right)$ inside the composite should be equal to the external electric field, namely, $\langle E\rangle=E_{0}$. On the other hand, the effective dielectric constant $\epsilon_{e}$ may be given by the ratio of the average displacement $\langle D\rangle$ to the average electric field $\langle E\rangle$ inside the composite, namely,

$$
\epsilon_{e}=\frac{\langle D\rangle}{\langle E\rangle}=\frac{\langle D\rangle}{E_{0}}
$$

Hence we obtain

$$
\epsilon_{e}=\frac{p \epsilon_{1}\left\langle E_{1}\right\rangle+(1-p) \epsilon_{2}\left\langle E_{2}\right\rangle}{p\left\langle E_{1}\right\rangle+(1-p)\left\langle E_{2}\right\rangle} .
$$

To this end, we obtain the expression for the Maxwell-Garnett theory as

$$
\epsilon_{e}=\epsilon_{2} \frac{\epsilon_{1}(1+2 p)+2 \epsilon_{2}(1-p)}{\epsilon_{1}(1-p)+\epsilon_{2}(2+p)} .
$$

We re-express Eq. (4) in a commonly-used form as

$$
\frac{\epsilon_{e}-\epsilon_{2}}{\epsilon_{e}+2 \epsilon_{2}}=p \frac{\epsilon_{1}-\epsilon_{2}}{\epsilon_{1}+2 \epsilon_{2}} \text {. }
$$

Obviously, the Maxwell-Garnett theory is an asymmetrical theory (see Fig. 1), namely the physical property of the composite can be changed if one exchanges the notations 1 and 2. Finally, for the Maxwell-Garnett theory the extension to multi-component composite is straightforward as can be easily done on the same footing.

\subsection{The Bruggeman theory}

Another approach to calculating $\epsilon_{e}$ for a two-component composite similar to the above was introduced by Bruggeman [29]. Thus, this approach is called the Bruggeman theory (also called the effective medium theory or Bruggeman approximation). Its predictions are usually sensible and physically offer a means of quick insight into some problems that are difficult to attack by other approaches. 


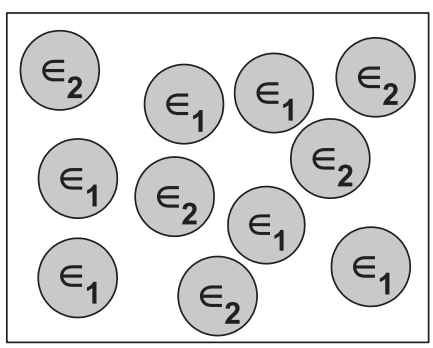

Fig. 2. Schematic graph showing a symmetrical microstructure for the Bruggeman theory in which two components are mixed.

We would derive the expression for the Bruggeman theory by considering the fact that the effective dipole factor $\langle b\rangle$ of the composite should be zero, namely

$$
\langle b\rangle=0 .
$$

On the other hand, we have

$$
\langle b\rangle=p b_{1}+(1-p) b_{2},
$$

where $b_{1}$ and $b_{2}$ are, respectively, the dipole factors of the particle and the host medium in the effective medium, and they are given by

$$
b_{1}=\frac{\epsilon_{1}-\epsilon_{e}}{\epsilon_{1}+2 \epsilon_{e}} \quad \text { and } \quad b_{2}=\frac{\epsilon_{2}-\epsilon_{e}}{\epsilon_{2}+2 \epsilon_{e}} .
$$

In view of Eqs. (6)-(8), we have directly the expression for the Bruggeman theory

$$
p \frac{\epsilon_{1}-\epsilon_{e}}{\epsilon_{1}+2 \epsilon_{e}}+(1-p) \frac{\epsilon_{2}-\epsilon_{e}}{\epsilon_{2}+2 \epsilon_{e}}=0 .
$$

Obviously, the Bruggeman theory is a symmetrical theory (see Fig. 2), namely the physical property of the composite can keep unchanged if one exchanges the notations 1 and 2. In addition, for the Bruggeman theory the extension to multi-component composite is also straightforward which can be readily done on the same footing as above.

\subsection{The Bergman-Milton spectral representation theory}

\subsubsection{Original derivation}

For the sake of convenience, we let $\mathbf{E}_{0}=-\hat{\mathbf{e}}_{z}$. Below we briefly review the Bergman-Milton spectral representation theory for the effective dielectric constant of a two-component composite.

The problem is initiated by solving the differential equation [30]

$$
\nabla \cdot\left[\left(1-\frac{1}{s} \eta(\mathbf{r})\right) \nabla \phi(\mathbf{r})\right]=0
$$

where $s=\epsilon_{2} /\left(\epsilon_{2}-\epsilon_{1}\right)$ denotes the relevant material parameter and $\eta(\mathbf{r})$ is the characteristic function of the composite, having value 1 for $r$ in the embedding medium and 0 otherwise. The electric potential $\phi(\mathbf{r})$ can be solved formally

$$
\phi(\mathbf{r})=z+\frac{1}{s} \int \mathrm{d} \mathbf{r}^{\prime} \eta\left(\mathbf{r}^{\prime}\right) \nabla^{\prime} G_{0}\left(\mathbf{r}-\mathbf{r}^{\prime}\right) \cdot \nabla^{\prime} \phi\left(\mathbf{r}^{\prime}\right),
$$

where $G_{0}\left(\mathbf{r}-\mathbf{r}^{\prime}\right)=\left|\mathbf{r}-\mathbf{r}^{\prime}\right| / 4 \pi$ is the free space Green's function. By denoting an integral-differential operator

$$
\Gamma=\int \mathrm{d} \mathbf{r}^{\prime} \eta\left(\mathbf{r}^{\prime}\right) \nabla^{\prime} G_{0}\left(\mathbf{r}-\mathbf{r}^{\prime}\right) \cdot \nabla^{\prime}
$$


and the corresponding inner product

$$
\langle\phi \mid \Phi\rangle=\int \mathrm{d} \mathbf{r} \eta(\mathbf{r}) \nabla \phi^{*} \cdot \nabla \Phi .
$$

It is easy to show that $\Gamma$ is a Hermitian operator. Let $s_{n}$ and $\Phi_{n}(\mathbf{r})$ be the $n$-th eigenvalue and eigenfunction of the $\Gamma$ operator, respectively, then we obtain the effective dielectric constant $\epsilon_{e}$ in the Bergman-Milton spectral representation

$$
\begin{aligned}
\epsilon_{e} & =-\frac{1}{V} \int \mathrm{d} V \epsilon(\mathbf{r}) E_{z} \\
& =\frac{1}{V} \int \mathrm{d} V \epsilon_{2}\left[1-\frac{1}{s} \eta(\mathbf{r})\right] \frac{\partial \Phi}{\partial z} \\
& =\epsilon_{2}\left(1-\frac{1}{V} \sum_{n} \frac{\left|\left\langle\Phi_{n} \mid z\right\rangle\right|^{2}}{s-s_{n}}\right) \\
& =\epsilon_{2}\left(1-\sum_{n} \frac{F_{n}}{s-s_{n}}\right) .
\end{aligned}
$$

The parameters $s_{n}$ and $F_{n}$ satisfy simple properties that $0 \leqslant s_{n} \leqslant 1$ and $\sum F_{n}=p$ [30]. Eq. (14) is just the effective dielectric constant of a two-component system in the Bergman-Milton spectral representation. Moreover, after introducing $F(s)$ as a function of $s$ as

$$
F(s) \equiv \sum_{n} \frac{F_{n}}{s-s_{n}},
$$

we may readily obtain the spectral structure of the composite. In doing so, we may further represent $F(s)$ as

$$
F(s)=\int_{0}^{1} \mathrm{~d} x \frac{\mu(x)}{s-x},
$$

where the spectral function $\mu(x)$ is a crucial parameter which contains the information about the spectral structure, and is thus given by

$$
\mu(x)=-\frac{1}{\pi} \operatorname{Im} F\left(x+\mathrm{i} 0^{+}\right) .
$$

Obviously, we may observe that the Bergman-Milton spectral representation is a rigorous mathematical formalism for the effective dielectric constant of a two-phase composite material [30]. It offers the advantage of the separation of materials parameters (namely the dielectric constant or conductivity) from the particle structure information (see Eq. (14)), thus simplifying the study.

For a better understanding of the Bergman-Milton spectral representation, below we would like to represent the above-mentioned Maxwell-Garnett and Bruggeman theories in the Bergman-Milton spectral representation. As a result, for the Maxwell-Garnett theory we respectively have the $F(s)$ function and the spectral function as

$$
F(s)=\frac{p}{s-(1-p) / 3} \text { and } \mu(x)=p \delta[x-(1-p) / 3] .
$$

On the other hand, for the Bruggeman theory we have the $F(s)$ function as

$$
F(s)=\frac{1}{4 s}\left(-1+3 p+3 s-3 \sqrt{\left(s-x_{1}\right)\left(s-x_{2}\right)}\right),
$$

where $x_{1}$ and $x_{2}$ are given by solving

$$
(1-3 p)^{2}-6(1+p) x+9 x^{2}=0,
$$




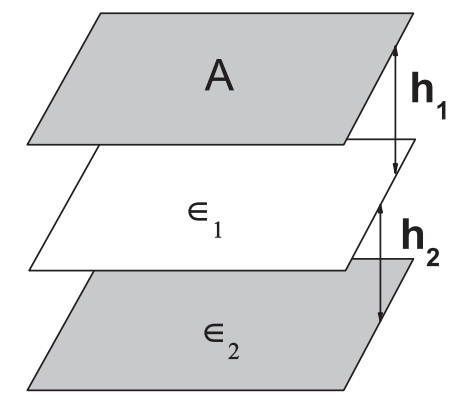

Fig. 3. Schematic graph showing a parallel-plate capacitor of total thickness $h=h_{1}+h_{2}$ that contains a dielectric slab of dielectric constant $\epsilon_{1}$ and thickness $h_{1}$, as well as a dielectric of $\epsilon_{2}$ and thickness $h_{2}$ (both of the same area $A$ ).

hence,

$$
x_{1}=\frac{1}{3}(1+p-2 \sqrt{2 p(1-p)}) \quad \text { and } \quad x_{2}=\frac{1}{3}(1+p+2 \sqrt{2 p(1-p)}) .
$$

In this case, the spectral function should be

$$
\mu(x)=\frac{3 p-1}{2} \theta(3 p-1)+\frac{3}{4 \pi x} \sqrt{\left(x-x_{1}\right)\left(x_{2}-x\right)}
$$

as $x_{1}<x<x_{2}$, and

$$
\mu(x)=\frac{3 p-1}{2} \theta(3 p-1),
$$

otherwise.

We would also like to mention that the extension of the Bergman-Milton spectral representation to the threecomponent composite can be made by taking into account various approaches [31-33].

\subsubsection{An easy-to-understand illustration of the Bergman-Milton spectral representation}

The essence of the spectral representation is to define the following transformations. If we denote a material parameter

$$
s=\left(1-\frac{\epsilon_{1}}{\epsilon_{2}}\right)^{-1},
$$

then the reduced effective dielectric constant

$$
w(s)=1-\frac{\epsilon_{e}}{\epsilon_{2}},
$$

can be written as

$$
w(s)=\sum_{n} \frac{F_{n}}{s-s_{n}},
$$

where $n$ is a positive integer, i.e., $n=1,2, \ldots$, and $F_{n}$ and $s_{n}$, are the $n$-th microstructure parameters of the composite materials [30]. In Eq. (20), $0 \leqslant s_{n}<1$ is a real number, while $F_{n}$ satisfies a sum rule [30]

$$
\sum_{n} F_{n}=p
$$

In what follows, we illustrate the spectral representation by the capacitance of simple geometry [34]. In particular, a parallel-plate capacitor is considered as an example. We will discuss two cases, namely, the series combination (Fig. 3) and the parallel combination (Fig. 4). 


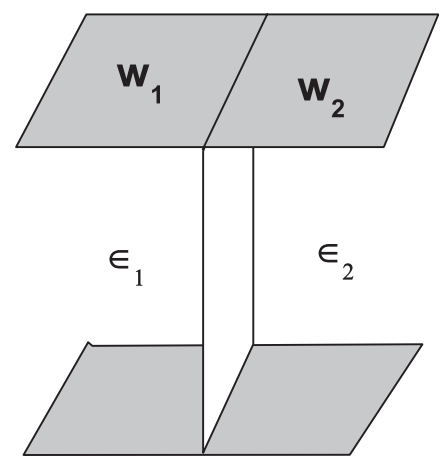

Fig. 4. Schematic graph showing a parallel-plate capacitor of total area $w=w_{1}+w_{2}$ that contains a dielectric slab of dielectric constant $\epsilon_{1}$ and area $w_{1}$, as well as a dielectric of $\epsilon_{2}$ and area $w_{2}$ (both of the same thickness $h$ ).

In the first case (see Fig. 3), if one inserts a dielectric slab of dielectric constant $\epsilon_{1}$ and thickness $h_{1}$, as well as a dielectric of $\epsilon_{2}$ and thickness $h_{2}$ (both of the same area $A$ ), into a parallel-plate capacitor of total thickness $h=h_{1}+h_{2}$, the overall capacitance $C$ is given by

$$
C^{-1}=C_{1}^{-1}+C_{2}^{-1}
$$

where $C_{1}=\epsilon_{1} A / h_{1}$ and $C_{2}=\epsilon_{2} A / h_{2}$. On the other hand, we may define the equivalent capacitance as $C=\epsilon_{e} A / h$, where $\epsilon_{e}$ is the effective dielectric constant. That is, we see the composite dielectric as a homogeneous dielectric of dielectric constant $\epsilon_{e}$.

Let $\epsilon_{1}=\epsilon_{2}(1-1 / s)$, we can express $C$ in the spectral representation,

$$
C=\frac{A \epsilon_{2}}{h}-\frac{A \epsilon_{2} h_{1} / h^{2}}{s-h_{2} / h} .
$$

In accord with the spectral representation, one may introduce $w(s)=1-\epsilon_{e} / \epsilon_{2}$, which is in fact the same as $w(s)=1-$ $C / C_{0}$, where $C_{0}$ is the capacitance when the plates are all filled with a dielectric material of $\epsilon_{2}$, namely $C_{0}=\epsilon_{2} A / h$. Thus we obtain

$$
w(s)=\frac{h_{1} / h}{s-h_{2} / h} .
$$

from which we find that the material parameter is separated from the geometric parameter. The comparison of $w(s)$ with Eq. (20) yields

$$
F_{1}=h_{1} / h, \quad s_{1}=h_{2} / h .
$$

It is worth noting that $F_{1}$ obtained herein is just equal to the volume fraction of the dielectric of $\epsilon_{1}$, and that $s_{1}$ satisfies $0 \leqslant s_{1}<1$, as required by the spectral representation theory.

Next, we consider the parallel combination (see Fig. 4). If one inserts a material with dielectric constant $\epsilon_{1}$ and area $w_{1}$ as well as a dielectric with $\epsilon_{2}$ and area $w_{2}$ (both of the same thickness $h$ ), into a parallel-plate capacitor of total area $A=w_{1}+w_{2}$, the overall capacitance $C$ is given by

$$
C=C_{1}+C_{2},
$$

where $C_{1}=\epsilon_{1} w_{1} / h$ and $C_{2}=\epsilon_{2} w_{2} / h$. Similarly, after introducing the effective dielectric constant $\epsilon_{e}$, we may define the overall capacitance as $C=\epsilon_{e} A / h$. 


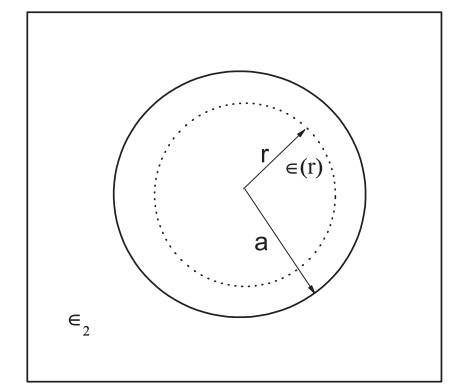

Fig. 5. Schematic graph showing a graded spherical particle with a dielectric gradation profile $\epsilon(r)$ at radius $r$, embedded in a host medium of dielectric constant $\epsilon_{2}$.

Again, in the spectral representation, let $s=\left(1-\epsilon_{1} / \epsilon_{2}\right)^{-1}$, then

$$
C=\frac{\epsilon_{2} A}{h}-\frac{\epsilon_{2} w_{1}}{h s}
$$

Writing $w(s)=1-C / C_{0}$, we obtain

$$
w(s)=\frac{w_{1} / A}{s} .
$$

From this equation, the material parameter is also found to be separated from the geometric parameter. It is clear that $F_{1}=w_{1} / A$, i.e., the volume fraction of the dielectric of $\epsilon_{1}$, and $s_{1}=0$.

\subsection{The differential effective dipole theory}

\subsubsection{Dipole moment of a graded spherical particle}

We consider an inhomogeneous spherical inclusion of radius $a$, with a dielectric gradation profile $\epsilon(r)$, embedded in a host medium of dielectric constant $\epsilon_{2}$ (see Fig. 5). In a uniform applied field $E_{0}$, the potential functions in the inclusion and host regions can be obtained by solving the Laplace equation with the appropriate boundary conditions. For a power-law dielectric profile $\epsilon(r)=A \epsilon_{2} r^{n}$, where $A, n$ are constants, the potential admits an exact solution [19]:

$$
\begin{aligned}
& \phi(r)=-\xi_{1} E_{0} r^{s} \cos \theta, \quad r<a, \\
& \phi(r)=-E_{0} r \cos \theta+\frac{\xi_{2}}{r^{2}} E_{0} \cos \theta, \quad r>a,
\end{aligned}
$$

where the coefficients $\xi_{1}$ and $\xi_{2}$ have the form

$$
\xi_{1}=\frac{3 a^{1-s}}{s A a^{n}+2}, \quad \xi_{2}=\frac{s A a^{n}-1}{s A a^{n}+2} a^{3},
$$

and $s$ is given by the positive root of a quadratic equation [19]:

$$
s=\frac{1}{2}\left(\sqrt{9+2 n+n^{2}}-(1+n)\right) .
$$

The local electric field can be computed by the gradient of the potential $\mathbf{E}=-\nabla \phi$. From the local electric field, we can integrate it over the volume of the inclusion to obtain the dipole moment of a graded inclusion. Thus we find an exact analytic expression for the dipole moment in the power-law profile [19]:

$$
p_{0}=\epsilon_{2} b E_{0} a^{3},
$$

where $b$ is the dipole factor, which measures the degree of polarization of the inclusion in an external field,

$$
b=\frac{s+2}{s A a^{n}+2}\left(\frac{A a^{n}}{s+n+2}-\frac{1}{s+2}\right) .
$$


Note that when $n \rightarrow 0$, the dielectric profile becomes homogeneous. In this limit $s \rightarrow 1$, Eq. (26) reduces to the well-known result for the dipole factor of a homogeneous spherical inclusion of dielectric constant $\epsilon_{1}$ embedded in a host medium of $\epsilon_{2}$ :

$$
b_{0}=\frac{\epsilon_{1}-\epsilon_{2}}{\epsilon_{1}+2 \epsilon_{2}} .
$$

\subsubsection{Dipole moment of a coated spherical particle}

If we add to the homogeneous inclusion a spherical shell of dielectric constant $\epsilon$, to make a coated sphere [35] of overall radius $a_{1}$. By solving the Laplace equation with appropriate boundary conditions on the interfaces, the potential in the inclusion becomes [36]

$$
\begin{aligned}
& \phi(r)=-\xi E_{0} r \cos \theta, \quad r<a, \\
& \phi(r)=-E_{0}\left(\xi_{3} r-\frac{\xi_{4}}{r^{2}}\right) \cos \theta, \quad a<r<a_{1},
\end{aligned}
$$

where $\xi, \xi_{3}$ and $\xi_{4}$ are coefficients in terms of the dielectric constants [36]. The local electric field is obtained and hence yields the dipole moment of a single coated spherical inclusion:

$$
p_{1}=\epsilon_{2} b_{1} E_{0} a_{1}^{3},
$$

where $b_{1}$ is the dipole factor of the coated sphere [37],

$$
b_{1}=\frac{\left(\epsilon-\epsilon_{2}\right)+\left(\epsilon_{2}+2 \epsilon\right) \rho_{1} y}{\left(\epsilon+2 \epsilon_{2}\right)+2\left(\epsilon-\epsilon_{2}\right) \rho_{1} y},
$$

where $\rho_{1}$ is given by

$$
\rho_{1}=\frac{\epsilon_{1}-\epsilon}{\epsilon_{1}+2 \epsilon},
$$

and

$$
y=\left(a / a_{1}\right)^{3} .
$$

In Eq. (31) $\epsilon_{1}$ denotes the dielectric constant of the core inside the coated spherical particle. The consideration can be extended to more shells of different dielectric constants, at the expense of more complicated expressions [37-39]. It is easy to check that $b_{1}$ reduces to $b_{0}$ when $\epsilon=\epsilon_{1}$. Thus, the dipole factor remains unchanged if one adds a spherical shell of the same dielectric constant. We should remark that these exact results are only available for a few simple dielectric profiles, and it is our objective in the following section to develop a theory for any arbitrary profiles.

\subsubsection{Expression for the differential effective dipole theory}

Now we develop the differential effective dipole theory (DEDT) for spherical particles of graded materials and hence compute the effective dielectric response in the dilute limit. To establish the differential effective dipole theory, we mimic the graded profile by a multi-shell construction, i.e., we build up the dielectric profile gradually by adding shells. We start with an infinitesimal spherical core of dielectric constant $\epsilon(0)$ and keep on adding spherical shells of dielectric constant given by $\epsilon(r)$ at radius $r$, until $r=a$ is reached. At radius $r$, we have an inhomogeneous sphere whose induced dipole moment is given by $p(r)$. Certainly $p(r)$ is proportional to $E_{0}$, but the exact expression is lacking. We further replace the inhomogeneous sphere by a homogeneous sphere of the same dipole moment and the graded profile is replaced by an effective dielectric constant $\bar{\epsilon}(r)$. Thus,

$$
p(r)=\epsilon_{2} b(r) E_{0} r^{3},
$$

where

$$
b(r)=\frac{\bar{\epsilon}(r)-\epsilon_{2}}{\bar{\epsilon}(r)+2 \epsilon_{2}} .
$$


Next, we add to the sphere a spherical shell of infinitesimal thickness $\mathrm{d} r$, of dielectric constant $\epsilon(r)$. The dipole factor will change according to Eq. (30). Of course, the effective dielectric constant $\bar{\epsilon}(r)$, being related to $b(r)$, should also change by the same token. Let us write $b_{1}=b+\mathrm{d} b$, and take the limit $\mathrm{d} r \rightarrow 0$, we obtain a differential equation:

$$
\frac{\mathrm{d} b}{\mathrm{~d} r}=-\frac{1}{3 r \epsilon_{2} \epsilon(r)}\left[(1+2 b) \epsilon_{2}-(1-b) \epsilon(r)\right]\left[(1+2 b) \epsilon_{2}+2(1-b) \epsilon(r)\right] .
$$

Thus the dipole factor of a graded spherical particle can be calculated by solving the above differential equation with a given graded profile $\epsilon(r)$. The nonlinear first-order differential equation can be integrated, at least numerically, if we are given the graded profile $\epsilon(r)$ and the initial condition $b(r=0)$. When $b(r=a)$ is calculated, we can compute the dipole moment of individual spherical particles and hence the effective dielectric constant either in the dilute limit

$$
\epsilon_{e}=\epsilon_{2}+3 f \epsilon_{2} b
$$

or by the Clausius-Mossotti approximation

$$
\frac{\epsilon_{e}-\epsilon_{2}}{\epsilon_{e}+2 \epsilon_{2}}=f b
$$

where $f$ is the volume fraction of spherical particles.

\subsubsection{Numerical results}

In this section, we evaluate the DEDT for some graded profiles. We performed numerical calculations for two model profiles: (a) power-law profile $\epsilon(r)=A r^{n}$, and (b) linear profile $\epsilon(r)=A+B r$. Without loss of generality, we have set $\epsilon_{2}=1$ and $a=1$. The numerical integration has been done by the fourth-order Runge-Kutta algorithm with a step size $\delta r=0.01$, starting with a small core radius $r=0.01$. In Fig. 6(a), we plot the dipole factor $b$ versus $A$ for various index $n>0$. It is clear that $b$ increases monotonically as the dielectric contrast $A$ increases, while it decreases with the index $n$. It is attributed to the fact that $\bar{\epsilon}$ decreases as $n$ increases. Similarly, in Fig. 6(b) we plot $b$ versus $A$ for various slope $B$. We obtained similar behavior as in Fig. 6(a).

It is instructive to compare the exact results with the DEDT results. In Fig. 7, we compared the exact results and the DEDT results for a spherical inclusion with a power-law graded profile. The agreement is excellent for positive index $n$. However, we doubted if the comparison remains good for negative index $n$ because the dielectric constant diverges at the origin. To our surprise, the agreement is still excellent for negative index $n$ (results not shown here). In fact, the DEDT is exact for the case of a spherical inclusion with a power-law profile. We have checked the exact solution [Eq. (26)] against the DEDT. We found that the differential equation [Eq. (35)] as well as the boundary condition at a small radius are satisfied by Eq. (26). Thus, the DEDT is exact for power-law graded spherical inclusions.

The DEDT is better motivated by the following considerations. In the case of a single-shell inclusion of core dielectric constant $\epsilon_{1}$, coated by a shell of dielectric constant $\epsilon$, with the core to whole volume ratio $y$ (as the one considered in Section 3), the effective dielectric constant of the inclusion $(\bar{\epsilon})$ may be determined by the Maxwell-Garnett approximation.

$$
\frac{\bar{\epsilon}-\epsilon}{\bar{\epsilon}+2 \epsilon}=y \frac{\epsilon_{1}-\epsilon}{\epsilon_{1}+2 \epsilon}
$$

Then, we assume the effective inclusion of dielectric constant $\bar{\epsilon}$ to be embedded in a host medium $\epsilon_{2}$. In this way, the dipole factor of the effective inclusion can be calculated from

$$
b=\frac{\bar{\epsilon}-\epsilon_{2}}{\bar{\epsilon}+2 \epsilon_{2}} .
$$

For a single-shell inclusion, this consideration is exact, in the sense that Eq. (30) is reproduced exactly, while for multi-shell inclusions, this can be a good approximation.

Moreover, the substitution of Eq. (34) into Eq. (35) yields a differential equation for $\bar{\epsilon}(r)$ :

$$
\frac{\mathrm{d}}{\mathrm{d} r}[r \bar{\epsilon}(r)]+\frac{[\bar{\epsilon}(r)]^{2}}{\epsilon(r)}=2 \epsilon(r),
$$




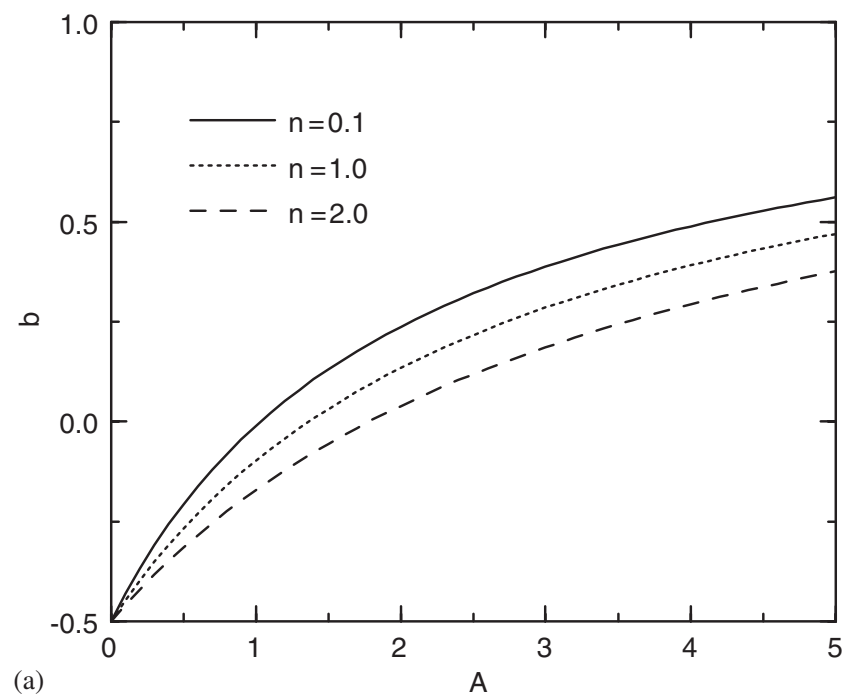

(a)

A

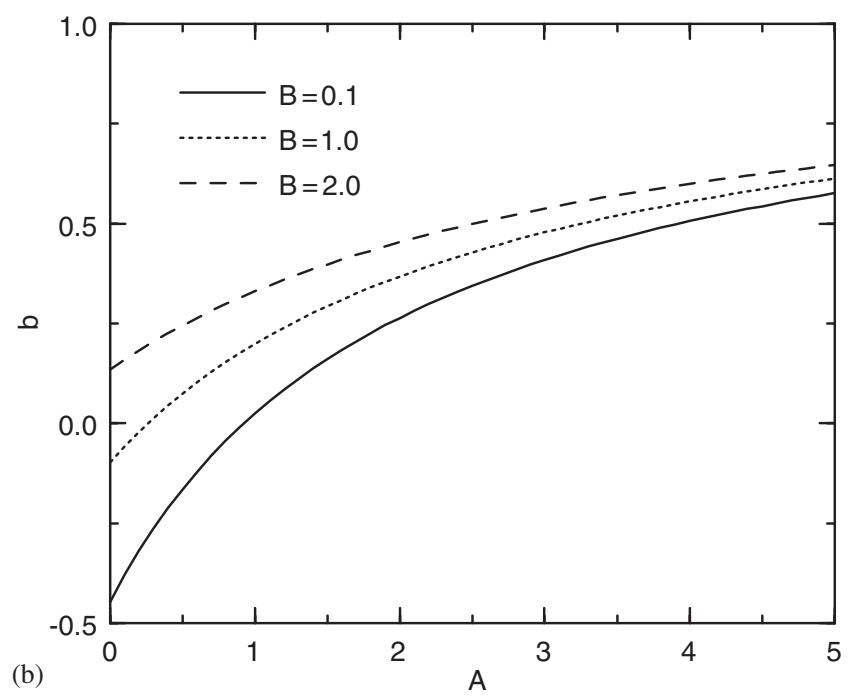

Fig. 6. The dipole factor $b$ plotted versus $A$ for two model profiles for: (a) various index $n$ in the power-law profile; and (b) various slope $B$ in the linear profile.

which coincides with the Tartar formula [39] derived for assemblages of spheres with varying radial and tangential conductivity. We should remark that the DEDT is also able to offer a good agreement with the exact result of a linear profile [19]. Most importantly, we have recently demonstrated that the DEDT is indeed exact for graded spherical particles of arbitrary dielectric gradation profiles [40].

\section{Colloidal nanocrystals}

Colloidal crystalline is extensively studied in nanomaterials engineering and its potential applications range from nanophotonics to chemistry and biomedicine [41]. Colloidal crystals can be prepared via templated sedimentation, methods based on capillary forces, and electric fields [42-44]. They exhibit body centered tetragonal (bct), body centered cubic (bcc) and face-centered cubic (fcc) structures, depending on the lattice constants and hence the volume fraction of colloidal particles. These structures can be investigated by using static and dynamic light scattering techniques $[45,46]$. 


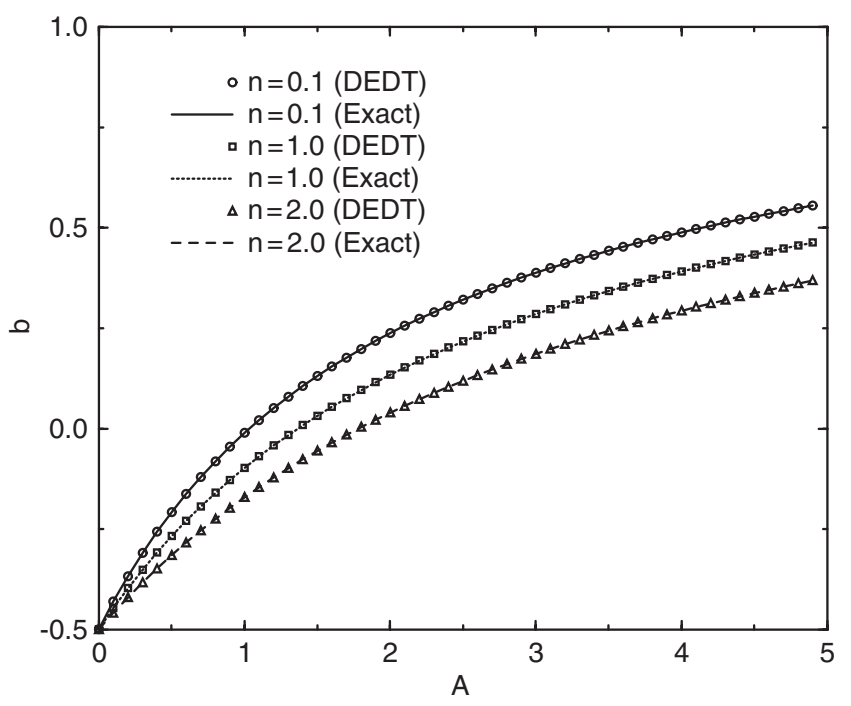

Fig. 7. Similar to Fig. 6(a), but for comparison of the exact results (lines) with differential effective dipole theory results (symbols) for the dipole factor in the power-law profile.

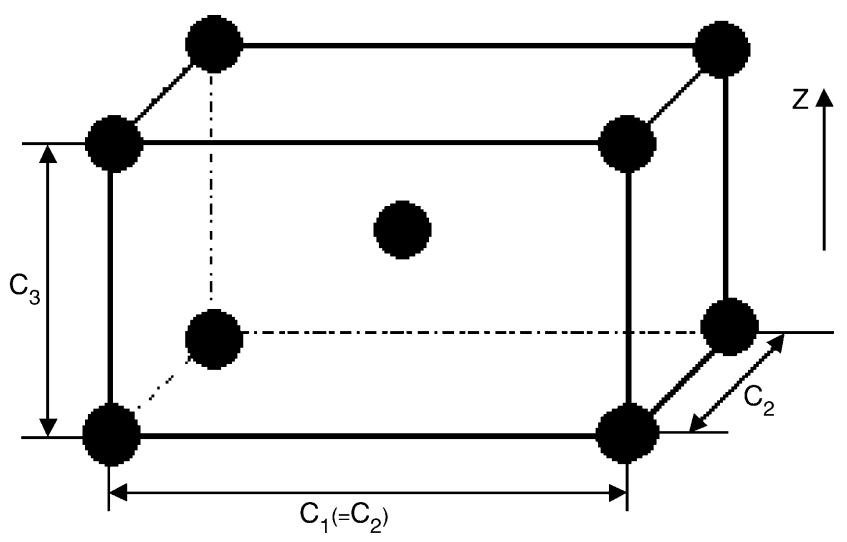

Fig. 8. Schematic graph to show the location of two colloidal nanoparticles in a tetragonal unit cell [49].

So far, colloidal-based optical sensors [47] (and photonic-band-gap materials based on inverse opaline structures [48]) have been made possible by these fabrication techniques.

This section describes a class of colloidal-crystal-based nonlinear optical materials, which are made of graded metallodielectric nanoparticles (namely, a graded metallic core plus a dielectric shell), or a graded-index host.

\subsection{With inhomogeneous metallodielectric particles}

Let us start by considering a tetragonal unit cell which has a basis of two colloidal nanoparticles each of which is fixed with an induced point dipole at its center. One of the two nanoparticles is located at a corner and the other one at the body center of the cell (Fig. 8). Its lattice constants are denoted by $c_{1}\left(=c_{2}\right)=\ell q^{-1 / 2}$ and $c_{3}=\ell q$ along $x(y)$ and $z$ axes, respectively. In this case, the uniaxial anisotropic axis is directed along $z$ axis. The degree of anisotropy of the periodic lattice is measured by how $q$ deviates from unity. In particular, $q=0.87358,1.0$ and $2^{1 / 3}$ represents the bct, bcc and fec lattice, respectively. In general, for a colloidal crystal, the individual colloidal nanoparticles should be 
touching. In fact, a colloidal crystal without the particles' touching can also be made if the colloidal nanoparticles are charged and stabilized by electrostatic forces. Below we shall investigate colloidal crystals with the particles' touching.

With recent advancements in the fabrication of nanoshells [50,51], we are allowed to use a dielectric surface layer with thickness $d$ on an inhomogeneous (graded) metallic core with radius $a$, in order to activate repulsive (or attractive) force between the nanoparticles. This is also a crucial requirement because otherwise multipolar interaction between the metallic cores can be important. The dielectric constant $\epsilon_{1}(r)(r \leqslant a)$ of the metallic core should be a radial function, because of a radial gradation. The dielectric constant $\epsilon_{s}$ of the surface layer can be the same as that $\epsilon_{2}$ of the host fluid, as to be used in the following. In this regard, the surface layer contributes to the geometric constraint

$$
c_{1}^{2}+c_{2}^{2}+c_{3}^{2}=16(a+d)^{2},
$$

rather than the effective optical responses. Owing to this constraint, it is found that the smallest $q$ occurs at the bct lattice while the largest $q$ occurs at the fcc. Meanwhile, we obtain a relation between $q$ and the volume fraction $p$ of the metallic component,

$$
p=\frac{\pi}{24 t^{3}}\left(\frac{q^{3}+2}{q}\right)^{3 / 2}
$$

with thickness parameter $t=(a+d) / a$. When an external electric field $\mathbf{E}_{0}$ is applied along $x$ axis, the induced dipole moment $\mathbf{P}$ are perpendicular to the uniaxial anisotropic axis. Then, the local field $\mathbf{E}_{\mathrm{L}}$ at the lattice point $\mathbf{R}=\mathbf{0}$ can be determined by using the Ewald-Kornfeld formulation [52-54],

$$
E_{\mathrm{L}}=P \sum_{j=1}^{2} \sum_{\vec{R} \neq \overrightarrow{0}}\left[-\gamma_{1}\left(R_{j}\right)+x_{j}^{2} q^{2} \gamma_{2}\left(R_{j}\right)\right]-\frac{4 \pi P}{V_{c}} \sum_{\vec{G} \neq \overrightarrow{0}} \Pi(\vec{G}) \frac{G_{x}^{2}}{G^{2}} \exp \left(\frac{-G^{2}}{4 \eta^{2}}\right)+\frac{4 P \eta^{3}}{3 \sqrt{\pi}} .
$$

Here we have taken into account the influence of the local-field effect arising from all the other particles in the lattice (lattice effect). In Eq. (40), $\gamma_{1}$ and $\gamma_{2}$ are two coefficients, given by

$$
\begin{aligned}
& \gamma_{1}(r)=\left[\operatorname{erfc}(\eta r) / r^{3}\right]+\left(2 \eta / \sqrt{\pi} r^{2}\right) \exp \left(-\eta^{2} r^{2}\right), \\
& \gamma_{2}(r)=\left[3 \operatorname{erfc}(\eta r) / r^{5}\right]+\left[4 \eta^{3} /\left(\sqrt{\pi} r^{2}\right)+6 \eta /\left(\sqrt{\pi} r^{4}\right)\right] \exp \left(-\eta^{2} r^{2}\right),
\end{aligned}
$$

where $\operatorname{erfc}(\eta r)$ is the complementary error function, and $\eta$ an adjustable parameter making the summation converge rapidly. $R$ and $G$ denote, respectively, the lattice vector and the reciprocal lattice vector,

$$
\begin{aligned}
& \vec{R}=\ell\left(q^{-1 / 2} l \hat{x}+q^{-1 / 2} m \hat{y}+q n \hat{z}\right), \\
& \vec{G}=(2 \pi / \ell)\left(q^{1 / 2} u \hat{x}+q^{1 / 2} v \hat{y}+q^{-1} w \hat{z}\right),
\end{aligned}
$$

where $l, m, n, u, v$, and $w$ are integers. In addition, $x_{j}$ and $R_{j}$ are given by

$$
\begin{aligned}
& x_{j}=l-(j-1) / 2, \\
& R_{j}=|\vec{R}-[(j-1) / 2](a \hat{x}+a \hat{y}+c \hat{z})|,
\end{aligned}
$$

and the structure factor $\Pi(\vec{G})=1+\exp [\mathrm{i}(u+v+w) / \pi]$. Now we define a local field factor in transverse field cases, $\alpha_{\perp}=3 V_{c} E_{\mathrm{L}} /(4 \pi P)$. It is worth noting that $\alpha_{\perp}$ is a function of a single variable $q$. Also, there is a sum rule $[52,55]$

$$
2 \alpha_{\perp}+\alpha_{\|}=3,
$$

where $\alpha_{\|}$denotes the local field factor in longitudinal field cases. Here the longitudinal (or transverse) field case corresponds to the fact that the $E$ field of the incident light is parallel (or perpendicular) to the uniaxial anisotropic $z$ axis. For the bct, bcc and fcc lattices, we obtain $\alpha_{\perp}=0.95351,1.0$, and 1.0 (or alternatively $\alpha_{\|}=1.09298,1.0$, and 1.0), respectively. If there are no special instructions, we shall use $\alpha$ to denote both $\alpha_{\perp}$ and $\alpha_{\|}$in the following. Next, for obtaining the effective dielectric constant $\epsilon_{e}$ (which also indicates both $\epsilon_{e}^{\perp}$ and $\epsilon_{e}^{\|}$, the effective dielectric 
constants in transverse and longitudinal field cases, respectively) of the colloidal crystal, we resort to the anisotropic Maxwell-Garnett formula [9] with a high degree of accuracy [56] due to the explicit determination of $\alpha$,

$$
\frac{\epsilon_{e}-\epsilon_{2}}{\alpha \epsilon_{e}+(3-\alpha) \epsilon_{2}}=p \frac{\bar{\epsilon}_{1}-\epsilon_{2}}{\bar{\epsilon}_{1}+2 \epsilon_{2}}
$$

where the equivalent dielectric constant $\bar{\epsilon}_{1} \equiv \bar{\epsilon}_{1}(r=a)$ for the graded metallic core can be obtained [20,39] by solving

$$
\mathrm{d} \bar{\epsilon}_{1}(r) / \mathrm{d} r=\left[\epsilon_{1}(r)-\bar{\epsilon}_{1}(r)\right]\left[\bar{\epsilon}_{1}(r)+2 \epsilon_{1}(r)\right] /\left[r \epsilon_{1}(r)\right],
$$

as long as the gradation profile $\epsilon_{1}(r)$ is given. Assuming both the host fluid and dielectric surface layer to be linear for convenience, the effective third-order nonlinear susceptibility $\chi_{e}^{(3)}$ for the graded colloidal crystal is given by

$$
\chi_{e}^{(3)}=p \frac{\left\langle\left|\mathbf{E}_{\text {lin }}\right|^{2}\right\rangle\left\langle\mathbf{E}_{\text {lin }}^{2}\right\rangle}{\left|\mathbf{E}_{0}\right|^{2} \mathbf{E}_{0}^{2}} \bar{\chi}_{1}^{(3)}
$$

under a decoupling approximation, where the equivalent third-order nonlinear susceptibility $\bar{\chi}_{1}^{(3)} \equiv \bar{\chi}_{1}^{(3)}(r=a)$ for the graded metallic core can be obtained [20] by solving

$$
\begin{aligned}
\mathrm{d} \bar{\chi}_{1}^{(3)}(r) / \mathrm{d} r= & \bar{\chi}_{1}^{(3)}(r)\left\{\left(3 \mathrm{~d} \bar{\epsilon}_{1}(r) / \mathrm{d} r\right) /\left(2 \epsilon_{2}+\bar{\epsilon}_{1}(r)\right)+\left[\left(\mathrm{d} \bar{\epsilon}_{1}(r) / \mathrm{d} r\right) /\left(2 \epsilon_{2}+\bar{\epsilon}_{1}(r)\right)\right]^{*}\right\} \\
& +\bar{\chi}_{1}^{(3)}(r)\left(6 Y+2 Y^{*}-3\right) / r+3 \chi_{1}^{(3)}(r) /(5 r) \\
& \times\left|\left(\bar{\epsilon}_{1}(r)+2 \epsilon_{1}(r)\right) /\left(3 \epsilon_{1}(r)\right)\right|^{2}\left[\left(\bar{\epsilon}_{1}(r)+2 \epsilon_{1}(r)\right) /\left(3 \epsilon_{1}(r)\right)\right]^{2} \\
& \times\left(5+18 X^{2}+18|X|^{2}+4 X^{3}+12 X|X|^{2}+24|X|^{2} X^{2}\right),
\end{aligned}
$$

with

$$
\begin{aligned}
& X=\frac{\bar{\epsilon}_{1}(r)-\epsilon_{1}(r)}{\bar{\epsilon}_{1}(r)+2 \epsilon_{1}(r)} \\
& Y=\frac{\left[\epsilon_{1}(r)-\epsilon_{2}\right]\left[\bar{\epsilon}_{1}(r)-\epsilon_{1}(r)\right]}{\epsilon_{1}(r)\left[\bar{\epsilon}_{1}(r)+2 \epsilon_{2}\right]},
\end{aligned}
$$

as long as the gradation profile $\chi_{1}^{(3)}(r)$ is also given. Here the intrinsic weak third-order nonlinear susceptibility $\chi_{1}^{(3)}(r)$ satisfies the local constitutive relation between the displacement $\mathbf{D}_{1}(r)$ and the electric field $\mathbf{E}_{1}(r)$ inside the graded metallic core,

$$
\mathbf{D}_{1}(r)=\epsilon_{1}(r) \mathbf{E}_{1}(r)+\chi_{1}^{(3)}(r)\left|\mathbf{E}_{1}(r)\right|^{2} \mathbf{E}_{1}(r) .
$$

In Eq. (50), $\langle\cdots\rangle$ denotes the volume average over the metallic region, and $\mathbf{E}_{\text {lin }}$ the equivalent linear local electric field in the graded metallic core with the same gradation profile but with a vanishing nonlinear response at the frequency concerned. Both $\left\langle\left|\mathbf{E}_{\text {lin }}\right|^{2}\right\rangle$ and $\left\langle\mathbf{E}_{\text {lin }}^{2}\right\rangle$ can be expressed in the spectral representation as [57]

$$
\begin{aligned}
& \left\langle\left|\mathbf{E}_{\text {lin }}\right|^{2}\right\rangle=\left(\mathbf{E}_{0}^{2} / p\right) \int \mathrm{d} x^{\prime}|s|^{2} \mu\left(x^{\prime}\right) /\left|s-x^{\prime}\right|^{2}, \\
& \left\langle\mathbf{E}_{\text {lin }}^{2}\right\rangle=\left(\mathbf{E}_{0}^{2} / p\right) \int \mathrm{d} x^{\prime} s^{2} \mu\left(x^{\prime}\right) /\left(s-x^{\prime}\right)^{2},
\end{aligned}
$$

with material parameter $s=\epsilon_{2} /\left(\epsilon_{2}-\bar{\epsilon}_{1}\right)$, where $\mu\left(x^{\prime}\right)$ is the spectral density. Since the $\epsilon_{e}$ in Eq. (48) can be expressed as

$$
\epsilon_{e}=\epsilon_{2}\left[1-\frac{p}{s-(1-p \alpha) / 3}\right] \equiv \epsilon_{2}[1-F(s)],
$$

according to

$$
\mu\left(x^{\prime}\right)=-\frac{1}{\pi} \operatorname{Im}\left[F\left(x^{\prime}+\mathrm{i} 0^{+}\right)\right]
$$


we obtain

$$
\mu\left(x^{\prime}\right)=p \delta\left(x^{\prime}-\frac{1-p \alpha}{3}\right) .
$$

Here $\operatorname{Im}[\cdots]$ denotes the imaginary part of $\cdots$.

The point of achieving the resonant plasma band shown in Figs. 9 and 10 is that one needs a sufficiently large gradient rather than a crucially particular form of the dielectric function or gradation profiles. To show the effects of gradation, we have adopted the Drude form

$$
\epsilon_{1}(r)=1-\frac{\omega_{p}^{2}(r)}{\omega(\omega+\mathrm{i} \gamma)}
$$

with a model plasma-frequency gradation profile

$$
\omega_{p}(r)=\omega_{p}(0)\left(1-C_{\omega} \frac{r}{a}\right) .
$$

One possible way to achieve such gradation is to fabricate a graded metallic core by using different noble metals as different layers inside the core. For focusing on the nonlinearity enhancement, we consider a model system where $\chi_{1}^{(3)}(r)=\chi_{1}^{(3)}$ is a real and positive frequency-independent constant and does not have a gradation profile. In this case, the equivalent nonlinear susceptibility $\bar{\chi}_{1}^{(3)}(r)$ should still depend on $r$ because of the radial function $\epsilon_{1}(r)$ [20].

For a given thickness of shell, when $q$ varies from bct to bcc, to fcc lattices, the volume fraction $p$ first decreases from bct, reaches a minimum at bcc, then increases again towards fcc (Table 1). At the same time, the longitudinal local field factor $\alpha_{\|}$decreases from 1.09 at bct lattices almost monotonically to 1 at bcc and fcc lattices. Thus, for the bct case, the large $p$ and large $\alpha_{\|}$should give rise to a large red shift (namely, the plasma resonant peak and band are caused to be located at lower frequencies) from the single particle case with $p \approx 0$ where the lattice effect disappears and only the gradation effect exists. For bcc lattices, the red shift should be the smallest due to small $p$ and $\alpha_{\|}=1$, and hence for fcc lattices, the red shift should lie between those of bct and bct lattices. This is because $\alpha$ does not change much while $p$ changes significantly (Table 1). From Fig. 9, it is evident that for a given lattice, the effective linear and nonlinear responses depend strongly on the thickness parameter $t$. Both the redshift and strength of the plasma resonant peak or band is largest at smallest $t$ [Fig. 9(a)-(b)]. This is a combination of the local field effect and the volume fraction effect in the colloidal crystal. However, for a given thickness parameter $t$, the dependence of these responses on the crystal structure is not prominent (no figures shown here). In fact, the plasma resonant band in Fig. 9 is caused to appear by the gradation, as discussed in Ref. [21] in which the results for the case of various $C_{\omega}$ (and hence various degree of gradation) have also been reported. Similar results are displayed in Fig. 10 where the transverse field cases are investigated. In comparison with the longitudinal field cases (Fig. 9), the framework of the responses in transverse field cases (Fig. 10) is slightly blue-shifted (i.e., located at higher frequency). Owing to the volume fraction effect, this behavior is more evident for the smallest $t$, but small enough to be neglected at the largest $t$. The difference between the results predicted in Figs. 9 and10 is generally small because $\alpha_{\|}=1.09298$ is so close to $\alpha_{\perp}=0.95351$. However, the small shift can actually be detected in experiments.

We believe dielectrophoresis can offer a convenient way of preparing a colloidal crystal [58]. It is not unusual that one fabricates graded colloidal crystals by dielectrophoresis. In this case, the particles in a medium may have different dielectric properties but they must be of the same size (so as to form colloidal crystals). In a nonuniform applied field, the different particles experiences different dielectrophoretic forces according to their strength of polarization. Regarding the fabrication of graded metallic spheres, a practical choice in experiments might possibly be to fabricate multilayered particles with or without dielectric anisotropy [59,60]. In addition, metallic alloying can also be a promising means. In the latter, the dielectric function of the particles no longer obeys the Drude form, and the theoretical calculations are formidable because it involves a Green function formalism for band structure, and a linear response theory (i.e., Kubo formula) for transport properties [61]. In Section 3.1, the objective of considering tetragonal lattices is to achieve anisotropy by changing the lattice parameters, in order to produce a larger optical nonlinearity than in an isotropic system such as a cubic structure.

In summary, we theoretically investigate a class of nonlinear optical materials based on colloidal crystals of graded metallodielectric nanoparticles. Such materials can have both an enhancement and a red shift of optical nonlinearity, due to the gradation inside the metallic core as well as the lattice effects arising from the periodic structure. 


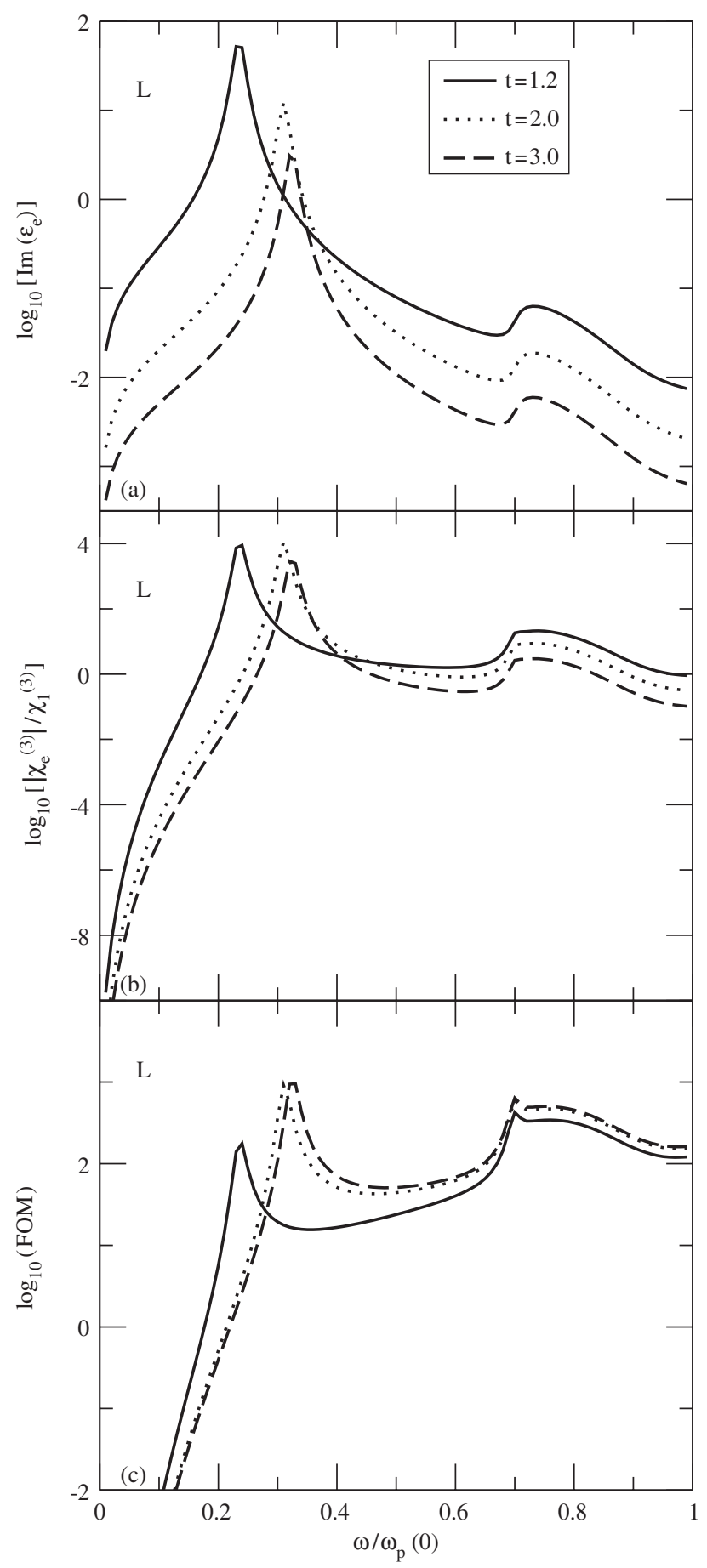

Fig. 9. For the bct lattice, (a) the linear optical absorption $\operatorname{Im}\left(\epsilon_{e}\right)$, (b) the enhancement of the third-order optical nonlinearity $\left|\chi_{e}^{(3)}\right| / \chi_{1}^{(3)}$, and (c) the FOM $\equiv\left|\chi_{e}^{(3)}\right| /\left[\chi_{1}^{(3)} \operatorname{Im}\left(\epsilon_{e}\right)\right]$ versus the normalized incident angular frequency $\omega / \omega_{p}(0)$ for longitudinal field cases (L), for different $t$ [49]. Parameters: $\epsilon_{2}=(3 / 2)^{2}, C_{\omega}=0.3$, and $\gamma=0.02 \omega_{p}(0)$. 


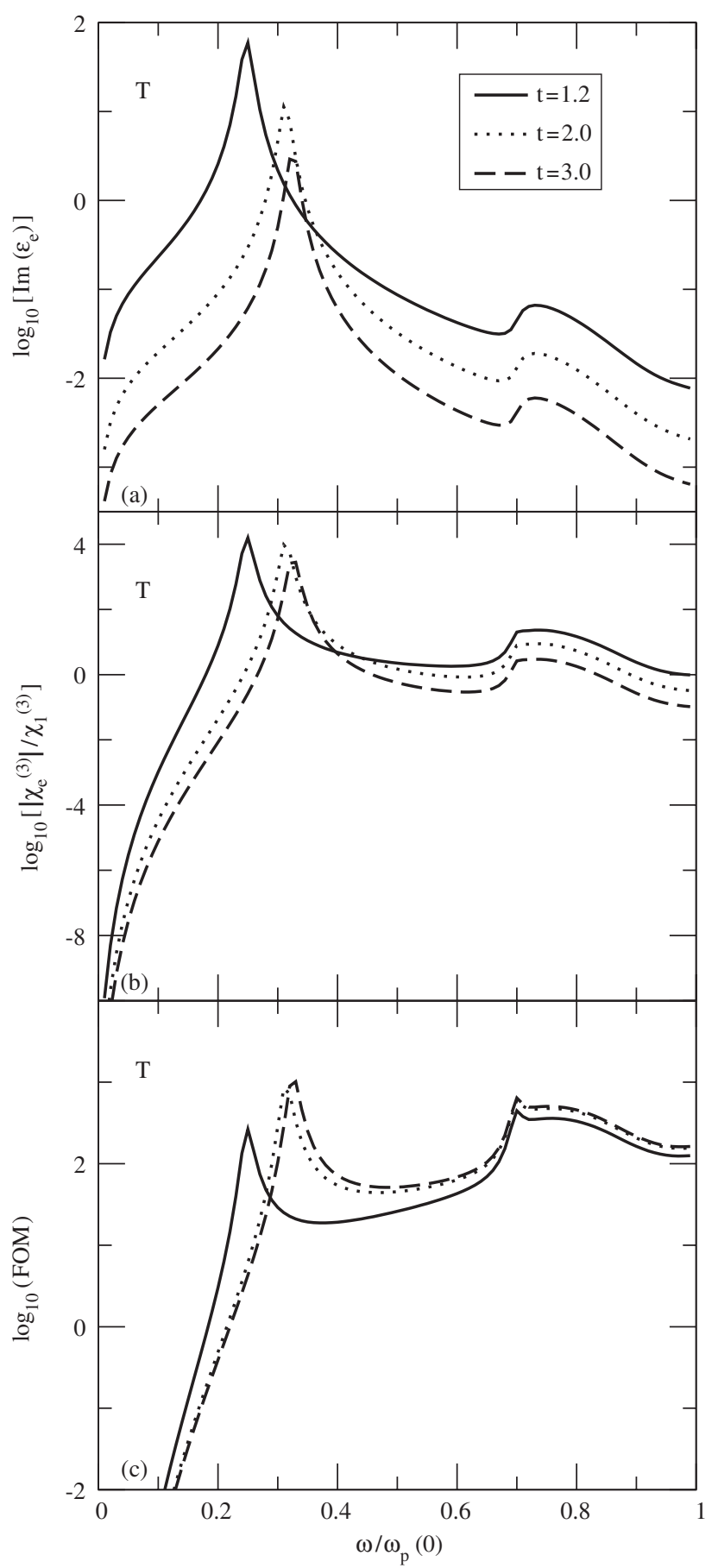

Fig. 10. Same as Fig. 9, but for transverse field cases $(T)$.

\subsection{With a graded-index host}

We shall theoretically investigate a colloidal crystal immersed in a graded-index host and demonstrate a giant enhanced optical nonlinearity band, which is controllable by the gradient and by the easily-tunable colloid structure as 
Table 1

The volume fraction $p$ of the metallic component for bct (i.e., $q=0.87358$ or $\alpha_{\|}=1.09298$ ), bcc (i.e., $q=1.0$ or $\alpha_{\|}=1.0$ ) and fcc (i.e., $q=2^{1 / 3}$ or $\alpha_{\|}=1.0$ ) lattices at various thickness parameter $t$

\begin{tabular}{llll}
\hline & $t=1.2$ & 2.0 & 3.0 \\
\hline bct & $p=0.40401$ & 0.08727 & 0.02586 \\
bcc & $p=0.39362$ & 0.08502 & 0.02519 \\
fcc & $p=0.42852$ & 0.09256 & 0.02743 \\
\hline
\end{tabular}

well. We basically used the quasistatic point-dipole approximation, which suffices in terms of characterizing both the gradient effects and the lattice effects, otherwise the solution is formidable, either from a Green's function formalism or from first-principles [62].

The theoretical calculations are deployed on a model tetragonal lattice with uniaxial anisotropy [see Fig. 11] [52]. Without loss of generality, we will only discuss the bct, bcc and the fcc lattices, respectively. The bcc has the lowest packing density while the fcc has the highest one among the three cases [52]. Extensions to other colloidal structures such as the simple tetragonal lattice are straightforward and similar results are expected. Taking advantages of the interlayer interaction tensor $\mathbf{T}$ (i.e., $T_{i j}$ denotes the interaction strength between two in-plane dipole arrays) given by the Lekner summation method [64-66], we solved the self-consistent equations

$$
\mathbf{E}_{i}=\frac{1}{a^{3}} \sum_{j=1}^{N} N \mathbf{T}_{i j} \cdot\left(\alpha_{j} \mathbf{E}_{j}\right)+\mathbf{E}_{i}^{(0)},
$$

where $a$ is the lattice constant as shown in Fig. $11, \alpha_{j}$ is the layer-dependent linear bare polarizability, here $i, j$ label the crystal layer and $N$ denotes the total layer number. $\mathbf{E}_{i}^{(0)}$ in the self-consistent equation is not simply the externally applied electric field $\mathbf{E}_{0}$ due to the presence of gradient. However, it is the field inside the graded host medium, which is thus determined by virtue of the continuity of the normal component of the electric displacement $\mathbf{D}$ in the longitudinal case, i.e., $\mathbf{E}_{0}$ parallel to the uniaxial axis. It is the $z$-axis as shown in Fig. 11 in our case. Nevertheless in the transverse case ( $\mathbf{E}_{0}$ perpendicular to the uniaxial axis), we exactly used the applied field $\mathbf{E}_{0}$ because the boundary condition now becomes the continuity of the tangential component of electric field. We compared the effective linear and nonlinear optical responses of colloidal crystals with the different lattice structures (i.e., bct, bcc and fcc), made of metallic nanoparticles of linear dielectric constant $\epsilon_{1}$ and third-order nonlinear susceptibility $\chi_{1}$, suspended in a host fluid of $\epsilon_{m}$ [see Fig. 11]. Both the longitudinal (L) and transverse (T) results will be discussed. The self-consistent equations over $i=1,2 \ldots, N$ are then combined together to take into account the lattice effect and is being able to be transformed into a matrix form as

$$
\mathbf{E}=\mathbf{T A E}+\mathbf{E}^{(0)}
$$

More precisely, in the longitudinal and the transverse cases, $\mathbf{E}=\left\{E_{i}^{(L, T)}\right\}$ is simply $N$-dimensional vector and $\mathbf{A}$ is $N \times N$ diagonal matrix of the polarizability, which relates the induced dipole moment of the particle in the layer $\ell_{i}$ and the local field $\mathbf{E}_{i}$, and indeed consists of the linear and nonlinear contributions. That is

$$
\mathbf{p}_{i}=\alpha_{i} \mathbf{E}_{i}+\beta_{i}\left|\mathbf{E}_{i}\right|^{2} \mathbf{E}_{i} / 3,
$$

where $\alpha_{i}=\epsilon_{m} r^{3}\left(\epsilon_{1}-\epsilon_{m}\right) /\left(\epsilon_{1}+2 \epsilon_{m}\right)$. Here $r$ is the radii of the metallic nanoparticles. In view of weak nonlinearity in the colloidal particles with the nonlinear relationship $\mathbf{D}=\epsilon_{1} \mathbf{E}+\chi_{1}|\mathbf{E}|^{2} \mathbf{E}$, by using the perturbation expansion method [67], we obtain

$$
\beta_{i}=\left(\frac{3 \epsilon_{m}}{\epsilon_{1}+2 \epsilon_{m}}\right)^{2}\left|\frac{3 \epsilon_{m}}{\epsilon_{1}+2 \epsilon_{m}}\right|^{2} r^{3} \chi_{1} .
$$

It is noteworthy that the linear local field $\mathbf{E}_{i}$ around the particles in the layer $\ell_{i}$ are actually obtained by assuming no intrinsic nonlinear response, i.e., we set $\chi_{1}=0$ for solving the self-consistent equations, which is appropriate provided 


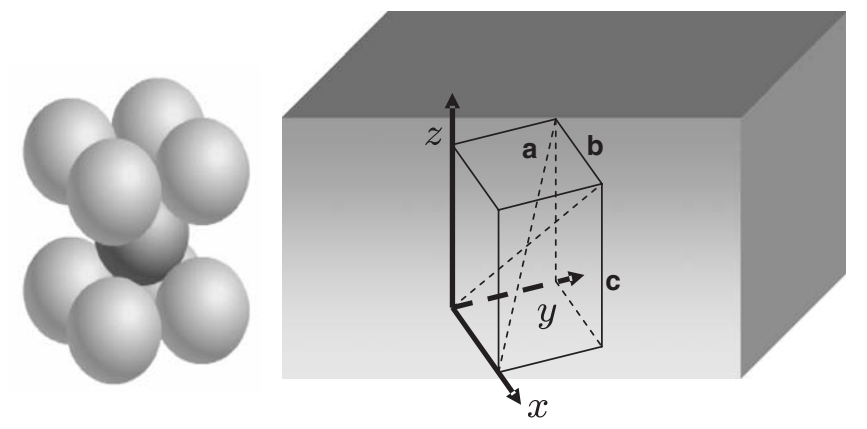

Fig. 11. Schematic of the colloidal crystals immersed in a graded host medium. $a=b$ is assumed in the numerical calculations, which forms square dipolar lattice in $x y$-plane [63].

that the nonlinear responses are much less than the linear ones. Next we use the resultant linear local fields $\mathbf{E}_{i}$ to extract the enhancement factor of the effective nonlinear susceptibility $[67,68]$

$$
\gamma=\frac{\bar{\chi}_{1}}{\chi_{1}}=\rho \frac{\left\langle\left|E_{i}\right|^{2} E_{i}^{2} \beta_{i}\right\rangle}{3 E_{0}^{4} \chi_{1}},
$$

where $\rho=\pi\left[\left(q^{3}+2\right) / q\right]^{3 / 2} / 24$ is the total volume fraction of the metallic colloidal nanoparticles. $1-q$ quantifies the degree of anisotropy of the periodic lattice [52], which also determines the interlayer interaction $\mathbf{T}$, thus results in structure-controllable optical properties. Note that the averaging $\langle\ldots\rangle$ in Eq. (66) is taken over the layers $\ell_{i}(i=1, \ldots, N)$ instead of over the nanoparticles spatial volume, because in this approximation the local fields inside each of the particles are homogeneous. We also assume no nonlinear response in the host, which is in fact relatively neglectable comparing to that in the metal. Additionally, a gradient of the dielectric constant of the host fluid is introduced along the uniaxial direction of the colloidal crystallines, i.e, $\epsilon_{m}=\epsilon_{m}\left(z_{i}\right)$ in our case. In this regard, we treat the host as a continuouslylayered film, thus one explicitly has $\mathbf{E}_{0}=\left\{E_{i}^{(0)}=E_{0} / \epsilon_{m}\left(z_{i}\right)\right\}$ in the longitudinal case. The formation of the gradient in the host might be achieved by dispersing different polymers in it, by selectively filling with microfluidic materials [69], or induced by the presence of a temperature gradient, etc. One can also simply coat the nanoparticles with different coverage shells. But it still remains a challenge because the novel properties from our prediction require a reasonably large gradient in the dielectric constant of the host.

Fig. 12 shows in logarithmic scale the longitudinal optical absorption and the modulus of the nonlinearity enhancement factor $\gamma$ defined in Eq. (66), as functions of the reduced frequency [see the figure caption for more details]. We specifically compared the results of bct $(q=0.87358)$, bcc $(q=1.0)$ and fcc $(q=1.25992)$ as shown in the three columns. The presence of the inhomogeneity in the host fluid obviously leads to a broadened and giant enhanced resonant band in the low-frequency region. This is interesting for potential telecommunication applications. The results of the same colloid suspended in homogeneous host medium with $\epsilon_{m}=1$ (dotted-lines) and $\epsilon_{m}=2.25$ (dashed-lines) are also presented, in an attempt to demonstrate that the broadened resonant band in some sense stems from the hybridization of the non-graded structures. From the absorption spectrum and the enhancement in the third-order nonlinear susceptibility, we would expect an attractive figure of merit [19-23]. That is, the materials effectively exhibit large nonlinearity and relatively small absorption. This is certainly superior to pure metal because it generally has large nonlinearity and unwanted absorption concomitantly. In fact, the optical absorption arises from the surface plasmon resonance, which is obtained from the imaginary part of the effective dielectric constant that is extracted from the generalized Clausius-Mossotti formula [52,62]. Note that the plasmon resonant peaks in the cases of homogeneous host fluid are redshifted with respect to the corresponding ones [not shown] predicted form the Maxwell-Garnett theory. We actually set a gap (e.g., the coated layer thickness in experiments) of $2 r / 5$ between the nearest lattice particles in order to avoid the severe complications arising from the multiple image interactions. It is well known that the multipolar interactions play crucial roles when two particles approach [62]. The introduction of the gap indeed makes the nanoparticles size, and thus the dipole factor relatively small and somewhat unfavorably suppresses the effect arising from variation of the lattice structure, as seen in Fig. 12. 

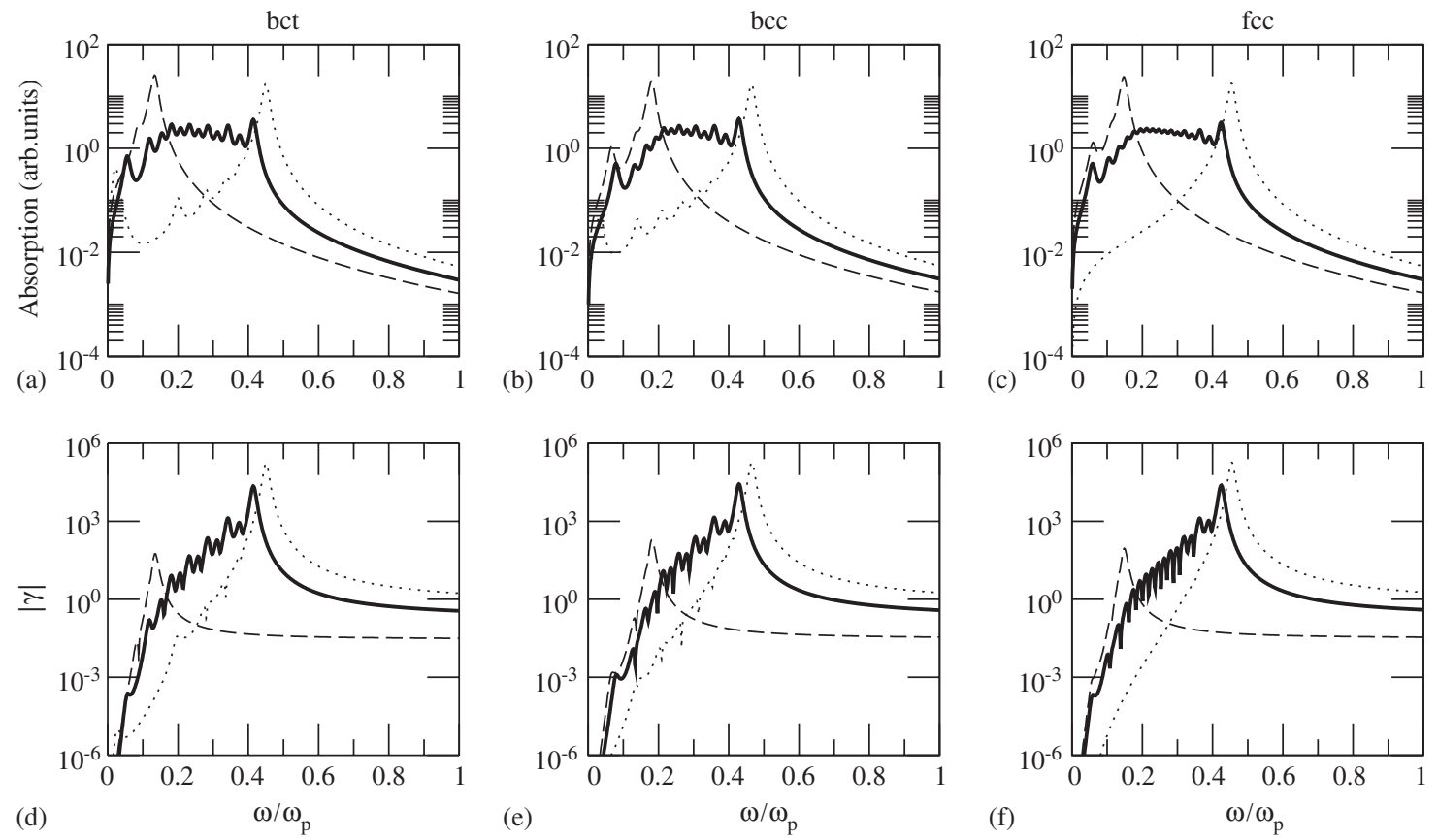

Fig. 12. The effective linear absorption and third-order nonlinearity enhancement factor of the periodic colloid nanoparticles (diameter $a=1$ ) immersed in a graded host fluid whose dielectric constant varies as a function of the layer index $i$ as $\epsilon_{2}(i)=1+1.25 i / N$, and in homogeneous host fluids with dielectric constant $\epsilon_{2}=1.5^{2}$ (dashed) and $\epsilon_{2}=1.0$ (dotted), respectively. The dielectric function of the metallic colloidal nanoparticles are simply denoted by the Drude form $\epsilon_{1}=1-\omega_{p}^{2} /\left(\omega^{2}+\mathrm{i} \omega \Gamma\right)$ [63]. Parameters: $\Gamma=0.02 \omega_{p}, N=25, r=1$, and $E_{0}=1$.

Furthermore, due to the fact that we treated the continuous variations of dielectric function in the host as layered ones in obtaining $\alpha_{i}$ and $\beta_{i}$, and the fact that the dipoles are actually distributed in discrete lattice nodes, a series of sharp peaks are also observable in Fig. 12. The peaks are merged in the broadened band and they become more notable for a increased gradient in the host dielectric constant [not shown], whereas they tend to disappear as the crystal layer $N$ is increased. This is also understandable in the generalized Bergman-Milton spectral representation in graded composites [70]. In detail, the merging is explicitly shown in Fig. 13, where we increased the layer to $N=50$. The fcc is taken as an example and we present both the longitudinal and the transverse results. The peaks in Fig. 12(c) and (f) are distinctly smeared out in Fig. 13(a) and (b), respectively. The transverse results [see Fig. 13(c) and (d)] in the presence of the gradient is slightly different to that in the longitudinal case, but still retain the broadened bands. We ascribe this to the fact that the layer-to-layer interactions fall off exponentially due to the screening effect from the lattice [64-66], therefore give no much layer-structure-dependent interactions for both the two cases. Note that the longitudinal and transverse results of crystals in homogeneous host [dotted and dashed lines in Fig. 13] do not differ much as well.

In conclusion, we have theoretically investigated the optical resonant enhancement due to lattice effect and gradient effect in colloidal crystals, which are made out of suspended metallic nanoparticles in a graded-index host. The gradient in the fluid and the colloid structure are easily subjected to tunability, for example, the structure transformation might be induced by electrorheological effects or by self-assembly of two kinds of particles with biochemically different surface properties, etc.

\section{Metallic films}

Thin films can possess different optical properties (see, e.g., Ref. [71]) when comparing with their bulk counterparts. Recently, some authors found experimentally that the graded thin films may have better dielectric properties than a single-layer film [72]. Graded materials [39] are the materials whose material properties can vary continuously in space. These materials have attracted much interest in various engineering applications [73]. 

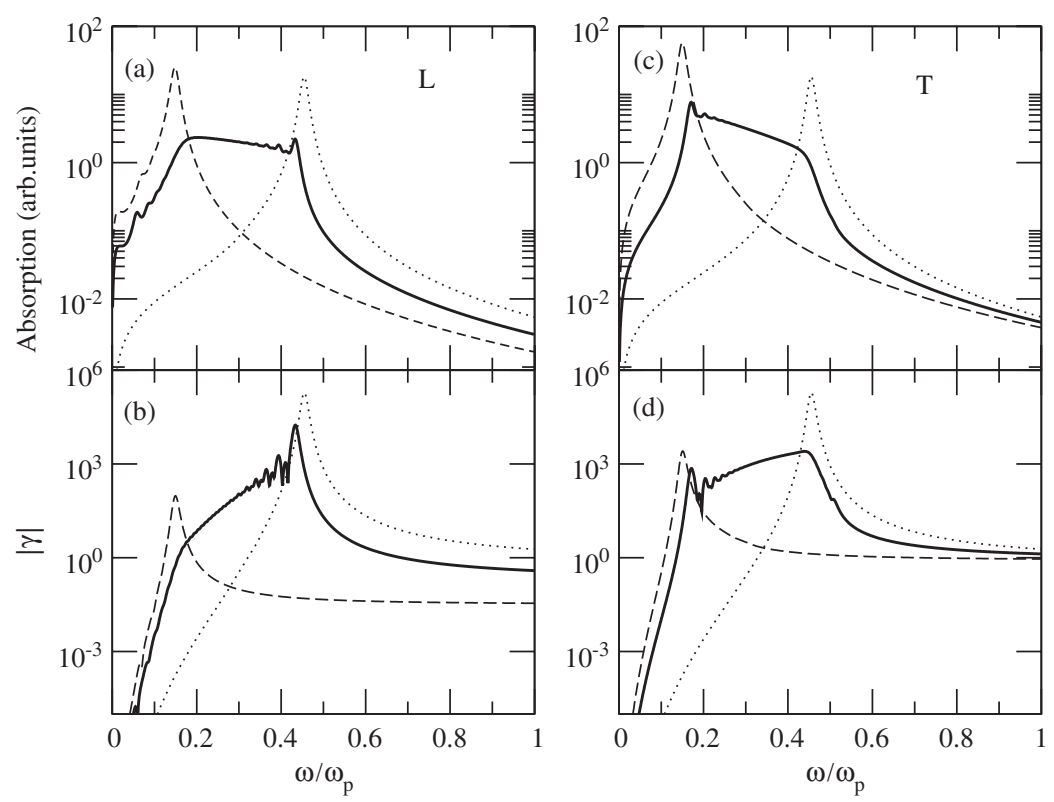

Fig. 13. The same as Fig. 12, but with totally $N=50$ layers in the fcc lattice: (a) and (b) the longitudinal case; (c) and (d) the transverse case [63].

The problem becomes more complicated by the presence of nonlinearity in realistic composites. Besides gradation (inhomogeneity), nonlinearity plays also an important role in the effective material properties of composite media $[3,6,17,36,68,74-79]$. A large nonlinearity enhancement was found indeed when the authors studied a sub-wavelength multilayer of titanium dioxide and conjugated polymer [4].

The surface plasmon resonant nonlinearity enhancement is often accompanied by a strong absorption, and this behavior renders the figure of merit (FOM) of the resonant enhancement peak to be too small to be useful. To circumvent this problem, we proposed to exploit new materials, namely, inhomogeneous (graded) metallic films, in order to achieve a large nonlinearity enhancement and an optimal FOM [21].

As a matter of fact, in practice it is more convenient to fabricate multilayer metallic films than graded films as multilayer metallic films can be readily prepared in a filtered arc deposition system. Therefore, it is necessary to discuss the multilayer effect as the number of layers inside the films increases. In this regard, this should be expected to have practical relevance. As the number of layers $N$ increases, we shall show a gradual transition from sharp peaks to an emerging broad continuous band and the graded film results recover in the limit $N \rightarrow \infty$.

\subsection{Inhomogeneous metallic films}

Let us consider an inhomogeneous (graded) metallic film with width $L$, and the gradation under consideration is in the direction perpendicular to the film. The local constitutive relation between the displacement (D) and the electric field $(\mathbf{E})$ inside the graded layered geometry is given by

$$
\mathbf{D}(z, \omega)=\epsilon(z, \omega) \mathbf{E}(z, \omega)+\chi(z, \omega)|\mathbf{E}(z, \omega)|^{2} \mathbf{E}(z, \omega),
$$

where $\epsilon(z, \omega)$ and $\chi(z, \omega)$ are respectively the linear dielectric constant and third-order nonlinear susceptibility. Note that both $\epsilon(z, \omega)$ and $\chi(z, \omega)$ are gradation profiles as a function of position $r$. Here we assume that the weak nonlinearity condition is satisfied, that is, the contribution of the second term (nonlinear part $\left.\chi(z, \omega)|\mathbf{E}(z, \omega)|^{2}\right)$ in the right-hand side of Eq. (67) is much less than that of the first term (linear part $\epsilon(z, \omega)$ ) [74]. We further restrict our discussion to the quasi-static approximation, under which the whole layered geometry can be regarded as an effective homogeneous one with effective (overall) linear dielectric constant $\bar{\epsilon}(\omega)$ and effective (overall) third-order nonlinear 
susceptibility $\bar{\chi}(\omega)$. To show the definitions of $\bar{\epsilon}(\omega)$ and $\bar{\chi}(\omega)$, we have [74]

$$
\langle\mathbf{D}\rangle=\bar{\epsilon}(\omega) \mathbf{E}_{0}+\bar{\chi}(\omega)\left|\mathbf{E}_{0}\right|^{2} \mathbf{E}_{0},
$$

where $\langle\cdots\rangle$ denotes the spatial average, and $\mathbf{E}_{0}=E_{0} \hat{e}_{z}$ is the applied field along the $z$-axis.

We adopt the graded Drude dielectric profile

$$
\epsilon(z, \omega)=1-\frac{\omega_{p}^{2}(z)}{\omega(\omega+\mathrm{i} \gamma(z))}, \quad 0 \leqslant z \leqslant L .
$$

In Eq. (69), we adopted various plasma-frequency gradation profile

$$
\omega_{p}(z)=\omega_{p}(0)\left(1-C_{\omega} \cdot z / L\right),
$$

and relaxation-rate gradation profile [80]

$$
\gamma(z)=\gamma(\infty)+\frac{C_{\gamma}}{z / L},
$$

where $C_{\omega}$ is a dimensionless constant (gradient). Here $\gamma(\infty)$ denotes the damping coefficient in the corresponding bulk material. $C_{\gamma}$ is a constant (gradient) which is related to the Fermi velocity. A $z$-dependent profile for the plasma frequency and the relaxation time can be achieved experimentally. One possible way may be to impose a temperature profile, because it has been observed that surface-enhanced Raman scattering is sensitive to temperature [81]. Thus one can tune the surface plasmon frequency by imposing an appropriate temperature gradient [82]. A temperature gradient can also be used in materials with a small bandgap or with a profile on dopant concentrations. In this case one can impose a charge carrier concentration profile to a certain extent. This effect, when coupled with materials with a significant intrinsic nonlinear susceptibility, will give us a way to control the effective nonlinear response. For less-conducting materials one can replace the Drude form of the dielectric constants by a Lorentz oscillator form. It may also be possible to fabricate dirty metal films in which the degree of disorder varies in the $z$ direction and hence leads to a relaxation-rate gradation profile.

Due to the simple layered geometry, we can use the equivalent capacitance of series combination to calculate the linear response, i.e., the optical absorption for the metallic film:

$$
\frac{1}{\bar{\epsilon}(\omega)}=\frac{1}{L} \int_{0}^{L} \frac{\mathrm{d} z}{\epsilon(z, \omega)}
$$

The calculation of nonlinear optical response can proceed as follows. We first calculate local electric field $E(z, \omega)$ by the identity

$$
\epsilon(z, \omega) E(z, \omega)=\bar{\epsilon}(\omega) E_{0}
$$

by virtue of the continuity of electric displacement, where $E_{0}$ is the applied field.

In view of the existence of nonlinearity inside the graded film, the effective nonlinear response $\bar{\chi}(\omega)$ can be written as [74]

$$
\bar{\chi}(\omega) \mathbf{E}_{0}^{4}=\left\langle\chi(z, \omega)\left|\mathbf{E}_{\operatorname{lin}}(z)\right|^{2} \mathbf{E}_{\operatorname{lin}}(z)^{2}\right\rangle
$$

where $E_{\text {lin }}$ is the linear local electric field. Next, the effective nonlinear response can be written as an integral over the layer such as

$$
\bar{\chi}(\omega)=\frac{1}{L} \int_{0}^{L} \mathrm{~d} z \chi(z, \omega)\left|\frac{\bar{\epsilon}(\omega)}{\epsilon(z, \omega)}\right|^{2}\left(\frac{\bar{\epsilon}(\omega)}{\epsilon(z, \omega)}\right)^{2} .
$$

For numerical calculations, we set $\chi(z, \omega)$ to be constant $\left(\chi_{1}\right)$, in an attempt to emphasize the enhancement of the optical nonlinearity. Without loss of generality, the layer width $L$ is taken to be 1 .

Fig. 14 displays the optical absorption $\sim \operatorname{Im}[\bar{\epsilon}(\omega)]$, the modulus of the effective third-order optical nonlinearity enhancement $|\bar{\chi}(\omega)| / \chi_{1}$, as well as the FOM (figure of merit) $|\bar{\chi}(\omega)| /\left\{\chi_{1} \operatorname{Im}[\bar{\epsilon}(\omega)]\right\}$ as a function of the incident angular 

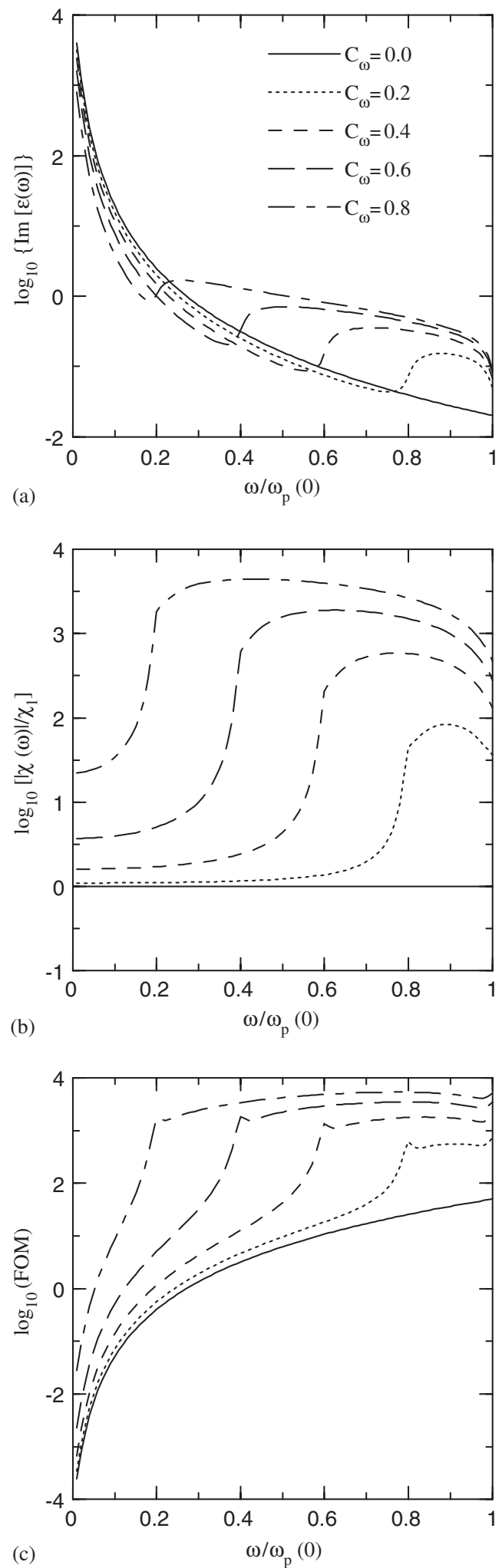

Fig. 14. (a) The linear optical absorption $\operatorname{Im}[\bar{\epsilon}(\omega)]$, (b) the enhancement of the third-order optical nonlinearity $|\bar{\chi}(\omega)| / \chi_{1}$, and (c) the FOM (figure of merit) $\equiv|\bar{\chi}(\omega)| /\left\{\chi_{1} \operatorname{Im}[\bar{\epsilon}(\omega)]\right\}$ versus the normalized incident angular frequency $\omega / \omega_{p}(0)$ for dielectric function gradation profile $\epsilon(z, \omega)=1-\omega_{p}^{2}(z) /[\omega(\omega+\mathrm{i} \gamma(z))]$ with various plasma-frequency gradation profile $\omega_{p}(z)=\omega_{p}(0)\left(1-C_{\omega} \cdot z / L\right)$ and relaxation-rate gradation profile $\gamma(z)=\gamma(\infty)+C_{\gamma} /(z / L)[21]$. Parameters: $\gamma(\infty)=0.02 \omega_{p}(0)$ and $C_{\gamma}=0.0$. 
frequency $\omega$. Here $\operatorname{Im}[\cdots]$ means the imaginary part of $\cdots$. To one's interest, when the positional dependence of $\omega_{p}(z)$ is taken into account (namely, $C_{\omega} \neq 0$ ), a broad resonant plasmon band is observed. As expected, the broad band is caused to appear by the effect of the positional dependence of the plasma frequency of the graded metallic film. In particular, this band can be observed within almost the whole range of frequency, as the gradient $C_{\omega}$ is large enough. In other words, as long as the film under consideration is strongly inhomogeneous, a resonant plasmon band is expected to appear over the whole range of frequency. In addition, it is also shown that increasing $C_{\omega}$ causes the resonant bands to be red-shifted (namely, located at a lower frequency region). In a word, although the enhancement of the effective third-order optical nonlinearity is often accompanied with the appearance of the optical absorption, the FOM is still possible to be quite attractive due to the presence of the gradation of the metallic film.

Similarly, in Fig. 15, we investigate the effect of the inhomogeneity of the relaxation rates $[\gamma(z)]$, which comes from the graded metallic film. It is evident to show that, in the low-frequency region, the positional dependence of relaxation rate $\gamma(z)$ enhances not only the third-order optical nonlinearity but also the FOM of such kind of graded metallic films.

Consequently, graded metallic films can be a suitable candidate material for obtaining the optimal FOM. Thus, corresponding experiments are expected to be done to check our theoretical predictions since graded films can be fabricated easily.

We have discussed a graded metallic film (layered geometry), in an attempt to investigate the effect of gradation on the nonlinear enhancement and FOM of such materials.

In the conventional theory of surface plasmon resonant nonlinearity enhancement, there is often a dielectric component in the system of interest. In this regard, it turns out that it is not difficult to add a homogeneous dielectric layer on the metallic film. The same theory still works but a prominent surface plasmon resonant peak appears at somewhat lower frequencies in addition to the surface plasmon band. Due to the concomitantly strong absorption, the FOM of the resonant enhancement peak is too small to be useful. In the limit of vanishing volume fraction of the dielectric component, however, the present results recover.

Nevertheless, the present results do not depend crucially on the particular form of the dielectric function. The only requirement is that we must have a sufficiently large gradient, either in $\omega_{p}(z)$ or in $\gamma(z)$ to yield a broad plasmon band.

To sum up, we have investigated the effective linear and third-order nonlinear susceptibility of graded metallic films with weak nonlinearity. We calculated the effective linear dielectric constant and third-order nonlinear susceptibility. It has been found that the presence of gradation in metallic films yields a broad resonant plasmon band in the optical region, resulting in a large nonlinearity enhancement and hence an optimal FOM.

\subsection{Multilayer metallic films}

To discuss the multilayer effect on the effective nonlinear optical response, let us first start from a general case, i.e., graded metallic film. In detail, we consider a graded metallic film with width $L$, and its gradation is in the direction perpendicular to the film. As a matter of fact, for graded films, the formalism has been detailedly derived in Ref. [21]. So, below we shall do a brief review, and further add some related backgrounds accordingly.

Inside the graded film, the local constitutive relation between the displacement $\mathbf{D}$ and the electric field $\mathbf{E}$ is given by

$$
\mathbf{D}(z, \omega)=\epsilon(z, \omega) \mathbf{E}(z, \omega)+\chi(z, \omega)|\mathbf{E}(z, \omega)|^{2} \mathbf{E}(z, \omega),
$$

where $\epsilon(z, \omega)$ and $\chi(z, \omega)$ stand for the linear dielectric constant and third-order nonlinear susceptibility, respectively, and both are gradation profiles as a function of position $z$. The weak nonlinearity condition is assumed to be satisfied. That is, the contribution of the second term (i.e., nonlinear part $\left.\chi(z, \omega)|\mathbf{E}(z, \omega)|^{2}\right)$ in the right-hand side of Eq. (75) is much less than that of the first term (namely, linear part $\epsilon(z, \omega)$ ) [74]. In the quasi-static approximation, the whole graded film can be regarded as an effective homogeneous one with effective linear dielectric constant $\bar{\epsilon}(\omega)$ and effective third-order nonlinear susceptibility $\bar{\chi}(\omega)$. Both $\bar{\epsilon}(\omega)$ and $\bar{\chi}(\omega)$ are defined as [74]

$$
\langle\mathbf{D}\rangle=\bar{\epsilon}(\omega) \mathbf{E}_{0}+\bar{\chi}(\omega)\left|\mathbf{E}_{0}\right|^{2} \mathbf{E}_{0},
$$

where $\langle\cdots\rangle$ denotes the spatial average, and $\mathbf{E}_{0}=E_{0} \hat{e}_{z}$ is the applied field along $z$ axis.

Then, we adopt the following graded Drude dielectric profile

$$
\epsilon(z, \omega)=1-\frac{\omega_{p}^{2}(z)}{\omega(\omega+\mathrm{i} \gamma)},
$$



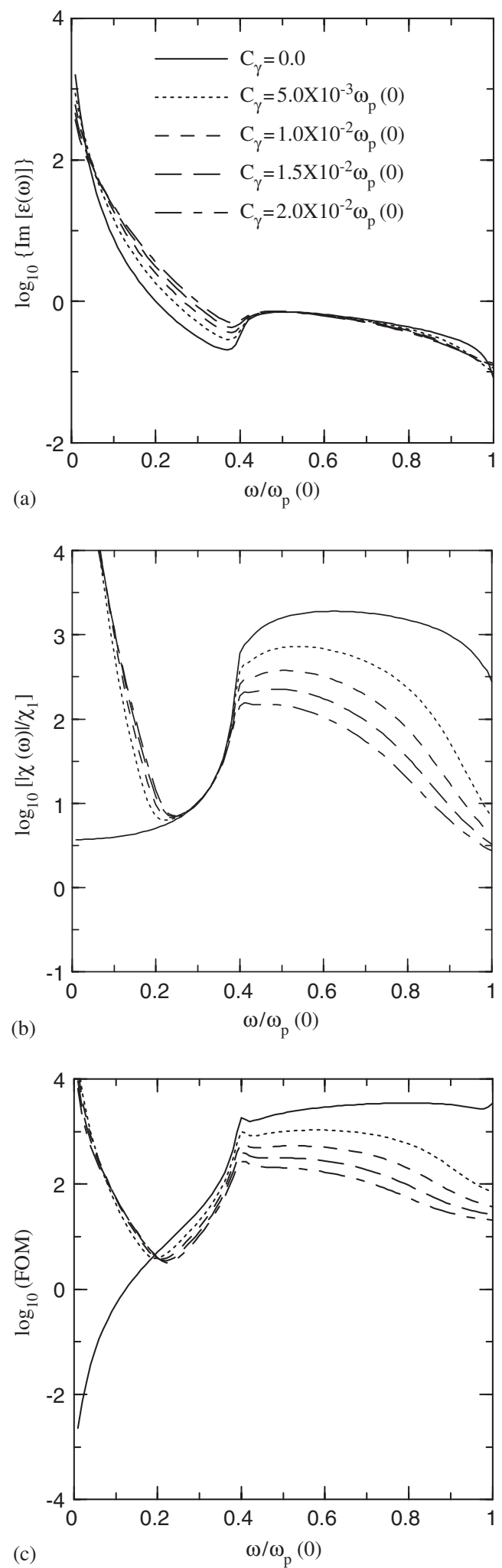

Fig. 15. Same as Fig. 14 [21]. Parameters: $\gamma(\infty)=0.02 \omega_{p}(0)$ and $C_{\omega}=0.6$. 


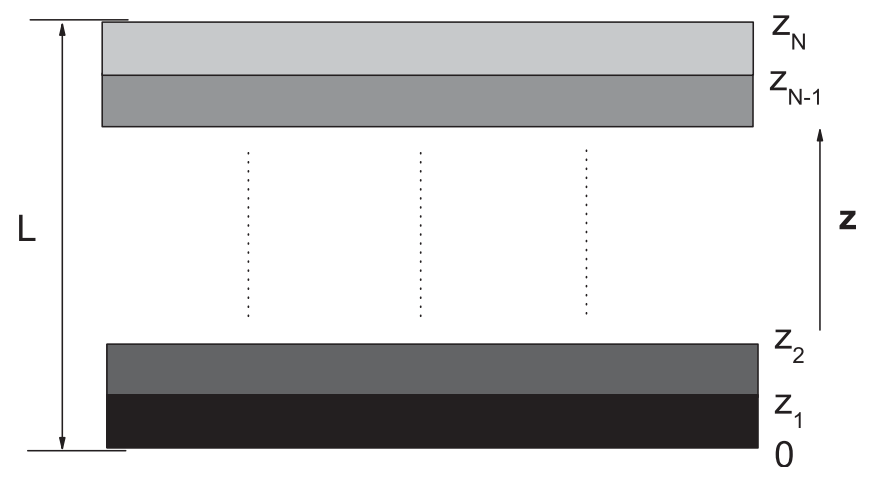

Fig. 16. Schematic graph showing multilayer metallic films.

where $0 \leqslant z \leqslant L$, and $\gamma$ stands for the damping coefficient in the corresponding bulk material. The general form in Eq. (77) allows for the possibility of a gradation profile in the plasma frequency $\omega_{p}(z)$ [e.g., Eq. (70)].

In view of the $z$-dependent profile, let us use the equivalent capacitance for capacitors in series to evaluate the effective perpendicular linear response for a given frequency $\bar{\epsilon}(\omega)$ [21],

$$
\frac{1}{\bar{\epsilon}(\omega)}=\frac{1}{L} \int_{0}^{L} \frac{\mathrm{d} z}{\epsilon(z, \omega)}
$$

Next, we take one step forward to write the effective nonlinear response $\bar{\chi}(\omega)$ as an integral over the film [21],

$$
\bar{\chi}(\omega)=\frac{1}{L} \int_{0}^{L} \mathrm{~d} z \chi(z, \omega)\left|\frac{\bar{\epsilon}(\omega)}{\epsilon(z, \omega)}\right|^{2}\left(\frac{\bar{\epsilon}(\omega)}{\epsilon(z, \omega)}\right)^{2},
$$

where $\chi(z, \omega)$ denotes the local third-order nonlinear susceptibility for a given frequency. It is worth noting that the real $\bar{\chi}(\omega)$ should involve an integral over $x, y$, and $z$ of the local $\chi(x, y, z, \omega)$ multiplied by terms involving $\epsilon(x, y, z, \omega)$. Thus, Eq. (79) offers an approximate $\bar{\chi}(\omega)$, as expected.

To investigate the multilayer effect, we shall use some finite difference approximation of the graded Drude profile [Eq. (77)] for a finite number of layers.

To mimic a multilayer system (Fig. 16), we divide the interval $[0, L]$ into $N$ equally spaced sub-intervals, $\left[0, z_{1}\right]$, $\left(z_{1}, z_{2}\right], \ldots,\left(z_{N-1}, z_{N}\right]$. Then we adopt the midpoint value of $\omega_{p}(z)$ for each sub-interval as the plasma frequency of that sublayer. In this way, we calculate the effective dielectric constant, the effective third-order nonlinear susceptibility, as well as the figure of merit for each $N$. It is worth noting that for $N \rightarrow \infty$ (e.g., $N=1024$ ) the graded film results [21] recover in this limit.

In what follows, we shall do some numerical calculations. We assume that the metal layers within the film have the same real and positive frequency-independent third-order nonlinear susceptibility $\chi(z, \omega)=\chi_{1}$, and do not have a gradation profile. In doing so, we could focus on the enhancement of the optical nonlinearity. Without loss of generality, the film width $L$ is taken to be unity.

For numerical calculations, we take as a model plasma-frequency gradation profile

$$
\omega_{p}(z)=\omega_{p}(0)\left(1-C_{\omega} \cdot z\right),
$$

where $C_{\omega}$ is a constant (gradient) tuning the profile.

Figs. 17-19, respectively, display the optical absorption $\sim \operatorname{Im}[\bar{\epsilon}(\omega)]$, the modulus of the effective third-order optical nonlinearity enhancement $|\bar{\chi}(\omega)| / \chi_{1}$, as well as the $\operatorname{FOM}|\bar{\chi}(\omega)| /\left\{\chi_{1} \operatorname{Im}[\bar{\epsilon}(\omega)]\right\}$ as a function of frequency $\omega / \omega_{p}(0)$. Here $\operatorname{Im}[\cdots]$ means the imaginary part of $\cdots$. In each panel of Figs. 17-19, the corresponding graded film results are shown as well.

It is evident from Figs. 17-19 that for a few layers, say $N=2,4,8$ [(a)-(c)], the optical absorption spectrum and the enhancement of optical nonlinearity consist mainly of sharp peaks. However, the strong optical absorption and the large fluctuation of the nonlinear optical enhancement near these sharp peaks render the FOM too small to be useful. 

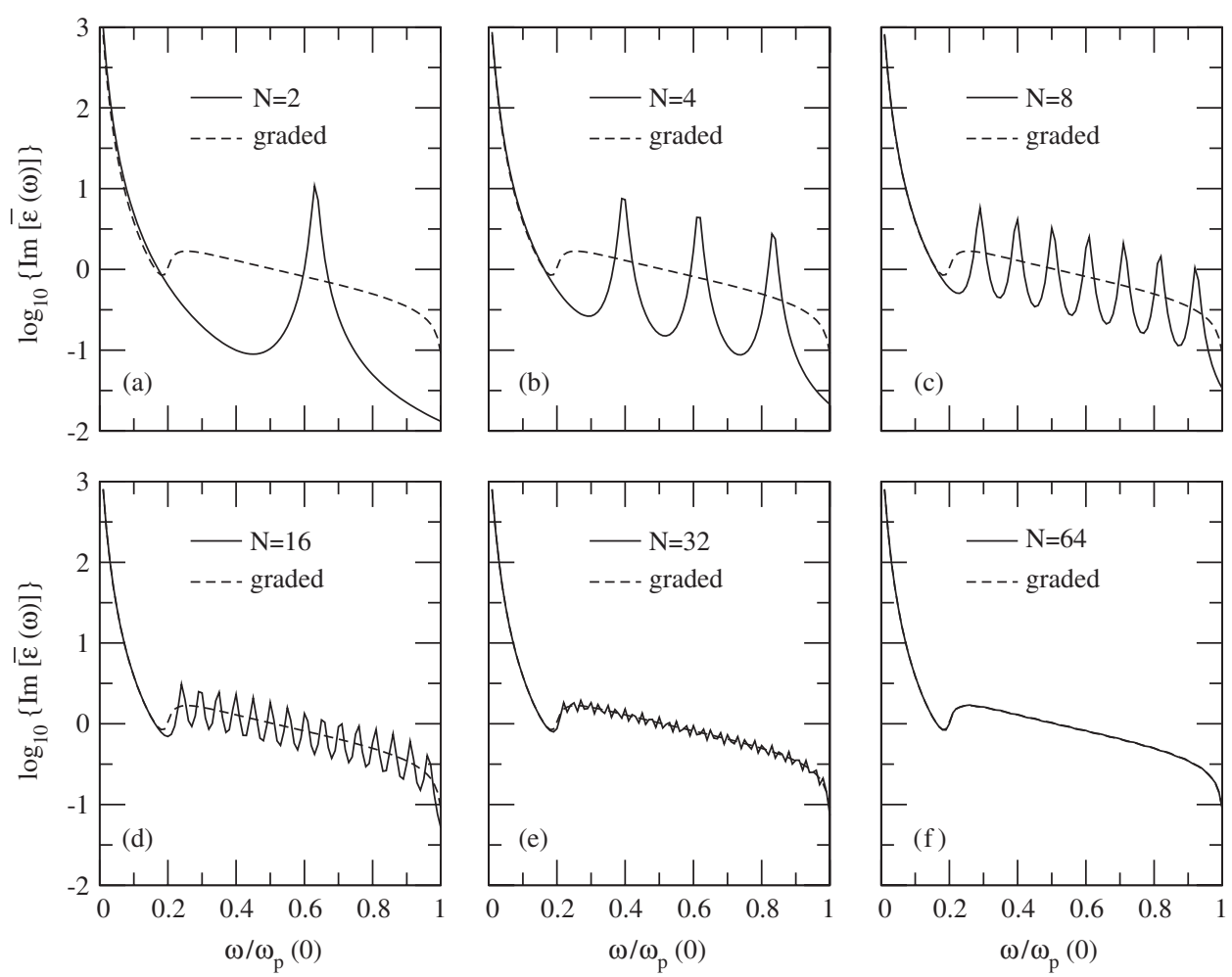

Fig. 17. The linear optical absorption $\operatorname{Im}[\bar{\epsilon}(\omega)]$ versus the normalized incident angular frequency $\omega / \omega_{p}(0)$ for dielectric function gradation profile $\epsilon(z, \omega)=1-\omega_{p}^{2}(z) /[\omega(\omega+\mathrm{i} \gamma)]$ with various plasma-frequency gradation profile $\omega_{p}(z)=\omega_{p}(0)\left(1-C_{\omega} \cdot z\right)$ [83]. Parameters: $\gamma=0.02 \omega_{p}(0)$, $C_{\omega}=0.8, L=1$, and $\chi_{1}=1$.

When the number of layers becomes large [(d)-(f)], the sharp peaks accumulate to a broad band while the fluctuation has been reduced significantly. In this limit, the broad continuous absorption band emerges, and a large FOM persists.

In a word, Figs. 17-19 show a gradual transition from sharp peaks to a broad continuous band as the number of layers increases. This also gives an explanation of the intriguing findings reported in our recent work [21].

To sum up, we have investigated the effective nonlinear optical response of metallic films as the number of layers inside the film increases until the graded film results recover. This is of practical value since in practice it is more convenient to fabricate multilayer metallic films than graded films.

\section{Graded composites}

\subsection{Compositionally graded metal-dielectric composite films of anisotropic particles}

Chemical deposition techniques [Fig. 20(a)] as well as diffusing techniques [Fig. 20(b)] can be used to produce inhomogeneous composite films or interfaces. Metal-dielectric composites have received much attention due to the potential application of their linear and nonlinear optical properties [3,4,6,11,36,68,74-76,79,84-88]. Crucial elements for control of these properties are the micro-structure of the composite, particle shape, and material dispersity. For anisotropically shaped metallic nanoparticles, the resonant plasmon bands split up for orientations along major and minor axes. Furthermore, in case of a large size aspect ratio, the plasmon bands may shift into the infrared, thus enabling the use of metal nanostructures in telecommunication applications in this wavelength range. Compared with spherically shaped particles, anisotropically shaped metallic particles can show reduced plasmon relaxation times [89] as well as enhanced nonlinear responses [90], and may thus be used as building blocks in a variety of optical devices. Some techniques have been developed to fabricate rod-shaped metallic nanoparticles by using lithographic means [91] or 

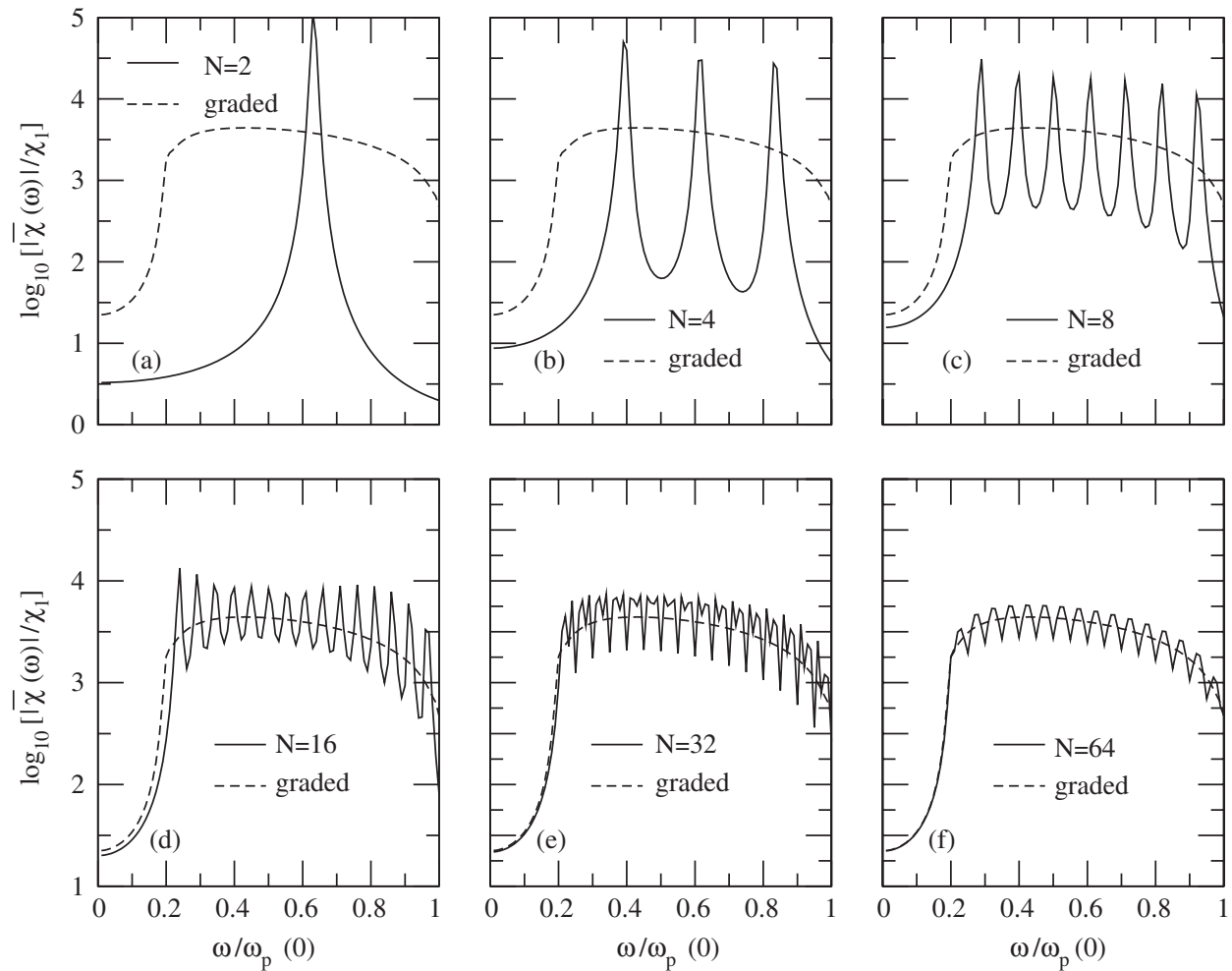

Fig. 18. Same as Fig. 17, but for the enhancement of the third-order optical nonlinearity $|\bar{\chi}(\omega)| / \chi_{1}[83]$.
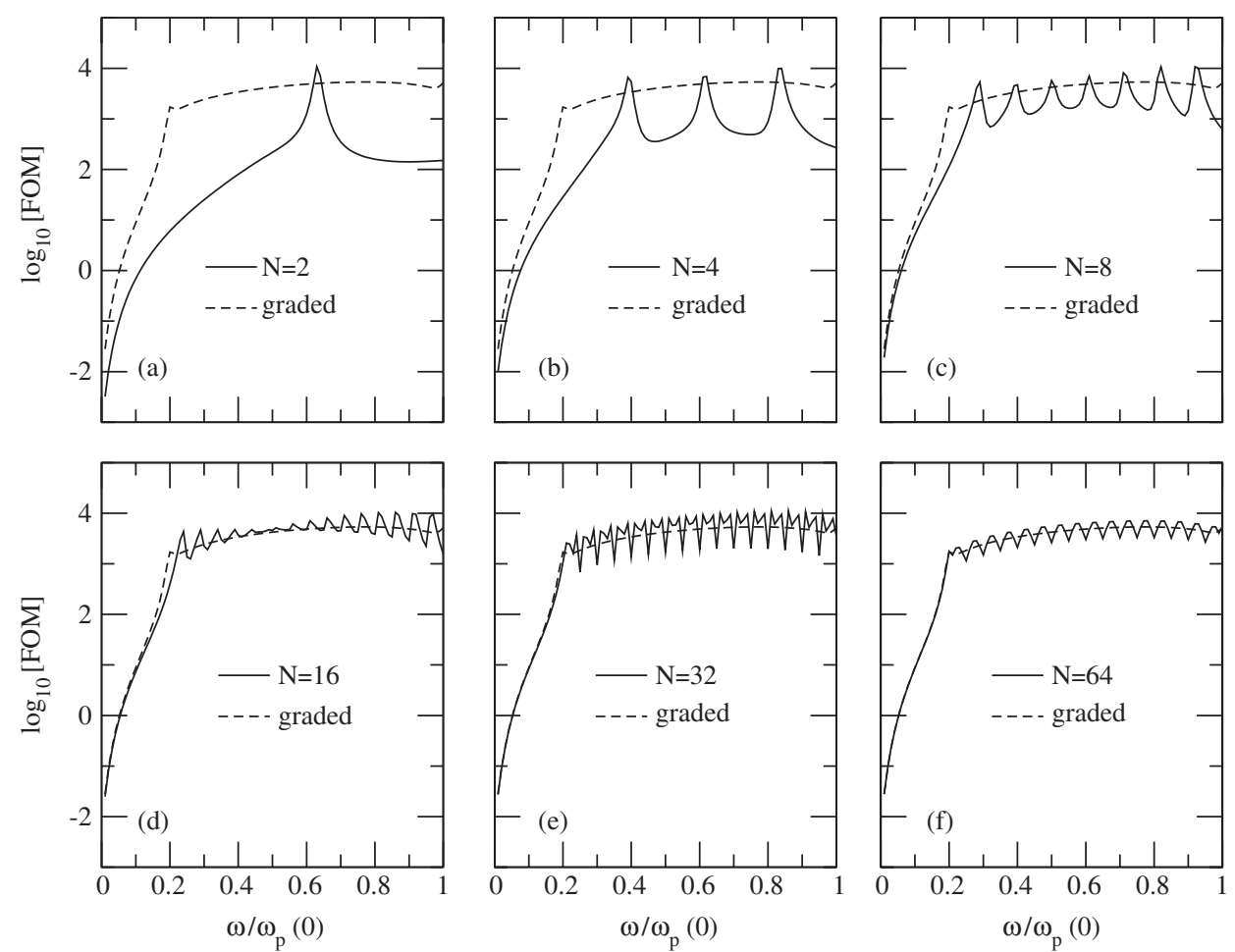

Fig. 19. Same as Fig. 17, but for the $\mathrm{FOM} \equiv|\bar{\chi}(\omega)| /\left\{\chi_{1} \operatorname{Im}[\bar{\epsilon}(\omega)]\right\}[83]$. 


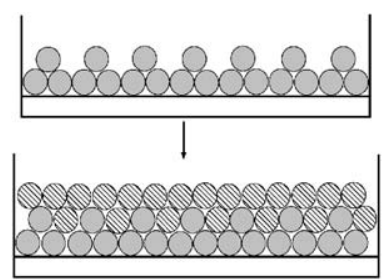

(a)

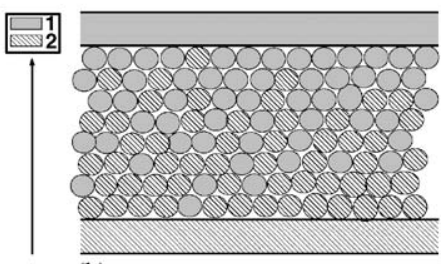

(b)

Fig. 20. Schematic graph showing (a) the chemical deposition technique to form a graded composite film, and (b) the diffusing technique to form a graded interface (composite film) between two bulk composites [94].

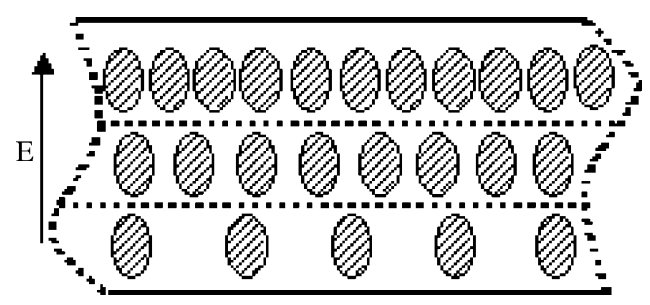

Fig. 21. Schematic graph to show the geometry of a metal-dielectric composite film with a variation of volume fraction of anisotropic particles along $z$ axis perpendicular to the film [99]. The electric field $E$ is parallel to the gradient ( $z$ axis), thus being perpendicular to the film.

anisotropic growth. Recently, one has demonstrated that mega-electron-volt ion irradiation can also be used to modify the shape of nanoparticles [92]. This ion-beam-induced anisotropic deformation effect is known to occur not only for a broad range of amorphous materials [93], but also for crystalline materials including metals [88]. That is, prolate spheroidal metallic particles in a dielectric host can readily be formed by irradiation of mega-electron-volt ions.

The optical property of thin films $[21,23,71,95,96]$ is often different from that of the corresponding bulk material. Recently, a metal-dielectric composite $\left(\mathrm{Au}: \mathrm{BaTiO}_{3}\right)$ film was investigated, and large figure of merit (FOM) was observed [97]. In general, graded materials [39,98] have quite different physical properties from the homogeneous materials. In particular, the graded thin films were found to show better dielectric properties than the single-layer films [72]. A large nonlinearity enhancement was recently found for a sub-wavelength multilayer film of titanium dioxide and conjugated polymer [4]. However, the surface-plasmon resonant nonlinearity enhancement often occurs concomitantly with a strong absorption, and unfortunately this behavior renders the FOM of the resonant enhancement peak to be too small to be useful. To circumvent this problem, we shall consider a kind of graded metal-dielectric composite film, in which a dielectric (or metallic) component is introduced as anisotropically shaped particles embedded in a metallic (or dielectric) component with a compositional and/or shape-dependent gradation profile.

Let us consider a metal-dielectric composite film with a variation of volume fraction of anisotropic particles along $z$ axis perpendicular to the film (Fig. 21). In this case, the local constitutive relation between the displacement field $\mathbf{D}(\mathbf{r}, \omega)$ and electric field $\mathbf{E}(\mathbf{r}, \omega)$ is given by

$$
\mathbf{D}(\mathbf{r}, \omega)=\epsilon(\mathbf{r}, \omega) \mathbf{E}(\mathbf{r}, \omega)+\chi(\mathbf{r}, \omega)|\mathbf{E}(\mathbf{r}, \omega)|^{2} \mathbf{E}(\mathbf{r}, \omega),
$$

where $\epsilon(\mathbf{r}, \omega)$ and $\chi(\mathbf{r}, \omega)$ are the linear dielectric constant and third-order nonlinear susceptibility of a layer inside the graded film, respectively. Here both $\epsilon(\mathbf{r}, \omega)$ and $\chi(\mathbf{r}, \omega)$ are the gradation profiles as a function of position $\mathbf{r}$ and field frequency $\omega$.

The weak nonlinearity condition is assumed to be satisfied. That is, the contribution of the second term [nonlinear part $\left.\chi(\mathbf{r}, \omega)|\mathbf{E}(\mathbf{r}, \omega)|^{2}\right]$ in the right-hand side of Eq. (81) is much less than that of the first term [linear part $\left.\epsilon(\mathbf{r}, \omega)\right]$ [74]. Next, we turn to the quasi-static approximation, under which the whole graded structure can be regarded as an effective homogeneous one with effective linear dielectric constant $\bar{\epsilon}(\omega)$ and effective third-order nonlinear susceptibility $\bar{\chi}(\omega)$. In this connection, $\bar{\epsilon}(\omega)$ and $\bar{\chi}(\omega)$ are defined as [74]

$$
\mathbf{D}_{0}=\bar{\epsilon}(\omega) \mathbf{E}_{0}+\bar{\chi}(\omega)\left|\mathbf{E}_{0}\right|^{2} \mathbf{E}_{0},
$$


where $\mathbf{D}_{0}$ and $\mathbf{E}_{0}\left(=E_{0} \hat{\mathbf{e}}_{z}\right)$ are, respectively, the volume-averaged displacement field and electric field within the whole graded composite film.

For calculating the nonlinear optical response, we shall apply a two-step solution. In Step A, we first derive the responses of a layer inside the graded film, $\bar{\epsilon}(z, \omega)$ and $\bar{\chi}(z, \omega)$. In Step B, the overall responses of the graded film, $\bar{\epsilon}(\omega)$ and $\bar{\chi}(\omega)$, are derived. In the two-step solution, it should be remarked that the local field inside the spheroidal particles is uniform, and the effective nonlinear response of a layer is therefore exact within the Maxwell-Garnett theory. When we have a nonlinear host, we shall have to invoke the decoupling approximation [100]. It is worth noting that Step B is exact, see Eqs. (96)-(98) below. In a word, in Step A, we homogenize the composite film along $x y$ plane while in Step B, we further homogenize the graded film along $z$ axis.

\subsubsection{Responses of a layer inside the graded film: $\bar{\epsilon}(z, \omega)$ and $\bar{\chi}(z, \omega)$}

It is not possible to calculate $\bar{\epsilon}(z, \omega)$ exactly in terms of the compositional and/or shape-dependent gradation profile. Nevertheless, to obtain an estimate of $\bar{\epsilon}(z, \omega)$, we can take a small volume element inside the layer, at a position $z$. Further, this small volume element can be seen as a composite where the locations of the inclusion particles are random in the host medium. This, however, is a highly directional distribution since the long or short axis of prolate or oblate particles is parallel to the gradient along $z$ axis. Accordingly, the volume fraction of the inclusion is $p(z)$ for the dielectric or $1-p(z)$ for the metal. In this regard, the well-known Maxwell-Garnett approximation holds very well for computing $\bar{\epsilon}(z, \omega)$ [as shown in Eqs. (83) and (84) below]. In detail, for the dielectric particles embedded in the metallic component in a layer, $\bar{\epsilon}(z, \omega)$ can be given by the first-kind of Maxwell-Garnett approximation (MGA1) $[27,28,101]$

$$
\frac{\bar{\epsilon}(z, \omega)-\epsilon_{1}(\omega)}{L_{z}^{(2)} \bar{\epsilon}(z, \omega)+\left(1-L_{z}^{(2)}\right) \epsilon_{1}(\omega)}=p(z) \frac{\epsilon_{2}-\epsilon_{1}(\omega)}{L_{z}^{(2)} \epsilon_{2}+\left(1-L_{z}^{(2)}\right) \epsilon_{1}(\omega)},
$$

where $L_{z}^{(2)}$ is the depolarization factor of the dielectric particles along $z$-axis, and satisfies a sum rule $L_{z}^{(2)}+2 L_{x}^{(2)}=1$. Here $L_{x}^{(2)}$ is the depolarization factor of the dielectric particles along $x(y)$-axis, $\epsilon_{2}\left(\right.$ or $\left.\epsilon_{1}(\omega)\right)$ stands for the dielectric constant of the dielectric (or metallic) particles, and $p(z)$ denotes the volume fraction of the dielectric particles in each layer which is thus a compositional gradation profile as a function of position $z$. Alternatively, for the metallic particles embedded in the dielectric host, the second kind of Maxwell-Garnett approximation (MGA2) can be used to determine $\bar{\epsilon}(z, \omega)$, such that $[27,28,101]$

$$
\frac{\bar{\epsilon}(z, \omega)-\epsilon_{2}}{L_{z}^{(1)} \bar{\epsilon}(z, \omega)+\left(1-L_{z}^{(1)}\right) \epsilon_{2}}=(1-p(z)) \frac{\epsilon_{1}(\omega)-\epsilon_{2}}{L_{z}^{(1)} \epsilon_{1}(\omega)+\left(1-L_{z}^{(1)}\right) \epsilon_{2}},
$$

where $L_{z}^{(1)}$ is the depolarization factor of the metallic particles along $z$-axis. Similarly, there exists $L_{z}^{(1)}+2 L_{x}^{(1)}=1$ where $L_{x}^{(1)}$ is the depolarization factor of the metallic particles along $x(y)$-axis. It is worth noting that $L_{z}<1 / 3,=1 / 3$ and $>1 / 3$ indicates the fact that the metallic (or dielectric) particles exist in the form of prolate spheroid, sphere and oblate spheroid, respectively. In Eqs. (83) and (84), the dielectric constant of the metal $\epsilon_{1}(\omega)$ is given by the known Drude expression

$$
\epsilon_{1}(\omega)=1-\frac{\omega_{p}^{2}}{\omega(\omega+\mathrm{i} \gamma)},
$$

where $\omega_{p}$ denotes the bulk plasmon frequency, and $\gamma$ the collision frequency. In case of $\epsilon_{1}(\omega)>\epsilon_{2}$, the MGA1 always gives an upper bound while the MGA2 a lower bound, and vice versa. The exact result must lie between the two bounds [102]. For both the MGA1 and MGA2, the particles under discussion are randomly embedded but their orientations are all along $z$ axis (i.e., perpendicular to the film). The reason is that in experiments the prolate spheroidal metallic particles can be highly oriented along the direction of irradiated ions [88]. For completeness, we shall also numerically calculate the case of oblate spheroids.

Then, we calculate the effective nonlinear response for a layer at position $z, \bar{\chi}(z, \omega)$ [74],

$$
\bar{\chi}(z, \omega) \mathbf{E}(z)^{4}=(1-p(z)) \chi_{1}\left\langle\left|\mathbf{E}_{1}(z)\right|^{2} \mathbf{E}_{1}(z)^{2}\right\rangle+p(z) \chi_{2}\left\langle\left|\mathbf{E}_{2}(z)\right|^{2} \mathbf{E}_{2}(z)^{2}\right\rangle
$$

where $\chi_{1}$ and $\chi_{2}$ are, respectively, the (intrinsic) third-order nonlinear susceptibility of the metallic and dielectric components, $E_{1}(z)$ (or $E_{2}(z)$ ) represents the local electric field inside the metallic (or dielectric) component within 
a layer at position $z, E(z)$ denotes the volume-averaged electric field within the layer, and $\langle\cdots\rangle$ stands for the volume average of $\cdots$ within the layer. In order to estimate $\bar{\chi}(z, \omega)$, due to the existence of nonlinear host we have to invoke the decoupling approximation [100]

$$
\left\langle\left|\mathbf{E}_{i}(z)\right|^{2} \mathbf{E}_{i}(z)^{2}\right\rangle=\left\langle\left|\mathbf{E}_{i}(z)\right|^{2}\right\rangle\left\langle\mathbf{E}_{i}(z)^{2}\right\rangle, \quad i=1,2 .
$$

For the sake of consistency, the local field averages $\left\langle\left|\mathbf{E}_{i}(z)\right|^{2}\right\rangle$ and $\left\langle\mathbf{E}_{i}(z)^{2}\right\rangle$ should be determined by using the Maxwell-Garnett technique [103]. For the MGA1, we obtain the local field averages such that

$$
\begin{aligned}
\left\langle\mathbf{E}_{2}(z)^{2}\right\rangle & =\frac{\left(L_{z}^{(2)}\right)^{-2} \epsilon_{1}(\omega)^{2}}{\left[(1-p(z)) \epsilon_{2}+\left(\left(L_{z}^{(2)}\right)^{-1}-(1-p(z))\right) \epsilon_{1}(\omega)\right]^{2}} \mathbf{E}(z)^{2}, \\
\left\langle\mathbf{E}_{1}(z)^{2}\right\rangle & =\theta\left(1-\frac{p(z)\left(L_{z}^{(2)}\right)^{-1}\left[\left(L_{z}^{(2)}\right)^{-1} \epsilon_{1}(\omega)^{2}-(1-p(z))\left(\epsilon_{2}-\epsilon_{1}(\omega)\right)^{2}\right]}{\left[(1-p(z)) \epsilon_{2}+\left(\left(L_{z}^{(2)}\right)^{-1}-(1-p(z))\right) \epsilon_{1}(\omega)\right]^{2}}\right) \mathbf{E}(z)^{2}, \\
\left\langle\left|\mathbf{E}_{2}(z)\right|^{2}\right\rangle & =\frac{\left(L_{z}^{(2)}\right)^{-2}\left|\epsilon_{1}(\omega)\right|^{2}}{\left|(1-p(z)) \epsilon_{2}+\left(\left(L_{z}^{(2)}\right)^{-1}-(1-p(z))\right) \epsilon_{1}(\omega)\right|^{2}} \mathbf{E}(z)^{2}, \\
\left\langle\left|\mathbf{E}_{1}(z)\right|^{2}\right\rangle & =\theta\left(1-\frac{p(z)\left(L_{z}^{(2)}\right)^{-1}\left[\left(L_{z}^{(2)}\right)^{-1}\left|\epsilon_{1}(\omega)\right|^{2}-(1-p(z))\left|\epsilon_{2}-\epsilon_{1}(\omega)\right|^{2}\right]}{\left|(1-p(z)) \epsilon_{2}+\left(\left(L_{z}^{(2)}\right)^{-1}-(1-p(z))\right) \epsilon_{1}(\omega)\right|^{2}}\right) \mathbf{E}(z)^{2},
\end{aligned}
$$

with $\theta=1 /(1-p(z))$. Similarly, for the MGA2, the local field averages are given by

$$
\begin{aligned}
\left\langle\mathbf{E}_{1}(z)^{2}\right\rangle & =\frac{\left(L_{z}^{(1)}\right)^{-2} \epsilon_{2}^{2}}{\left[p(z) \epsilon_{1}(\omega)+\left(\left(L_{z}^{(1)}\right)^{-1}-p(z)\right) \epsilon_{2}\right]^{2}} \mathbf{E}(z)^{2}, \\
\left\langle\mathbf{E}_{2}(z)^{2}\right\rangle & =\theta^{\prime}\left(1-\frac{(1-p(z))\left(L_{z}^{(1)}\right)^{-1}\left[\left(L_{z}^{(1)}\right)^{-1} \epsilon_{2}^{2}-p(z)\left(\epsilon_{1}(\omega)-\epsilon_{2}\right)^{2}\right]}{\left[p(z) \epsilon_{1}(\omega)+\left(\left(L_{z}^{(1)}\right)^{-1}-p(z)\right) \epsilon_{2}\right]^{2}}\right) \mathbf{E}(z)^{2}, \\
\left\langle\left|\mathbf{E}_{1}(z)\right|^{2}\right\rangle & =\frac{\left(L_{z}^{(1)}\right)^{-2}\left|\epsilon_{2}\right|^{2}}{\left|p(z) \epsilon_{1}(\omega)+\left(\left(L_{z}^{(1)}\right)^{-1}-p(z)\right) \epsilon_{2}\right|^{2}} \mathbf{E}(z)^{2}, \\
\left\langle\left|\mathbf{E}_{2}(z)\right|^{2}\right\rangle & =\theta^{\prime}\left(1-\frac{(1-p(z))\left(L_{z}^{(1)}\right)^{-1}\left[\left(L_{z}^{(1)}\right)^{-1}\left|\epsilon_{2}\right|^{2}-p(z)\left|\epsilon_{1}(\omega)-\epsilon_{2}\right|^{2}\right]}{\left|p(z) \epsilon_{1}(\omega)+\left(\left(L_{z}^{(1)}\right)^{-1}-p(z)\right) \epsilon_{2}\right|^{2}}\right) \mathbf{E}(z)^{2},
\end{aligned}
$$

with $\theta^{\prime}=1 / p(z)$.

\subsubsection{Overall responses of the graded film: $\bar{\epsilon}(\omega)$ and $\bar{\chi}(\omega)$}

Owing to the simple graded structure (Fig. 21), we can use the equivalent capacitance of series combination to calculate the linear response (i.e., optical absorption for the graded film), $\bar{\epsilon}(\omega)$,

$$
\frac{1}{\bar{\epsilon}(\omega)}=\frac{1}{W} \int_{0}^{W} \frac{\mathrm{d} z}{\bar{\epsilon}(z, \omega)}
$$

where $W$ is the thickness of the film.

By virtue of the continuity of electric displacement, there is a relation

$$
\bar{\epsilon}(z, \omega) E(z)=\bar{\epsilon}(\omega) E_{0} .
$$

Then, we take one step forward to obtain the effective third-order nonlinear susceptibility $\bar{\chi}(\omega)$ as an integral over the graded film,

$$
\bar{\chi}(\omega)=\frac{1}{W} \int_{0}^{W} \mathrm{~d} z \bar{\chi}(z, \omega)\left|\frac{\bar{\epsilon}(\omega)}{\bar{\epsilon}(z, \omega)}\right|^{2}\left(\frac{\bar{\epsilon}(\omega)}{\bar{\epsilon}(z, \omega)}\right)^{2} .
$$


In what follows, we shall do some numerical calculations. Set both $\chi_{1}$ and $\chi_{2}$ to be a real and positive frequencyindependent constant $\chi_{0}$, so that we could emphasize the enhancement of the optical nonlinearity. Without loss of generality, the film thickness $W$ is taken to be unity. For the model calculation, we shall use the gradation profile

$$
p(z)=a z^{m},
$$

where $a$ and $m$ are constants tuning the profile.

Fig. 22 shows the results obtained from the MGA1 [Eq. (83)]. In this figure, we display: (a) the optical absorption $\sim \operatorname{Im}[\bar{\epsilon}(\omega)]$; (b) the modulus of the effective third-order optical nonlinearity enhancement $|\bar{\chi}(\omega)| / \chi_{0}$; and (c) the FOM $|\bar{\chi}(\omega)| /\left\{\chi_{0} \operatorname{Im}[\bar{\epsilon}(\omega)]\right\}$ as a function of the incident angular frequency $\omega$, for different $L_{z}^{(2)}$. Here $\operatorname{Im}[\cdots]$ means the imaginary part of $\cdots$. When the layer gradation profile $p(z)=a z^{m}$ is taken into account, a broad resonant plasmon band is observed for any $L_{z}^{(2)}$ of interest. In other words, the broad band is caused to appear by the effect of the positional dependence of the dielectric or metallic component. This conclusion may be made by comparing the curves in Fig. 22 with those of $n=0$ (corresponding to the case where the effects of gradation as well as non-spherical shape are excluded) in Fig. 27. Moreover, we find that decreasing $L_{z}^{(2)}$ makes the resonant bands in both optical nonlinearity and optical absorption broader. Although the enhancement of the effective third-order optical nonlinearity is often accompanied with the appearance of the optical absorption, the FOM is still possible to be very attractive [see Fig. 22(c)] due to the positional dependence of the dielectric or metallic components. In particular, the particle shape can also be used to enhance the FOM significantly. It is worth noting that there is a prominent surface plasmon resonant peak which appears at somewhat higher frequencies in addition to the surface plasmon band at lower frequencies.

Fig. 23 displays the results which were obtained from the MGA2 [Eq. (84)]. For this figure, we see the parallel shaped metallic particles are randomly embedded in the dielectric host in each layer. In contrast, the surface plasmon resonant peak is found to locate at lower frequencies in addition to the surface plasmon band which locates at higher frequencies. Also, it is shown that the broad plasmon bands in optical nonlinearity and absorption is caused to appear by the effect of gradation, when comparing the curves in Fig. 23 with those of $n=0$ in Fig. 28. Note the latter corresponds to the case without the effects of gradation and non-spherical shape. Decreasing $L_{z}^{(1)}$ causes the plasmon bands to be broadened. This effect makes the FOM can be very attractive.

The MGA1 was applied to plot Fig. 24, in an attempt to discuss the effect of the gradation of the volume fraction of the dielectric by means of the gradation profile $p(z)=a z^{m}$ for different $(\mathrm{a}-\mathrm{c}) a$ and $(\mathrm{d}-\mathrm{f}) m$. In other words, we investigate a compositional gradation profile in the film, in which the dielectric particles possess a positional-dependent volume fraction. In detail, increasing $a$ causes the resonant plasmon bands in optical nonlinearity and absorption to be broadened, see Fig. 24(a-b). Accordingly, in case of gradation, the FOM can be more attractive, see Fig. 24(c). Similarly, Fig. 24(d-f) displays the influence of $m$. It is apparent to see that the broad resonant plasmon bands in optical nonlinearity and absorption can be enhanced by increasing $m$ [see Fig. 24(d-e)]. Finally, the FOM can become more attractive in the frequency range $0.3 \omega_{p}<\omega<0.7 \omega_{p}$, as $m$ increases, see Fig. 24(f).

Similar to Fig. 24, Fig. 25 is plotted via the MGA2. First, we discuss the effect of $a$. In both optical nonlinearity and absorption, the resonant plasmon bands are caused to be both enhanced and broadened by increasing $a$, as yielding a very attractive FOM in Fig. 25(c). On the other hand, the $m$ effect on the optical nonlinearity and FOM plays a role too [see Fig. 25(e-f)], and accordingly both the optical nonlinearity and FOM can be enhanced accordingly. In addition, as $a$ or $m$ varies, the plasmon resonant peaks in Fig. 25 have the same red-shift (located at lower frequency) or blue-shift (located at higher frequency) behavior as those shown in Fig. 24 where the MGA1 was used instead.

During ion irradiation, the ion energy can be much larger at the top of the film than that at the bottom. Therefore, the particles can be much prolate at the top, but they are relatively spherical at the bottom. In other words, both $L_{z}^{(1)}$ and $L_{z}^{(2)}$ can be small at the top of the film, while increases to roughly $1 / 3$ at the bottom of the film. In this regard, we could introduce a gradation in the depolarization factor (Fig. 26) rather than in the volume fraction. Namely, in this case, $L_{z}(z)$ is a function of $z$ (Fig. 26). For convenience, we keep the volume fraction to be constant [e.g., $p(z)=0.85$ ] for each layer throughout the film, and take a physical profile $L_{z}(z)=(1 / 3) z^{n}$. In particular, as $n=0$, we have $L_{z}(z)=1 / 3$, i.e., the gradation in the depolarization factor and the non-spherical shape effect disappear. For different $n$, the corresponding results are shown in Figs. 27 and 28 for the MGA1 and MGA2, respectively. It is shown that the $L_{z}(z)$ profile does have a significant impact on the optical response, as expected. In Fig. 27(a), the plasmon peak shows a reduction as well as a blue-shift as $n$ changes from zero (without gradation) to nonzero (with gradation), and accordingly the optical nonlinearity and hence the FOM is reduced. The difference between the results for different 

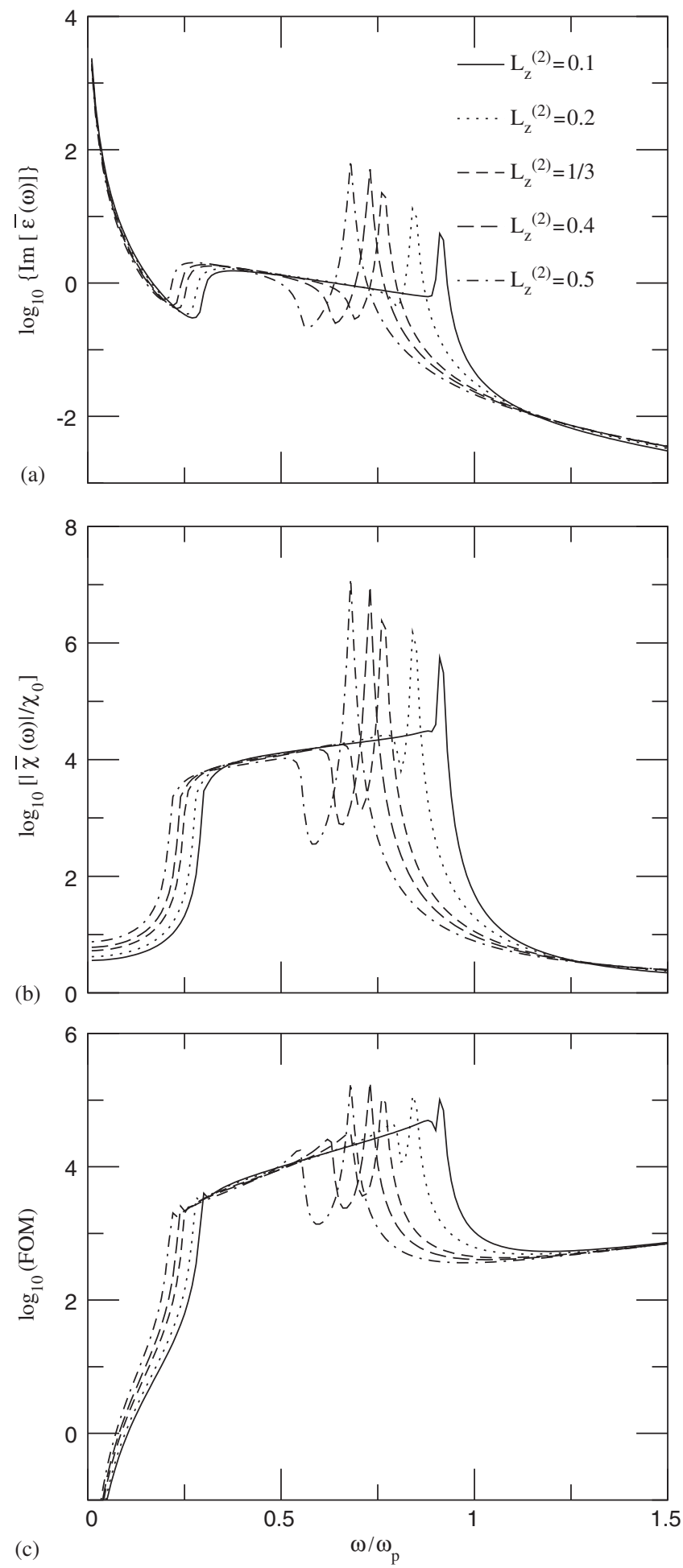

Fig. 22. Results for the MGA1 [Eq. (83)] [99]: (a) Linear optical absorption $\operatorname{Im}[\bar{\epsilon}(\omega)]$; (b) enhancement of the third-order optical nonlinearity $|\bar{\chi}(\omega)| / \chi_{0}$; and (c) FOM $\equiv|\bar{\chi}(\omega)| /\left\{\chi_{0} \operatorname{Im}[\bar{\epsilon}(\omega)]\right\}$ versus the normalized incident angular frequency $\omega / \omega_{p}$ for layer dielectric profile $p(z)=a z^{m}$, for different $L_{z}^{(2)}$. Parameters: $a=0.8, m=1, \gamma / \omega_{p}=0.01$, and $\epsilon_{2}=(3 / 2)^{2}$. 

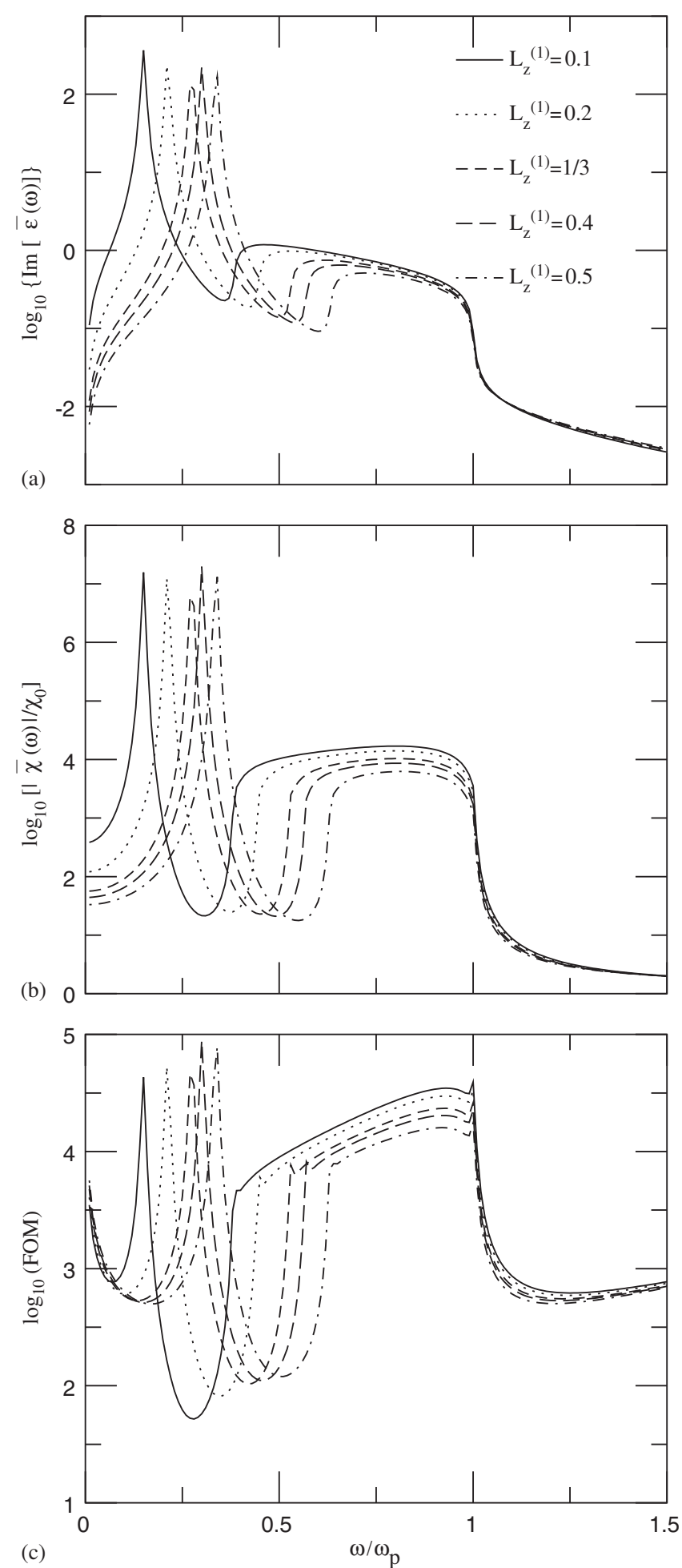

Fig. 23. Results for the MGA2 [Eq. (84)] [99]: (a) Linear optical absorption $\operatorname{Im}[\bar{\epsilon}(\omega)]$; (b) enhancement of the third-order optical nonlinearity $|\bar{\chi}(\omega)| / \chi_{0}$; and (c) FOM $\equiv|\bar{\chi}(\omega)| /\left\{\chi_{0} \operatorname{Im}[\bar{\epsilon}(\omega)]\right\}$ versus the normalized incident angular frequency $\omega / \omega_{p}$ for layer dielectric profile $p(z)=a z^{m}$, for different $L_{z}^{(1)}$. Parameters: $a=0.8, m=1, \gamma / \omega_{p}=0.01$, and $\epsilon_{2}=(3 / 2)^{2}$. 

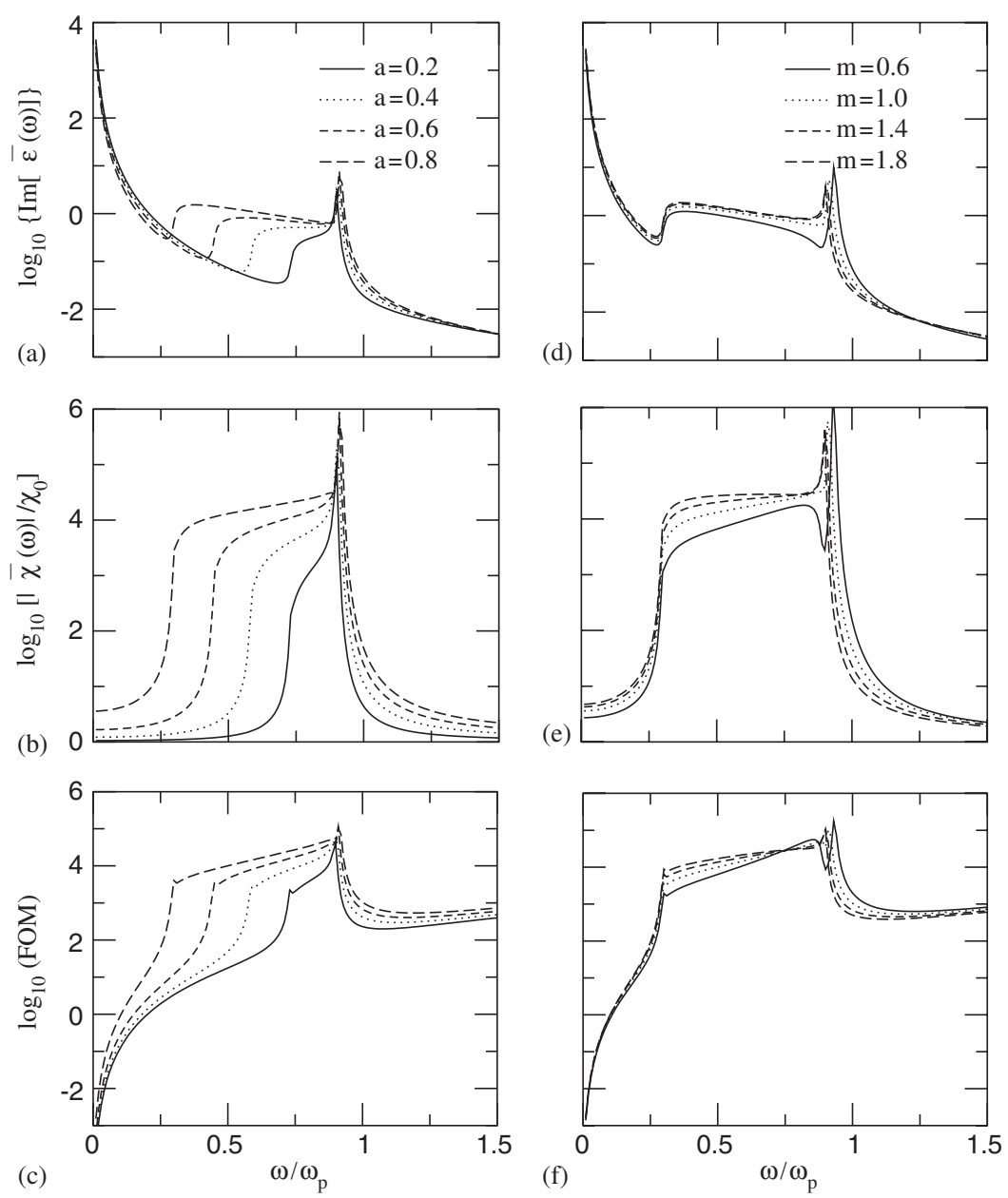

Fig. 24. Results for the MGA1 [Eq. (83)] [99]: (a) and (d) $\operatorname{Im}[\bar{\epsilon}(\omega)]$; (b) and (e) $|\bar{\chi}(\omega)| / \chi_{0}$, and (c) and (f) FOM $\equiv|\bar{\chi}(\omega)| /\left\{\chi_{0} \operatorname{Im}[\bar{\epsilon}(\omega)]\right\}$ versus $\omega / \omega_{p}$ (a)-(c) for different $a$ at $m=1.0$, and (d)-(f) for different $m$ at $a=0.8$. Parameters: $L_{z}=0.1, \gamma / \omega_{p}=0.01$, and $\epsilon_{2}=(3 / 2)^{2}$.

nonzero $n$ (i.e., $n=4,8,12$ ) is not very distinct (Fig. 27). Interestingly, the $L_{z}$ gradation gives rise to an additional peak which appears at a lower frequency. For the MGA2 (Fig. 28), the surface plasmon resonant bands in optical absorption and nonlinearity are clearly visible for various $L_{z}(z)$ profiles, see Fig. 28(a-b). In the presence of gradation, i.e., $n$ becomes nonzero, the prominent plasmon absorption peak at $n=0$ has been broadened into a plasmon band, and an additional peak is induced to appear at lower frequency. Concomitantly, a plasmon band and a peak in optical nonlinearity are also caused to appear [Fig. 28(b)] and hence the FOM can be enhanced accordingly, see Fig. 28(c). On the other hand, we also find that the plasmon bands in optical absorption and nonlinearity can be further broadened (and enhanced) by adopting a wider gradation profile such as $L_{z}(z)=0.5 z^{n}$ (no figures shown here). For this sort of profile, there are prolate particles at the top, but oblate particles at the bottom of the film. It is possible to realize such oblate particles near the bottom of the film due to the reaction stress from the substance.

Finally, in Figs. 23(a), 25(a) and 28(a), there are always a plasmon band plus an absorption peak as long as the gradation profile exists. Recently, an absorption peak plus a slim plasmon absorption band was indeed observed [88], when one investigated the optical extinction spectra for ensembles of core-shell colloids with Au cores and shells embedded in an index-matching fluid. But, after irradiation with $30 \mathrm{MeV}$ (mega electron volt) $\mathrm{Cu}$ ions, a broadening of the plasmon absorption band was also observed, which was thought to attribute to the formation of Au nanorods. To account for this behavior, we believe the particle shape, and gradation in the depolarization factor of metals and in the volume fraction of the metallic (or dielectric) component should be expected to play an important role. 

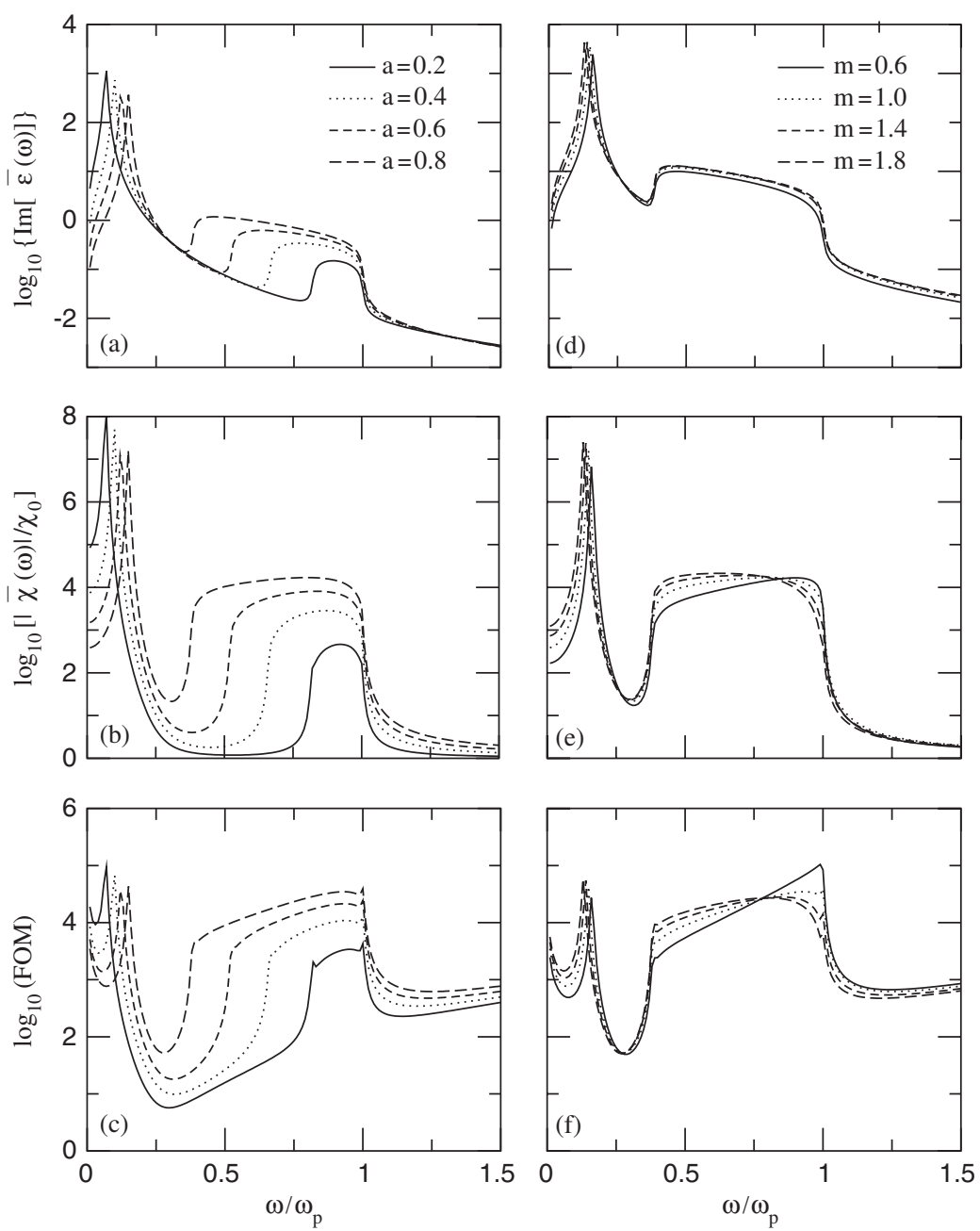

Fig. 25. Results for the MGA2 [Eq. (84)] [99]: (a) and (d) $\operatorname{Im}[\bar{\epsilon}(\omega)]$; (b) and (e) $|\bar{\chi}(\omega)| / \chi_{0}$; and (c) and (f) $\operatorname{FOM} \equiv|\bar{\chi}(\omega)| /\left\{\chi_{0} \operatorname{Im}[\bar{\epsilon}(\omega)]\right\}$ versus $\omega / \omega_{p}$ (a)-(c) for different $a$ at $m=1.0$, and (d)-(f) for different $m$ at $a=0.8$. Parameters: $L_{z}=0.1, \gamma / \omega_{p}=0.01$, and $\epsilon_{2}=(3 / 2)^{2}$.

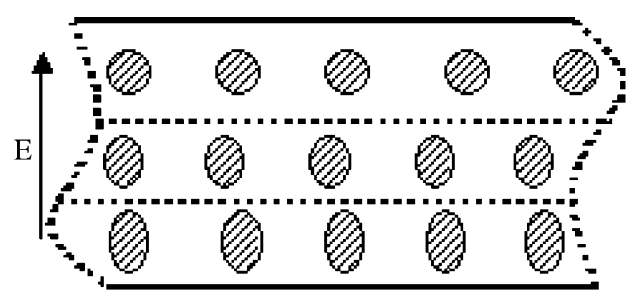

Fig. 26. Schematic graph to show the geometry of a metal-dielectric composite film with a variation of depolarization factor of particles along $z$ axis perpendicular to the film [99]. The electric field $E$ is parallel to the gradient ( $z$ axis), thus being perpendicular to the film.

In a word, the sharp plasmon peak comes naturally from the existence of metal-dielectric interfaces. In the case of graded metallic films, there should be a broad band only, but no sharp peak. So, for the graded metal-dielectric composite film under present consideration, both the plasmon peak and the broad plasmon band should appear as predicted above. 

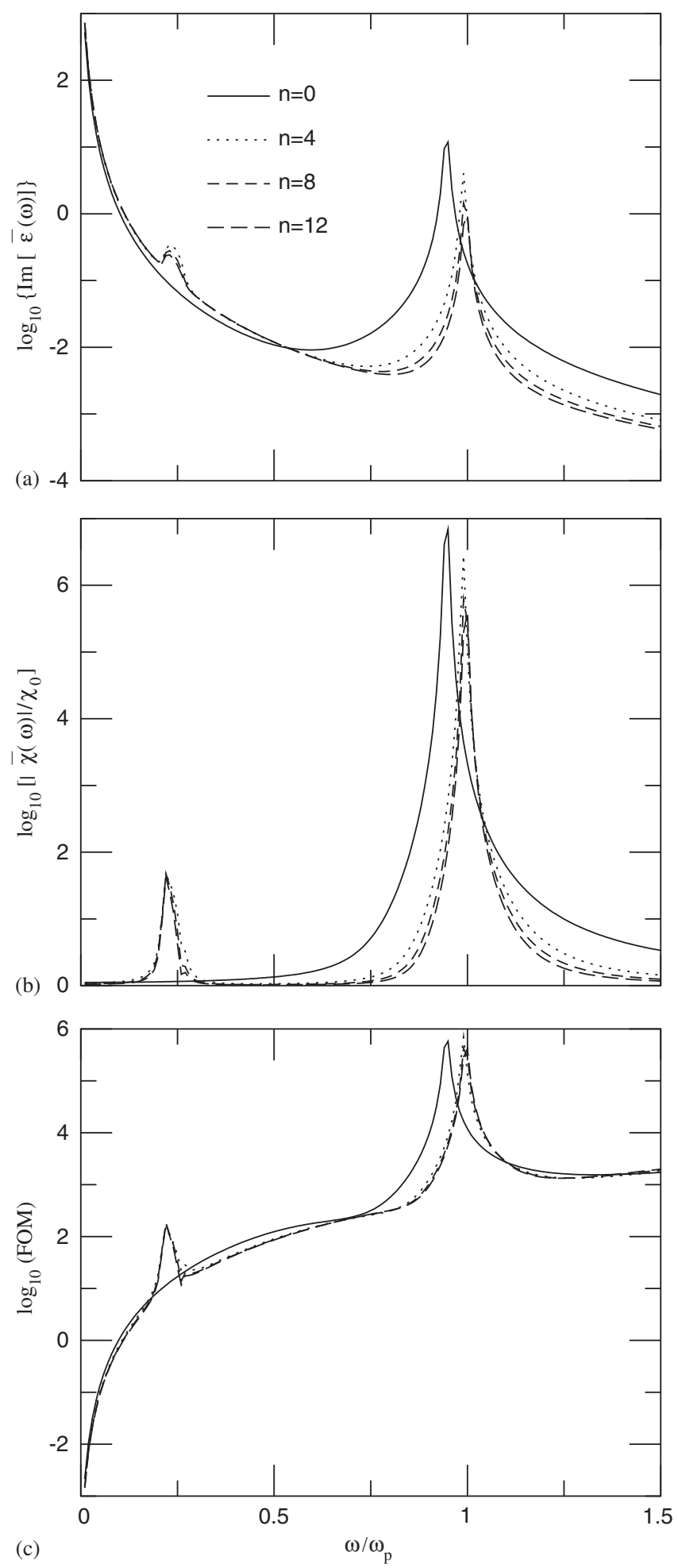

Fig. 27. Results for the MGA1 [Eq. (83)] [99]: (a) Linear optical absorption $\operatorname{Im}[\bar{\epsilon}(\omega)]$; (b) enhancement of the third-order optical nonlinearity $|\bar{\chi}(\omega)| / \chi_{0}$; and (c) $\mathrm{FOM} \equiv|\bar{\chi}(\omega)| /\left\{\chi_{0} \operatorname{Im}[\bar{\epsilon}(\omega)]\right\}$ versus the normalized incident angular frequency $\omega / \omega_{p}$ for the gradation profile of the depolarization factor of dielectric particles $L_{z}^{(2)}(z)=(1 / 3) z^{n}$, for different $n$. Parameters: $p(z)=0.85, \gamma / \omega_{p}=0.01$, and $\epsilon_{2}=(3 / 2)^{2}$. 

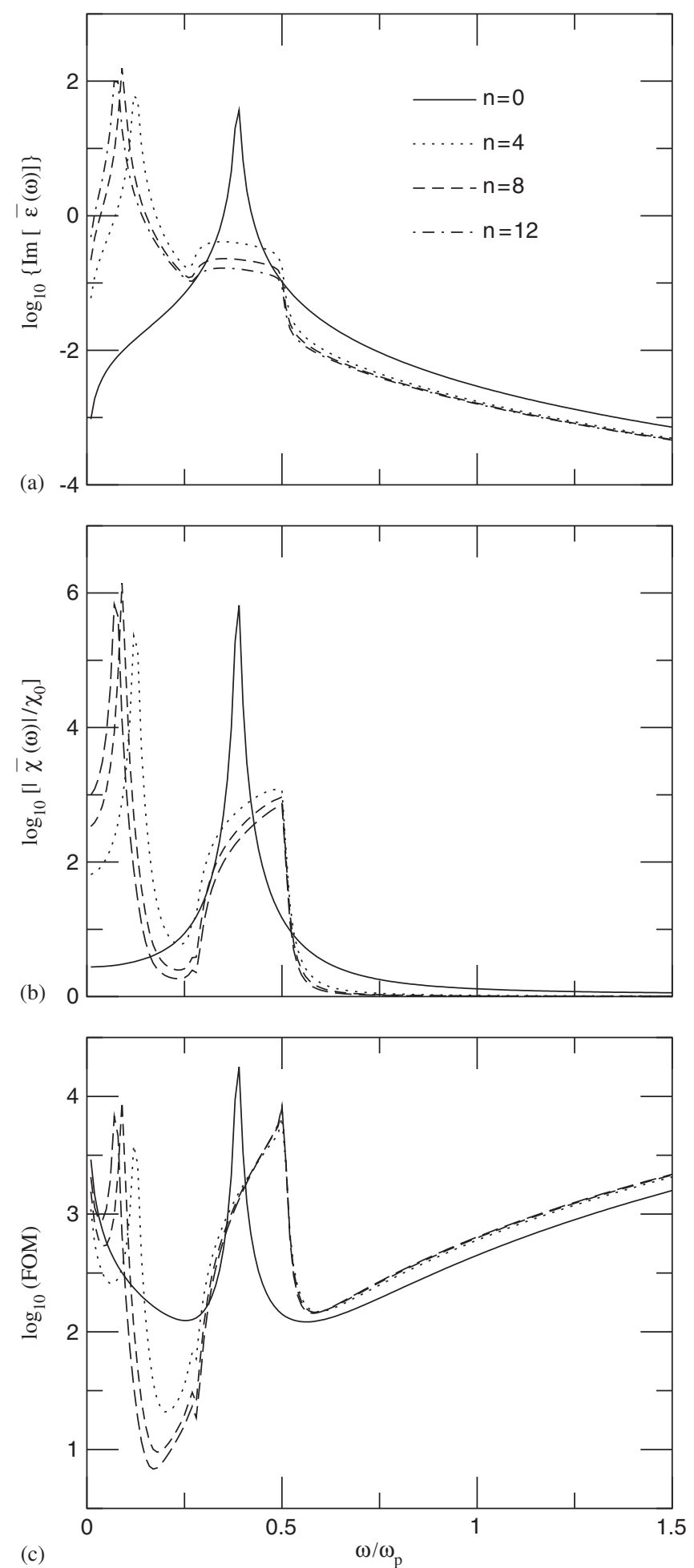

Fig. 28. Results for the MGA2 [Eq. (84)] [99]: (a) Linear optical absorption $\operatorname{Im}[\bar{\epsilon}(\omega)$ ]; (b) enhancement of the third-order optical nonlinearity $|\bar{\chi}(\omega)| / \chi_{0}$; and (c) FOM $\equiv|\bar{\chi}(\omega)| /\left\{\chi_{0} \operatorname{Im}[\bar{\epsilon}(\omega)]\right\}$ versus the normalized incident angular frequency $\omega / \omega_{p}$ for the gradation profile of the depolarization factor of metallic particles $L_{z}^{(1)}(z)=(1 / 3) z^{n}$, for different $n$. Parameters: $p(z)=0.85, \gamma / \omega_{p}=0.01$, and $\epsilon_{2}=(3 / 2)^{2}$. 
We have studied the effective nonlinear optical response of a graded metal-dielectric composite film of anisotropic particles. Based on the MGA1 and MGA2 [Eqs. (83) and (84)], we derived the local electric field inside the film, and hence obtained the effective linear dielectric constant [Eq. (96)] and third-order nonlinear susceptibility [Eq. (98)] of the graded composite film.

In comparison with textbook formulae, the formulae [Eqs. (83) and (84)] only differ from the $z$-dependent volume fraction $p(z)$, in the sense that we could discuss the gradation which is perpendicular to the film and then leads to nonlinearity enhancement. As a matter of fact, the present results do not depend crucially on the particular form of the dielectric gradation profile $p(z)$ or the depolarization-factor gradation profile $L_{z}(z)$. The only requirement is that we must have a compositional or shape-dependent gradation to yield a broad plasmon band for the composite film. It should be remarked that the optical response of the graded structure depends on polarization of the incident light, because the incident optical field can always be resolved into two polarizations. However, a large nonlinearity enhancement occurs only when the electric field is parallel to the direction of the gradient [4], and the other polarization does not produce nonlinearity enhancement at all [4]. The nonlinear susceptibilities of both the parallel and perpendicular polarizations are related to the nonlinear phase shift which can be measured by using a $z$-scan method [4].

Following Roorda et al. [88], one could fabricate the film under the present discussion by using mega-electron-volt ion irradiation. Its third-order nonlinear susceptibility could also be measured by a degenerated four-wave-mixing method, which has been used for the $\mathrm{Au} / \mathrm{SiO}_{2}$ composite film [12]. It is of interest to extend the present theory to composites in which graded spherical particles are embedded in a host medium [20] to account for mutual interactions among graded particles.

To sum up, we have studied the effective linear dielectric constant and third-order nonlinear susceptibility of a graded metal-dielectric composite film of anisotropic particles with weak nonlinearity by invoking the local field effects exactly within the Maxwell-Garnett theory. We have numerically demonstrated that this kind of film can serve as a novel optical material for producing a broad structure in both the linear and nonlinear response and an enhancement in the nonlinear response.

\subsection{Compositionally graded metal-dielectric films: effects of microstructure}

One of the crucial elements for control of the linear and/or nonlinear optical responses is the microstructure of composites (e.g., see Refs. [3,57] and references therein). For discussing the effect of microstructure, the usual two methods are the well-known Maxwell-Garnett approximation (MGA) [27,28] and Bruggeman effective medium approximation (EMA) [29]. It is worth noting that the MGA is an asymmetrical theory (Section 2.1) whereas the EMA is a symmetrical theory (Section 2.2).

As mentioned above, the nonlinearity enhancement often occurs concomitantly with a strong absorption, and unfortunately this behavior renders the corresponding FOM to be too small to be useful. To circumvent this problem, we shall consider a kind of compositionally graded metal-dielectric film in which a dielectric component is introduced as particulates embedded in the metallic component. To our interest, most recently compositionally graded ferroelectric films were investigated, and new phenomenon was shown indeed [104]. In this regard, we expect the compositionally graded metal-dielectric film under our investigation can show some new interesting phenomenon concerning the nonlinear and/or linear optical responses, too.

Let us consider a nonlinear graded film with width $L$, in which the dielectric particles of dielectric constant $\epsilon_{2}$ is embedded in the host metal of $\epsilon_{1}(\omega)$, and the volume fraction of the embedded particles $p_{2}(z)$ varies along $z$-axis. Here the gradient of gradation is in the direction perpendicular to the film, i.e., in $z$-axis. In this connection, the local constitutive relation between the displacement $\mathbf{D}$ and electric field $\mathbf{E}$ is given by

$$
\mathbf{D}(z, \omega)=\epsilon(z, \omega) \mathbf{E}(z, \omega)+\chi(z, \omega)|\mathbf{E}(z, \omega)|^{2} \mathbf{E}(z, \omega),
$$

where $\epsilon(z, \omega)$ and $\chi(z, \omega)$ are, respectively, the linear dielectric constant and third-order nonlinear susceptibility of a layer inside the graded film. It is worth mentioning that both $\epsilon(z, \omega)$ and $\chi(z, \omega)$ are gradation profiles as a function of position $z$.

Let us further assume that the weak nonlinearity condition is satisfied. That is, the contribution of the second term (nonlinear part $\left.\chi(z, \omega)|\mathbf{E}(z, \omega)|^{2}\right)$ in the right-hand side of Eq. (100) is much less than that of the first term (linear part $\epsilon(z, \omega))[74]$. Next, we restrict our discussion to the quasi-static approximation, i.e., $d / \lambda \leqslant 1$, where $d$ is the characteristic size of the particle and $\lambda$ is the wavelength of the incident light. In the quasi-static approximation, the whole graded 
structure can be regarded as an effective homogeneous one with effective (overall) linear dielectric constant $\bar{\epsilon}(\omega)$ and effective (overall) third-order nonlinear susceptibility $\bar{\chi}(\omega)$. That is, $\bar{\epsilon}(\omega)$ and $\bar{\chi}(\omega)$ is defined as [74]

$$
\langle\mathbf{D}\rangle=\bar{\epsilon}(\omega) \mathbf{E}_{0}+\bar{\chi}(\omega)\left|\mathbf{E}_{0}\right|^{2} \mathbf{E}_{0},
$$

where $\langle\cdots\rangle$ stands for the spatial average of $\cdots$, and $\mathbf{E}_{0}=E_{0} \hat{e}_{z}$ the applied field along $z$-axis.

Now let us take into account the detailed microstructures in order to obtain $\bar{\epsilon}(\omega)$ and $\bar{\chi}(\omega)$. First, we consider an asymmetrical microstructure [27,28] in which one component constitutes inclusion particles while the other serves as a host. For such a microstructure, the MGA (Maxwell-Garnett approximation) is valid. In detail, for each layer of the graded metal-dielectric film, the effective dielectric constant $\epsilon(z, \omega)$ is the solution of the MGA equation,

$$
\frac{\epsilon(z, \omega)-\epsilon_{1}(\omega)}{\epsilon(z, \omega)+2 \epsilon_{1}(\omega)}=p_{2}(z) \frac{\epsilon_{2}-\epsilon_{1}(\omega)}{\epsilon_{2}+2 \epsilon_{1}(\omega)},
$$

On the other hand, we focus on a symmetrical microstructure in which the two sorts of particles can be exchanged, yielding, however, no effect on the effective dielectric constant $\epsilon(z, \omega)$ of each layer. For treating such a symmetric microstructure, the EMA (effective medium approximation or Bruggeman approximation) [29] works for calculating $\epsilon(z, \omega)$ by solving the self-consistent equation such that

$$
\left(1-p_{2}(z)\right) \frac{\epsilon_{1}(\omega)-\epsilon(z, \omega)}{\epsilon_{1}(\omega)+2 \epsilon(z, \omega)}+p_{2}(z) \frac{\epsilon_{2}-\epsilon(z, \omega)}{\epsilon_{2}+2 \epsilon(z, \omega)}=0 .
$$

Regarding Eqs. (102) and (103), we should remark more. In fact, it is not possible to calculate $\epsilon(z, \omega)$ exactly in terms of the layer dielectric profile $p_{2}(z)$. Nevertheless, to obtain an estimate of $\epsilon(z, \omega)$, we can take a small volume element inside the layer, at a position $z$. Further, this small volume element can be seen as a composite where the dielectric particles are randomly embedded in the metallic component. Accordingly, the volume fraction of the dielectric particles is $p_{2}(z)$. In this regard, the above-mentioned MGA and EMA should be expected to hold well for computing $\epsilon(z, \omega)$.

Owing to the simple graded structure, we can use the equivalent capacitance of series combination to calculate the linear response (i.e., optical absorption),

$$
\frac{1}{\bar{\epsilon}(\omega)}=\frac{1}{L} \int_{0}^{L} \frac{\mathrm{d} z}{\epsilon(z, \omega)} .
$$

To investigate the nonlinear optical response, we first calculate local electric field $E(z, \omega)$ by means of the identity

$$
\epsilon(z, \omega) E(z, \omega)=\bar{\epsilon}(\omega) E_{0}
$$

due to the virtue of the continuity of the electric displacement. In view of the existence of nonlinearity inside the graded film, the effective nonlinear response $\bar{\chi}(\omega)$ can be given by [74]

$$
\bar{\chi}(\omega) \mathbf{E}_{0}^{4}=\left\langle\chi(z, \omega)\left|\mathbf{E}_{\operatorname{lin}}(z)\right|^{2} \mathbf{E}_{\operatorname{lin}}(z)^{2}\right\rangle,
$$

where $\mathbf{E}_{\text {lin }}$ denotes the linear local electric field. Next, we take one step forward to express the effective nonlinear response as an integral over the film,

$$
\bar{\chi}(\omega)=\frac{1}{L} \int_{0}^{L} \mathrm{~d} z \chi(z, \omega)\left|\frac{\bar{\epsilon}(\omega)}{\epsilon(z, \omega)}\right|^{2}\left(\frac{\bar{\epsilon}(\omega)}{\epsilon(z, \omega)}\right)^{2} .
$$

For the following numerical calculations, we adopt a Drude-type dielectric function for metallic particles, namely,

$$
\epsilon_{1}(\omega)=1-\frac{\omega_{p}^{2}}{\omega(\omega+\mathrm{i} \gamma)},
$$

where $\omega_{p}$ denotes the bulk plasmon frequency, and $\gamma$ the damping constant. In addition, we set $\gamma=0.01 \omega_{p}$ (typical value for noble bulk metals) and $\epsilon_{2}=(3 / 2)^{2}$ (dielectric constant of glass).

Now we are in a position to do some numerical calculations in an attempt to discuss the effect of the above-mentioned microstructures. Set $\chi(z, \omega)$ to be a constant $\chi_{1}$, so that we could emphasize the enhancement of the optical nonlinearity. 
Regarding the layer dielectric profile, take a power form $p_{2}(z)=a z^{m}$. Without loss of generality, the layer width $L$ is set to be unity.

In Figs. 29-32, we plot (a) the optical absorption $\sim \operatorname{Im}[\bar{\epsilon}(\omega)]$, (b) the modulus of the effective third-order optical nonlinearity enhancement $|\bar{\chi}(\omega)| / \chi_{1}$, and (c) the FOM (figure of merit) $|\bar{\chi}(\omega)| /\left\{\chi_{1} \operatorname{Im}[\bar{\epsilon}(\omega)]\right\}$ as a function of the incident angular frequency $\omega$, respectively. Here $\operatorname{Im}[\cdots]$ means the imaginary part of $\cdots$.

It is well known that the MGA correctly predicts the surface plasmon resonance of bulk metal-dielectric composite, and this properties is shown as well in our model for a metal-dielectric film. Figs. 29 and 30 show the optical properties based on the MGA. In Fig. 29, we display the effect of the coefficient $a$. When the layer dielectric profile $p_{2}(z)$ is taken into account, a broad resonant plasmon band is observed always. In other words, the broad band is caused to appear by the effect of the positional dependence of the dielectric or metal. Also, we find that increasing $a$ causes the resonant band not only to be enhanced, but also red-shifted (namely, located at a lower frequency region). In a word, although the enhancement of the effective third-order optical nonlinearity is often accompanied with the appearance of the optical absorption, the FOM is still possible to be very attractive due to the presence of the positional dependence of the dielectric or metallic components. Moreover, it is worth noting that a prominent surface plasmon resonant peak appears at somewhat higher frequencies in addition to the surface plasmon band. As $a$ increases, this peak is blue-shifted (i.e., locates at a higher frequency region) accordingly.

Similarly, Fig. 30 displays the influence of $m$. It is apparent to see that the broad resonant plasmon band can be enhanced significantly by adjusting $m$. However, no distinct red-shift occurs for the plasmon band as $m$ varies. In contrast, we notice that increasing $m$ can make the surface plasmon resonant peak red-shifted.

For the symmetrical microstructure (EMA model), we also display the effects of $a$ (Fig. 31) and $m$ (Fig. 32), respectively. For this kind of microstructure, a plasmon band exists as well. However, the surface plasmon resonance becomes broad and weak, and the resonance peak disappears at a large volume fraction of metallic particles (e.g., $a=0.2, m=1.0$ ). This is different from the MGA prediction that whatever the volume fraction of the metal component is there always exists a sharp resonance. That is, such a difference is caused by introducing the two different (asymmetrical and symmetrical) microstructures. Moreover, it is well known that there is a percolation threshold predicted by the EMA, at which the properties of the metal-dielectric composite change significantly. For metal-dielectric composites the percolation threshold is $p_{2}(z)=2 / 3$ [i.e., $1-p_{2}(z)=1 / 3$ ] at which the conductivity of the composite becomes nonzero. In other words, as $1-p_{2}(z)>1 / 3$ the composite behaves as a metal rather that a dielectric. Thus, in Figs. 31 and 32, there is no apparent resonance peak especially when the volume fraction of the metal is large. Also, the nonlinearity enhancement is unimpressive and the FOM is generally small. Nevertheless, the plasmon band always exists, too.

For the Maxwell-Garnett model, the microstructure of interest should be asymmetrical. That is, the dielectric particles are surrounded by the metallic component. In other words, the dielectric particles are randomly dispersed in a metallic host so that the dielectric particles cannot touch each other. On the other hand, for the Bruggeman model, the microstructure should be symmetrical. That is, both the dielectric particles and metallic particles are mixed randomly. It is not possible to distinguish the embedded component with the host medium. It is known that the Maxwell-Garnett model (without gradation) can predict a sharp plasmon resonance peak whereas the Bruggeman model will give a broad plasmon band. For the Maxwell-Garnett model with gradation, a broad resonant plasmon band is observed, see Figs. 29-30. It is because the inhomogeneity due to gradation leads to a further broadening of the plasmon resonance peak. In more detail, as $a$ or $m$ increases, see Fig. 29 or 30, the resonant frequency takes on values within a broader range across the film, and hence leads to a broad plasmon band. The further broadening in the plasmon band also occurs in the Bruggeman model.

We have discussed the effective linear and nonlinear optical responses of a compositionally graded metal-dielectric film in an attempt to study the effect of microstructure. For two asymmetrical and symmetrical microstructures, we used the MGA and EMA to calculate the effective responses, respectively.

The appearance of plasmon bands is interesting, and comes about from the gradual changes in the volume fraction of the metallic component in one direction, i.e. like a one-dimensional tight binding band of surface plasmon modes at each layer. As a matter of fact, the present results do not depend crucially on the particular form of the layer dielectric profile $p(z)$. However, the microstructure can significantly affect the linear and nonlinear optical response, as showed above. As the volume fraction of the metal component increases, the MGA (or EMA) predicts a sharp (or broad and weak) resonance. However, a plasmon band was observed always, regardless of the detailed microstructure. To obtain such results, the only requirement is that one must have a composition-dependent layer inside the graded film. 

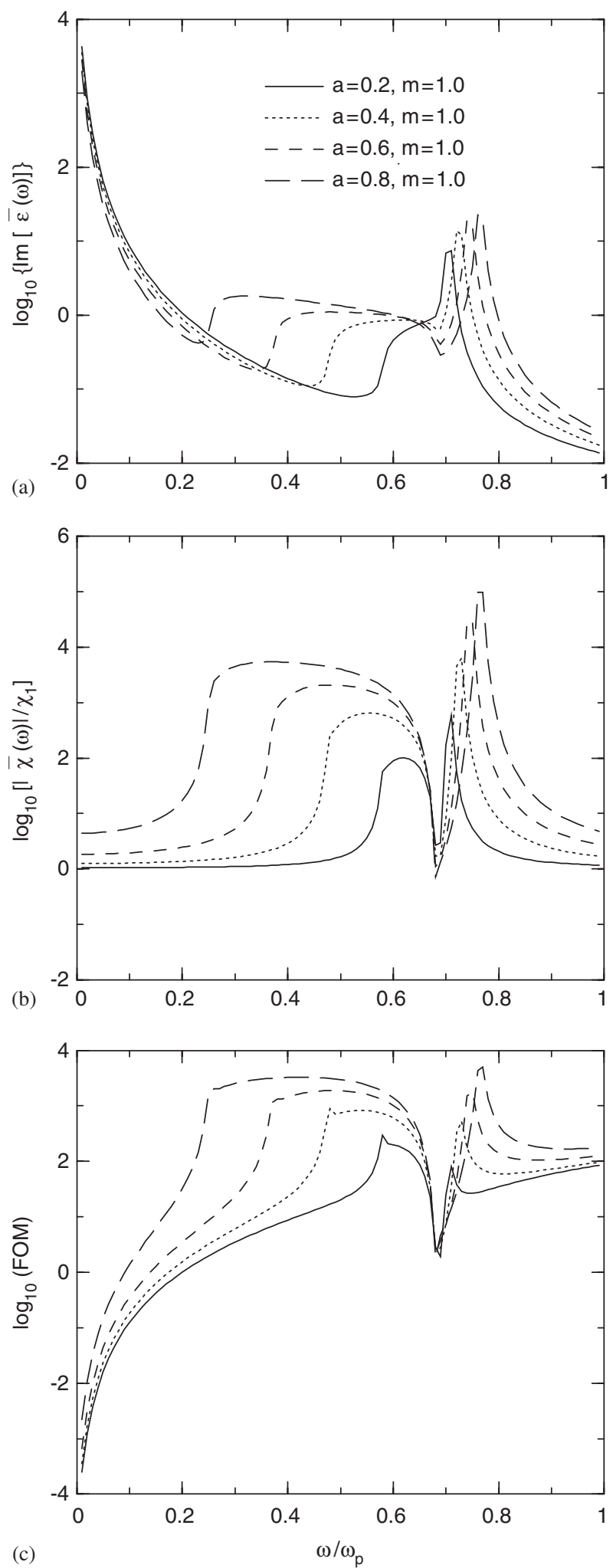

Fig. 29. Results of the MGA: (a) Linear optical absorption $\operatorname{Im}[\bar{\epsilon}(\omega)]$; (b) enhancement of the third-order optical nonlinearity $|\bar{\chi}(\omega)| / \chi_{1}$; and (c) FOM (figure of merit) $\equiv|\bar{\chi}(\omega)| /\left\{\chi_{1} \operatorname{Im}[\bar{\epsilon}(\omega)]\right\}$ versus the normalized incident angular frequency $\omega / \omega_{p}$ for layer dielectric profile $p_{2}(z)=a z^{m}$. Parameters: $\gamma / \omega_{p}=0.01$ and $\epsilon_{2}=(3 / 2)^{2}$. 

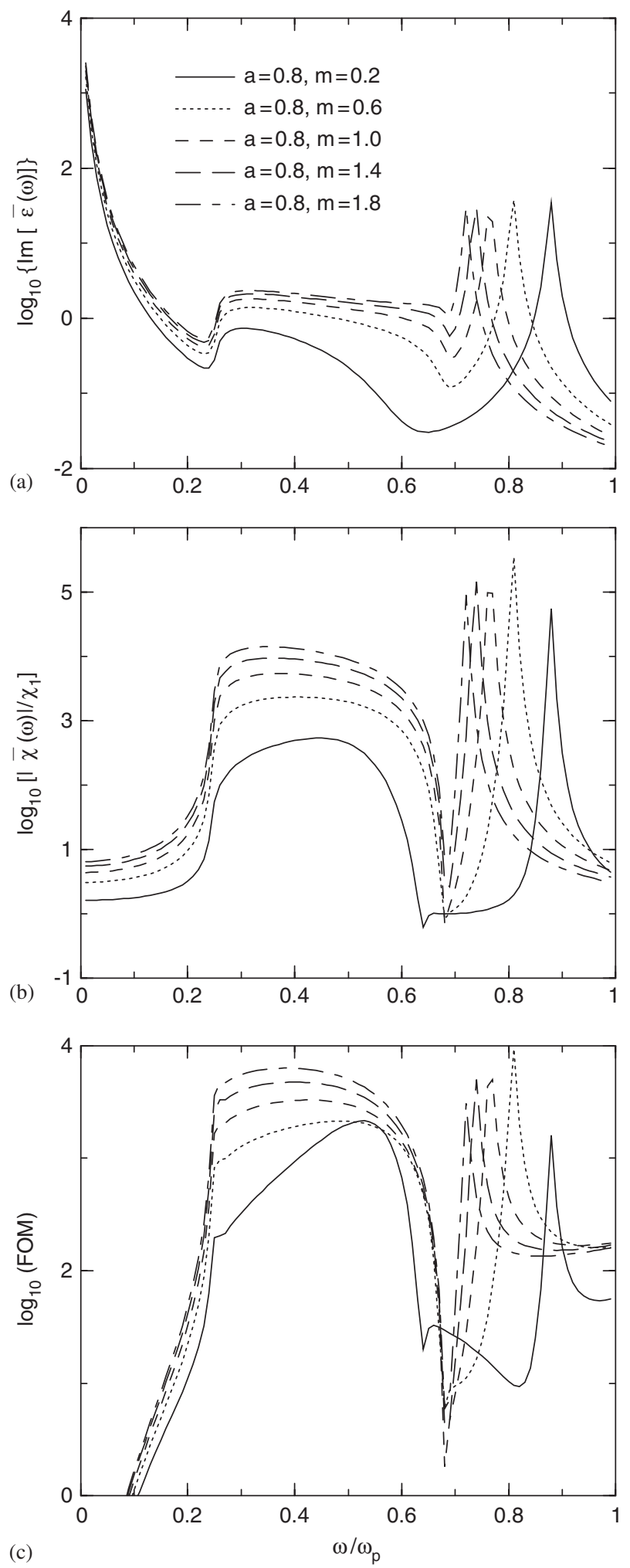

Fig. 30. Results of the MGA. Same as Fig. 29, but for different $m$ at $a=0.8$. 

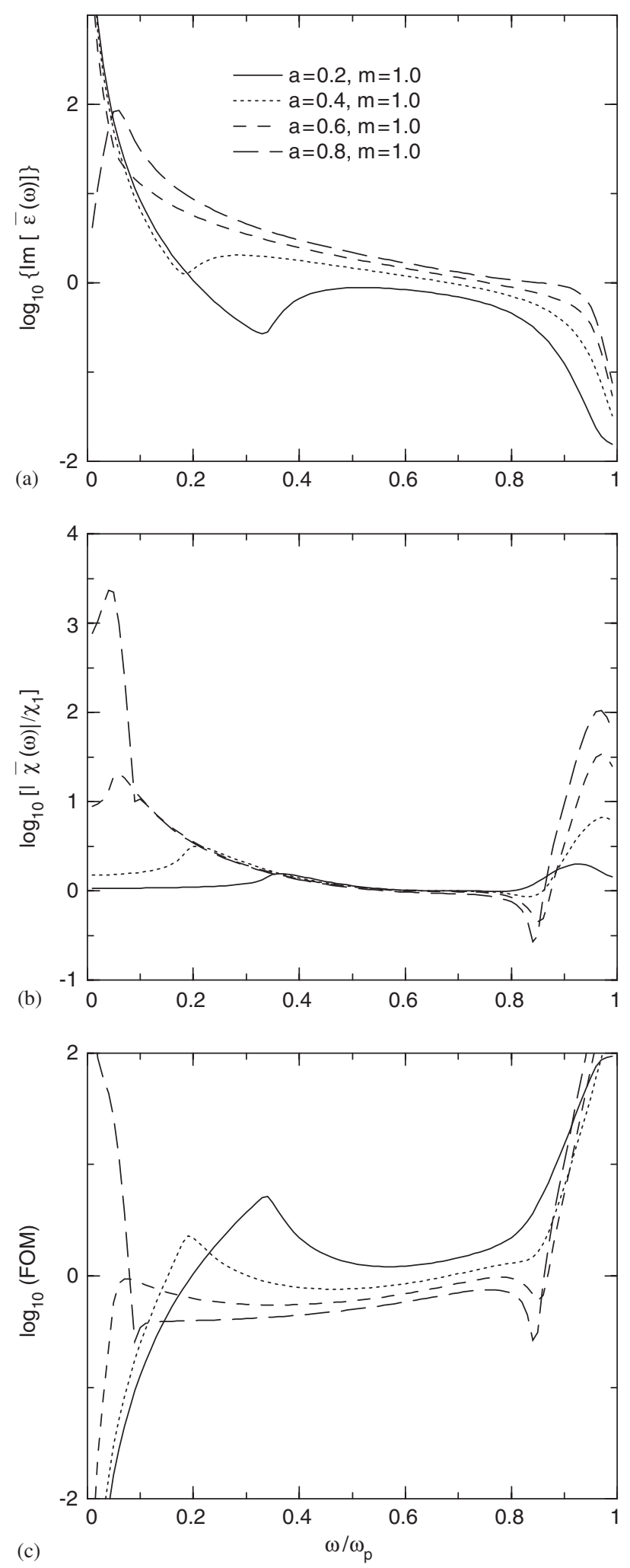

Fig. 31. Results of the EMA. Others are the same as Fig. 29. 

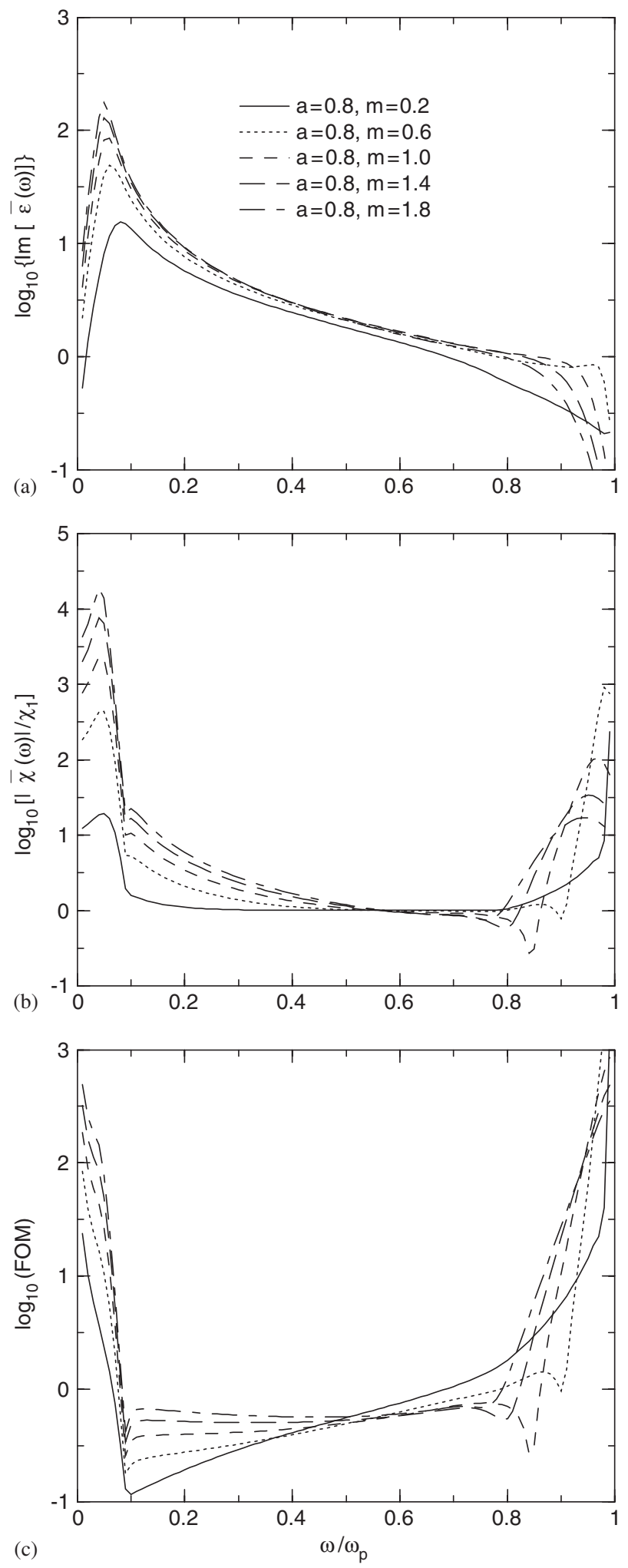

Fig. 32. Results of the EMA. Others are the same as Fig. 30. 
In fact, a direct numerical evaluation of the linear problem giving $E_{\text {lin }}$ can give an independent check for the validity of Eqs. (104) and (105). In so doing, one needs to invoke a random resistor network for the genuine random composite.

It is also interesting to extend the present consideration to composites in which graded spherical particles are embedded in a host medium [20].

To calculate $\epsilon(z, \omega)$ for an asymmetrical microstructure, we used the MGA [Eq. (102)] for which the metal (or dielectric) component serves as a host (or inclusion). Inversely, we could see the metal (or dielectric) component as an inclusion (or host), and use the same form as Eq. (102) by exchanging $\epsilon_{1}(\omega)$ and $\epsilon_{2}$, and $p_{2}(z)$ and $1-p_{2}(z)$. In so doing, as $\epsilon_{1}(\omega)>\epsilon_{2}$ the former MGA [Eq. (102)] always gives an upper bound while the latter offers a lower bound, and vice versa. But the exact result must lie between the two bounds, as already pointed out by Hashin and Shtrikman [102].

To sum up, we have studied a compositionally graded metal-dielectric film by investigating two asymmetrical and symmetrical microstructures, respectively, and found that the effective linear and nonlinear optical responses are sensitive to the microstructure. Thus, it is possible to gain a large nonlinearity enhancement and optimal FOM by choosing an appropriate microstructure in compositionally graded metal-dielectric films.

\subsection{Composite media of graded spherical particles}

Graded materials [39,73], whose properties vary gradually as a function of position, have received much attention as one of the advanced heterogeneous composites in various engineering applications by using the gradients in thermal [105], electric [106] and mechanical properties [107]. This gradation may occur naturally or may be a product of manufacturing processes. It was reported $[4,5,21,23]$ that graded (inhomogeneous) materials can show stronger nonlinear optical responses than the corresponding homogeneous ones. Also, it is desirable and of interest to use dielectric-coated metallic nanoparticles with varying shell thickness to form a dielectric constant gradient [56]. In nature, there are also many graded materials, such as liquid crystal droplets [108] and biological cells [24], because of the inhomogeneous compartment inside them.

Physically, graded materials are quite different from the homogeneous ones and other conventional composites. Therefore, the composite media consisting of graded particles can be more useful and interesting than those of homogeneous inclusions. The traditional theories used to deal with the homogeneous materials [109], however fail to deal with composites of graded inclusions directly. To treat these composites, we have recently developed a first-principles approach [19,40,110] and a differential effective dipole theory (Section 2.4) [40,111].

The problem becomes more complicated by the presence of nonlinearity in realistic composites. Besides inhomogeneity, such nonlinearity plays also an important role in the effective material properties of composite media $[3,6,17,68,74,75]$. It is thus necessary to establish a new theory to study the effective nonlinear properties of graded composite media. In fact, the introduction of dielectric gradation profiles in nonlinear composites is able to provide an alternative way to control the local field fluctuation, and hence let us obtain the desired effective nonlinear response.

In fact, the previous one-shell model [78] and multi-shell model [76], which were used to study the effective nonlinear optical property, can be seen as an initial model of graded inclusions. In Section 5.3, we shall put forth a nonlinear differential effective dipole approximation (NDEDA) to investigate the effective linear and nonlinear dielectric properties of composite media containing a very small volume fraction of nonlinear graded spherical particles (inclusions). For such particles, the linear and nonlinear physical properties will continuously vary along their radius.

\subsubsection{Model and definition of effective linear and nonlinear responses}

Let us consider a nonlinear composite system, in which identical graded spherical inclusions with radius $a$, are randomly embedded in a linear host medium of dielectric constant $\epsilon_{2}$. The local constitutive relation between the displacement (D) and the electric field (E) inside the graded particle is given by

$$
\mathbf{D}=\epsilon(r) \mathbf{E}+\chi(r)|\mathbf{E}|^{2} \mathbf{E}
$$

where $\epsilon(r)$ and $\chi(r)$ are, respectively, the linear dielectric constant and third-order nonlinear susceptibility. Note both $\epsilon(r)$ and $\chi(r)$ are radial functions. Here we assume that the weak nonlinearity condition is satisfied [74]. In other words, the contribution of the second (nonlinear) part $\left[\chi_{s}(r)|\mathbf{E}|^{2}\right]$ in the right-hand side of Eq. (109) is much less than that of the first (linear) part $\epsilon(r)$. We restrict further our discussion to the quasi-static approximation, under which the whole 
composite medium can be regarded as an effective homogeneous one with effective linear dielectric constant $\epsilon_{e}$ and effective third-order nonlinear susceptibility $\chi_{e}$. To show the definitions of $\epsilon_{e}$ and $\chi_{e}$, we have [74]

$$
\langle\mathbf{D}\rangle=\epsilon_{e} \mathbf{E}_{0}+\chi_{e}\left|\mathbf{E}_{0}\right|^{2} \mathbf{E}_{0},
$$

where $\langle\cdots\rangle$ represents the spatial average, and $\mathbf{E}_{0}=E_{0} \mathbf{e}_{z}$ is the external applied field along $z$ axis.

The effective linear dielectric constant $\epsilon_{e}$ is given by

$$
\epsilon_{e} \mathbf{E}_{0}=\frac{1}{V} \int_{V} \epsilon_{i} \mathbf{E}_{\mathrm{lin}, \mathrm{i}} \mathrm{d} V=f\left\langle\epsilon(r) \mathbf{E}_{\mathrm{lin}, 1}\right\rangle+(1-f) \epsilon_{2}\left\langle\mathbf{E}_{\mathrm{lin}, 2}\right\rangle,
$$

where $f$ is the volume fraction of the graded particles and the subscript stands for the linear local field [i.e., obtained for the same system but with $\chi(r)=0]$.

In view of the existence of nonlinearity inside the graded particles, $\chi_{e}$ can then be written as $[74,100]$

$$
\chi_{e} \mathbf{E}_{0}^{4}=\frac{1}{V} \int_{V} \chi_{i}|\mathbf{E}|_{\operatorname{lin}, i}^{2} \mathbf{E}_{\operatorname{lin}, i}^{2} \mathrm{~d} V=\frac{1}{V} \int_{\Omega_{i}} \chi(r)|\mathbf{E}|_{\operatorname{lin}, 1}^{2} \mathbf{E}_{\operatorname{lin}, 1}^{2} \mathrm{~d} V=f\left\langle\chi(r)|\mathbf{E}|_{\operatorname{lin}, 1}^{2} \mathbf{E}_{\operatorname{lin}, 1}^{2}\right\rangle .
$$

In the next section, we will develop a NDEDA (nonlinear differential effective dipole approximation), in an attempt to derive the equivalent linear dielectric constant $\bar{\epsilon}(a)$ and third-order nonlinear susceptibility $\bar{\chi}(a)$ of the nonlinear graded inclusions. Then, the effective linear dielectric constant and third-order nonlinear susceptibility of the composite media of nonlinear graded inclusions will be derived accordingly in the dilute limit.

\subsubsection{Nonlinear differential effective dipole approximation}

To establish the NDEDA, we first mimic the gradation profile by a multi-shell construction. That is, we build up the dielectric profile by adding shells gradually [112]. We start with an infinitesimal spherical core with linear dielectric constant $\epsilon(0)$ and third-order nonlinear susceptibility $\chi(0)$, and keep on adding spherical shells with linear dielectric constant $\epsilon(r)$ and third-order nonlinear susceptibility $\chi(r)$ at radius $r$, until $r=a$ is reached. At radius $r$, the inhomogeneous spherical particle with space-dependent dielectric gradation profiles $\epsilon(r)$ and $\chi(r)$ can be replaced by a homogenous sphere with the equivalent dielectric properties $\bar{\epsilon}(r)$ and $\bar{\chi}(r)$. Here the homogeneous sphere should induce the same dipole moment as the original inhomogeneous sphere.

Next, we add to the sphere a spherical shell of infinitesimal thickness $\mathrm{d} r$, with dielectric constant $\epsilon(r)$ and nonlinear susceptibility $\chi(r)$. In this sense, the coated inclusions is composed of a spherical core with radius $r$, linear dielectric constant $\bar{\epsilon}(r)$ and nonlinear susceptibility $\bar{\chi}(r)$, and a shell with outermost radius $r+\mathrm{d} r$, linear dielectric constant $\epsilon(r)$ and nonlinear susceptibility $\chi(r)$. Since these coated inclusions with a very small volume fraction are randomly embedded in a linear host medium, under the quasi-static approximation, we can readily obtain the linear electric potentials in the core, shell and host medium by solving the Laplace equation [76]

$$
\begin{aligned}
& \phi_{c}=-E_{0} A R \cos \theta, \quad R<r, \\
& \phi_{s}=-E_{0}\left(B R-\frac{C r^{3}}{R^{2}}\right) \cos \theta, \quad r<R<r+\mathrm{d} r, \\
& \phi_{h}=-E_{0}\left(R-\frac{D(r+\mathrm{d} r)^{3}}{R^{2}}\right) \cos \theta, \quad R>r+\mathrm{d} r,
\end{aligned}
$$

where

$$
\begin{aligned}
& A=\frac{9 \epsilon_{2} \epsilon(r)}{Q}, \quad B=\frac{3 \epsilon_{2}[\bar{\epsilon}(r)+2 \epsilon(r)]}{Q}, \quad C=\frac{3 \epsilon_{2}[\bar{\epsilon}(r)-\epsilon(r)]}{Q}, \\
& D=\frac{\left[\epsilon(r)-\epsilon_{2}\right][\bar{\epsilon}(r)+2 \epsilon(r)]+\lambda\left[\epsilon_{2}+2 \epsilon(r)\right][\bar{\epsilon}(r)-\epsilon(r)]}{Q},
\end{aligned}
$$

with interfacial parameter $\lambda \equiv[r /(r+\mathrm{d} r)]^{3}$, and

$$
Q=\left[\epsilon(r)+2 \epsilon_{2}\right][\bar{\epsilon}(r)+2 \epsilon(r)]+2 \lambda\left[\epsilon(r)-\epsilon_{2}\right][\bar{\epsilon}(r)-\epsilon(r)] .
$$


The effective (overall) linear dielectric constant of the system is determined by the dilute-limit expression [36]

$$
\epsilon_{e}=\epsilon_{2}+3 p \epsilon_{2} D
$$

where $p$ is the volume fraction of graded particles with radius $r$. The equivalent dielectric constant $\bar{\epsilon}(r+\mathrm{d} r)$ for the graded particles with radius $r+\mathrm{d} r$ can be obtained self-consistently by the vanishing of the dipole factor $D$ by replacing $\epsilon_{2}$ with $\bar{\epsilon}(r+\mathrm{d} r)$. Taking the limit $\mathrm{d} r \rightarrow 0$ and keeping to the first order in $\mathrm{d} r$, we obtain

$$
\begin{aligned}
\bar{\epsilon}(r+\mathrm{d} r) & =\epsilon(r)+3 \epsilon(r) \lambda \cdot \frac{\bar{\epsilon}(r)-\epsilon(r)}{\bar{\epsilon}(r)(1-\lambda)+\epsilon(r)(2+\lambda)} \\
& =\bar{\epsilon}(r)-\frac{\bar{\epsilon}(r)-\epsilon(r)}{r} \cdot\left[3+\frac{\bar{\epsilon}(r)-\epsilon(r)}{\epsilon(r)}\right] \mathrm{d} r .
\end{aligned}
$$

Thus, we have the differential equation for the equivalent dielectric constant $\bar{\epsilon}(r)$ as [112]

$$
\frac{\mathrm{d} \bar{\epsilon}(r)}{\mathrm{d} r}=\frac{[\epsilon(r)-\bar{\epsilon}(r)] \cdot[\bar{\epsilon}(r)+2 \epsilon(r)]}{r \epsilon(r)} .
$$

Note that Eq. (116) is just the Tartar formula, derived for assemblages of spheres with varying radial and tangential conductivity [39].

Next, we speculate on how to derive the equivalent nonlinear susceptibility $\bar{\chi}(r)$. After applying Eq. (112) to the coated particles with radius $r+\mathrm{d} r$, we have

$$
\bar{\chi}(r+\mathrm{d} r) \frac{\left\langle|\mathbf{E}|^{2} \mathbf{E}^{2}\right\rangle_{R \leqslant r+\mathrm{d} r}}{\left|\mathbf{E}_{0}\right|^{2} \mathbf{E}_{0}^{2}}=\lambda \bar{\chi}(r) \frac{\left\langle|\mathbf{E}|^{2} \mathbf{E}^{2}\right\rangle_{R \leqslant r}}{\left|\mathbf{E}_{0}\right|^{2} \mathbf{E}_{0}^{2}}+(1-\lambda) \frac{\left\langle\chi(r)|\mathbf{E}|^{2} \mathbf{E}^{2}\right\rangle_{r<R \leqslant r+\mathrm{d} r}}{\left|\mathbf{E}_{0}\right|^{2} \mathbf{E}_{0}^{2}} .
$$

As $\mathrm{d} r \rightarrow 0$, the left-hand side of the above equation admits

$$
\begin{aligned}
\bar{\chi}(r+\mathrm{d} r) \frac{\left\langle|\mathbf{E}|^{2} \mathbf{E}^{2}\right\rangle_{R \leqslant r+\mathrm{d} r}}{\left|\mathbf{E}_{0}\right|^{2} \mathbf{E}_{0}^{2}}= & \bar{\chi}(r+\mathrm{d} r)\left|\frac{3 \epsilon_{2}}{\bar{\epsilon}(r+\mathrm{d} r)+2 \epsilon_{2}}\right|^{2}\left(\frac{3 \epsilon_{2}}{\bar{\epsilon}(r+\mathrm{d} r)+2 \epsilon_{2}}\right)^{2} \\
= & \bar{\chi}(r)|K|^{2} K^{2}-\mathrm{d} r \bar{\chi}(r)|K|^{2} K^{2}\left[\frac{3 \mathrm{~d} \bar{\epsilon}(r) / \mathrm{d} r}{2 \epsilon_{2}+\bar{\epsilon}(r)}+\left(\frac{\mathrm{d} \bar{\epsilon}(r) / \mathrm{d} r}{2 \epsilon_{2}+\bar{\epsilon}(r)}\right)^{*}\right] \\
& +|K|^{2} K^{2} \frac{\mathrm{d} \bar{\chi}(r)}{\mathrm{d} r} \mathrm{~d} r
\end{aligned}
$$

with $K=\left(3 \epsilon_{2}\right) /\left[\bar{\epsilon}(r)+2 \epsilon_{2}\right]$. The first part of the right-hand side of Eq. (117) is written as

$$
\lambda \frac{\bar{\chi}(r)\left\langle|\mathbf{E}|^{2} \mathbf{E}^{2}\right\rangle_{R \leqslant r}}{\left|\mathbf{E}_{0}\right|^{2} \mathbf{E}_{0}^{2}}=\bar{\chi}(r)|K|^{2} K^{2}\left[1+\left(6 y+2 y^{*}-3\right) \frac{\mathrm{d} r}{r}\right],
$$

where

$$
y=\frac{\left[\epsilon(r)-\epsilon_{2}\right][\bar{\epsilon}(r)-\epsilon(r)]}{\epsilon(r)\left[\bar{\epsilon}(r)+2 \epsilon_{2}\right]} .
$$

The second part of the right-hand side of Eq. (117) has the form [36]

$$
(1-\lambda) \frac{\left\langle\chi(r)|\mathbf{E}|^{2} \mathbf{E}^{2}\right\rangle_{r<R \leqslant r+\mathrm{d} r}}{\left|\mathbf{E}_{0}\right|^{2} \mathbf{E}_{0}^{2}}=\frac{3 \chi(r)}{5 r} \mathrm{~d} r|z|^{2} z^{2}\left(5+18 x^{2}+18|x|^{2}+4 x^{3}+12 x|x|^{2}+24|x|^{2} x^{2}\right),
$$

where

$$
x=\frac{\bar{\epsilon}(r)-\epsilon(r)}{\bar{\epsilon}(r)+2 \epsilon(r)} \quad \text { and } \quad z=\frac{\epsilon_{2}[\bar{\epsilon}(r)+2 \epsilon(r)]}{\epsilon(r)\left[\bar{\epsilon}(r)+2 \epsilon_{2}\right]} .
$$


Substituting Eqs. (118), (119) and (120) into Eq. (117), we have a differential equation for the equivalent nonlinear susceptibility $\bar{\chi}(r)$, namely,

$$
\begin{aligned}
\frac{\mathrm{d} \bar{\chi}(r)}{\mathrm{d} r}= & \bar{\chi}(r)\left[\frac{3 \mathrm{~d} \bar{\epsilon}(r) / \mathrm{d} r}{2 \epsilon_{2}+\bar{\epsilon}(r)}+\left(\frac{\mathrm{d} \bar{\epsilon}(r) / \mathrm{d} r}{2 \epsilon_{2}+\bar{\epsilon}(r)}\right)^{*}\right]+\bar{\chi}(r) \cdot \frac{6 y+2 y^{*}-3}{r}+\frac{3 \chi(r)}{5 r} \cdot\left|\frac{\bar{\epsilon}(r)+2 \epsilon(r)}{3 \epsilon(r)}\right|^{2} \\
& \times\left(\frac{\bar{\epsilon}(r)+2 \epsilon(r)}{3 \epsilon(r)}\right)^{2}\left(5+18 x^{2}+18|x|^{2}+4 x^{3}+12 x|x|^{2}+24|x|^{2} x^{2}\right) .
\end{aligned}
$$

So far, the equivalent $\bar{\epsilon}(r)$ and $\bar{\chi}(r)$ of graded spherical particles of radius $r$ can be calculated, at least numerically, by solving the differential equations Eqs. (116) and (121), as long as $\epsilon(r)$ (dielectric-constant gradation profile) and $\chi(r)$ (nonlinear-susceptibility gradation profile) are given. Here we would like to mention that, even though $\chi(r)$ is independent of $r$, the equivalent $\bar{\chi}(r)$ should still be dependent on $r$ because of $\epsilon(r)$ as a function of $r$. Moreover, for both $\epsilon(r)=\epsilon_{1}$ and $\chi(r)=\chi_{1}$ (i.e., they are both constant and independent of $r$ ), Eqs. (116) and (121) will naturally reduce to the solutions $\bar{\epsilon}(r)=\epsilon_{1}$ and $\bar{\chi}(r)=\chi_{1}$.

To obtain $\bar{\epsilon}(r=a)$ and $\bar{\chi}(r=a)$, we integrate Eqs. (116) and (121) numerically at given initial conditions $\bar{\epsilon}(r \rightarrow 0)$ and $\bar{\chi}(r \rightarrow 0)$. Once $\bar{\epsilon}(r=a)$ and $\bar{\chi}(r=a)$ are calculated, we can take one step forward to work out the effective linear and nonlinear responses $\epsilon_{e}$ and $\chi_{e}$ of the whole composite in the dilute limit, i.e. [74],

$$
\epsilon_{e}=\epsilon_{2}+3 \epsilon_{2} f \frac{\bar{\epsilon}(r=a)-\epsilon_{2}}{\bar{\epsilon}(r=a)+2 \epsilon_{2}},
$$

and

$$
\chi_{e}=f \bar{\chi}(r=a)\left|\frac{3 \epsilon_{2}}{\bar{\epsilon}(r=a)+2 \epsilon_{2}}\right|^{2}\left(\frac{3 \epsilon_{2}}{\bar{\epsilon}(r=a)+2 \epsilon_{2}}\right)^{2} .
$$

\subsubsection{Exact solution for power-law gradation profiles}

Based on the first-principles approach, we have found that, for a power-law dielectric gradation profile, i.e., $\epsilon(r)=$ $A(r / a)^{n}$, the potential in the graded inclusions and the host medium can be exactly given by [113]

$$
\begin{aligned}
& \phi_{i}(r)=-\xi_{1} E_{0} r^{s} \cos \theta, \quad r<a, \\
& \phi_{h}(r)=-E_{0} r \cos \theta+\frac{\xi_{2}}{r^{2}} E_{0} \cos \theta, \quad r>a,
\end{aligned}
$$

where the coefficients $\xi_{1}$ and $\xi_{2}$ have the form

$$
\xi_{1}=\frac{3 a^{1-s} \epsilon_{2}}{s A+2 \epsilon_{2}} \quad \text { and } \quad \xi_{2}=\frac{s A-\epsilon_{2}}{s A+2 \epsilon_{2}} a^{3}
$$

and $s$ is given by

$$
s=\frac{1}{2}\left[\sqrt{9+2 n+n^{2}}-(1+n)\right] .
$$

The local electric field inside the graded inclusions can be derived from the potential $\mathbf{E}=-\nabla \phi$,

$$
\begin{aligned}
\mathbf{E}_{i}= & \xi_{1} E_{0} r^{s-1}\left(s \cos \theta \mathbf{e}_{r}-\sin \theta \mathbf{e}_{\theta}\right)=\xi_{1} E_{0} r^{s-1}\left\{(s-1) \cos \theta \sin \theta \cos \phi \mathbf{e}_{x}\right. \\
& \left.+(s-1) \cos \theta \sin \theta \sin \phi \mathbf{e}_{y}+\left[(s-1) \cos ^{2} \theta+1\right] \mathbf{e}_{z}\right\},
\end{aligned}
$$

where $\mathbf{e}_{r}, \mathbf{e}_{\theta}$, and $\mathbf{e}_{x}, \mathbf{e}_{y}$ and $\mathbf{e}_{z}$ are unix vectors in spherical coordinates and in Cartesian coordinates. In the dilute limit, from Eq. (111), we can obtain the effective linear dielectric constant as follows

$$
\begin{aligned}
\epsilon_{e} & =\epsilon_{2}+\frac{1}{V E_{0}} \int_{\Omega_{i}}\left[A(r / a)^{n}-\epsilon_{2}\right] \mathbf{e}_{z} \cdot \mathbf{E}_{i} \mathrm{~d} V \\
& =\epsilon_{2}+3 \epsilon_{2} f \frac{2+s}{s A+2 \epsilon_{2}}\left(\frac{A}{2+n+s}-\frac{\epsilon_{2}}{2+s}\right) .
\end{aligned}
$$




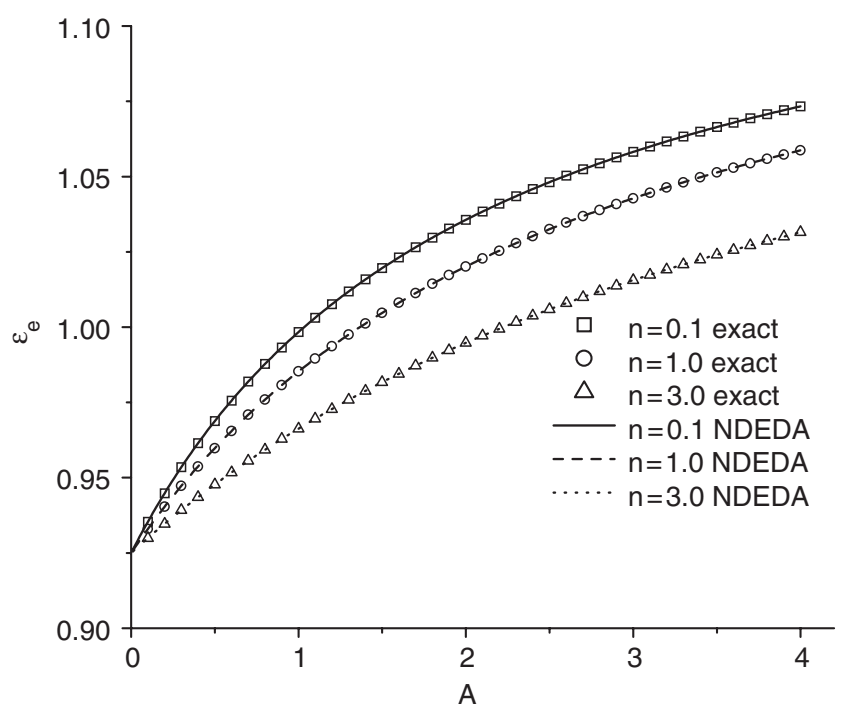

Fig. 33. The effective linear dielectric constant $\epsilon_{e}$ versus $A$ for the power-law dielectric gradation profile $\epsilon(r)=A(r / a)^{n}$ in the dilute limit $f=0.05$ [20]. Lines: numerical results from the NDEDA [Eq. (116)]; Symbols: exact results [Eq. (126)].

On the other hand, the substitution of Eq. (125) into Eq. (112) yields

$$
\begin{aligned}
\chi_{e} & =\frac{1}{V} \int_{\Omega_{i}} \chi(r)\left|\xi_{1}\right|^{2} \xi_{1}^{2}\left(s^{2} \cos ^{2} \theta+\sin ^{2} \theta\right)^{2} r^{4 s-2} \sin \theta \mathrm{d} r \mathrm{~d} \theta \mathrm{d} \phi \\
& =\frac{f}{5 a^{3}}\left|\xi_{1}\right|^{2} \xi_{1}^{2}\left(8+4 s+3 s^{4}\right) \cdot \int_{0}^{a} \chi(r) r^{4 s-2} \mathrm{~d} r .
\end{aligned}
$$

For example, for a linear profile of $\chi(r)$, i.e., $\chi(r)=k_{1}+k_{2} \cdot r / a$, Eq. (127) leads to

$$
\chi_{e}=\frac{f}{20}\left|\frac{3 \epsilon_{2}}{s A+2 \epsilon_{2}}\right|^{2}\left(\frac{3 \epsilon_{2}}{s A+2 \epsilon_{2}}\right)^{2}\left(8+4 s^{2}+3 s^{4}\right)\left(\frac{k_{2}}{s}+\frac{4 k_{1}}{4 s-1}\right) .
$$

In addition, for a power-law profile of $\chi(r)$, namely, $\chi(r)=k_{1}(r / a)^{k_{2}}$, Eq. (127) produces

$$
\chi_{e}=\frac{f}{5}\left|\frac{3 \epsilon_{2}}{s A+2 \epsilon_{2}}\right|^{2}\left(\frac{3 \epsilon_{2}}{s A+2 \epsilon_{2}}\right)^{2} k_{1}\left(\frac{8+4 s^{2}+3 s^{4}}{k_{2}-1+4 s}\right) .
$$

We are now in a position to evaluate the NDEDA. For the comparison between the first-principles approach and the NDEDA, we first perform numerical calculations for the case where the dielectric constant exhibits power-law gradation profiles $\epsilon(r)=A(r / a)^{n}$, while the third-order nonlinear susceptibility shows two model gradation profiles: (a) linear profile $\chi(r)=k_{1}+k_{2} \cdot r / a$, and (b) power-law profile $\chi(r)=k_{1}(r / a)^{k_{2}}$. Without loss of generality, we take $\epsilon_{2}=1$ and $a=1$ for numerical calculations. The fourth-order Runge-Kutta algorithm is adopted to integrate the differential equations [Eqs. (116) and (121)] with step size 0.01. Meanwhile, the initial core radius is set to be 0.001 . It was verified that this step size guarantees accurate numerics.

In Fig. 33, the effective linear dielectric constant $\left(\epsilon_{e}\right)$ is plotted as a function of $A$ for various indices $n$. It is shown that $\epsilon_{e}$ exhibits a monotonic increase for increasing $A$ (and decreasing $n$ ). This can be understood by using the equivalent dielectric constant $\bar{\epsilon}(r=a)$ which increases as $A$ increases ( $n$ decreases). Moreover, the excellent agreement between the NDEDA [Eq. (116)] and the first-principles approach [Eq. (126)] is shown as well.

Next, the effective third-order nonlinear susceptibility $\left(\chi_{e}\right)$ is plotted as a function of $A$ for the linear gradation profile $\chi(r)=k_{1}+k_{2} \cdot r / a$ (Fig. 34), and for the power-law profile $\chi(r)=k_{1}(r / a)^{k_{2}}$ (Fig. 35). We find that the effective nonlinear susceptibility decreases for increasing $A$. The reason is that, as mentioned above, for larger $A$, the graded inclusions possess larger equivalent dielectric constant, and the local field inside the nonlinear inclusions will become 

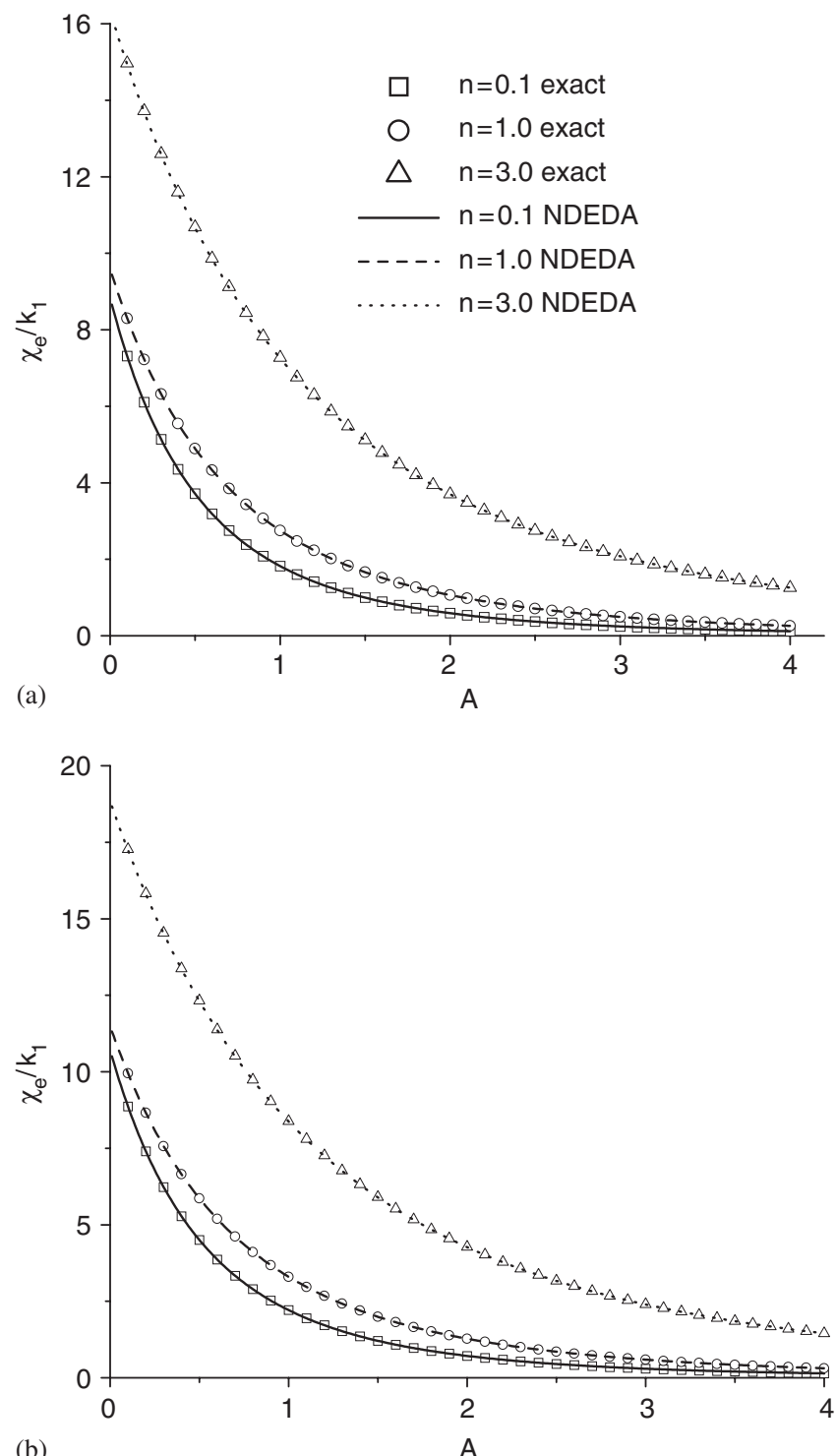

(b)

A

Fig. 34. The effective third-order nonlinear susceptibility $\chi_{e}$ versus $A$ for power-law dielectric-constant gradation profile $\epsilon(r)=A(r / a)^{n}$ and linear nonlinear-susceptibility gradation profile $\chi(r)=k_{1}+k_{2} \cdot r / a$ with (a) $k_{1}=1$ and $k_{2}=1$, and (b) $k_{1}=2$ and $k_{2}=3$ [20]. Lines: numerical results from the NDEDA [Eqs. (116) and (121)]; Symbols: exact results [Eq. (127)].

more weak, which results in a weaker effective nonlinear susceptibility $\left(\chi_{e}\right)$. In addition, increasing $n$ leads generally to increasing $\chi_{e}$, and such a trend is clearly observed at large $A$. Again, we obtain the excellent agreement between the first-principles approach [Eqs. (128) and (129)] and the NDEDA [Eqs. (116) and (121)].

In what follows, we investigate the surface plasmon resonance effect on the metal-dielectric composite. We adopt the Drude-like dielectric constant for graded metal particles, namely,

$$
\epsilon(r)=1-\frac{\omega_{p}^{2}(r)}{\omega[\omega+\mathrm{i} \gamma(r)]},
$$

where $\omega_{p}(r)$ and $\gamma(r)$ are the radius-dependent plasma frequency and damping coefficient, respectively. For the sake of simplicity, set $\chi(r)=\chi_{1}$ to be independent of $r$, in an attempt to emphasize the enhancement of the effective optical 


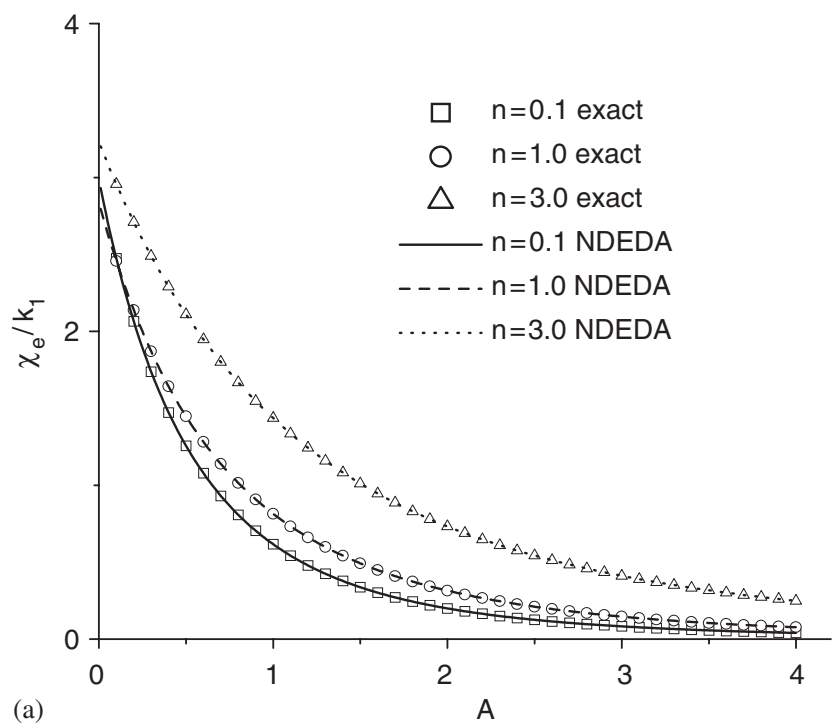

(a)

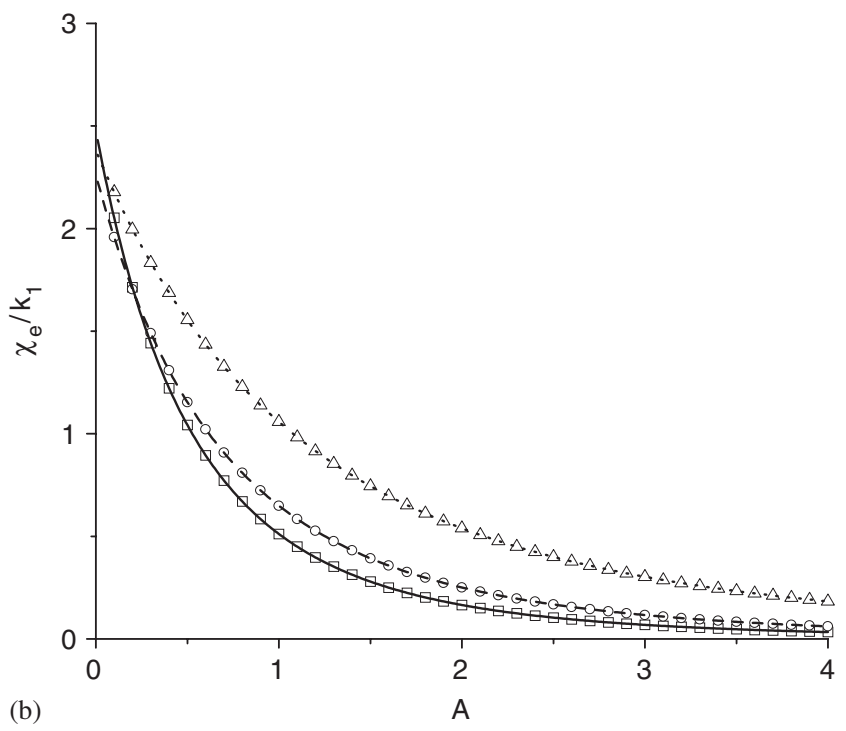

Fig. 35. Same as Fig. 34, but for power-law nonlinear-susceptibility gradation profile $\chi(r)=k_{1}(r / a)^{k_{2}}$ [20].

nonlinearity, and $\epsilon_{2}=1.77$ (the dielectric constant of water). We assume further $\omega_{p}(r)$ to be

$$
\omega_{p}(r)=\omega_{p}\left(1-k_{\omega} \cdot \frac{r}{a}\right), \quad r<a .
$$

This form is quite physical for $k_{\omega}>0$, since the center of grains can be better metallic so that $\omega_{p}(r)$ is larger, while the boundary of the grain may be poorer metallic so that $\omega_{p}(r)$ is much smaller. Such the variation can also appear because of the temperature effect [114]. For small particles, we have the radius-dependent $\gamma(r)$ as [80]

$$
\gamma(r)=\gamma(\infty)+\frac{k_{\gamma}}{r / a}, \quad r<a,
$$

where $\gamma(\infty)$ stands for the damping coefficient in the bulk material. Here $k_{\gamma}$ is a constant which is related to the Fermi velocity $v_{\mathrm{F}}$. In this case, the exact solution being predicted by a first-principles approach is absent. Fortunately, we can resort to the NDEDA instead. 

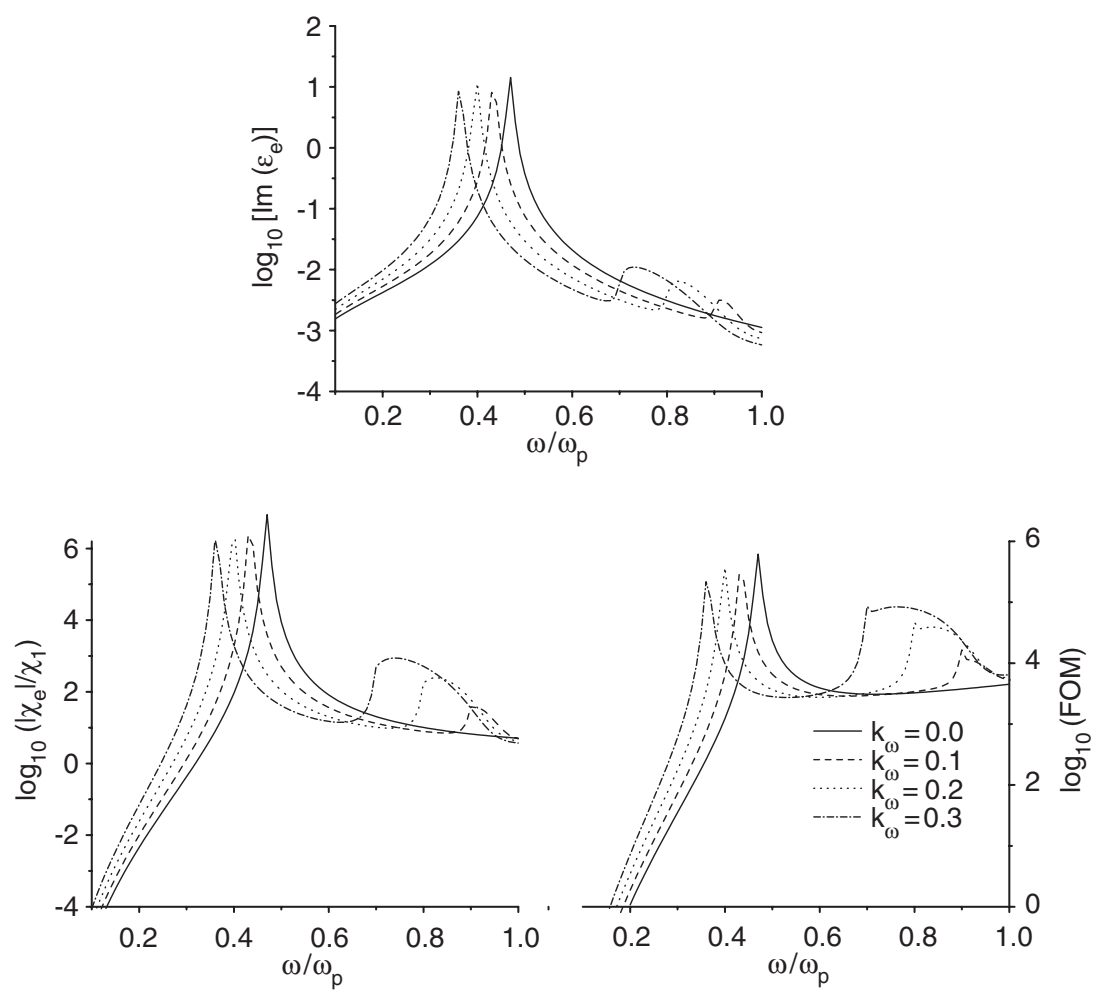

Fig. 36. (a) The linear optical absorption $\operatorname{Im}\left(\epsilon_{e}\right)$, (b) the enhancement of the third-order optical nonlinearity $\left|\chi_{e}\right| / \chi_{1}$, and (c) the figure of merit $\equiv\left|\chi_{e}\right| / \operatorname{Im}\left(\epsilon_{e}\right)$ versus the incident angular frequency $\omega / \omega_{p}$ for dielectric-constant gradation profile $\epsilon(r)=1-\omega_{p}^{2}(r) /[\omega(\omega+\mathrm{i} \gamma(r))]$ with $\omega_{p}(r)=\omega_{p}\left(1-k_{\omega} \cdot r / a\right)$ and $\gamma(r)=0.01 \omega_{p}[20]$. Parameters: $\epsilon_{2}=1.77$ and $f=0.05$.

In Fig. 36, we plot the optical absorption $\left[\sim \operatorname{Im}\left(\epsilon_{e}\right)\right]$, the modulus of the effective third-order optical nonlinearity enhancement $\left(\left|\chi_{e}\right| / \chi_{1}\right)$ and the figure of merit $\left(\left|\chi_{e}\right| / \operatorname{Im}\left(\epsilon_{e}\right)\right)$ versus the incident angular frequency $\omega$. For the case of the homogeneous particles, i.e., $k_{\omega}=0$, there is a single sharp peak at $\omega \approx 0.5 \omega_{p}$, corresponding to the surface plasmon resonance, as expected. However, for the case of the graded particles, i.e. $k_{\omega} \neq 0$, besides a sharp peak, a broad continuous resonant band in the high-frequency region is apparently observed. The position of the sharp peak can be estimated from the resonant condition $\operatorname{Re}[\bar{\epsilon}(r=a)]+2 \epsilon_{2}=0$, while the broad continuous spectrum is indeed a salient result of the gradation profile. More exactly, the broad spectrum results from the effect of the radius-dependent plasma frequency. In Ref. [78], we found that, when the shell model is taken into account, a broad continuous spectrum should be expected to occur around the large pole in the spectral density function. In fact, the graded particles under consideration can be regarded as a certain limit of multi shells, which thus should yield the broader spectra in $\operatorname{Im}\left(\epsilon_{e}\right)$, $\left|\chi_{e}\right| / \chi_{1}$ as well as $\left|\chi_{e}\right| / \operatorname{Im}\left(\epsilon_{e}\right)$. In addition, we note that increasing $k_{\omega}$ makes both the surface plasmon frequency and the center of the resonant bands red-shifted. In particular, the resonant bands can become more broad due to strong inhomogeneity of the particles. From the figure, we conclude that, although the third-order optical nonlinearity is always accompanied with the optical absorption, the figure of merit in the high frequency region is still attractive due to the presence of weak optical absorption. Thus, we believe that graded particles have potential applications in obtaining the optimal figure of merit, and make the composite media more realistic for practical applications.

Finally, we focus on the effect of $\gamma(r)$ on the nonlinear optical property in Fig. 37. As evident from the results, the variation of $k_{\gamma}$ plays an important role in the magnitude of the effective optical properties, particularly at the surface plasmon resonance frequency.

We have developed an NDEDA (nonlinear differential effective dipole approximation) to calculate the effective linear and nonlinear dielectric responses of composite media containing nonlinear graded inclusions. The results obtained from the NDEDA are compared with the exact solutions derived from a first-principles approach for the power-law 

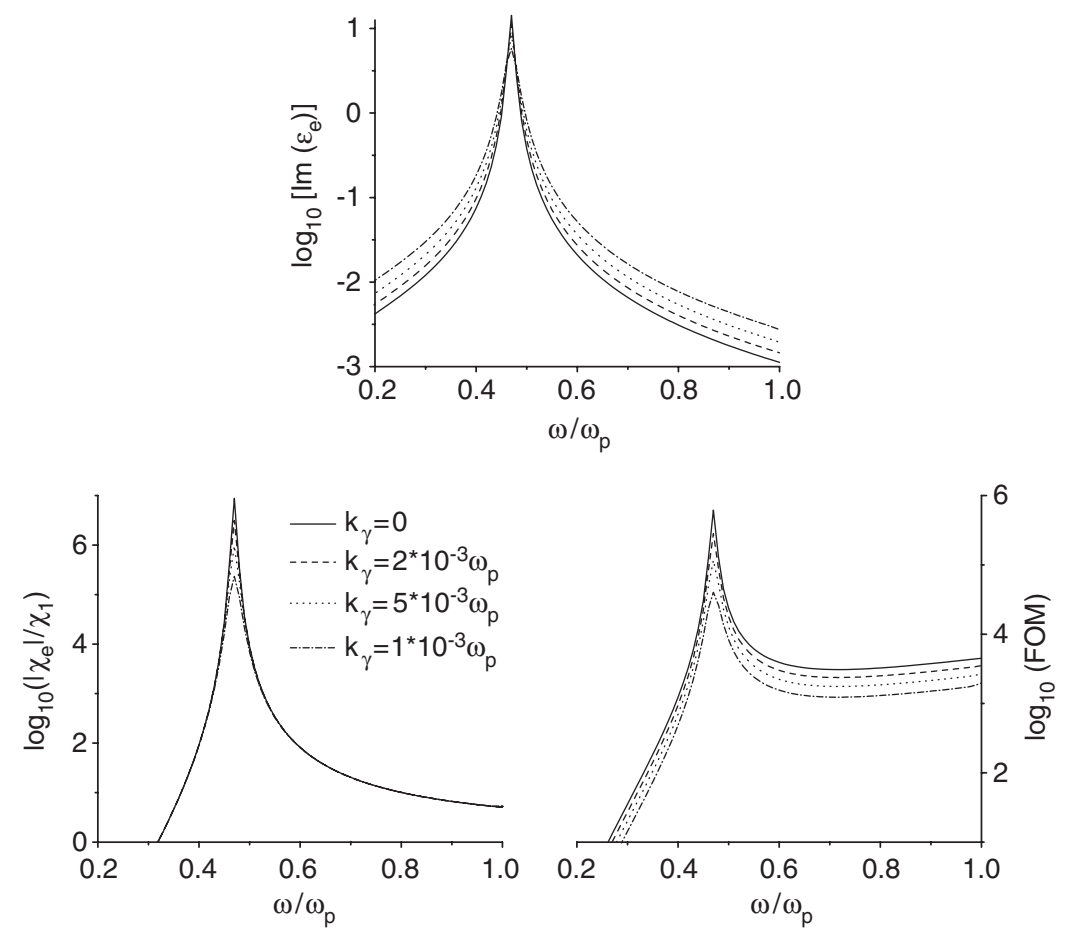

Fig. 37. Same as Fig. 36, but with $\omega_{p}(r)=\omega_{p}$ and $\gamma(r)=\gamma(\infty)+k_{\gamma} /(r / a)$ for $\gamma(\infty)=0.01 \omega_{p}$ [20].

dielectric gradation profiles, and the excellent agreement between them has been shown. We should remark that the exact solutions are also obtainable for the linear dielectric gradation profiles with small slopes (the derivation not shown here). In this case, the excellent agreement between the two methods can be shown as well since the NDEDA is valid indeed for arbitrary gradation profiles. In general, the exact solution is quite few in realistic composite research, and thus our NDEDA can be used as a benchmark.

The NDEDA was derived for the composite containing the nonlinear graded inclusions in a linear host. Interestingly, it can be readily generalized to the composite system where the graded inclusions and the host are both nonlinear [4]. In this situation, the effective third-order nonlinear response can be written as [11,79],

$$
\begin{aligned}
\chi_{e}= & f \bar{\chi}(r=a)\left|\frac{3 \epsilon_{2}}{\bar{\epsilon}(r=a)+2 \epsilon_{2}}\right|^{2}\left(\frac{3 \epsilon_{2}}{\bar{\epsilon}(r=a)+2 \epsilon_{2}}\right)^{2}+\chi_{2}(1-f) \\
& +\chi_{2} f\left(3 \beta+\beta^{*}+\frac{18}{5} \beta^{2}+\frac{18}{5}|\beta|^{2}+\frac{6}{5}|\beta|^{2} \beta+\frac{2}{5} \beta^{3}+\frac{8}{5}|\beta|^{2} \beta^{2}\right),
\end{aligned}
$$

where $\beta \equiv\left[\bar{\epsilon}(r=a)-\epsilon_{2}\right] /\left[\bar{\epsilon}(r=a)+2 \epsilon_{2}\right]$ and $\chi_{2}$ is the third-order nonlinear susceptibility of the host medium. As a matter of fact, for this purpose, the perturbation method can also be adopted [115].

The NDEDA is strictly valid in the dilute limit. To achieve the strong optical nonlinearity enhancement, we need possibly nonlinear inclusions with high volume fractions. In this connection, the effect of the volume fraction is expected to cause a further broadening of the resonant peak, and possibly, a desired separation of the optical absorption peak from the nonlinearity enhancement due to mutual interactions [7]. Therefore, it is of particular interest to generalize the NDEDA for treating the case of high volume fractions.

It is also instructive to develop the first-principles approach to weakly nonlinear graded composites. The perturbation approach [67] in weakly nonlinear composites is just suitable for this problem. Moreover, with the aim of the variational approach [116], the NDEDA may be applied to the cases of strong nonlinearity, where the linear part [ $\epsilon(r)]$ in Eq. (109) vanishes. On the other hand, based on the self-consistent mean-field approximation [117], the applicability of NDEDA to more general cases, where linear $[\epsilon(r)]$ and nonlinear $\left[\chi(r)|\mathbf{E}|^{2}\right]$ parts can be comparable, may be also possible. 
To sum up, we put forth an NDEDA and a first-principles approach for investigating the optical responses of nonlinear graded spherical particles. The excellent agreement between the two methods has been shown. As an application, we applied the NDEDA to discuss the surface plasmon resonance effect on the effective linear and nonlinear optical properties like the optical absorption, the optical nonlinearity enhancement, and the figure of merit. It is found that the dielectric gradation profile can be used to control the surface plasmon resonance and achieve the large figure of merit in the high-frequency region, where the optical absorption is quite small.

\subsection{Composites of graded particles with dielectric anisotropy}

The above gradation models were built up under the assumption that the graded inclusions exhibit isotropic dielectric response. However, dielectric anisotropy occurs naturally due to the presence of gradation inside the particles. Moreover, there are many inhomogeneous materials with spatial anisotropy, like polycrystal aggregates of a single anisotropic component [118], liquid crystal droplets [119], and cell membranes containing mobile charges [120]. In these situations, the local dielectric coefficient should be tensorial. Thus, for a better understanding of the dielectric-anisotropy effect, it is necessary to generalize our previous isotropic gradation models [19,110-112] accordingly.

The nonlinearity is a common phenomena in realistic graded materials. And the spatial anisotropy effect has not yet been investigated in the traditional theories. Below we shall develop a new theory, in an attempt to study the effective linear and nonlinear optical properties of composite media, by taking into account the dielectric anisotropy of the nonlinear graded particles. For the dielectric tensor of these graded inclusions, the components of the tensorial dielectric constant of interest will be assumed to vary along the radius of the particles continuously.

\subsubsection{Model and definition of effective linear dielectric constant and third-order nonlinear susceptibility}

Let us consider a dilute composite material, where the identical spherical inclusions having a dielectric constant tensor $\stackrel{\leftrightarrow}{\varepsilon}_{1}$, with radius $a$, are randomly embedded in a linear isotropic host with (scalar) dielectric constant $\epsilon_{2}$. Inside the anisotropic inclusions, the local constitutive relation between the displacement $(\mathbf{D})$ and the electric field $(\mathbf{E})$ is given by [121]

$$
D_{i}=\sum_{j} \epsilon_{i j} E_{j}+\sum_{j k l} \chi_{i j k l} E_{j} E_{k} E_{l}^{*}
$$

Here $D_{i}$ and $E_{i}$ are, respectively, the $i$ th Cartesian components of $\mathbf{D}$ and $\mathbf{E}$. It is worth remarking that $\epsilon_{i j}$ and $\chi_{i j k l}$ are the second-rank and fourth-rank Cartesian tensors, respectively. Our analysis will be limited to the case of weak nonlinearity. In other words, the nonlinear part in Eq. (134) will be assumed to be small when compared with the linear part.

In what follows, the dielectric tensor for the the anisotropic spherical inclusions is assumed to be diagonal in spherical coordinates, with a value $\epsilon_{1 t}(r)$ in the tangential directions and $\epsilon_{1 r}(r)$ in the radial direction [25,120,122]. Here, both dielectric gradation profiles $\epsilon_{1 r}(r)$ and $\epsilon_{1 t}(r)$ will be mathematically represented as radial functions [25]. In view of the spherical symmetry, we can express the dielectric constant tensor $\stackrel{\leftrightarrow}{\varepsilon}_{1}(r)$ of graded particles in the form

$$
\overleftrightarrow{\varepsilon}_{1}(r)=\left(\begin{array}{ccc}
\varepsilon_{1 r}(r) & 0 & 0 \\
0 & \epsilon_{1 t}(r) & 0 \\
0 & 0 & \varepsilon_{1 t}(r)
\end{array}\right) .
$$

Note the above form is in spherical coordinates, rather than in Cartesian coordinates. Nevertheless, it can also be represented in Cartesian coordinates by a transformation using appropriate rotation matrices.

As the graded inclusions with dielectric anisotropy are randomly oriented, the whole sample should be macroscopically isotropic. Thus, we can define the effective linear dielectric constant $\epsilon_{e}$ and the third-order nonlinear susceptibility $\chi_{e}$ of the whole composite as $[74,121]$

$$
\mathbf{D}=\epsilon_{e} \mathbf{E}_{0}+\chi_{e}\left|\mathbf{E}_{0}\right|^{2} \mathbf{E}_{0},
$$


where $\langle\cdots\rangle$ stands for the spatial average, and $\mathbf{E}_{0}=E_{0} \mathbf{e}_{z}$ denotes the external applied field along $z$-axis. In Eq. (136), the effective linear dielectric constant $\epsilon_{e}$ is given by

$$
\epsilon_{e} \mathbf{E}_{0}=\frac{1}{V} \int_{V} \stackrel{\leftrightarrow}{\varepsilon} \mathbf{E}_{\text {lin }} \mathrm{d} V=f\left\langle\stackrel{\leftrightarrow}{\varepsilon}{ }_{1} \cdot \mathbf{E}_{1, \operatorname{lin}}\right\rangle+(1-f) \epsilon_{2}\left\langle\mathbf{E}_{2, \operatorname{lin}}\right\rangle
$$

where $f$ is the volume fraction of the graded inclusions. Here, the subscript 'lin' denotes the linear local field inside the graded inclusions or the host.

In view of the existence of nonlinearity inside the anisotropic graded particles, $\chi_{e}$ is given by $[74,123]$

$$
\chi_{e} E_{0}^{4}=\frac{1}{V} \sum_{i j k l} \int_{V} \chi_{i j k l} E_{\operatorname{lin}, i} E_{\operatorname{lin}, j} E_{\operatorname{lin}, k} E_{\operatorname{lin}, l}^{*} \mathrm{~d} V=f \sum_{i j k l}\left\langle\chi_{i j k l} E_{\operatorname{lin}, i} E_{\operatorname{lin}, j} E_{\operatorname{lin}, k} E_{\operatorname{lin}, l}\right\rangle
$$

Here $E_{\text {lin }, i}$ denotes the Cartesian component of the linear local electric field. Then, just as in isotropic composites [74], both $\epsilon_{e}$ and $\chi_{e}$ in nonlinear composite media with local dielectric anisotropy can be expressed (to the lowest order in the nonlinearity) in terms of the electric field in the related linear medium as well.

In the next section, we will develop an NADEDA (nonlinear anisotropic differential effective dipole approximation), so as to derive the equivalent linear dielectric constant $\bar{\epsilon}(a)$ and third-order nonlinear susceptibility $\bar{\chi}(a)$ of the nonlinear graded inclusions. In this connection, $\epsilon_{e}$ and $\chi_{e}$ of this anisotropic graded composite media can further be derived in the dilute limit.

\subsubsection{Nonlinear anisotropic differential effective dipole approximation}

To put forth an NADEDA (nonlinear anisotropic differential effective dipole approximation) for graded particles with dielectric anisotropy, we regard the gradation profiles as a multi-shell construction. In detail, we build up the dielectric profile by adding shells gradually [112]. Let us start with an infinitesimal spherical core with linear dielectric constants $\epsilon_{1 r}(r=0)=\epsilon_{1 t}(r=0)=\epsilon(0)$ and nonlinear susceptibility $\chi_{i j k l}$, and keep on adding shells with the tangential and radial dielectric constant $\epsilon_{1 t}(r)$ and $\epsilon_{1 t}(r)$, and the Cartesian fourth-rank tensorial nonlinear susceptibility $\chi_{i j k l}$ (to show the optical nonlinearity enhancement, we always assume $\chi_{i j k l}$ to be independent of $r$ ), at radius $r$, until $r=a$ is reached.

At radius $r$, we have an inhomogeneous spherical particle with spatially varying dielectric constant, which are characterized by the gradation profiles $\epsilon_{1 r}(r)$ and $\epsilon_{1 t}(r)$, and with tensorial nonlinear susceptibility $\chi_{i j k l}$. Then, we can regard such an inhomogeneous particle as an effective homogeneous one with the equivalent isotropic dielectric properties $\bar{\epsilon}(r)$ and $\bar{\chi}(r)$. Here the homogeneous sphere should induce the same dipole moment as the original inhomogeneous sphere. Then, we add to the homogeneous particle a spherical shell of infinitesimal thickness $\mathrm{d} r$, with linear dielectric constants, $\epsilon_{1 r}(r)$ and $\epsilon_{1 t}(r)$, and nonlinear susceptibility, $\chi_{i j k l}$. In this situation, the coated inclusions are composed of a spherical core with radius $r$, linear dielectric constant $\bar{\epsilon}(r)$ as well as nonlinear susceptibility $\bar{\chi}(r)$, and a shell with outermost radius $r+\mathrm{d} r$, linear dielectric constants $\epsilon_{1 r}(r)$ and $\epsilon_{1 t}(r)$, as well as nonlinear susceptibility $\chi_{i j k l}$.

For the graded particles with dielectric anisotropy describe Eq. (135), the displacement vector is related to the field, $\mathbf{D}=\stackrel{\leftrightarrow}{\varepsilon}_{1}(r) \cdot \mathbf{E}$. In view of $\mathbf{E}=-\nabla \Phi$, we have the following electrostatic equation:

$$
\nabla \cdot\left(\stackrel{\leftrightarrow}{\varepsilon}_{1}(r) \cdot \nabla \Phi\right)=0
$$

In spherical coordinates, Eq. (139) can be cast into

$$
\frac{1}{r^{2}} \frac{\partial}{\partial r}\left(r^{2} \epsilon_{1 r}(r) \frac{\partial \Phi}{\partial r}\right)+\frac{1}{r^{2} \sin \theta} \frac{\partial}{\partial \theta}\left(\sin \theta \epsilon_{1 t}(r) \frac{\partial \Phi}{\partial r}\right)+\frac{1}{r^{2} \sin ^{2} \theta} \frac{\partial}{\partial \psi}\left(\epsilon_{1 t}(r) \frac{\partial \Phi}{\partial \psi}\right)=0 .
$$

Let us consider the composite where the coated inclusions are randomly embedded in the linear host medium. Under the quasi-static approximation, we can readily obtain the linear electric potentials inside the core, shell and host medium 
by solving Eq. (140),

$$
\begin{aligned}
& \phi_{c}=-E_{0} A R \cos \theta, \quad R<r, \\
& \phi_{s}=-E_{0}\left(B(r+\mathrm{d} r)^{1-\delta} R^{\delta}-\frac{C r^{(1+2 \delta)}(r+\mathrm{d} r)^{1-\delta}}{R^{\delta+1}}\right) \cos \theta, \quad r<R<r+\mathrm{d} r, \\
& \phi_{h}=-E_{0}\left(R-\frac{D(r+\mathrm{d} r)^{3}}{R^{2}}\right) \cos \theta, \quad R>r+\mathrm{d} r .
\end{aligned}
$$

Here the four unknown parameters $A, B, C$ and $D$ can be determined by applying the appropriate boundary conditions on the interfaces. As a result, we obtain

$$
\begin{aligned}
& A=\frac{3(1+2 \delta) \epsilon_{2} \epsilon_{1 r}(r) \lambda^{(\delta-1) / 3}}{Q}, \quad B=\frac{3 \epsilon_{2}\left[\bar{\epsilon}(r)+(1+\delta) \epsilon_{1 r}(r)\right]}{Q}, \quad C=\frac{3 \epsilon_{2}\left[\bar{\epsilon}(r)-\delta \cdot \epsilon_{1 r}(r)\right]}{Q}, \\
& D=\frac{\left[\delta \cdot \epsilon_{1 r}(r)-\epsilon_{2}\right]\left[\bar{\epsilon}(r)+(1+\delta) \epsilon_{1 r}(r)\right]+\lambda^{(1+2 \delta) / 3}\left[\epsilon_{2}+(1+\delta) \epsilon_{1 r}(r)\right]\left[\bar{\epsilon}(r)-\delta \cdot \epsilon_{1 r}(r)\right]}{Q},
\end{aligned}
$$

with interfacial parameter $\lambda \equiv[r /(r+\mathrm{d} r)]^{3}$ and $\delta \equiv-1 / 2+\sqrt{1 / 4+2 \epsilon_{1 t}(r) / \epsilon_{1 r}(r)}$, and

$$
Q=\left[\delta \cdot \epsilon_{1 r}(r)+2 \epsilon_{2}\right]\left[\bar{\epsilon}(r)+(1+\delta) \epsilon_{1 r}(r)\right]+\lambda^{(1+2 \delta) / 3}\left[(1+\delta) \epsilon_{1 r}(r)-2 \epsilon_{2}\right]\left[\bar{\epsilon}(r)-\delta \cdot \epsilon_{1 r}(r)\right] .
$$

If $\epsilon_{1 t}(r)=\epsilon_{1 r}(r)$, the physical parameter $\delta=1$, and then Eq. (8) degenerates to the isotropic form.

The effective (overall) linear dielectric constant of the system is determined by the dilute-limit expression [36]

$$
\epsilon_{e}=\epsilon_{2}+3 p \epsilon_{2} D
$$

where $p$ is the volume fraction of the graded particles with radius $r$. The equivalent dielectric constant $\bar{\epsilon}(r+d r)$ for the graded particles with radius $r+\mathrm{d} r$ can be self-consistently obtained by the vanishing of the dipole factor $D$ by replacing $\epsilon_{2}$ with $\bar{\epsilon}(r+\mathrm{d} r)$. Taking the limit $\mathrm{d} r \rightarrow 0$ and keeping to the first order in $\mathrm{d} r$, we obtain

$$
\begin{aligned}
\bar{\epsilon}(r+\mathrm{d} r) & =\epsilon_{1 r}(r)\left\{1+\frac{\bar{\epsilon}(r)\left[(\delta-1)+(\delta+2) \lambda^{(1+2 \delta) / 3}\right]+\epsilon_{1 r}(r)\left[\left(\delta^{2}-1\right)-(\delta+2) \delta \lambda^{(1+2 \delta) / 3}\right]}{\bar{\epsilon}(r)\left(1-\lambda^{(1+2 \delta) / 3}\right)+\epsilon_{1 r}(r)\left[\delta+1+\delta \cdot \lambda^{(1+2 \delta) / 3}\right]}\right\} \\
& =\bar{\epsilon}(r)+\frac{\left[\delta \epsilon_{1 r}(r)-\bar{\epsilon}(r)\right]\left[(\delta+1) \epsilon_{1 r}(r)+\bar{\epsilon}(r)\right]}{r \epsilon_{1 r}(r)} \mathrm{d} r .
\end{aligned}
$$

Thus, we have the differential equation for the equivalent dielectric constant $\bar{\epsilon}(r)$,

$$
\frac{\mathrm{d} \bar{\epsilon}(r)}{\mathrm{d} r}=\frac{\delta(\delta+1)\left[\epsilon_{1 r}(r)\right]^{2}-\bar{\epsilon}(r) \epsilon_{1 r}(r)-[\bar{\epsilon}(r)]^{2}}{r \epsilon_{1 r}(r)} .
$$

Note that Eq. (144) is just the Tartar formula, which was derived for assemblages of spheres with varying radial and tangential conductivity [39]. If $\epsilon_{1 r}$ is independent of $r$, namely $\epsilon_{1 r}=\epsilon_{1}$, we have $\delta=1$ due to isotropic property at $r=0$, and then Eq. (144) predicts $\bar{\epsilon}(r)=\epsilon_{1}$, as expected.

Next, we speculate on how to derive the equivalent nonlinear susceptibility $\bar{\chi}(r)$. After applying Eq. (138) to the coated particles with radius $r+\mathrm{d} r$, we have

$$
\bar{\chi}(r+\mathrm{d} r) \frac{\left\langle|E|^{2} E^{2}\right\rangle_{R \leqslant r+\mathrm{d} r}}{\left|E_{0}\right|^{2} E_{0}^{2}}=\lambda \bar{\chi}(r) \frac{\left\langle|E|^{2} E^{2}\right\rangle_{R \leqslant r}}{\left|E_{0}\right|^{2} E_{0}^{2}}+(1-\lambda) \frac{\sum_{i j k l}\left\langle\chi_{i j k l} E_{i} E_{j} E_{k} E_{l}^{*}\right\rangle_{r<R \leqslant r+\mathrm{d} r}}{\left|E_{0}\right|^{2} E_{0}^{2}} .
$$


As $\mathrm{d} r \rightarrow 0$, the left-hand side of the above equation admits

$$
\begin{aligned}
\bar{\chi}(r+\mathrm{d} r) \frac{\left\langle|E|^{2} E^{2}\right\rangle_{R \leqslant r+\mathrm{d} r}}{\left|E_{0}\right|^{2} E_{0}^{2}}= & \bar{\chi}(r+\mathrm{d} r)\left|\frac{3 \epsilon_{2}}{\overline{\bar{\epsilon}}(r+\mathrm{d} r)+2 \epsilon_{2}}\right|^{2}\left(\frac{3 \epsilon_{2}}{\bar{\epsilon}(r+\mathrm{d} r)+2 \epsilon_{2}}\right)^{2} \\
= & \bar{\chi}(r)|K|^{2} K^{2}-\mathrm{d} r \bar{\chi}(r)|K|^{2} K^{2}\left[\frac{3 \mathrm{~d} \bar{\epsilon}(r) / \mathrm{d} r}{2 \epsilon_{2}+\bar{\epsilon}(r)}+\left(\frac{\mathrm{d} \bar{\epsilon}(r) / \mathrm{d} r}{2 \epsilon_{2}+\bar{\epsilon}(r)}\right)^{*}\right] \\
& +|K|^{2} K^{2} \frac{\mathrm{d} \bar{\chi}(r)}{\mathrm{d} r} \cdot \mathrm{d} r
\end{aligned}
$$

with $K=\left(3 \epsilon_{2}\right) /\left[\bar{\epsilon}(r)+2 \epsilon_{2}\right]$. The first part of the right-hand side of Eq. (145) is written as

$$
\lambda \frac{\bar{\chi}(r)\left\langle|E|^{2} E^{2}\right\rangle_{R \leqslant r}}{\left|E_{0}\right|^{2} E_{0}^{2}}=\bar{\chi}(r)|K|^{2} K^{2}\left[1+\left(3 y+y^{*}-3\right) \frac{\mathrm{d} r}{r}\right],
$$

where

$$
y=\frac{\left[(1+\delta) \epsilon_{1 r}(r)-2 \epsilon_{2}\right]\left[\bar{\epsilon}(r)-\delta \cdot \epsilon_{1 r}(r)\right]}{\epsilon_{1 r}(r)\left[\bar{\epsilon}(r)+2 \epsilon_{2}\right]}-\delta+1 .
$$

The term $U \equiv\left(\sum_{i j k l}\left\langle\chi_{i j k l}(r) E_{i} E_{j} E_{k} E_{l}^{*}\right\rangle_{r<R \leqslant r+\mathrm{d} r}\right) /\left(\left|E_{0}\right|^{2} E_{0}^{2}\right)$ in Eq. (145) is written as

$$
\begin{aligned}
U= & {\left[\left(\chi_{x x y y}+\chi_{y x x y}+\chi_{x y x y}+\chi_{x y y x}+\chi_{y x y x}+\chi_{y y x x}+3 \chi_{x x x x}+3 \chi_{y y y y}\right) U_{p 1}\right.} \\
& \times\left(\chi_{x x z z}+\chi_{x z x z}+\chi_{z x x z}+\chi_{z y y z}+\chi_{y z y z}+\chi_{y y z z}\right) U_{p 2}+\left(\chi_{z z x x}+\chi_{x z z x}\right. \\
& \left.\left.+\chi_{z x z x}+\chi_{y z z y}+\chi_{z y z y}+\chi_{z z y y}\right) U_{p 3}+\chi_{z z z z} U_{p 4}\right] \frac{|K|^{2} K^{2}}{315}
\end{aligned}
$$

where

$$
\begin{aligned}
U_{p 1}= & {\left[B_{2}(\delta-1)+C_{2}(2+\delta)\right]^{3} \cdot\left[B_{2}^{*}(\delta-1)+C_{2}^{*}(2+\delta)\right], } \\
U_{p 2}= & {\left[B_{2}(\delta-1)+C_{2}(2+\delta)\right]^{2} \cdot\left[\left|C_{2}\right|^{2}\left(5+2 \delta+5 \delta^{2}\right)\right.} \\
& \left.+\left(C_{2} B_{2}^{*}+B_{2} C_{2}^{*}\right)\left(-4+5 \delta+5 \delta^{2}\right)+\left|B_{2}\right|^{2}\left(8+8 \delta+5 \delta^{2}\right)\right], \\
U_{p 3}= & \left.B_{2}^{*}(\delta-1)+C_{2}^{*}(2+\delta)\right] \cdot\left[B_{2}^{3}\left(-8+3 \delta^{2}+5 \delta^{3}\right)\right. \\
& +3 B_{2}^{2} C_{2}\left(8+2 \delta+6 \delta^{2}+5 \delta^{3}\right)+3 B_{2} C_{2}^{2}\left(-7+5 \delta+9 \delta^{2}+5 \delta^{3}\right) \\
& \left.+C_{2}^{3}\left(10+9 \delta+12 \delta^{2}+5 \delta^{3}\right)\right], \\
U_{p 4}= & {\left[B_{2}^{2}\left|B_{2}\right|^{2}\left(128+64 \delta+48 \delta^{2}+40 \delta^{3}+35 \delta^{4}\right)\right.} \\
& +B_{2}^{2}\left(3 C_{2} B_{2}^{*}+B_{2} C_{2}^{*}\right)\left(-112-8 \delta+30 \delta^{2}+55 \delta^{3}+35 \delta^{4}\right) \\
& +3 B_{2} C_{2}\left(C_{2} B_{2}^{*}+B_{2} C_{2}^{*}\right)\left(104+4 \delta+39 \delta^{2}+70 \delta^{3}+35 \delta^{4}\right) \\
& +C_{2}^{2}\left(C_{2} B_{2}^{*}+3 B_{2} C_{2}^{*}\right)\left(-94+43 \delta+75 \delta^{2}+85 \delta^{3}+35 \delta^{4}\right) \\
& \left.+C_{2}^{2}\left|C_{2}\right|^{2}\left(107+52 \delta+138 \delta^{2}+100 \delta^{3}+35 \delta^{4}\right)\right],
\end{aligned}
$$


with

$$
B_{2}=\frac{\bar{\epsilon}(r)+(1+\delta) \epsilon_{1 r}(r)}{(1+2 \delta) \epsilon_{1 r}(r)} \quad \text { and } \quad C_{2}=\frac{\bar{\epsilon}(r)-\delta \cdot \epsilon_{1 r}(r)}{(1+2 \delta) \epsilon_{1 r}(r)} .
$$

Substituting Eqs. (146)-(148) into Eq. (145), we have a differential equation for the equivalent nonlinear susceptibility $\bar{\chi}(r)$, namely,

$$
\frac{\mathrm{d} \bar{\chi}(r)}{\mathrm{d} r}=\bar{\chi}(r)\left[\frac{3 \mathrm{~d} \bar{\epsilon}(r) / \mathrm{d} r}{2 \epsilon_{2}+\bar{\epsilon}(r)}+\left(\frac{\mathrm{d} \bar{\epsilon}(r) / \mathrm{d} r}{2 \epsilon_{2}+\bar{\epsilon}(r)}\right)^{*}\right]+\bar{\chi}(r) \cdot \frac{3 y+y^{*}-3}{r}+\frac{3}{r} \cdot \frac{U}{|K|^{2} K^{2}} .
$$

From Eqs. (148) and (149), it is evident that $\chi_{i j k l}$ does not contribute to the equivalent nonlinear susceptibility, except for the cases with equal pair indices.

So far, the equivalent $\bar{\epsilon}(r)$ and $\bar{\chi}(r)$ of the anisotropic graded spherical particles with radius $r$ can be calculated, at least numerically, by solving the differential equations Eqs. (144) and (149), as long as $\epsilon_{1 r}(r), \epsilon_{1 t}(r)$ (dielectric-constant gradation profiles) and $\chi_{i j k l}$ are given. Here we would like to mention that, even though $\chi_{i j k l}$ is independent of $r$, the equivalent $\bar{\chi}(r)$ should still be dependent on $r$. This is because both $\epsilon_{1 r}(r)$ and $\epsilon_{1 t}(r)$ are $r$-dependent. Moreover, if $\chi_{i j k l}=0$, Eq. (145) admits $\bar{\chi}(r)=0$, as expected as well.

To obtain $\bar{\epsilon}(r=a)$ and $\bar{\chi}(r=a)$, we integrate Eqs. (144) and (149) numerically, for given initial conditions $\bar{\epsilon}(r \rightarrow 0)$ and $\bar{\chi}(r \rightarrow 0)$. Once $\bar{\epsilon}(r=a)$ and $\bar{\chi}(r=a)$ are calculated, we can take one step forward to work out the effective linear and nonlinear responses of the whole composite $\epsilon_{e}$ and $\chi_{e}[121]$,

$$
\epsilon_{e}=\epsilon_{2}+3 \epsilon_{2} f \frac{\bar{\epsilon}(r=a)-\epsilon_{2}}{\bar{\epsilon}(r=a)+2 \epsilon_{2}}
$$

and

$$
\chi_{e}=f \bar{\chi}(r=a)\left|\frac{3 \epsilon_{2}}{\bar{\epsilon}(r=a)+2 \epsilon_{2}}\right|^{2}\left(\frac{3 \epsilon_{2}}{\bar{\epsilon}(r=a)+2 \epsilon_{2}}\right)^{2} .
$$

\subsubsection{Exact solution for linear gradation profiles}

Based on the first-principles approach, the potentials within the graded inclusions and the host medium can be exactly obtained, when the dielectric gradation profiles are the linear radial functions with small slopes, i.e., $\epsilon_{1 r}(r)=\epsilon(0)+$ $g(r / a)$ and $\epsilon_{1 t}(r)=\epsilon(0)+h(r / a)$. Here $g[<a \epsilon(0)]$ and $h$ are two constants, and $\epsilon(0)$ denotes the linear dielectric constant at radius $r=0$.

The potentials within the graded spheres and the host medium are respectively given by

$$
\begin{aligned}
& \phi_{c}(r, \theta)=-E_{0} A_{1} \sum_{k=0}^{\infty} C_{k}\left(\frac{g r}{a \epsilon(0)}\right)^{k+1} \cos \theta, \quad r<a, \\
& \phi_{m}(r, \theta)=-E_{0} r \cos \theta+\frac{D_{1}}{r^{2}} E_{0} \cos \theta, \quad r>a,
\end{aligned}
$$

where the coefficients $A_{1}$ and $D_{1}$ have the following forms:

$$
A_{1}=\frac{3 \epsilon_{2} a}{(\epsilon(0)+g) v_{2}+2 \epsilon_{2} v_{1}} \quad \text { and } \quad D_{1}=\frac{(\epsilon(0)+g) v_{2}-\epsilon_{2} v_{1}}{(\epsilon(0)+g) v_{2}+2 \epsilon_{2} v_{1}} a^{3} .
$$

Here $v_{1}$ and $v_{2}$ are given by

$$
v_{1}=\sum_{k=0}^{\infty} C_{k}\left(\frac{g}{\epsilon(0)}\right)^{k+1} \text { and } \quad v_{2}=\sum_{k=0}^{\infty} C_{k}(k+1)\left(\frac{g}{\epsilon(0)}\right)^{k+1},
$$


with $C_{k}$ satisfying the following recurrent relation:

$$
C_{k+1}=-\frac{(k+1)(k+3)-2 h / g}{(k+2)(k+3)-2} C_{k} .
$$

The local electric field inside the anisotropic graded inclusions can be derived from the relation $\mathbf{E}=-\nabla \phi$, and we have

$$
\begin{aligned}
\mathbf{E}_{c}= & A_{1} E_{0} \sum_{k=0}^{\infty} C_{k}\left(\frac{g}{a \epsilon(0)}\right)^{k+1} r^{k}\left[(k+1) \cos \theta \mathbf{e}_{r}-\sin \theta \mathbf{e}_{\theta}\right] \\
= & A_{1} E_{0} \sum_{k=0}^{\infty} C_{k}\left(\frac{g}{a \epsilon(0)}\right)^{k+1} r^{k}\left\{k \cos \theta \sin \theta \cos \phi \mathbf{e}_{x}+k \cos \theta \sin \theta \sin \phi \mathbf{e}_{y}\right. \\
& \left.\times\left[(k+1) \cos ^{2} \theta+\sin ^{2} \theta\right] \mathbf{e}_{z}\right\} .
\end{aligned}
$$

Then, the corresponding displacement admits

$$
\begin{aligned}
\mathbf{D}_{c}= & \stackrel{\leftrightarrow}{\varepsilon}_{1}(r) \cdot \mathbf{E}_{c}=A_{1} E_{0} \sum_{k=0}^{\infty} C_{k}\left(\frac{g}{a \epsilon(0)}\right)^{k+1} r^{k}\left[\epsilon_{1 r}(r)(k+1) \cos \theta \mathbf{e}_{r}-\epsilon_{1 t}(r) \sin \theta \mathbf{e}_{\theta}\right] \\
= & A_{1} E_{0} \sum_{k=0}^{\infty} C_{k}\left(\frac{g}{a \epsilon(0)}\right)^{k+1} r^{k}\left\{\left[\epsilon_{1 r}(r)(k+1)-\epsilon_{1 t}(r)\right] \cos \theta \sin \theta \cos \phi \mathbf{e}_{x}\right. \\
& \left.+\left[\epsilon_{1 r}(k+1)-\epsilon_{1 t}(r)\right] \cos \theta \sin \theta \sin \phi \mathbf{e}_{y}+\left[\epsilon_{1 r}(r)(k+1) \cos ^{2} \theta+\epsilon_{1 t}(r) \sin ^{2} \theta\right] \mathbf{e}_{z}\right\},
\end{aligned}
$$

where $\mathbf{e}_{r}$ and $\mathbf{e}_{\theta}\left(\mathbf{e}_{x}, \mathbf{e}_{y}\right.$ and $\left.\mathbf{e}_{z}\right)$ are the unix vectors in spherical coordinates (Cartesian coordinates).

In the dilute limit, from Eq. (137), we can obtain the effective linear dielectric constant as

$$
\begin{aligned}
\epsilon_{e} & =\epsilon_{2}+\frac{1}{V E_{0}} \int_{\Omega_{i}}\left(\stackrel{\leftrightarrow}{\varepsilon}_{1}(r) \cdot \mathbf{E}-\epsilon_{2} \mathbf{E}\right) \cdot \mathbf{e}_{z} \mathrm{~d} V \\
& =\epsilon_{2}+3 f \epsilon_{2} \frac{\left[\epsilon(0)-\epsilon_{2}\right] v_{1}+g v_{3}+2 h v_{4}}{[\epsilon(0)+g] v_{2}+2 \epsilon_{2} v_{1}}
\end{aligned}
$$

where

$$
v_{3}=\sum_{k=0}^{\infty} C_{k} \frac{1+k}{4+k}\left(\frac{g}{\epsilon(0)}\right)^{k+1} \quad \text { and } \quad v_{4}=\sum_{k=0}^{\infty} C_{k} \frac{1}{4+k}\left(\frac{g}{\epsilon(0)}\right)^{k+1} .
$$

On the other hand, the substitution of Eq. (153) into Eq. (138) yields

$$
\begin{aligned}
\chi_{e}= & \frac{1}{V} \sum_{i j k l} \int_{\Omega_{i}} \chi_{i j k l} E_{i} E_{j} E_{k} E_{l}^{*} \mathrm{~d} V \\
= & f\left[\left(\chi_{x x y y}+\chi_{y x x y}+\chi_{x y x y}+\chi_{x y y x}+\chi_{y x y x}+\chi_{y y x x}+3 \chi_{x x x x}+3 \chi_{y y y y}\right) U_{q 1}\right. \\
& \times\left(\chi_{x x z z}+\chi_{x z x z}+\chi_{z x x z}+\chi_{y y z z}+\chi_{y z y z}+\chi_{z y y z}\right) U_{q 2}+\left(\chi_{z z x x}+\chi_{z x z x}\right. \\
& \left.\left.+\chi_{x z z x}+\chi_{z z y y}+\chi_{y z z y}+\chi_{z y z y}\right) U_{q 3}+\chi_{z z z z} U_{q 4}\right]
\end{aligned}
$$


where

$$
\begin{aligned}
& U_{q 1}=\frac{1}{105}\left|A_{2}\right|^{2} A_{2}^{2} \sum_{k_{1}=0}^{\infty} \sum_{k_{2}=0}^{\infty} \sum_{k_{3}=0}^{\infty} \sum_{k_{4}=0}^{\infty}\left\{\left[C_{k_{1}} C_{k_{2}} C_{k_{3}} C_{k_{4}}\left(\frac{g}{\epsilon(0)}\right)^{k_{1}+k_{2}+k_{3}+3}\right]\right. \\
& \left.\times\left[\left(\frac{g}{\epsilon(0)}\right)^{k_{4}+1}\right]^{*} \frac{k_{1} k_{2} k_{3} k_{4}}{3+k_{1}+k_{2}+k_{3}+k_{4}}\right\}, \\
& U_{q 2}=3\left|A_{2}\right|^{2} A_{2}^{2} \sum_{k_{1}=0}^{\infty} \sum_{k_{2}=0}^{\infty} \sum_{k_{3}=0}^{\infty} \sum_{k_{4}=0}^{\infty}\left\{\left[C_{k_{1}} C_{k_{2}} C_{k_{3}} C_{k_{4}}\left(\frac{g}{\epsilon(0)}\right)^{k_{1}+k_{2}+k_{3}+3}\right]\right. \\
& \left.\times\left[\left(\frac{g}{\epsilon(0)}\right)^{k_{4}+1}\right]^{*} \frac{k_{1} k_{2}}{3+k_{1}+k_{2}+k_{3}+k_{4}}\left[\frac{1}{15}+\frac{1}{35}\left(k_{3}+k_{4}\right)+\frac{1}{63} k_{3} k_{4}\right]\right\}, \\
& U_{q 3}=3\left|A_{2}\right|^{2} A_{2}^{2} \sum_{k_{1}=0}^{\infty} \sum_{k_{2}=0}^{\infty} \sum_{k_{3}=0}^{\infty} \sum_{k_{4}=0}^{\infty}\left\{\left[C_{k_{1}} C_{k_{2}} C_{k_{3}} C_{k_{4}}\left(\frac{g}{\epsilon(0)}\right)^{k_{1}+k_{2}+k_{3}+3}\right]\right. \\
& \left.\times\left[\left(\frac{g}{\epsilon(0)}\right)^{k_{4}+1}\right]^{*} \frac{k_{3} k_{4}}{3+k_{1}+k_{2}+k_{3}+k_{4}}\left[\frac{1}{15}+\frac{1}{35}\left(k_{1}+k_{2}\right)+\frac{1}{63} k_{1} k_{2}\right]\right\}, \\
& U_{q 4}=3\left|A_{2}\right|^{2} A_{2}^{2} \sum_{k_{1}=0}^{\infty} \sum_{k_{2}=0}^{\infty} \sum_{k_{3}=0}^{\infty} \sum_{k_{4}=0}^{\infty}\left\{\left[C_{k_{1}} C_{k_{2}} C_{k_{3}} C_{k_{4}}\left(\frac{g}{\epsilon(0)}\right)^{k_{1}+k_{2}+k_{3}+3}\right]\right. \\
& \times\left[\left(\frac{g}{\epsilon(0)}\right)^{k_{4}+1}\right]^{*} \frac{1}{3+k_{1}+k_{2}+k_{3}+k_{4}}\left[1+\frac{1}{3} \sum_{i=1}^{4} k_{i}+\frac{1}{5} \sum_{i=1}^{3} \sum_{j=i+1}^{4} k_{i} k_{j}\right. \\
& \left.\left.\times \frac{1}{7} \sum_{i=1}^{2} \sum_{j=i+1}^{3} \sum_{l=j+1}^{4} k_{i} k_{j} k_{l}+\frac{1}{9} k_{1} k_{2} k_{3} k_{4}\right]\right\},
\end{aligned}
$$

with $A_{2}=\left(3 \epsilon_{2}\right) /\left\{[\epsilon(0)+g] v_{2}+2 \epsilon_{2} v_{1}\right\}$.

To illustrate the NADEDA, we first perform numerical calculations for the linear dielectric gradation profiles, that is, $\epsilon_{1 r}(r)=\epsilon(0)+g r / a$ (radial dielectric constant), and $\epsilon_{1 t}(r)=\epsilon(0)+h r / a$ (tangential dielectric constant). In this situation, the exact results for $\epsilon_{e}$ and $\chi_{e}$ exist, and thus it allows us to show the correctness of the NADEDA. For model calculations, we set $h>g$ (Note that our formulae can still be used for $h \leqslant g$ ). For the NADEDA, we numerically integrate Eqs. (144) and (149) by using Mathematica with the initial radius $r=0.001$.

In Fig. 38, the effective linear dielectric constant $\left(\epsilon_{e}\right)$ is plotted as a function of the dielectric constant of anisotropic graded particles at radius $r=0[\epsilon(0)]$, for various gradients $h$ and $g$. It is shown that $\epsilon_{e}$ increases monotonically with the increase of $\epsilon(0)$. Moreover, increasing the gradient $g$ causes $\epsilon_{e}$ to increase as well. This can be understood by the fact that the increases of both $\epsilon(0)$ and $g$ lead to the increase of the equivalent dielectric constant $\bar{\epsilon}(a)$ of the graded particles, thus increasing the effective response of the whole system. For $\epsilon_{e}$, the NADEDA shows good agreement with the first-principles approach.

Next, we investigate the effective third-order nonlinear susceptibility. Let us set the tensorial dielectric susceptibility of the particles to be independent of $r$, in an attempt to focus on the nonlinearity enhancement. As a result, it is shown that the nonlinearity enhancement decreases with the increase of $\epsilon(0)$ and $g$. As mentioned above, for larger $\epsilon_{e}$ and $g$, the graded inclusions possess a larger equivalent dielectric constant, and hence the $i$ th Cartesian component of the local field should become more weak accordingly. Then, the weaker effective nonlinear susceptibility is obtained. As displayed in Fig. 39, we show three typical cases of nonlinearity enhancement. Here, all the physical parameters in use are real, and thus the nonlinearity enhancement for $\chi_{z z x x}$ (the only nonzero component) is the same as that for $\chi_{x x z z}$. 


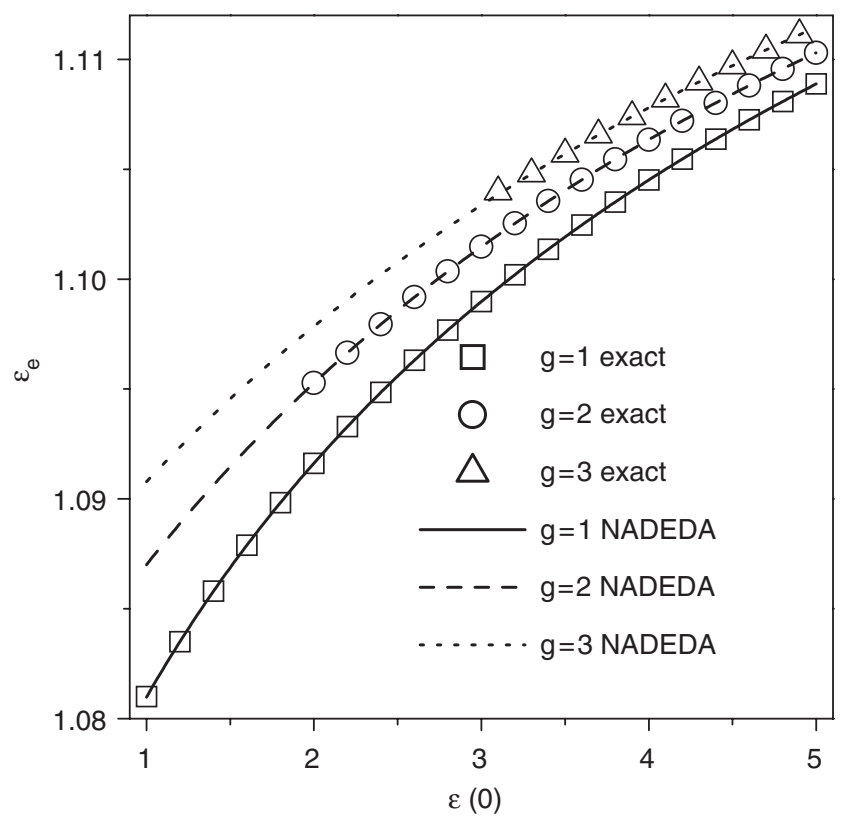

Fig. 38. The effective linear dielectric constant $\left(\epsilon_{e}\right)$ versus the dielectric constant of the spherical core with radius $r=0^{+}[\epsilon(0)]$, for the linear dielectric gradation profiles with various radial gradients $g$ [60]. Parameters: volume fraction $f=0.05$, the tangential gradient $h=8$. Lines: numerical results obtained from the NADEDA [Eqs. (144) and (150)]; Symbols: exact results predicted by the first-principles approach [Eq. (155)]. Note that the exact results are available for $\epsilon(0)>g$.

Moreover, for other nonzero components of the tensorial nonlinear susceptibility, the nonlinearity enhancement will be the same as one of these shown in Fig. 39. For example, Fig. 39(a) can also show the nonlinearity enhancement for $3 \chi_{x x x x}, 3 \chi_{y y y y}, \chi_{y x x y}$, etc. Again, the excellent agreement is numerically demonstrated between the first-principles approach and the NADEDA [Eqs. (144), (149) and (151)].

In what follows, we shall investigate the surface plasma resonance effect on the nonlinear metal-dielectric composite. As a model calculation, we assume the radial and tangential dielectric constants for the graded metal particles to be Drude-like, namely,

$$
\epsilon_{1 r}(r)=1-\frac{\omega_{p r}^{2}(r)}{\omega(\omega+\mathrm{i} \gamma)} \quad \text { and } \quad \epsilon_{1 t}(r)=1-\frac{\omega_{p t}^{2}(r)}{\omega(\omega+\mathrm{i} \gamma)},
$$

where $\omega_{p r}(r)$ and $\omega_{p t}(r)$ are the radius-dependent radial and tangential plasma frequencies, respectively, and $\gamma$ is the damping coefficient. For the linear dielectric host, we choose $\epsilon_{2}=1.77$ (a typical dielectric constant of water). We further assume $\omega_{p r}(r)$ and $\omega_{p t}(r)$ to be

$$
\omega_{p r}(r)=\omega_{p}\left(1-k_{r} \cdot \frac{r}{a}\right) \quad \text { and } \quad \omega_{p t}(r)=\omega_{p}\left(1-k_{t} \cdot \frac{r}{a}\right), \quad r<a .
$$

The above form is quite physical for $0<k_{r}\left(k_{t}\right)<1$, since the center of grains can be better metallic so that $\omega_{p}(r)$ is larger, while the boundary of the grain may be poorer metallic so that $\omega_{p}(r)$ is much smaller. In fact, such a variation can also appear owing to the temperature effect [114]. Moreover, we choose $k_{t} \leqslant k_{r}$, in view of the strong metallic behavior in the tangential direction.

Fig. 40 displays the real and imaginary parts of effective dielectric constant $\epsilon_{e}$ as a function of the incident angular frequency $\omega / \omega_{p}$. For $k_{r}=0$, there exists a frequency region, where the real part of the effective dielectric constant is negative. With increasing $k_{r}$, this region becomes narrow generally, in accompanied with less negative $\operatorname{Re}\left(\epsilon_{e}\right)$ [see Fig. 40(a)]. This is due to the fact that increasing $k_{r}$ decreases the influence of the metallic behavior [owing to the decrease of $\left.\omega_{p r}(r)\right]$. In the mean time, the sharp peak for $\operatorname{Im}\left(\epsilon_{e}\right)$ turn weak with $k_{r}$ [see Fig. 40(b)]. Furthermore, for $k_{r} \neq 0$, the continuous resonant bands in the high frequency region appear always, and this region becomes more 

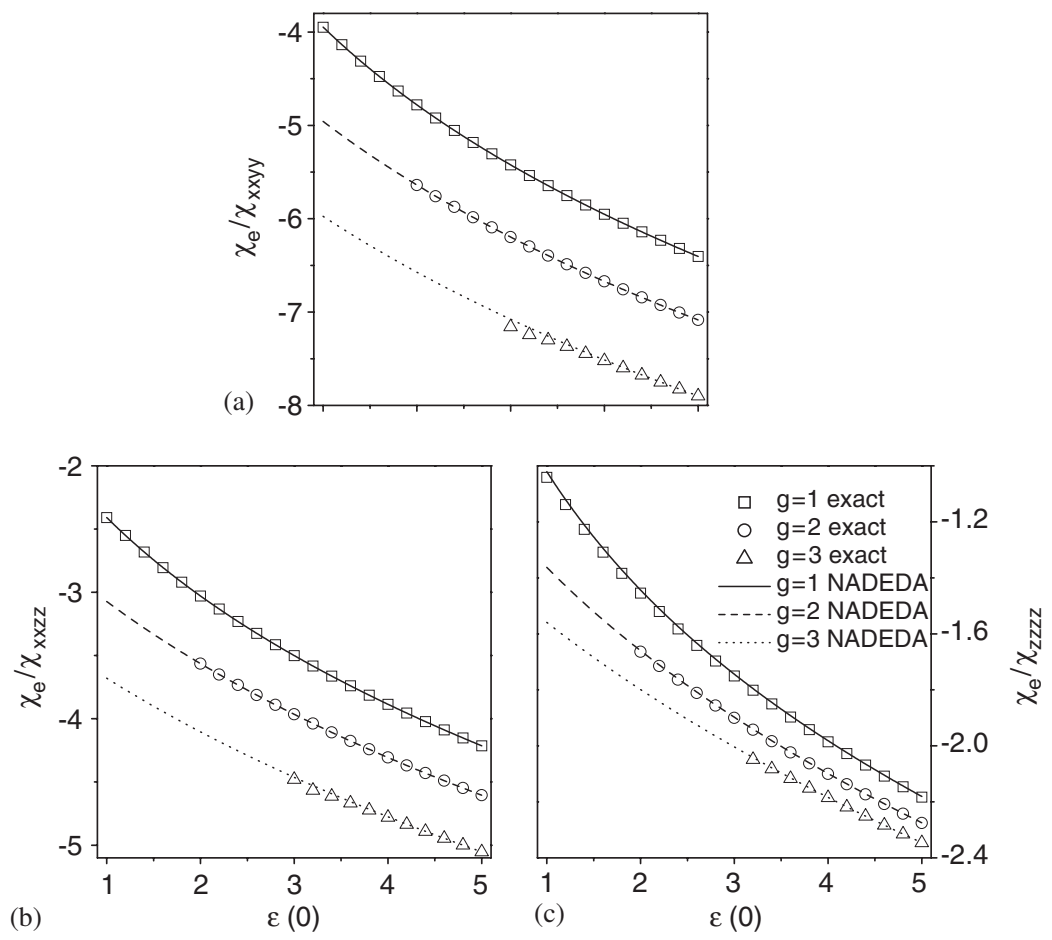

Fig. 39. (a) $\chi_{e} / \chi_{x x y y}$ versus $\epsilon(0)$, for the linear dielectric gradation profiles with various $g$, at $h=8$ [60]. Here $\chi_{x x y y}$ is the only nonzero component of the tensorial nonlinear susceptibility. Lines: numerical results obtained from the NADEDA [Eqs. (144), (149) and (151)]; Symbols: exact results predicted by the first-principles approach [Eq. (156)]. (b) Same as (a), but $\chi_{e} / \chi_{x x z z}$ versus $\epsilon(0)$, with $\chi_{x x z z}$ being the only nonzero component. (c) Same as (a), but $\chi_{e} / \chi_{z z z z}$ versus $\epsilon(0)$, with $\chi_{z z z z}$ being the only nonzero component.

broad as $k_{r}$ increases. In this case, the appearance of the resonant bands results from the radius-dependent plasma frequency $\omega_{p}(r)$. This phenomenon has already been observed, when a shell model [78] or nonspherical model [124] was taken into account. In our previous works [78,124], a broad continuous spectrum is shown to be around the larger pole in the corresponding spectral density function. Here, the graded particles under consideration can be regarded as a construction of multi shells, which hence should be expected to yield the broader spectra for the optical absorption $\left[\operatorname{Im}\left(\epsilon_{e}\right)\right]$. In addition, we note that, as $k_{r}$ increases, both the surface plasma frequency and the center of resonant bands are red-shifted. In particular, for larger $k_{r}$, the resonant bands can become broader, owing to strong inhomogeneity inside the particles.

Then, we speculate on how gradation and anisotropy affect the optical nonlinearity enhancement in metal-dielectric composites. As shown in Fig. 41, no matter which component of the nonlinear susceptibility tensor is nonzero, $\chi_{e}$ can be substantially enhanced within a certain frequency region. In particular, this enhancement becomes quite strong for $\chi_{z z z z}$ (the only nonzero component). In fact, the physical origin of this huge enhancement is the large increase in the local field component $E_{z}$. In addition, the nonlinearity enhancement will become more strong, for the system with a larger $k_{r}$ which is related to a higher contrast between $\epsilon_{1 t}$ and $\epsilon_{1 r}$. For example, $\left|\chi_{e} / \chi_{z z z z}\right|>10^{4}$ in the frequency region $0.2 \leqslant \omega / \omega_{p} \leqslant 1.0$ for $k_{r}=1$. From Fig. 41, we also find that the optical nonlinearity enhancement obtained for four nonzero components, respectively, displays the similar qualitative behaviors. This should be in contrast to those observed in a polycrystalline quasi-one-dimensional conductor [121,123,125], where the effective optical nonlinearity for four elements of the nonlinear susceptibility tensor exhibit quite different behaviors [121] (the differences become more distinct by using spectral representation approximation [123]). Actually, the differences result from two different kinds of dielectric anisotropy (and hence two different kinds of tensorial dielectric constants) under consideration. We focus on the particles with spatially varying, but spherically symmetric, dielectric anisotropy, whereas, in the previous works $[121,123,125]$, the authors studied uniaxial anisotropy in the Cartesian coordinate system. 

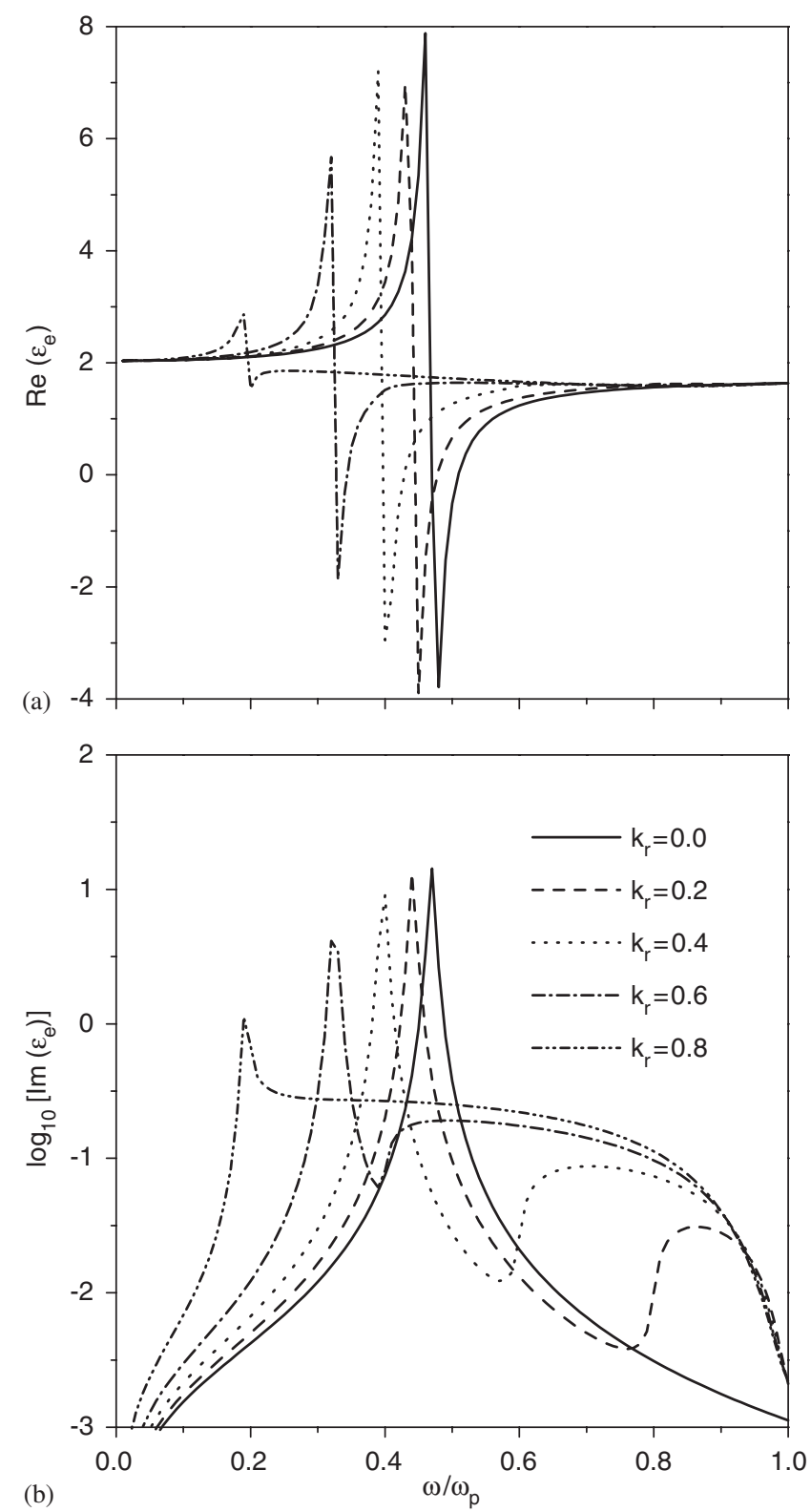

Fig. 40. The real and imaginary parts of $\epsilon_{e}$ versus frequency $\omega / \omega_{p}$, for various $k_{r}$ [60]. Parameters: $\omega_{p} / \gamma=0.01$ and $k_{t}=0$.

Although the optical nonlinearity enhancements for four typical nonzero components of the nonlinear susceptibility $\left(\chi_{i j k l}\right)$ take on quite similar behaviors, their contributions to the magnitude of the effective optical nonlinearity are different (see Fig. 42). As shown in Fig. 42, the strongest (weakest) nonlinearity enhancement occurs for the case with $\chi_{z z z z}\left(\chi_{x x y y}\right)$ being only nonzero component. Moreover, the differences between the two cases of $\chi_{x x z z} \neq 0$ only and $\chi_{z z x x} \neq 0$ only are clearly shown for $\operatorname{Re}\left(\chi_{e} / \chi_{1}\right)$ and $\operatorname{Im}\left(\chi_{e} / \chi_{1}\right)$.

For practical applications, a most useful parameter is the figure of merit (FOM), which is defined as the ratio of $\left|\chi_{e}\right|$ to $\operatorname{Im}\left(\epsilon_{e}\right)$. In Fig. 43, we investigate the figure of merit. Here the only nonzero component is assumed to be $\chi_{z z z z}$. We find that the increase of $k_{r}$ (namely, the rapid decrease of the radial metallic behavior) results in a large enhancement of the FOM, especially in the high frequency range [see Fig. 43(a)]. However, the increase of $k_{t}$ (i.e., the rapid decrease of the tangential metallic behavior) causes the FOM in the high-frequency region to decrease [see Fig. 43(b)]. 


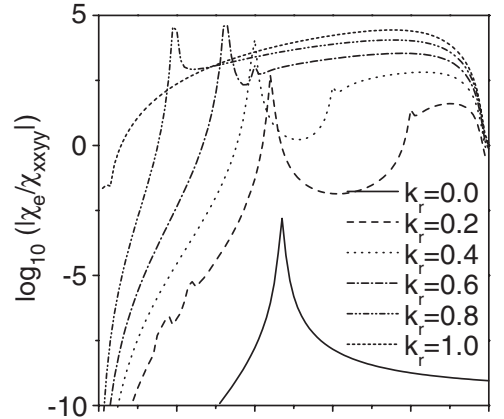

(a)

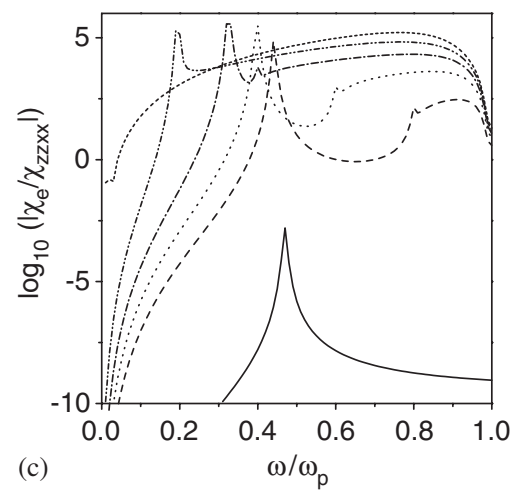

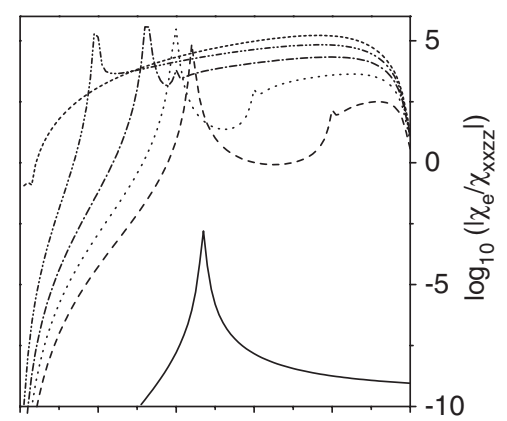

(b)

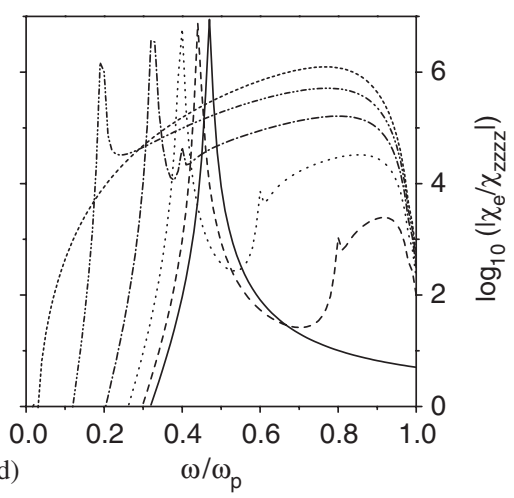

Fig. 41. Same as Fig. 40, but (a) $\left|\chi_{e} / \chi_{x x y y}\right|$ versus $\omega / \omega_{p}$, with $\chi_{x x y y}$ being the only nonzero component; (b) $\left|\chi_{e} / \chi_{x x z z}\right|$ versus $\omega / \omega_{p}$, with $\chi_{x x z z}$ being the only nonzero component; (c) $\left|\chi_{e} / \chi_{z z x x}\right|$ versus $\omega / \omega_{p}$, with $\chi_{z z x x}$ being the only nonzero component; (d) $\left|\chi_{e} / \chi_{z z z z}\right|$ versus $\omega / \omega_{p}$, with $\chi_{z z z z}$ being the only nonzero component $[60]$.

For instance, we attain FOM $>10^{5}$ (which is quite large) in the frequency region $0.3 \leqslant \omega / \omega_{p} \leqslant 1.0$ for $k_{r}=0.6$ and $k_{t}=0$. Therefore, it is possible to achieve a large figure of merit by introducing the radial gradation and keeping the tangential dielectric constant unchanged.

We have developed an NADEDA to investigate the effective linear dielectric constant and third-order nonlinear susceptibility of composite media consisting of nonlinear inclusions with spatially varying dielectric anisotropy. Alternatively, based on the first-principles approach, we have derived the exact expressions for $\epsilon_{e}$ and $\chi_{e}$, for the linear dielectric-constant profiles with small slopes. To our interest, excellent agreement is found between the approximation results (NADEDA) and the exact results (first-principles approach). It is worth noting that exact solutions are very few in composite research, and thus our NADEDA provides an effective way to estimate the effective nonlinear properties in composite media consisting of anisotropic graded inclusions.

An application, we apply the NADEDA to study the surface plasma resonance effect on the effective linear dielectric constant, the optical nonlinearity enhancement and the figure of merit in metal-dielectric composites, in which the metal particles possess the tensorial dielectric constants with dielectric gradation profiles. It is found that the gradation profiles in radial dielectric constants are a useful way to control the local-field effects, thus being able to enhance the figure of merit hugely.

The present methods are strictly valid in the dilute limit. The presence of both gradation and dielectric anisotropy is shown to be helpful to achieve the large figure of merit, but unable to realize the separation of the absorption peak from the nonlinearity enhancement peak. In this regard, we may intentionally manipulate composite microstructures, e.g., by using the shape distribution of graded inclusions [7], and by using fractal [6] and anisotropic microstructures [90] with large volume fractions. When the volume fraction of graded inclusions is large, percolation behaviors can occur. To this end, the further broadening of the enhancement peak as well as the desired separation of the optical absorption from the nonlinearity peak is expected to be realized. 

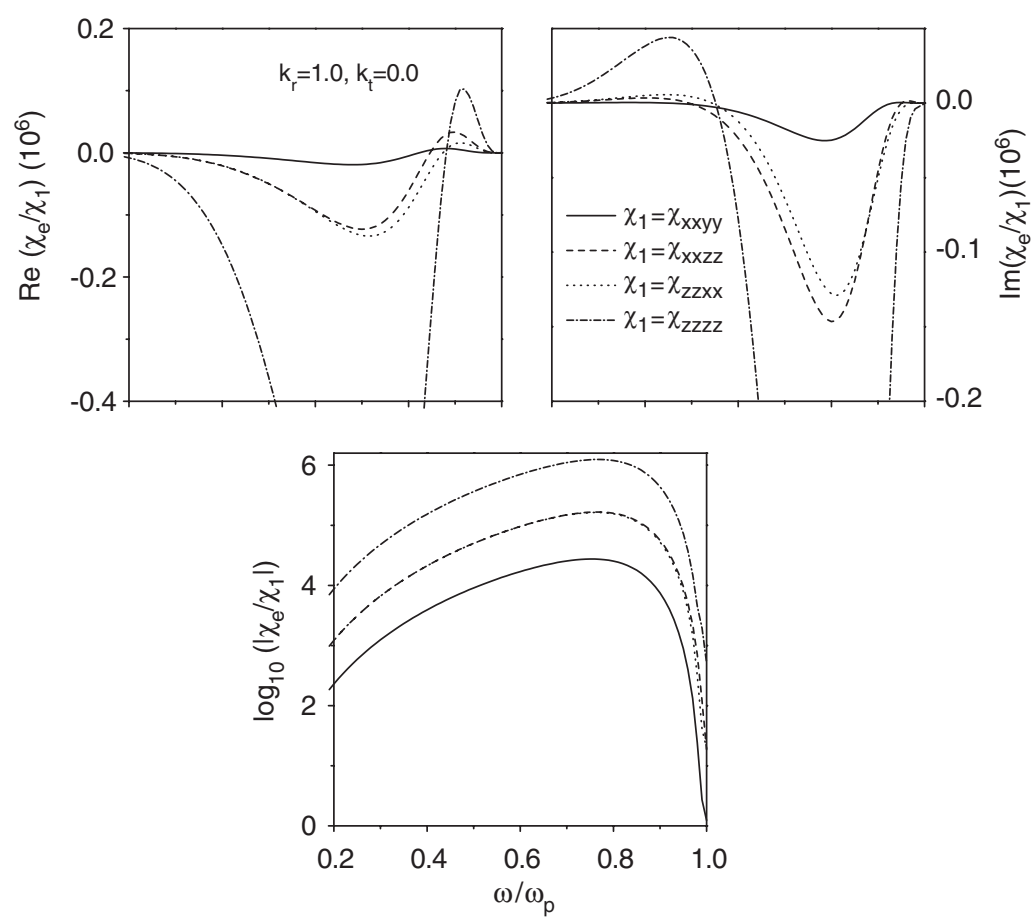

Fig. 42. The real and imaginary parts, and the modulus of the optical nonlinearity enhancement for $k_{r}=1$ and $k_{t}=0$ [60].

To sum up, we put forth an NADEDA, in an attempt to discuss the effects of gradation as well as anisotropy on the optical properties of composite media. For the linear dielectric-constant profiles, the NADEDA has been numerically demonstrated in good agreement with the first-principles approach. Interestingly, both the huge nonlinearity enhancement and the large figure of merit are shown to be achievable by the presence of gradation as well as local anisotropy inside the inclusions.

\subsection{Spectral representation for understanding the effective dielectric constant of graded and multilayer composites}

The idea of using a spectral representation for understanding the effective dielectric constant of graded and multilayer composites has been motivated by a recent study of the optical absorption spectrum of a graded metallic film [21]. In that work, a broad surface plasmon absorption band was observed in addition to a strong Drude absorption peak at zero frequency. Such a broad absorption band has been shown to be responsible for the enhanced nonlinear optical response as well as an attractive FOM. Yuen et al. [90] pointed out that such an absorption spectrum, being related to the imaginary part of the effective dielectric constant, should equally well be reflected in the Bergman-Milton spectral representation of the effective dielectric constant [3,126,127].

Bergman-Milton spectral representation was originally developed for calculating the effective dielectric constant and other response functions of two-component composites [3,126,127]. However, the two concerned components are all homogeneous. Therefore, it is worth extending the spectral representation to graded composite materials. The work on graded films is just a simple example of a more general graded composite in three dimensions. One of the main purpose of this idea help to identify the physical origin of the broad absorption band. It turns out that, unlike in the case of homogeneous materials, the characteristic function of a graded composite is a continuous function because of the continuous variation of the dielectric function within the constituent component.

We shall apply our theory to a special case of graded composites, i.e., multilayer material, which is more convenient to fabricate in practice than graded material [128], and many algorithms are now available for designing of multilayer coatings $[129,130]$. Thus, the present idea is necessary in the sense that we shall discuss the multilayer effect as the number of layers inside the material increases. In this regard, this idea should be expected to have practical relevance. 

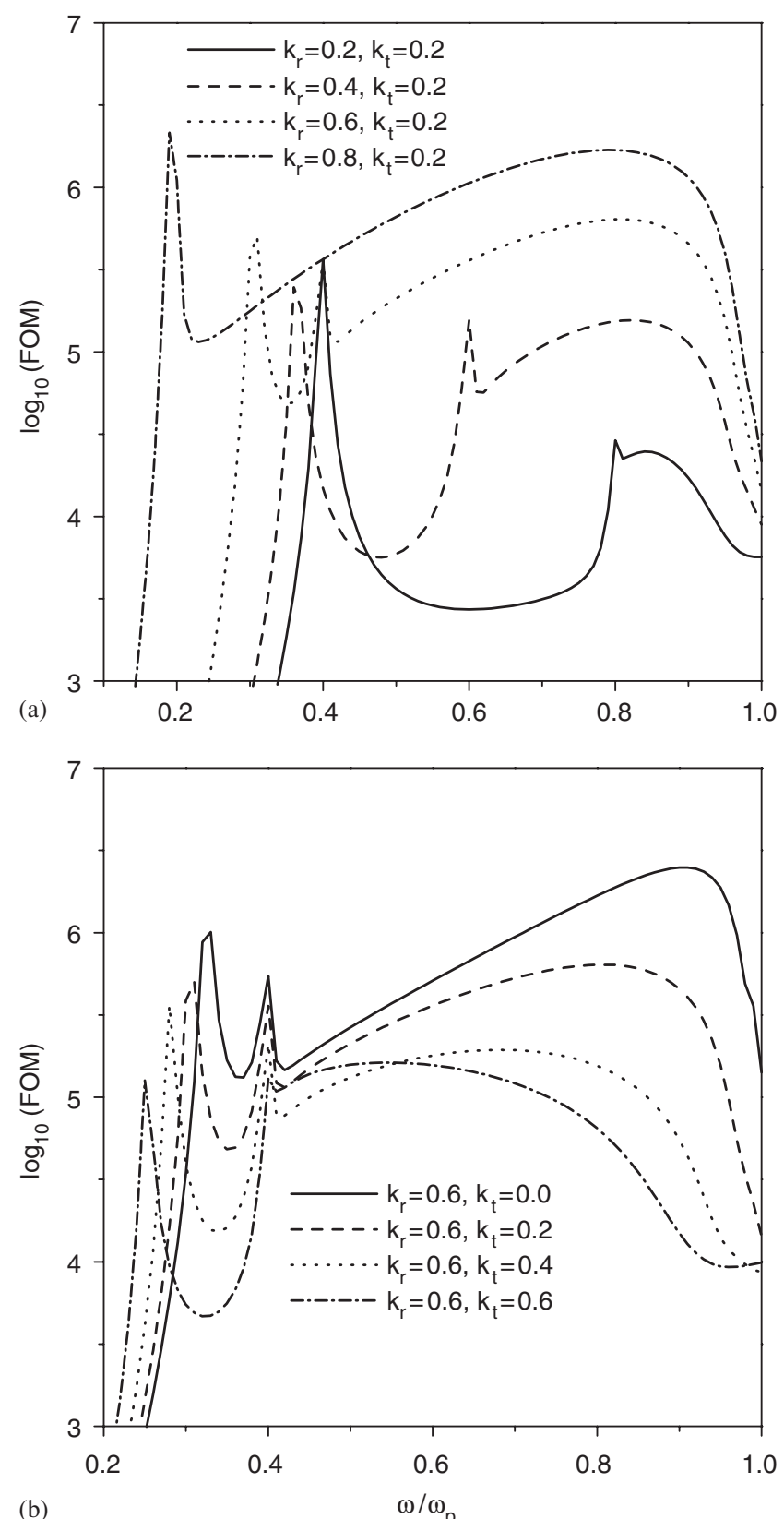

Fig. 43. The figure of merit $\equiv\left|\chi_{e} / \chi_{z z z z}\right| / \operatorname{Im}\left(\epsilon_{e}\right)$ versus $\omega / \omega_{p}$, with $\chi_{z z z z}$ being the only nonzero component [60].

As the number of layers $N$ increases, we shall show a gradual transition from sharp peaks to a broad continuous band until the graded composite results are recovered by the limit of $N \rightarrow \infty$.

We consider a two-component composite in which graded inclusions of dielectric constant $\epsilon_{1}(\mathbf{r})$ are embedded in a homogeneous host medium of dielectric constant $\epsilon_{2}$. It is noted that the dielectric constant $\epsilon_{1}(\mathbf{r})$ is a gradation profile as a function of the position $\mathbf{r}$. And we will restrict our discussion and calculation to the quasi-static approximation, i.e., $d c / \omega \leqslant 1$, where $d$ is the characteristic size of the inclusion, $c$ is the speed of light in vacuum and $\omega$ is the frequency of the applied field. In the quasi-static approximation, the whole graded structure can be regarded as an effective 
homogeneous one with effective (overall) linear dielectric constant defined as [74]

$$
\epsilon_{e}=\frac{1}{V} \int \frac{\mathbf{E} \cdot \mathbf{D}}{E_{0}^{2}} \mathrm{~d} V,
$$

where $E_{0}$ is the applied electric field along $z$ direction, $\mathbf{E}$ and $\mathbf{D}$ are the local electric field and local displacement, respectively.

The object of the present section is to solve the Laplace's equation

$$
\nabla \cdot(\epsilon(\mathbf{r}) \nabla \phi(\mathbf{r}))=0
$$

subject to the boundary condition $\phi_{0}=-E_{0} z$. The dielectric function $\epsilon(\mathbf{r})$ varies from component to component but has a fixed mathematical expression for a given component. It can be expressed as [3]

$$
\epsilon(\mathbf{r})=\epsilon_{2}\left[1-\frac{1}{s} \eta(\mathbf{r})\right]
$$

where $s=\left[1-\epsilon_{\mathrm{ref}} / \epsilon_{2}\right]^{-1}$ is the material parameter and $\epsilon_{\mathrm{ref}}$ is some reference dielectric constant in the graded component. We are considering a microstructure gradient $\eta(r)$ in Eq. (161). Thus, the following spectral representation is valid for arbitrary gradation (microstructure) profiles. The characteristic function $\eta(\mathbf{r})$ may be written in terms of a real function $f(\mathbf{r})$ as

$$
\eta(\mathbf{r})= \begin{cases}1+f(\mathbf{r}) & \text { in inclusion, } \\ 0 & \text { in host }\end{cases}
$$

which accords for the microstructure of graded composites. The function $f(\mathbf{r})$ depends on the specific variation of the dielectric constant in the inclusion component. For homogeneous constituent component, i.e., $f(\mathbf{r})=0, \eta(\mathbf{r})=1$ in the inclusion component, while $\eta(\mathbf{r})=0$ in the host medium. For graded systems, $\eta(\mathbf{r})$ can be a continuous function in the inclusion component because of the continuous variation of the dielectric function within the inclusion component. Thus, Eq. (159) can be solved

$$
\phi(\mathbf{r})=-E_{0} z+\frac{1}{s} \int \mathrm{d} V^{\prime} \eta\left(\mathbf{r}^{\prime}\right) \nabla^{\prime} G\left(\mathbf{r}-\mathbf{r}^{\prime}\right) \cdot \nabla^{\prime} \phi\left(\mathbf{r}^{\prime}\right)
$$

where $G\left(\mathbf{r}-\mathbf{r}^{\prime}\right)$ is a Green's function satisfying:

$$
\begin{aligned}
& \nabla^{2} G\left(\mathbf{r}-\mathbf{r}^{\prime}\right)=-\delta^{3}\left(\mathbf{r}-\mathbf{r}^{\prime}\right) \text { for } \mathbf{r} \text { in } \mathrm{V}, \\
& G=0 \text { for } \mathbf{r} \text { on the boundary. }
\end{aligned}
$$

In order to obtain a solution for Eq. (160), we introduce an integral-differential Hermitian operator $\hat{\Gamma}$, which satisfies

$$
\hat{\Gamma} \equiv \int \mathrm{d} V^{\prime} \eta\left(\mathbf{r}^{\prime}\right) \nabla^{\prime} G\left(\mathbf{r}-\mathbf{r}^{\prime}\right) \cdot \nabla^{\prime}
$$

and define an inner product as

$$
\langle\phi \mid \psi\rangle=\int \mathrm{d} V \eta(\mathbf{r}) \nabla \phi^{*} \cdot \nabla \psi
$$

With the above definitions, Eq. (162) can be simplified to

$$
\phi(\mathbf{r})=-E_{0} z+\frac{1}{s} \hat{\Gamma} \phi(\mathbf{r}) .
$$

Let $s_{n}$ and $\left|\phi_{n}\right\rangle$ be the $n$th eigenvalue and eigenfunction of operator $\hat{\Gamma}$. Then, the generalized eigenvalue problem becomes

$$
\nabla \cdot\left(\eta(\mathbf{r}) \nabla \phi_{n}\right)=s_{n} \nabla^{2} \phi_{n} .
$$


The potential $|\phi\rangle$ can be expanded in series of eigenfunctions,

$$
|\phi\rangle \equiv \sum_{n}\left(\frac{s}{s_{n}-s}\right) \frac{\left|\phi_{n}\right\rangle\left\langle\phi_{n} \mid z\right\rangle}{\left\langle\phi_{n} \mid \phi_{n}\right\rangle},
$$

where we choose $E_{0}=1$ for convenience. Since $\eta(\mathbf{r})$ is a real function, the eigenvalues $s_{n}$ will be real. Also, for graded component, $\eta(\mathbf{r})$ is a continuous function, which will cover the full region, i.e., $-\infty \leqslant \eta(\mathbf{r}) \leqslant \infty$. Therefore, the eigenvalues $s_{n}$, which depend on the continuously graded microstructure $\eta(\mathbf{r})$, do not lie within the interval $[0,1]$ but extend to $-\infty \leqslant s_{n} \leqslant \infty$ as first pointed by Gu and Gong [33] for three-component composites case. However, eigenvalues $s_{n}$ still lie in $[0,1]$ for $0 \leqslant \eta(\mathbf{r}) \leqslant 1$.

We are now in the position to find an analytical representation for the effective dielectric constant $\epsilon_{e}$ according to Eq. (159). We take advantage of Green's theorem, the boundary condition $\phi_{0}=-z$, and the Maxwell equation $\nabla \cdot \mathbf{D}=0$ to obtain the effective dielectric constant

$$
\begin{aligned}
\frac{\epsilon_{e}}{\epsilon_{2}} & =\frac{1}{\epsilon_{2} V} \int(-\nabla \phi) \cdot \mathbf{D} \mathrm{d} V \\
& =\frac{-1}{V} \int \hat{\mathbf{z}} \cdot\left[\left(1-\frac{1}{s} \eta(\mathbf{r})\right) \nabla \phi\right] \mathrm{d} V \\
& =1+\frac{1}{s V}\langle z \mid \phi\rangle .
\end{aligned}
$$

If we now introduce the reduced response [3]

$$
F(s)=1-\frac{\epsilon_{e}}{\epsilon_{2}},
$$

and substitute Eq. (164) into Eq. (165) we find

$$
F(s)=\frac{1}{V} \sum_{n} \frac{\left|\left\langle z \mid \phi_{n}\right\rangle\right|^{2}}{\left\langle\phi_{n} \mid \phi_{n}\right\rangle}\left(\frac{1}{s-s_{n}}\right) .
$$

We can now express the effective dielectric constant as

$$
\epsilon_{e}=\epsilon_{2}\left(1-\sum_{n} \frac{f_{n}}{s-s_{n}}\right),
$$

where $f_{n}$ is given by

$$
f_{n}=\frac{1}{V} \frac{\left|\left\langle z \mid \phi_{n}\right\rangle\right|^{2}}{\left\langle\phi_{n} \mid \phi_{n}\right\rangle} .
$$

Using the above equations, we obtain the following sum rule:

$$
\begin{aligned}
\sum_{n} f_{n} & =\frac{1}{V}\langle z \mid z\rangle \\
& =\frac{1}{V} \int \mathrm{d} V \eta(\mathbf{r}) \nabla z \cdot \nabla z \\
& =\frac{1}{V} \int \mathrm{d} V \eta(\mathbf{r}) .
\end{aligned}
$$

It is worth noting that the sum rule will not equal to the volume fraction of inclusion. This is different from the Bergman-Milton spectral representation for two homogeneous systems, in which the sum rule equals to the volume fraction of the inclusion. 
When the operator $\hat{\Gamma}$ has a continuous spectrum, Eq. (167) should be replaced with the integral form

$$
\epsilon_{e}=\epsilon_{2}\left(1-\int \mathrm{d} s^{\prime} \frac{m\left(s^{\prime}\right)}{s-s^{\prime}}\right)
$$

where $m\left(s^{\prime}\right)$ is the spectral density function. Then, the reduced response becomes

$$
F(s)=\int \mathrm{d} s^{\prime} \frac{m\left(s^{\prime}\right)}{s-s^{\prime}} .
$$

If we write $s$ as $s+i 0^{+}$, the right side of Eq. (170) becomes

$$
P \int \mathrm{d} s^{\prime} \frac{m\left(s^{\prime}\right)}{s-s^{\prime}}-\mathrm{i} \pi m(s),
$$

and thus, $m\left(s^{\prime}\right)$ is given through the limiting process

$$
m\left(s^{\prime}\right)=-\frac{1}{\pi} \operatorname{Im}\left[F\left(s^{\prime}+\mathrm{i} 0^{+}\right)\right] .
$$

This final result is identical in form to Bergman's expression for the analogous function in scalar composite materials. However, there are differences in the derivation, namely, the definition of the inner product Eq. (163), the continuous graded microstructure $\eta(\mathbf{r})$, the sum rule, as well as the range of eigenvalues $s_{n}$.

From Eq. (169) it is evident that if the spectral density function $m\left(s^{\prime}\right)$ is known, the effective dielectric constant can be obtained accurately, and vice versa. The spectral representation has been used to analyze the effective dielectric properties of composites. Recently, Levy and Bergman [131] also used it in their study of nonlinear optical susceptibility. In this regard, Sheng and coworkers [132] developed a practical algorithm for calculating the effective dielectric constants based on the spectral representation. In what follows, we restrict ourselves to a graded composite both in one dimension and three dimensions, and corresponding multilayer composites.

\subsubsection{Spectral density function of a graded film}

We consider a graded dielectric film of width $L$, in which two media meet at a planar interface as shown in Fig. 44 (a). The first medium $\epsilon_{1}(z)$ varies along $z$-axis, while the second medium $\epsilon_{2}$ is homogeneous. We define the graded microstructure as

$$
\eta(z)= \begin{cases}1+a z, & 0<z \leqslant h \\ 0, & h<z<L,\end{cases}
$$

where $a$ and $h$ are real constants. They can be varied to describe different graded films. Thus, according to Eq. (161), the dielectric function of graded film can be expressed as

$$
\epsilon(z)=\epsilon_{2}\left(1-\frac{\eta(z)}{s}\right) .
$$

Owing to the simple geometry of a graded film, we can use the equivalent capacitance of a series combination to calculate the effective dielectric constant as

$$
\frac{1}{\epsilon_{e}}=\frac{1}{L} \int_{0}^{L} \frac{1}{\epsilon(z)} \mathrm{d} z
$$

Substituting Eqs. (172) and (173) into Eq. (174), we obtain

$$
\frac{1}{\epsilon_{e}}=\frac{1-h}{\epsilon_{2}}+\frac{s[\ln (1-(\eta(0) / s))-\ln (1-(\eta(h) / s))]}{a \epsilon_{2}},
$$

with the assumption $L=1$. 

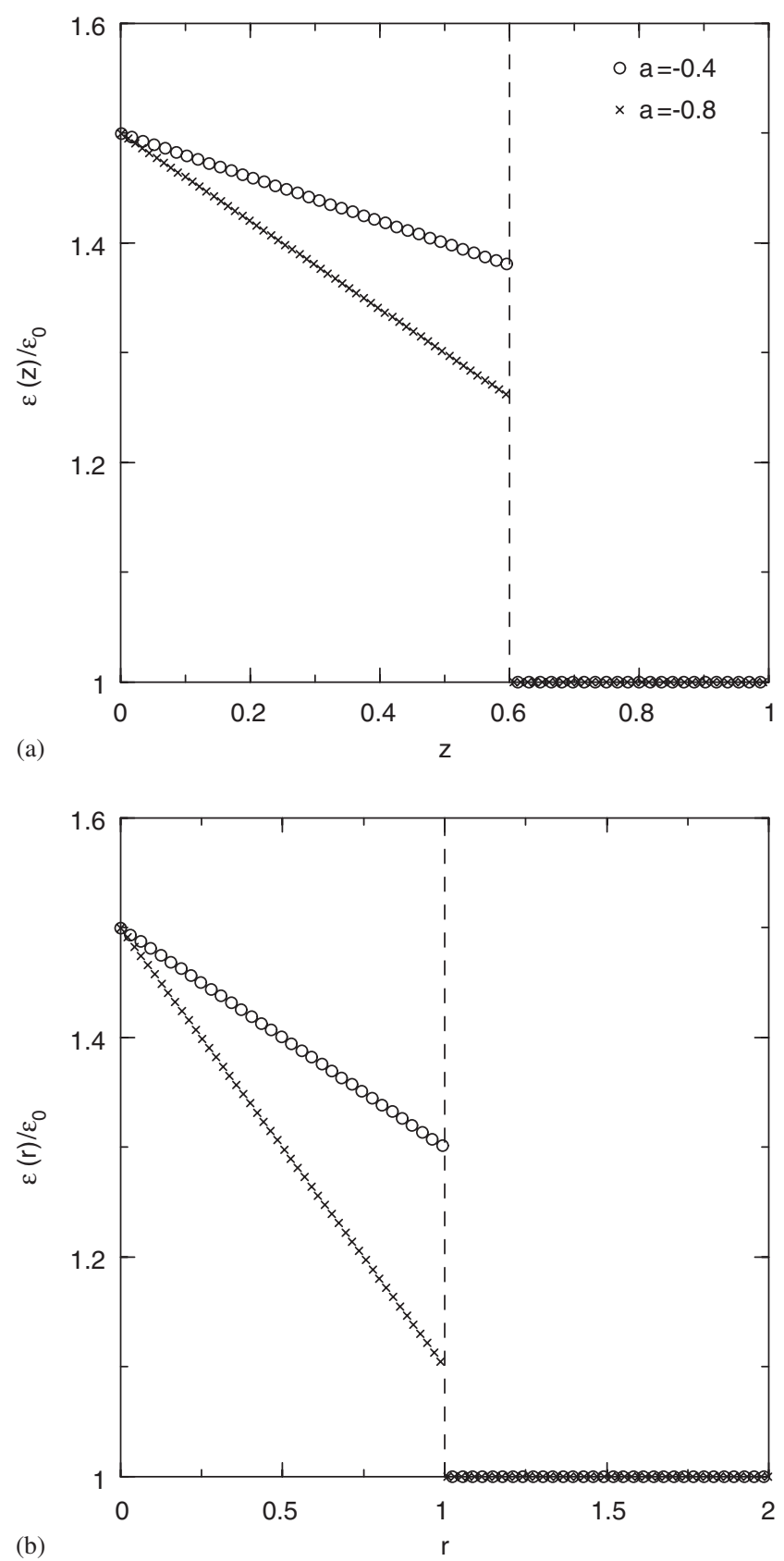

Fig. 44. (a) Dielectric profile of various graded films at $h=0.6$; (b) Dielectric proifle of various graded sphere with unit radius [70]. Parameters used: $\epsilon_{2}=1$ and $s=-2$.

We are now in a position to extend the Bergman-Milton spectral representation of the effective dielectric constant $[3,126,127]$ to a graded film. For a graded system, $\eta(z)$ can be a continuous function in the inclusion medium. Using Eqs. (169)-(171), we obtain the spectral density function for a graded film as

$$
m\left(s^{\prime}\right)=-\frac{a s^{\prime} \arg ((s-1) /(s-a h-1))}{\pi\left[\left(s^{\prime} \arg ((s-1) /(s-a h-1))\right)^{2}+\left(a(h-1)-s^{\prime} \ln \left(\left(s^{\prime}-1\right) /\left(s^{\prime}-a h-1\right)\right)\right)^{2}\right]},
$$

where $s^{\prime}=\operatorname{Re}[s]$ and $\arg [\cdots]$ denote the arguments of complex functions. 


\subsubsection{Spectral density function of a graded sphere}

The above theory can be generalized to graded composites in three dimensions. We consider a graded sphere with dielectric constant $\epsilon_{1}(r)$ embedded into a homogeneous host medium with dielectric constant $\epsilon_{2}$. The dielectric constant of the graded sphere $\epsilon_{1}(r)$ varies along the radius $r$. We can obtain the effective dielectric constant of a graded sphere using the spectral representation. We consider the graded microstructure as

$$
\eta(r)= \begin{cases}1+a r, & 0<r \leqslant R, \\ 0, & r>R,\end{cases}
$$

where $R$ is the radius of the graded sphere. Thus, from Eq. (161) the dielectric constant in the graded sphere is given by

$$
\epsilon(r)=\epsilon_{2}\left(1-\frac{\eta(r)}{s}\right)
$$

In the dilute limit the effective dielectric constant of a small volume fraction $p$ of graded spheres embedded in a host medium is given by $[36,133]$

$$
\epsilon_{e}=\epsilon_{2}+3 \epsilon_{2} p b
$$

where $b$ is the dipole factor of graded spheres embedded in a host as given in Ref. [19]. Using Eqs. (166) and (177), the reduced response can be obtained as

$$
F(s)=-3 \epsilon_{2} p b
$$

Thus, the spectral density function of a graded sphere can be given through a numerical evaluation of Eq. (171).

\subsubsection{Spectral density function of multilayer composites}

A multilayer composite is a special case of graded composites. The gradation becomes continuous as the number of layers approaches infinity. To investigate the multilayer effect, we shall use a finite difference approximation for the graded profile (Eqs. (173) and (176)) for a finite number of layers. To mimic a multilayer system, we divide the interval $[0,1]$ into $N$ equally spaced sub-intervals, $\left[0, z_{1}\right],\left(z_{1}, z_{2}\right], \ldots,\left(z_{N}-1,1\right]$. Then we adopt the midpoint value of $\epsilon(z)$ for each sub-interval as the dielectric constant of that sublayer. In this way, we calculate the effective dielectric constant, eigenvalues, as well spectral density function for each $N$. It is worth noting that the results of $N \rightarrow \infty$ (e.g., $N=1024$ recovers the results of graded composites).

In addition to multilayer films, we can use the above approach to study the much simpler problem of a two-layer film. In this system, we have two layers of dielectric constants $\epsilon_{1}, \epsilon_{2}$, and host $\epsilon_{0}$. Thickness are $h y, h(1-y)$, and $1-h$, respectively, where $y$ is the length ratio between component $\epsilon_{1}$ and component $\epsilon_{2}$. We also define two microstructure parameters, $\eta_{1}$ and $\eta_{2}$. If we let $s=1 /\left(1-\epsilon_{1} / \epsilon_{0}\right)$, then $\eta_{1}=1$, and $\eta_{2}=\left(\epsilon_{0}-\epsilon_{2}\right) /\left(\epsilon_{0}-\epsilon_{1}\right)$. According to Eq. (173), the effective dielectric constant of the two-layer film is now given by

$$
\frac{1}{\epsilon_{e}}=\frac{h y}{\epsilon_{1}}+\frac{h(1-y)}{\epsilon_{2}}+\frac{1-h}{\epsilon_{0}}
$$

According to Eq. (166), the reduced response can be given by

$$
F(s)=\frac{F_{1}}{s-s_{1}}+\frac{F_{2}}{s-s_{2}},
$$


where

$$
\begin{aligned}
F_{1}= & \frac{h\left(s_{1}(y-y \eta+\eta)-\eta\right)}{s_{1}-s_{2}}, \\
F_{2}= & -\frac{h\left(s_{2}(y-y \eta+\eta)-\eta\right)}{s_{1}-s_{2}}, \\
s_{1}= & \frac{1}{2}[1-h(y-y \eta+\eta)+\eta \\
& \left.-\sqrt{4 \eta(-1+h)+(1-h(y-y \eta+\eta)+\eta)^{2}}\right], \\
s_{2}= & \frac{1}{2}[1-h(y-y \eta+\eta)+\eta \\
& \left.+\sqrt{4 \eta(-1+h)+(1-h(y-y \eta+\eta)+\eta)^{2}}\right] .
\end{aligned}
$$

From the sums of $F_{1}$ and $F_{2}$ and the integral of graded microstructure $\eta(z)$ given by Eq. (172), we can check that the sum rule expressed by Eq. (168) is obeyed. It should also be noted that there are two poles in the expression for the reduced response corresponding to two peaks in the spectral density function. If $h=1$, then $s_{1}=0$, that is, one peak is located at zero, which is explicitly shown in Fig. 45(a).

Similarly, we can also apply our graded spectral representation to a single-shell sphere of core dielectric constant $\epsilon_{1}$, covered by a shell of $\epsilon_{2}$, and suspended in a host of $\epsilon_{0}$. In this example, we can also define two microstructure parameters $\eta_{1}$ and $\eta_{2}$. If we let $s=1 /\left(1-\epsilon_{1} / \epsilon_{0}\right)$, then $\eta_{1}=1$, and $\eta_{2}=\left(\epsilon_{0}-\epsilon_{2}\right) /\left(\epsilon_{0}-\epsilon_{1}\right)$. The dipole factor of single-shell sphere is given as $[36,133]$

$$
b=\frac{\epsilon_{2}-\epsilon_{0}+\left(\epsilon_{0}+2 \epsilon_{2}\right) \rho_{1} f^{3}}{\epsilon_{2}+2 \epsilon_{0}+2\left(\epsilon_{2}-\epsilon_{0}\right) \rho_{1} f^{3}},
$$

where $f$ is the ratio between radius core and radius shell, and $\rho_{1}$ is given by

$$
\rho_{1}=\frac{\epsilon_{1}-\epsilon_{2}}{\epsilon_{1}+2 \epsilon_{2}} .
$$

Then, we can also write Eq. (178) similarly to Eq. (179), where the residues and eigenvalues are given by

$$
\begin{aligned}
& F_{1}=\frac{-3 p s_{1}\left[(-1+\eta) y^{3}-\eta\right]-\eta p\left[1-2(-1+\eta) y^{3}+2 \eta\right]}{3\left(s_{1}-s_{2}\right)}, \\
& F_{2}=\frac{3 p s_{2}\left[(-1+\eta) y^{3}-\eta\right]+\eta p\left[1-2(-1+\eta) y^{3}+2 \eta\right]}{3\left(s_{1}-s_{2}\right)}, \\
& s_{1}=\frac{1}{6}\left[1+3 \eta-\sqrt{1+\left(2-8 y^{3}\right) \eta+\left(1+8 y^{3}\right) \eta^{2}}\right], \\
& s_{2}=\frac{1}{6}\left[1+3 \eta+\sqrt{1+\left(2-8 y^{3}\right) \eta+\left(1+8 y^{3}\right) \eta^{2}}\right] .
\end{aligned}
$$

Analysis shows that the spectral representation for $N=2$ contains two simple poles corresponding to two peaks in the spectral density function. Therefore, we draw the conclusion that, $N$ peaks are a result of $N$ layers. Moreover, $N-1$ peaks will accumulate into a continuous broad absorption spectrum when $N$ tends toward infinity, which can be seen from Figs. 47(f) and 48(f).

We are now in a position to do some numerical calculations of the spectral density function from Eqs. (169) and (171). A small but finite imaginary part in the complex parameter has been used in the calculations. Without any loss of generality, we choose $L=1$ and $R=1$ for convenience. We show the effect of different graded profiles, as well 

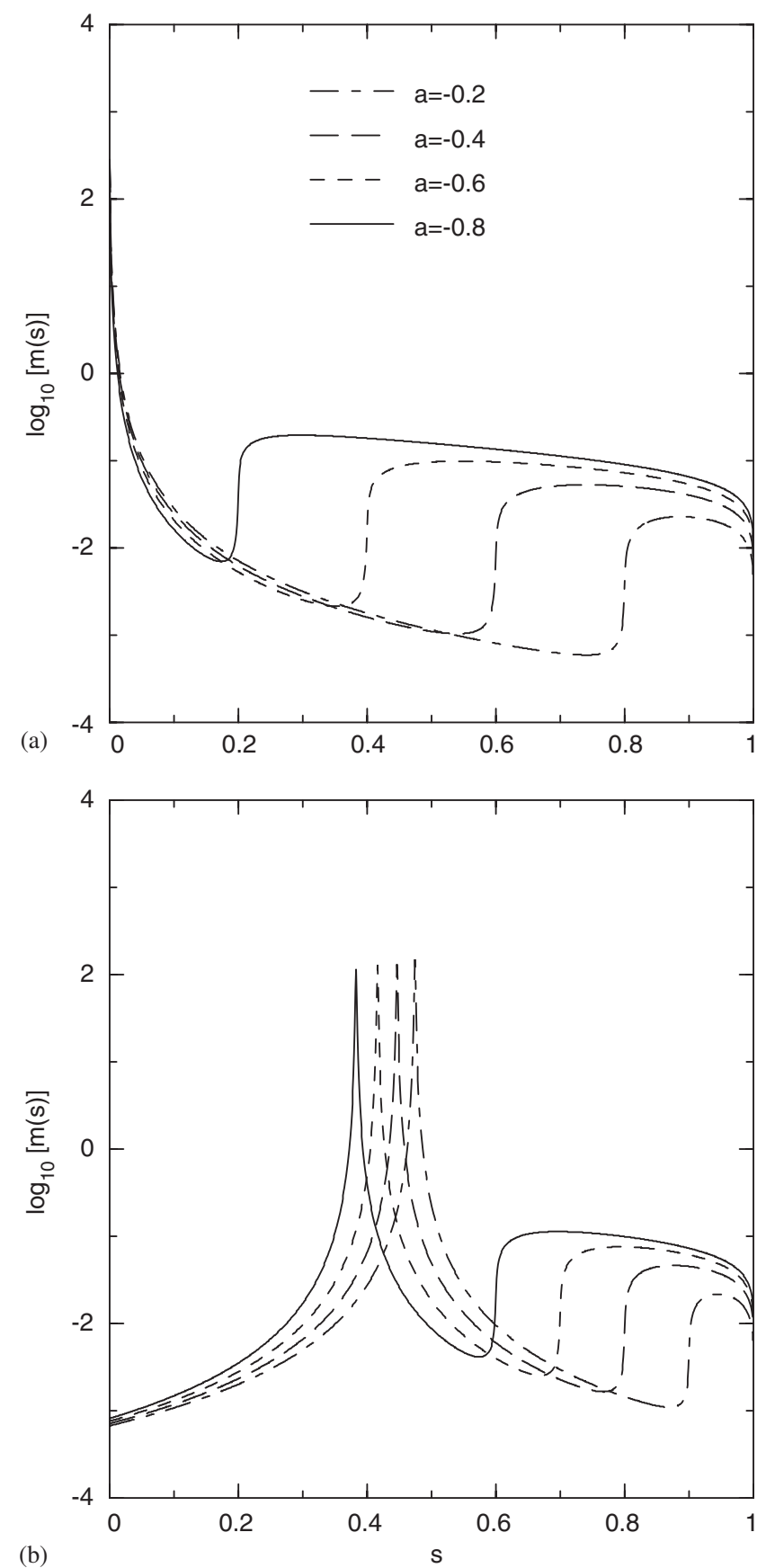

Fig. 45. (a) Spectral density function of a graded film without an interface, i.e., $\mathrm{h}=1.0$; (b) Spectral density function of a graded film meeting a homogeneous medium at an interface $h=0.5$, and $\epsilon_{2}=1[70]$.

as the effect of the thickness of the inclusion. It should be noted, that in all figures the range of $s$ is limited to $[0,1]$, because we chose $-1<a<0$ which limits the value of $\eta$ into $[0,1]$.

Fig. 44 displays the dielectric profile of a graded film (Fig. 44(a)) and a graded sphere (Fig. 44(b)). This figure obviously shows that the dielectric constant varies with the position in inclusion while a constant in host medium. Also, different values of $a$ accord with different graded materials. 


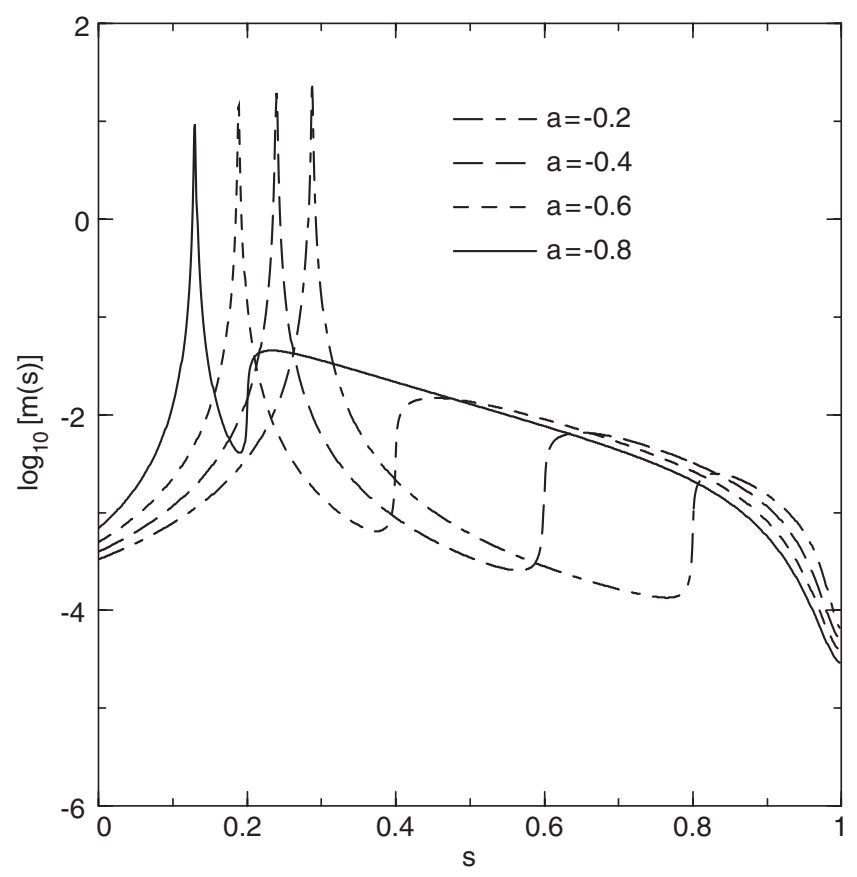

Fig. 46. Spectral density function of a graded sphere with volume fraction $p=0.1[70]$.

In Fig. 45(a), we plot the spectral density function $m(s)$ of a graded film without an interface against the spectral parameter for various graded microstructures $\eta(z)$. It is evident that there is always a broad continuous band in the spectral density function. Both the strength as well as the width of the continuous part of $m(s)$ increase with the gradient of the dielectric profile. Thus, the previous results of the broad surface-plasmon band can be expected. Note that there is a sharp peak at $s=0$, which is also present in a homogeneous film. In Fig. 45(b), we plot the spectral density function of a graded film meeting a homogeneous medium at an interface for various graded microstructure $\eta(z)$. Again, there is always a broad continuous band in the spectral density function. However, the sharp peak has now shifted to a finite value of $s$, which is also present in a homogeneous film.

In Fig. 46, the spectral density function of graded sphere is displayed for a volume fraction $p=0.1$. In this case, the interface always exists. It is clear that a broad continuous function in the spectral density function is always observed, as well as the shift of the sharp peak. However, the decrease of the broad continuous function is more abrupt for graded sphere than for graded film with increasing $s$.

Figs. 47 and 48 display the spectral density function for a multilayer film and a sphere, respectively. It is clear that there are always $N$ sharp peaks for $N$ layers. Moreover, it is worth noting that there occurs a transition from sharp peaks to a broad continuous band with increasing $N$ (see Figs. 47(f) and 48(f)), that is, the graded results are recovered by the limit results of $N \rightarrow \infty$. In particular, we had obtained the analytical expression of spectral density function for $N=2$. There are two resonances corresponding to the two peaks in Figs. 47(a) and 48(a).

We have investigated a graded composite film and a sphere by means of the Bergman-Milton spectral representation. It has been shown that the spectral density function can be obtained analytically for a graded system. However, unlike in the case of homogeneous constituent components, the characteristic function is a continuous function due to the presence of gradation. Moreover, the derivation as well as some salient properties, namely, the sum rule, the definition of inner product, the definition of the integral-differential operators, and the range of spectral parameters, do change because of the continuous variation of the dielectric profile within the constituent components. It should be noted that in graded composite, the eigenvalues are not limited to $[0,1]$, and they can be extended to $-\infty \leqslant s_{n} \leqslant \infty$ for the full region $\eta$, i.e., $-\infty \leqslant \eta \leqslant \infty$. However, for simplicity, we investigated the spectral density function in $0 \leqslant s \leqslant 1$ by choosing $-1<a<0$ to limit the value of $\eta$ into $[0,1]$.

We also study multilayer composites and calculated the spectral density function versus the number of layers, to explicitly demonstrate that the broad continuous spectrum arises from the accumulation of poles when the number 

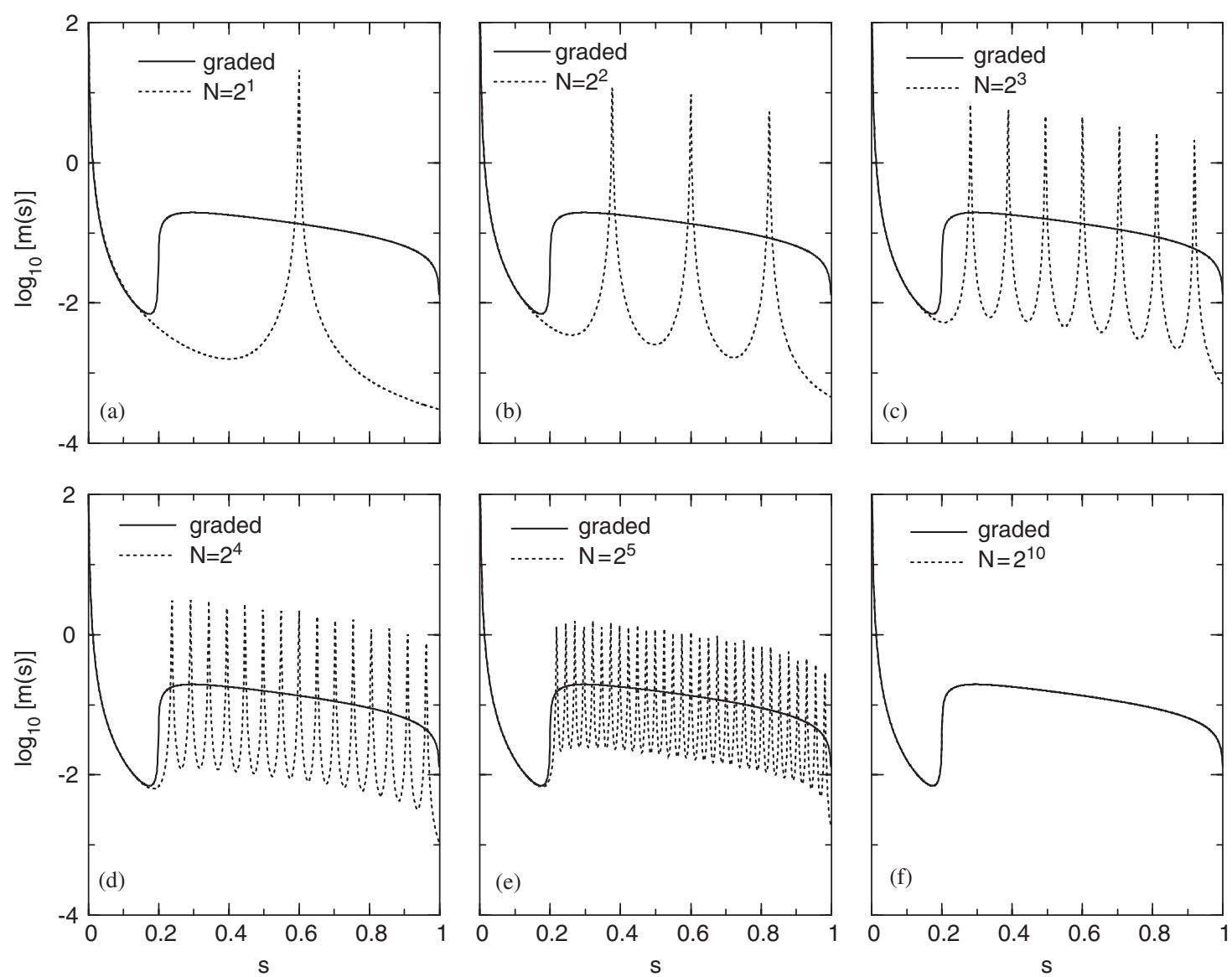

Fig. 47. Spectral density function of various multilayer film with $\epsilon_{0}=1$ and $a=-0.8$ [70].

of layers tends to infinity. This finding coincides with the broad surface-plasmon absorption band associated with the optical properties of graded composites.

To sum up, we have investigated the spectral density function of graded film and graded sphere, as well as multilayer cases. There is always a broad continuous function in the spectral density function in graded composite, but simple poles in multilayer composite, the number of pole depends on the number of layers. Moreover, there is a gradual transition from sharp peaks to a broad continuous band until the graded composite results recover in the limit of $N \rightarrow \infty$.

\section{Magneto-controlled nonlinear optical materials}

The most common way to obtain a nonlinear optical material is to search for materials in which the components possess an inherently large nonlinear response [2]. In contrast, in this section we shall exploit theoretically a nonlinear optical material whose nonlinear optical properties and nonlinearity enhancement can be tuned by applying an external magnetic field - thus called magneto-controlled nonlinear optical materials. Devices that could benefit from these materials include optical switches, optical limiters, etc. Ferromagnetic nanoparticles, typically consisting of magnetite or cobalt, have a typical diameter of $10 \mathrm{~nm}$, and carry a permanent magnetic moment (e.g., of the strength $\sim 2.4 \times 10^{4} \mu_{\mathrm{B}}$ for magnetite nanoparticles, where $\mu_{\mathrm{B}}$ denotes the Bohr magneton) [134]. As the ferromagnetic nanoparticles are suspended in a host fluid like water, they can easily form particle chains under the application of external magnetic fields [134-136], thus yielding a magnetic-field-induced anisotropical structure. Recently, a nonmagnetic golden shell was used to enhance the stability of the ferromagnetic nanoparticle against air and moisture [137]. Below we shall show that the effective nonlinear optical response of the suspension which contains ferromagnetic nanoparticles with 

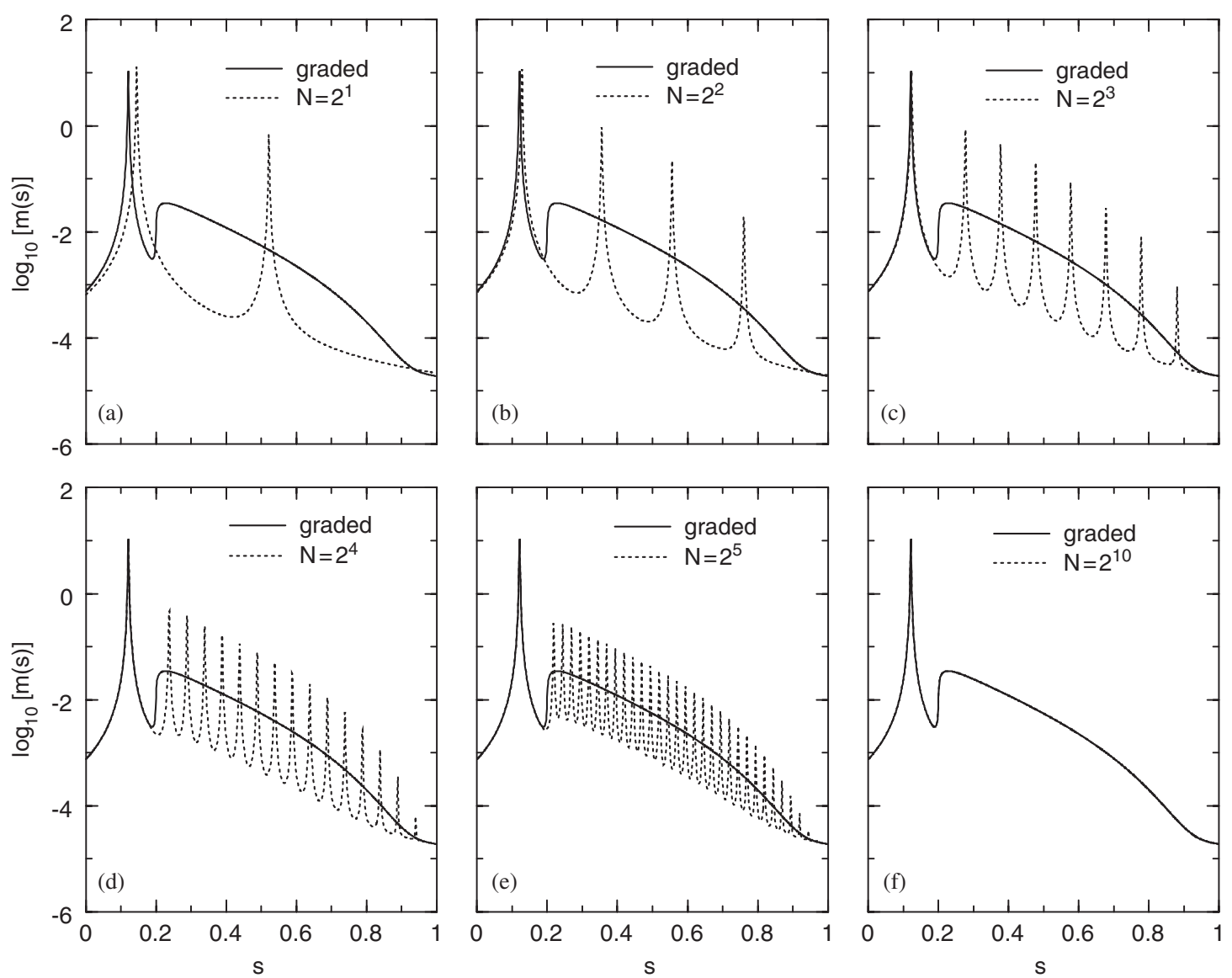

Fig. 48. Spectral density function of various multi-shell sphere with $\epsilon_{0}=1, a=-0.8$ and $p=0.1[70]$.

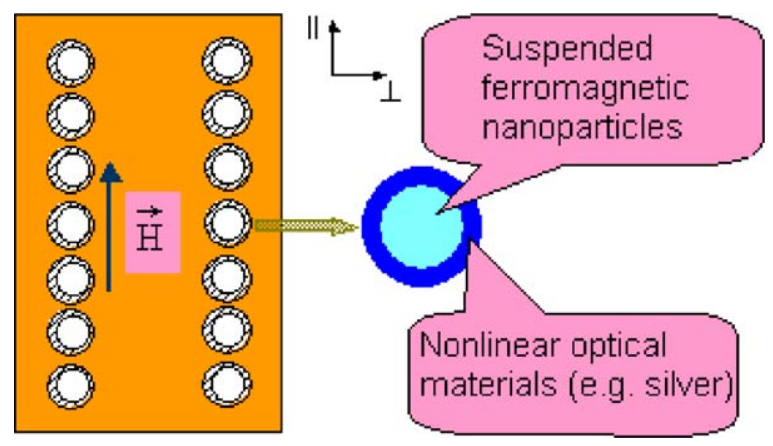

Fig. 49. (Color online) Schematic graph showing the design of magneto-controlled ferrofluid-based nonlinear optical materials [138].

metallic nonlinear shells (see Fig. 49) can be enhanced significantly due to the effect of the magnetic-field-induced anisotropy. For the research on nonlinear optical responses, the introduction of a controllable element (e.g., external magnetic field) should be expected to open a fascinating field of new phenomena.

The third-order nonlinear susceptibility $\chi_{s}$ of metallic (say typically, noble metals like gold and silver) shells is very large when compared to that of the magnetite or cobalt core and the host fluid like water. Let us start by considering ferromagnetic linear nanoparticles of linear dielectric constant $\epsilon_{1}^{\prime \prime}$ coated with a nonmagnetic metallic nonlinear shell 
of $\epsilon_{1}^{\prime}$ and $\chi_{s}$ which are suspended in a linear host fluid of $\epsilon_{2}$. That is, in the shells, there is a nonlinear relation between the displacement $\mathbf{D}_{s}$ and the electric field $\mathbf{E}_{s}, \mathbf{D}_{s}=\epsilon_{1}^{\prime} \mathbf{E}_{s}+\chi_{s}\left|\mathbf{E}_{s}\right|^{2} \mathbf{E}_{s}$, where $\epsilon_{1}^{\prime}$ is given by the Drude form, $\epsilon_{1}^{\prime}=1-\omega_{p}^{2} /[\omega(\omega+\gamma i)]$, where $\omega_{p}$ and $\gamma$ stand for the plasmon frequency and the relaxation rate, respectively, and $\omega$ denotes the frequency of the incident light. In what follows, the thickness of the shell and the radius of the core are respectively denoted as $d$ and $R$. Next, we restrict our discussion to the quasi-static approximation, under which the structured particle or the whole suspension can be regarded as an effective homogeneous one. It is known that the effective third-order nonlinear susceptibility $\bar{\chi}$ of an area [Note the area represents the structured particle and the whole suspension, respectively, see Eqs. (196) and (199) below] is defined as [36,74]

$$
\bar{\chi}=\frac{1}{V\left|E_{0}\right|^{2} E_{0}^{2}} \int_{V} \chi(\mathbf{r})\left|\nabla \phi_{0}(\mathbf{r})\right|^{2}\left[\nabla \phi_{0}(\mathbf{r})\right]^{2} \mathrm{~d} \mathbf{r},
$$

which is in terms of zeroth-order potential $\phi_{0}(\mathbf{r})$ only, see Eqs. (192)-(194) below. In Eq. (190) $E_{0}$ denotes the external applied electric field, $V$ the volume of the area under consideration, $\mathbf{r}$ the local position inside the medium $(r$ the distance from the particle center to the point of interest), and $\chi(\mathbf{r})$ an $\mathbf{r}$-dependent third-order nonlinear susceptibility. To obtain the effective nonlinear susceptibility of the structured particle which contains a linear core with a nonlinear shell, we should obtain the zeroth-order potentials which are actually obtained for the system in which the nonlinear characteristic of shells disappears, $\chi_{s}=0$. Under the quasi-static approximation, the Maxwell equations read

$$
\nabla \times \mathbf{E}=0 \text { and } \nabla \cdot \mathbf{D}=0,
$$

and hence $\mathbf{E}=-\nabla \phi$, where $\phi$ is an electric potential. Solving Eqs. (191) [or the corresponding Laplace equation $\left.\nabla^{2} \phi=0\right]$, we obtain the zeroth-order potentials for the core $\phi_{0}^{c}$, the shell $\phi_{0}^{s}$, and the host $\phi_{0}^{h}$

$$
\begin{aligned}
& \phi_{0}^{c}=-c_{1} E_{0} r \cos \theta, \quad r<R, \\
& \phi_{0}^{s}=-E_{0}\left(c_{2} r-c_{3} r^{-2}\right) \cos \theta, \quad R<r<R+d, \\
& \phi_{0}^{h}=-E_{0}\left(r-c_{4} r^{-2}\right) \cos \theta, \quad r>R+d,
\end{aligned}
$$

where $\theta$ is the angle between the external field and the line joining the particle center and the point under investigation, and the coefficients $c_{1}, c_{2}, c_{3}$, and $c_{4}$ are determined by the appropriate boundary conditions. Owing to Eq. (190), the effective third-order nonlinear susceptibility of the structured particle $\chi_{1}$ can be given by

$$
\chi_{1} \frac{\left\langle\left|\nabla \phi_{0}(\mathbf{r})\right|^{2}\left[\nabla \phi_{0}(\mathbf{r})\right]^{2}\right\rangle_{r \leqslant R+d}}{\left|\mathbf{E}_{0}\right|^{2} \mathbf{E}_{0}^{2}}=f \chi_{s} \frac{\left\langle\left|\nabla \phi_{0}(\mathbf{r})\right|^{2}\left[\nabla \phi_{0}(\mathbf{r})\right]^{2}\right\rangle_{R<r \leqslant R+d}}{\left|\mathbf{E}_{0}\right|^{2} \mathbf{E}_{0}^{2}},
$$

where $f$ is the volume ratio of the shell to the core. Thus, we obtain

$$
\chi_{1}=\chi_{s} \frac{\beta}{\beta^{\prime}},
$$

where $\beta=(3 / 5)\left[1 /(1-f)^{1 / 3}-1\right]|z|^{2} z^{2}\left(5+18 x^{2}+18|x|^{2}+4 x^{3}+12 x|x|^{2}+24|x|^{2} x^{2}\right)$ and

$$
\beta^{\prime}=\left|\frac{\epsilon_{2}}{\epsilon_{2}+(\alpha / 3)\left(\epsilon_{1}-\epsilon_{2}\right)}\right|^{2}\left(\frac{\epsilon_{2}}{\epsilon_{2}+(\alpha / 3)\left(\epsilon_{1}-\epsilon_{2}\right)}\right)^{2}
$$

with $x=\left(\epsilon_{1}^{\prime \prime}-\epsilon_{1}^{\prime}\right) /\left(\epsilon_{1}^{\prime \prime}+2 \epsilon_{1}^{\prime}\right)$ and $z=(1 / 3)\left[\epsilon_{2}\left(\epsilon_{1}^{\prime \prime}+2 \epsilon_{1}^{\prime}\right)\right] /\left\{\epsilon_{1}^{\prime}\left[\epsilon_{2}+(\alpha / 3)\left(\epsilon_{1}^{\prime \prime}-\epsilon_{2}\right)\right]\right\}$. In Eq. (197), the effective linear dielectric constant $\epsilon_{1}$ of each structured particle can be determined by the well-known asymmetrical Maxwell-Garnett approximation

$$
\frac{\epsilon_{1}-\epsilon_{1}^{\prime}}{\epsilon_{1}+2 \epsilon_{1}^{\prime}}=(1-f) \frac{\epsilon_{1}^{\prime \prime}-\epsilon_{1}^{\prime}}{\epsilon_{1}^{\prime \prime}+2 \epsilon_{1}^{\prime}}
$$

It is worth noting that for the above derivation a local field factor $\alpha$ has already been added, see Eq. (197). As a matter of fact, $\alpha$ should be 1 for the direct evaluation of Eqs. (190)-(194). The aim of the intended introduction of $\alpha$ into Eq. (197) is to include the field-induced anisotropy in the system, at least qualitatively. In detail, $\alpha$ denotes the local 
field factors $\alpha_{\mathrm{L}}$ and $\alpha_{\mathrm{T}}$ for longitudinal and transverse field cases, respectively. Here the longitudinal (or transverse) field case corresponds to the fact that the $E$-field of the light is parallel (or perpendicular) to the particle chain. Similar factors in electrorheological fluids were measured by using computer simulations [139,140], and obtained theoretically [52,53] according to the Ewald-Kornfeld formulation.There is a sum rule for $\alpha_{L}$ and $\alpha_{T}, \alpha_{L}+2 \alpha_{T}=3$ [55]. The parameter $\alpha$ measures the degree of anisotropy, which is induced by the applied magnetic field $H$. More precisely, the degree of the field-induced anisotropy is measured by how much $\alpha$ deviates from unity, $1<\alpha_{\mathrm{T}}<3$ for transverse field cases and $0<\alpha_{\mathrm{L}}<1$ for longitudinal field cases. As $H$ increases $\alpha_{\mathrm{T}}$ and $\alpha_{\mathrm{L}}$ should tend to 3 and 0 , respectively, which is indicative of the formation of more and more particle chains as evident in experiments [134]. So, a crude estimate of $\alpha$ can be obtained from the contribution of chains [141], namely, $\alpha=\left[4 \pi(d+R)^{3} / p\right] \sum_{n=1}^{\infty} n \gamma_{n}(H) g_{n}$, where $p$ denotes the volume fraction of the structured particles in the suspension, $g_{n}$ the depolarization factor for a chain with $n$ structured particles, and $\gamma_{n}(H)$ the density of the chain which is a function of $H$. It is noteworthy that for given $p, \gamma_{n}(H)$ also depends on the dipolar coupling constant which relates the dipole-dipole interaction energy of two contacting particles to the thermal energy. Now, the system of interest can be equivalent to the one in which all the particles with linear dielectric constant $\epsilon_{1}$ [Eq. (198)] and nonlinear susceptibility $\chi_{1}$ [Eq. (196)] are embedded in a host fluid with $\epsilon_{2}$. For the equivalent system, it is easy to solve the corresponding Maxwell equations [Eqs. (191)], in order to get the zeroth-order potentials in the particles and the host.According to Eq. (190), we obtain the effective third-order nonlinear susceptibility of the whole suspension $\chi_{e}$ as $\chi_{e}=p \chi_{1} \beta^{\prime}$, which can be rewritten as

$$
\chi_{e}=p \chi_{s} \beta,
$$

The substitution of $\alpha=1.0$ (i.e., the isotropic limit) into Eq. (199) yields the same expression as derived in Ref. [36] in which the dielectric constants of the core and shell of structured particles were, however, assumed to be real rather than complex. On the other hand, the effective linear dielectric constant of the whole suspension under present consideration $\epsilon_{e}$ can be given by the developed Maxwell-Garnett approximation which works for suspensions with field-induced anisotropic structures [52]

$$
\frac{\epsilon_{e}-\epsilon_{2}}{\alpha \epsilon_{e}+(3-\alpha) \epsilon_{2}}=p \frac{\epsilon_{1}-\epsilon_{2}}{\epsilon_{1}+2 \epsilon_{2}}
$$

For numerical calculations, we take $f=0.65, p=0.2, \epsilon_{1}^{\prime \prime}=-25+4 i, \epsilon_{2}=1.77$ (dielectric constant of water), and $\gamma=0.01 \omega_{p}$. We further see $\chi_{s}$ to be a real and positive frequency-independent constant, in order to focus on the nonlinearity enhancement. Figs. 50 and 51 display the linear optical absorption $\operatorname{Im}\left(\epsilon_{e}\right)$, the enhancement of the third-order optical nonlinearity $\left|\chi_{e}\right| / \chi_{s}$, and the figure of merit (FOM) $\left|\chi_{e}\right| /\left[\chi_{s} \operatorname{Im}\left(\epsilon_{e}\right)\right]$ (see Section 1), as a function of normalized frequency $\omega / \omega_{p}$, for (Fig. 50) longitudinal and (Fig. 51) transverse field cases. Here the frequency $\omega$ is normalized by $\omega_{p}$ (rather than a specific value of $\omega_{p}$ ), so that the result could be valid for general cases. As mentioned before, $\alpha=1.0$ corresponds to the isotropic limit. In this case, there is no external magnetic field, and hence all the structured particles are randomly suspended. The figures show that the existence of nonlinear shells causes an enhancement of nonlinearity to appear, see Figs. 50(b) and 51(b), thus yielding a large FOM, see Fig. 50(c) and Fig. 51(c). Such a nonlinearity enhancement induced by shell effects was already reported [36]. The main feature of Figs. 50 and 51 is the effects of external magnetic fields. As $\alpha_{\mathrm{L}}$ changes from 1.0, to 0.6, and to 0.2, (namely, as $\alpha_{\mathrm{T}}$ varies from 1.0, to 1.2, and to 1.4) the external magnetic field is adjusted from zero, to low strength, and to high strength. Due to the interaction between the ferromagnetic nanoparticles and the magnetic field, more and more particle chains are caused to appear naturally, thus yielding a magnetic-field-induced anisotropic structure in the suspension. It is evident to observe that the plasmon peak is caused to be blue-shifted for longitudinal field cases as the magnetic field increases. However, for transverse field cases, the plasmon peak displays a red-shift for the increasing magnetic field. In other words, the optical absorption is induced to be anisotropic due to the application of the external magnetic field which produces an anisotropic structure. In fact, the optical absorption arises from the surface plasmon resonance, which is obtained from the imaginary part of the effective dielectric constant. For single metallic particles in the dilute limit, it is well known that there is a large absorption when the resonant condition $\epsilon_{1}^{\prime}+2 \epsilon_{2}=0$ is fulfilled. When there is a larger volume fraction $p$ of structured particles and an anisotropy $\alpha$ of the suspension, the effective dielectric constant should be obtained from Eq. (200), thus yielding a modified resonant condition $(1-p \alpha) \epsilon_{1}+(2+p \alpha) \epsilon_{2}=0$. So, the resonant frequency becomes larger (smaller) than the isotropic limit $(\alpha=1)$ when $\alpha$ becomes smaller (larger) than 1. In other words, there is a blue (red) shift for the longitudinal (transversal) field cases. More interestingly, for longitudinal field cases, a giant enhancement of nonlinearity is shown as the magnetic field increases, see Fig. 50(b). 

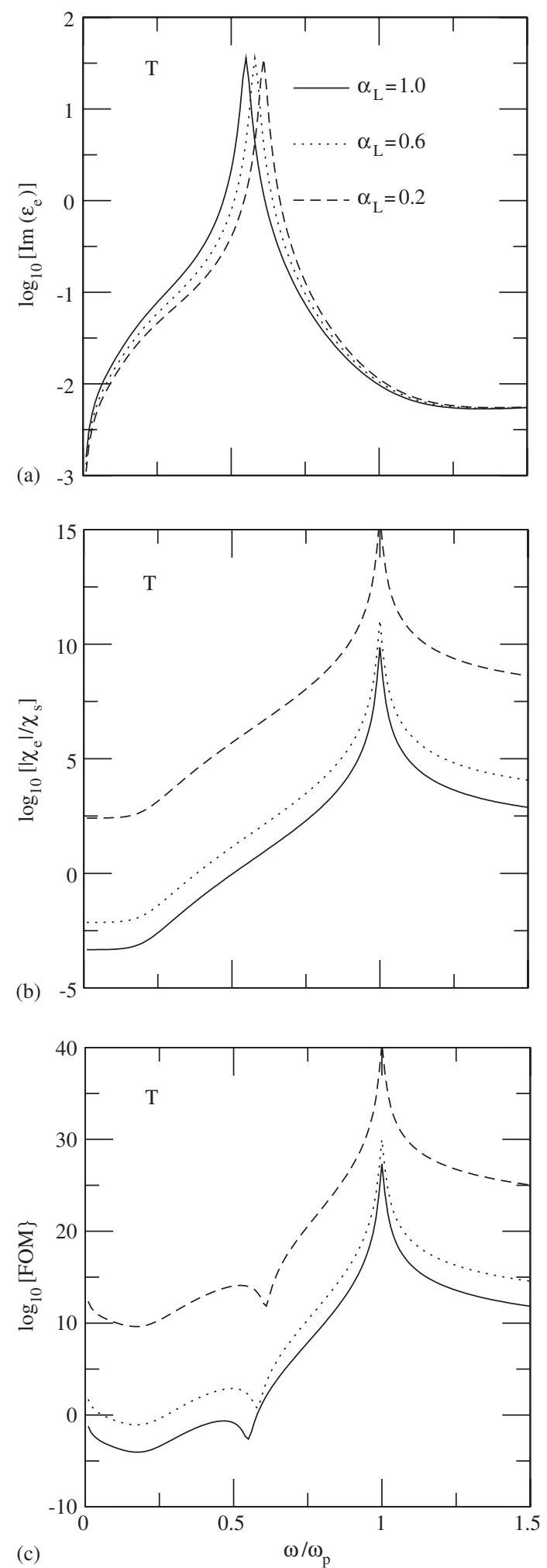

Fig. 50. (a) The linear optical absorption $\operatorname{Im}\left(\epsilon_{e}\right)$, (b) the enhancement of the third-order optical nonlinearity $\left|\chi_{e}\right| / \chi_{s}$, and (c) the FOM $\left|\chi_{e}\right| /\left[\chi_{s} \operatorname{Im}\left(\epsilon_{e}\right)\right]$ versus the normalized incident angular frequency $\omega / \omega_{p}$, for various strengths of the external magnetic field which are represented by local-field factors $\alpha_{\mathrm{L}}$, for longitudinal field cases $(L)$ [9]. 

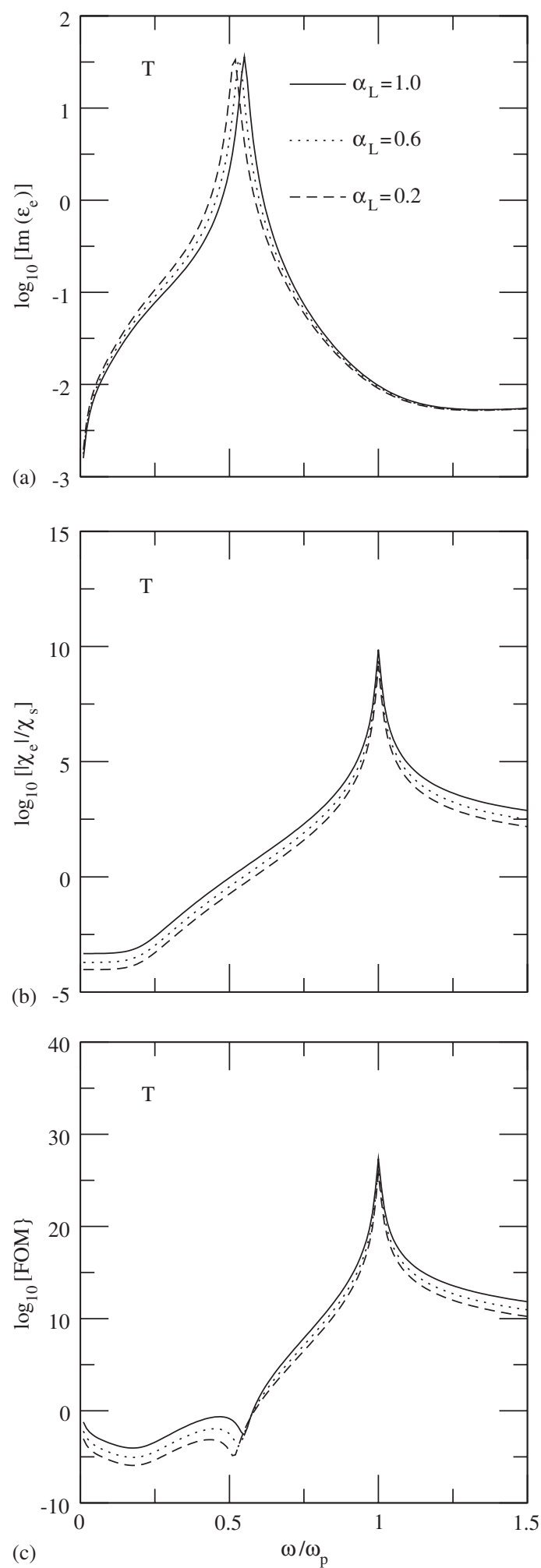

Fig. 51. Same as Fig. 50, but for transverse field cases ( $T$ ) [9]. 
In detail, the nonlinearity enhancement of a high-field case (say, $\alpha=0.2$ ) can be of five orders of magnitude larger than that of the zero-field case $(\alpha=1.0)$. Inversely, a reduction of nonlinearity is found for transverse field cases, see Fig. 51(b). The magnitude of the nonlinearity reduction is very small in the transverse field case, when compared to that of the nonlinearity enhancement in the longitudinal field case. Owing to the giant enhancement of nonlinearity [see Fig. 50(b)], the FOM becomes much more attractive for longitudinal field cases [see Fig. 50(c)]. The FOM of a high-field case (say, $\alpha=0.2$ ) can even be ten-order-of-magnitude enhanced in the longitudinal field case. However, the effect of the magnetic field on the FOM for transverse field cases seems to be uninteresting since the FOM is caused to be decreased slightly due to the nonlinearity reduction shown in Fig. 51(b). Since the permanent magnetic moment of the magnetite nanoparticles $m$ is approximately $2.4 \times 10^{4} \mu_{\mathrm{B}}$ [134], we can estimate the threshold magnetic field $H_{c}=14.3 \mathrm{kA} / \mathrm{m}$ (or threshold magnetic induction $B_{c}=0.018 \mathrm{~T}$ ) above which the corresponding magnetic energy can overcome the thermal energy $1 / 40 \mathrm{eV}$ so as to obtain appreciable anisotropy. Besides the magnetic energy, we should also compare the interaction energy. For instance, for two touching magnetite nanoparticles, the interaction between them is proportional to $m^{2} /[2(d+R)]^{3}$, assuming the two structured particles to be in a head-to-tail alignment. Since the magnetic moment $m$ goes as $(2 R)^{3}$, the interaction energy could vary as $\left[2 R^{2} /(d+R)\right]^{3}$. In order to break up the two touching nanoparticles, the thermal energy should be larger than the interaction energy. So, threshold field $H_{c}=14.3 \mathrm{kA} / \mathrm{m}$ serves as an upper estimate. Nevertheless, for cobalt nanoparticles, the threshold field $H_{c}$ should be lower due to larger permanent magnetic moments.

To sum up, by including a metallic nonlinear shell in the system, one can tune the nonlinear optical properties by applying a magnetic field. Such a proposed magneto-controlled nonlinear optical material can serve as optical materials which have anisotropic nonlinear optical properties and a giant enhancement of nonlinearity, as well as an attractive FOM.

\section{Summary}

Throughout the article, theoretical analysis has been conducted. In looking for experimental support, we find that the separation between absorption peak and nonlinearity enhancement peak proposed by one of us [90] has recently been confirmed experimentally by Guan et al. [142]. In the same article by Guan et al. [142], they also experimentally confirmed our theoretical prediction [54] that the peak of third order nonlinear susceptibilities shifts to longer wavelengths with an increasing applied electric field (which, during fabrication, causes metallic particles to have the form of prolate spheroid).

Composite effects are always expected to open a fascinating field of new phenomena in nonlinear optics. Owing to composite effects, two or more materials can be combined in such a manner that the effective nonlinear optical susceptibility of the composite exceeds those of the constituent materials, which is thus called enhanced nonlinear optical responses. We have investigated the physical processes of the composite effects on the enhanced third-order nonlinear optical responses of the recently-proposed nonlinear optical materials:

1. Colloidal nanocrystals with inhomogeneous metallodielectric particles or a graded-index host

Such materials can have both an enhancement and a red shift of optical nonlinearity, due to the gradation inside the metallic core or host as well as the lattice effects arising from the periodic structure.

\section{Metallic films with inhomogeneous microstructures adjusted by ion doping or temperature gradient}

It has been found that the presence of gradation (or multilayer) in metallic films yields a broad resonant plasmon band in the optical region, resulting in a large nonlinearity enhancement and hence an optimal figure of merit.

3. Composites with compositional gradation or graded particles

We found enhanced nonlinear optical responses can be achieved due to the presence of gradation inside compositionally graded metal-dielectric films and/or dielectrically-graded particles with/without dielectric anisotropy. A spectral representation was developed to understand the enhanced responses.

\section{Magneto-controlled ferrofluid-based nonlinear optical materials}

By including a metallic nonlinear shell in field-responsive ferrofluids, one can tune the enhanced nonlinear optical properties by applying an external magnetic field. Such a proposed magneto-controlled nonlinear optical material can serve as optical materials which have anisotropic nonlinear optical properties and a giant enhancement of nonlinearity, as well as an attractive figure of merit.

The composite effects, which result from strong enhancement or fluctuations of the local fields in the different microstructures, yield enhanced nonlinearity enhancement of the proposed nonlinear optical materials. These materials 
have some features: Firstly, they can offer higher (or anisotropic) effective nonlinear optical responses and hence desired faster response times, due to the many-body (local-field) effect and the long-range lattice effect in the particle chains/columns or clusters in the system subjected to external fields. Secondly, the nonlinear optical responses or the response times can be real-time-adjusted by choosing appropriate external electric or magnetic fields, due to the fieldinduced change of the microstructure in the suspensions. Finally, cost can be saved much since the suspension-based nonlinear optical materials have fluidity and are difficult to be abraded.

Except for enhanced third-order nonlinear optical susceptibilities discussed in the review, there are a number of other optical processes [10,143], e.g., four-wave mixing, second or third harmonic generation, etc. We may also consider composite effects on such nonlinear optical processes, which are also interesting and useful in the sense that they are accessible to experimentalists working in the field. Following the approaches mentioned in the review, new types of nonlinear dielectric materials can also be designed [54,144] for use in electronic and microwave components, sensor windows, and so on.

\section{Acknowledgments}

For completing the review we have profited from valuable and stimulating collaborations and discussions with Prof. Z. Y. Li, Prof. G.Q. Gu, Prof. L. Gao, Dr. M. Karttunen, Prof. K. Yakubo, Prof. T. Nakayama, Prof. C. Holm, Prof. P. M. Hui, Mr. J. J. Xiao, and Ms. L. Dong. J.P.H. would also like to express his gratitude to Ms. C. Z. Fan, Mr. G. Wang, Mr. W. J. Tian, and Ms. Y. J. Zhao, for their helpful assistance. The original research was supported by the Research Grants Council of the Hong Kong SAR Government, by the Alexander von Humboldt Foundation in Germany, by the German Research Foundation under Grant No. HO 1108/8-4, by the Department of Physics, Fudan University, China, and by the Grant-in-Aid for Scientific Research organized by Japan Society for the Promotion of Science. J.P.H. also acknowledges the financial support by the Shanghai Education Committee and the Shanghai Education Development Foundation ("Shu Guang" project) under Grant No. KBH1512203, by the Scientific Research Foundation for the Returned Overseas Chinese Scholars, State Education Ministry, China, by the National Natural Science Foundation of China under Grant No. 10321003, and by Jiangsu Key Laboratory of Thin Films, Suzhou University.

\section{References}

[1] N. Bloembergen, Nonlinear Optics, fourth ed., World Scientific, Singapore, New Jersey, London, Hong Kong, 1996.

[2] D.C. Rodenberger, J.R. Heflin, A.F. Garito, Nature, London 359 (1992) 309.

[3] D.J. Bergman, D. Stroud, Solid State Physics: Applied in Research and Applications, vol. 46, Academic Press, New York, 1992 , pp. 147.

[4] G.L. Fischer, et al., Phys. Rev. Lett. 74 (1995) 1871.

[5] R.S. Bennink, Y.-K. Yoon, R.W. Boyd, J.E. Sipe, Opt. Lett. 24 (1999) 1416.

[6] V.M. Shalaev, Nonlinear Optics of Random Media: Fractal Composites and Metal-Dielectric Films, Springer, Berlin, 2000.

[7] L. Gao, K.W. Yu, Z.Y. Li, B. Hu, Phys. Rev. E 64 (2001) 036615.

[8] T. Sekikawa, A. Kosuge, T. Kanai, S. Watanabe, Nature, London 432 (2004) 605.

[9] J.P. Huang, K.W. Yu, Appl. Phys. Lett. 86 (2005) 041905.

[10] J.P. Huang, K.W. Yu, New Nonlinear Optical Materials: Theoretical Research, Nova science publishers, Inc., New York, in preparation.

[11] J.E. Sipe, R.W. Boyd, Phys. Rev. A 46 (1992) 1614.

[12] I. Tanahashi, Y. Manabe, T. Tohda, S. Sasaki, A. Nakamura, J. Appl. Phys. 79 (1996) 1244.

[13] R.J. Gehr, R.W. Boyd, Chem. Mater. 8 (1996) 1807.

[14] V.M. Shalaev, E.Y. Poliakov, V.A. Markel, Phys. Rev. B 53 (1996) 2437.

[15] V.M. Shalaev (Eds.), Optical Properties of Nanostructured Random Media, Springer, Berlin, 2002.

[16] V.M. Shalaev, Phys. Rep. 272 (1996) 61.

[17] A. Sarychev, V. Shalaev, Phys. Rep. 335 (2000) 275.

[18] N.N. Lepeshkin, A. Schweinsberg, G. Piredda, R.S. Bennink, R.W. Boyd, Phys. Rev. Lett. 93 (2004) 123902.

[19] L. Dong, G.Q. Gu, K.W. Yu, Phys. Rev. B 67 (2003) 224205.

[20] L. Gao, J.P. Huang, K.W. Yu, Phys. Rev. B 69 (2004) 075105.

[21] J.P. Huang, K.W. Yu, Appl. Phys. Lett. 85 (2004) 94.

[22] J.P. Huang, L. Dong, K.W. Yu, Europhys. Lett. 67 (2004) 854.

[23] J.P. Huang, K.W. Yu, Opt. Lett. 30 (2005) 275.

[24] A.M. Freyria, E. Chignier, J. Guidollet, P. Louisot, Biomaterials 12 (1991) 111.

[25] H. Karacali, S.M. Risser, K.F. Ferris, Phys. Rev. E 56 (1997) 4286.

[26] U. Levy, et al., J. Opt. Soc. Am. A 22 (2005) 724.

[27] J.C.M. Garnett, Philos. Trans. R. Soc. London Ser. A 203 (1904) 385.

[28] J.C.M. Garnett, Philos. Trans. R. Soc. London Ser. A 205 (1906) 237. 
[29] D.A.G. Bruggeman, Ann. Phys., Leipzig 24 (1935) 636.

[30] D.J. Bergman, Phys. Rep. 43 (1978) 377.

[31] K.P. Yuen, K.W. Yu, J. Phys.: Condens. Matter 9 (1997) 4669.

[32] W. Wen, H. Ma, W.Y. Tam, P. Sheng, Phys. Rev. E 55 (1997) R1294.

[33] Y. Gu, Q.H. Gong, Phys. Rev. B 67 (2003) 014209.

[34] J.P. Huang, K.W. Yu, J. Phys.: Condens. Matter 14 (2002) 1213.

[35] Z. Hashin, J. Appl. Mech. 29 (1962) 143.

[36] K.W. Yu, P.M. Hui, D. Stroud, Phys. Rev. B 47 (1993) 14150.

[37] K.W. Yu, J.T.K. Wan, M.F. Law, K.K. Leung, Int. J. Mod. Phys. C 9 (1998) 1447.

[38] K. Schulgasser, J. Appl. Phys. 54 (1983) 1380.

[39] G.W. Milton, The Theory of Composites, Cambridge University Press, Cambridge, England, 2002.

[40] K.W. Yu, G.Q. Gu, Phys. Lett. A 345 (2005) 448.

[41] F. Caruso, Colloids and Colloid Assemblies, Wiley-VCH, Weinheim, 2004.

[42] K.P. Velikov, C.G. Christova, R.P.A. Dullens, A.V. Blaaderen, Science 296 (2002) 106.

[43] T. Gong, D.W.M. Marr, Appl. Phys. Lett. 85 (2004) 3760.

[44] T. Schilling, D. Frenkel, Phys. Rev. Lett. 92 (2004) 085505.

[45] B.V.R. Tata, P.S. Mohanty, M.C. Valsakumar, J. Yamanaka, Phys. Rev. Lett. 93 (2004) 268303.

[46] P. Schall, I. Cohen, D.A. Weitz, F. Spaepen, Science 305 (2004) 1944.

[47] J.H. Holtz, S.A. Asher, Nature, London 389 (1997) 829.

[48] Y.A. Vlasov, X.Z. Bo, J.C. Strum, D.J. Norris, Nature, London 414 (2001) 289.

[49] J.P. Huang, K.W. Yu, Appl. Phys. Lett. 87 (2005) 071103.

[50] C.L. Nehl, et al., Nano Lett. 4 (2004) 2355.

[51] D.B. Mitzi, L.L. Kosbar, C.E. Murray, M. Copel, A. Afzali, Nature, London 428 (2004) 299.

[52] C.K. Lo, K.W. Yu, Phys. Rev. E 64 (2001) 031501.

[53] J.P. Huang, Phys. Rev. E 70 (2004) 041403.

[54] J.P. Huang, J. Phys. Chem. B 109 (2005) 4824.

[55] L.D. Landau, E.M. Lifshitz, L.P. Pitaevskii, Electrodynamics of Continuous Media, second ed., Pergamon Press, New York, 1984.

[56] D. Wang, et al., Small 1 (2005) 122.

[57] H. Ma, R. Xiao, P. Sheng, J. Opt. Soc. Am. B 15 (1998) 1022.

[58] A.V. Blaaderen, MRS Bull. 29 (2004) 85.

[59] L. Dong, J.P. Huang, K.W. Yu, G.Q. Gu, J. Appl. Phys. 95 (2004) 621.

[60] L. Gao, J.P. Huang, K.W. Yu, Eur. Phys. J. B 36 (2003) 475.

[61] R.J. Elliot, J.A. Krumhansl, P.L. Leath, Rev. Mod. Phys. 46 (1974) 465.

[62] S. Riikonen, I. Romero, F.J.G. de Abajo, Phys. Rev. B 71 (2005) 235104.

[63] J.J. Xiao, K.W. Yu, Appl. Phys. Lett. 88 (2006) 071911.

[64] J. Lekner, Physica A 157 (1989) 826.

[65] J. Lekner, Physica A 176 (1991) 485.

[66] S.V. Lishchuk, Mol. Phys. 100 (2002) 3789.

[67] G.Q. Gu, K.W. Yu, Phys. Rev. B 46 (1992) 4502.

[68] X.C. Zeng, D.J. Bergman, P.M. Hui, D. Stroud, Phys. Rev. B 38 (1988) 10970.

[69] A. Sharkawy, et al., Opt. Express 13 (2005) 2814.

[70] L. Dong, M. Karttunen, K.W. Yu, Phys. Rev. E 72 (2005) 016613.

[71] D.R. Kammler, et al., J. Appl. Phys. 90 (2001) 5979.

[72] S.G. Lu, et al., Appl. Phys. Lett. 82 (2003) 2877.

[73] M. Yamanouchi, M. Koizumi, T. Hirai, I. Shioda (Eds.), Proceedings of the First International Symposium on Functionally Graded Materials, Sendi, Japan, 1990.

[74] D. Stroud, P.M. Hui, Phys. Rev. B 37 (1988) 8719.

[75] G.S. Agarwal, S.D. Gupta, Phys. Rev. A 38 (1988) 5678.

[76] B.K.P. Scaife, Principles of Dielectrics, Calvendon, Oxford, 1989.

[77] R.W. Boyd, J.E. Sipe, J. Opt. Soc. Am. B 11 (1994) 297.

[78] L. Gao, J.T.K. Wan, K.W. Yu, Z.Y. Li, J. Appl. Phys. 88 (2000) 1893.

[79] D.J. Bergman, Phys. Rev. B 39 (1989) 4598.

[80] A.E. Neeves, M.H. Birnboim, J. Opt. Soc. Am. B 6 (1989) 787.

[81] B. Pettinger, X. Bao, I.C. Wilcock, M. Muhler, G. Ertl, Phys. Rev. Lett. 72 (1994) 1561.

[82] H.-P. Chiang, P.T. Leung, W.S. Tse, J. Phys. Chem. B 104 (2000) 2348.

[83] J.P. Huang, L. Dong, K.W. Yu, J. Appl. Phys. 99 (2006) 053503.

[84] J.W. Haus, R. Inguva, C.M. Bowden, Phys. Rev. A 40 (1989) 5729.

[85] K.P. Yuen, K.W. Yu, J. Opt. Soc. Am. B 14 (1997) 1387.

[86] M. Tlidi, M.F. Hilali, P. Mandel, Europhys. Lett. 55 (2001) 26.

[87] P. Mulvaney, MRS Bull. 26 (2001) 1009.

[88] S. Roorda, et al., Adv. Mater. 16 (2004) 235.

[89] C. Sönnichsen, et al., Phys. Rev. Lett. 88 (2002) 077402. 
[90] K.P. Yuen, M.F. Law, K.W. Yu, P. Sheng, Phys. Rev. E 56 (1997) R1322.

[91] B.M.I.V.D. Zande, L. Pages, R.A.M. Hikmet, A.V. Blaaderen, J. Phys. Chem. B 103 (1999) 5761.

[92] E. Snoeks, et al., Adv. Mater. 12 (2000) 1511.

[93] A. Benyagoub, et al., Nucl. Instr. Methods Phys. Res. B 64 (1992) 684.

[94] B. Xu, J.P. Huang, K.W. Yu, Phys. Lett. A, 2006, in press.

[95] H. Grull, A. Schreyer, N.F. Berk, C.F. Majkrzak, C.C. Han, Europhys. Lett. 50 (2000) 107.

[96] R.S. Bennink, Y.-K. Yoon, R.W. Boyd, J.E. Sipe, Opt. Lett. 24 (1999) 1416.

[97] W.T. Wang, et al., Appl. Phys. Lett. 83 (2003) 1983.

[98] T.B. Jones, Electromechanics of Particles, Cambridge University Press, Cambridge, England, 1995.

[99] J.P. Huang, K.W. Yu, J. Opt. Soc. Am. B 22 (2005) 1640.

[100] D. Stroud, V.E. Wood, J. Opt. Soc. Am. B 6 (1989) 778.

[101] C.F. Bohren, D.R. Huffman, Absorption and Scattering of Light by Small Particles, Wiley, New York, 1983.

[102] Z. Hashin, S. Shtrikman, J. Appl. Phys. 33 (1962) 3125.

[103] K.W. Yu, Solid State Commun. 105 (1998) 689.

[104] H.K. Chan, C.H. Lam, F.G. Shin, J. Appl. Phys. 95 (2004) 2665.

[105] Z.H. Jin, N. Noda, Int. J. Eng. Sci. 31 (1993) 793.

[106] Z.J. Sanchez-Herencia, R. Mereno, J.R. Jurado, J. Euro. Ceram. Soc. 20 (2000) 1611.

[107] F. Erdogan, A.C. Kaya, P.E. Joseph, J. Appl. Mech. 58 (1991) 410.

[108] S. Chandresekhar, Liquid Crystals, second ed., Cambridge University Press, Cambridge, 1992.

[109] J.D. Jackson, Classical Electrodynamics, Wiley, New York, 1975.

[110] G.Q. Gu, K.W. Yu, J. Appl. Phys. 94 (2003) 3376.

[111] J.P. Huang, K.W. Yu, G.Q. Gu, M. Karttunen, Phys. Rev. E 67 (2003) 051405.

[112] K.W. Yu, G.Q. Gu, J.P. Huang, URL http://www.arxiv.org/pdf/cond-mat/0211532.

[113] L. Gao, J.P. Huang, K.W. Yu, Phys. Rev. E 67 (2003) 021910.

[114] L. Gao, Z.Y. Li, J. Appl. Phys. 91 (2002) 2045.

[115] J.P. Huang, L. Gao, K.W. Yu, G.Q. Gu, Phys. Rev. E 69 (2004) 036605.

[116] K.W. Yu, G.Q. Gu, Phys. Lett. A 193 (1994) 311.

[117] K.W. Yu, P.M. Hui, H.C. Lee, Phys. Lett. A 210 (1996) 115.

[118] M. Avellaneda, A. Cherkaev, K. Lurie, G. Milton, J. Appl. Phys. 63 (1988) 4989.

[119] J.H. Erdmann, S. Zumer, J. Doane, Phys. Rev. Lett. 64 (1990) 1907.

[120] V.L. Sukhorukov, G. Meedt, M. Kürschner, U. Zimmermann, J. Electrost. 50 (2001) 191.

[121] D. Stroud, Phys. Rev. B 54 (1996) 3295.

[122] J. Roth, M.J. Dignam, J. Opt. Soc. Am. 63 (1973) 308.

[123] S. Barabash, D. Stroud, J. Phys.: Condens. Matter 11 (1999) 10323.

[124] L. Gao, J.T.K. Wan, K.W. Yu, Z.Y. Li, J. Phys.: Condens. Matter 12 (2000) 6825.

[125] O. Levy, D. Stroud, Phys. Rev. B 56 (1997) 8035.

[126] G.W. Milton, Appl. Phys. A 26 (1981) 1207.

[127] G.W. Milton, J. Appl. Phys. 52 (1980) 5286.

[128] M.P. Hobson, J.E. Baldwin, Appl. Opt. 43 (2004) 2651.

[129] S. Martin, J. Rivory, M. Schoenauer, Appl. Opt. 34 (1995) 2247.

[130] P.G. Verly, Appl. Opt. 37 (1998) 7327.

[131] O. Levy, D.J. Bergman, Physica A 207 (1994) 157.

[132] H. Ma, B. Zhang, W.Y. Tam, P. Sheng, Phys. Rev. B 61 (2000) 962.

[133] X. Zhang, D. Stroud, Phys. Rev. B 49 (1994) 944.

[134] S. Odenbach, Magnetoviscous Effects in Ferrofluids, Springer, Berlin, 2002.

[135] J.P. Huang, J. Phys. Chem. B 108 (2004) 13901.

[136] J.P. Huang, Z.W. Wang, C. Holm, Phys. Rev. E 71 (2005) 061203.

[137] H. Bönnemann, et al., Inorganica Chimica Acta 350 (2003) 617.

[138] C.Z. Fan, J.P. Huang, Appl. Phys. Lett. 2006, submitted for publication.

[139] J.E. Martin, R.A. Anderson, C.P. Tigges, J. Chem. Phys. 108 (1998) 3765.

[140] J.E. Martin, R.A. Anderson, C.P. Tigges, J. Chem. Phys. 108 (1998) 7887.

[141] M. Rasa, J. Magn. Magn. Mater. 201 (1999) 170.

[142] D.Y. Guan, Z.H. Chen, Y.L. Zhou, K.J. Jin, G.Z. Yang, Appl. Phys. Lett. 88 (2006) 111911.

[143] Y.R. Shen, The Principles of Nonlinear Optics, Wiley, New York, 1984.

[144] J.P. Huang, Phys. Rev. E 70 (2004) 042501. 